\title{
COUPLED TWO-DIMENSIONAL EDGE PLASMA AND NEUTRAL GAS MODELING OF TOKAMAK SCRAPE-OFF-LAYERS
}

\author{
by
}

\section{Rajesh Maingi}
A dissertation submitted to the Graduate Faculty of North Carolina State University in partial fulfillment of the requirements for the Degree of Doctor of Philosophy

Department of Nuclear Engineering

Raleigh

August, 1992 


\section{COUPLED TWO-DIMENSIONAL EDGE LASMA AND NEUTRAL GAS MODELING OF TOKAMAK \& SAPE-OFF-LAYERS}

by

\section{Rajesh Maingi}
A dissertation submitted to the Graduate Faculty of North Carolina State University in partial fulfillment of the requirements for the Degree of Doctor of Philosophy

\section{Department of Nuclear Engineering}

Raleigh

August, 1992

APPROVED BY:
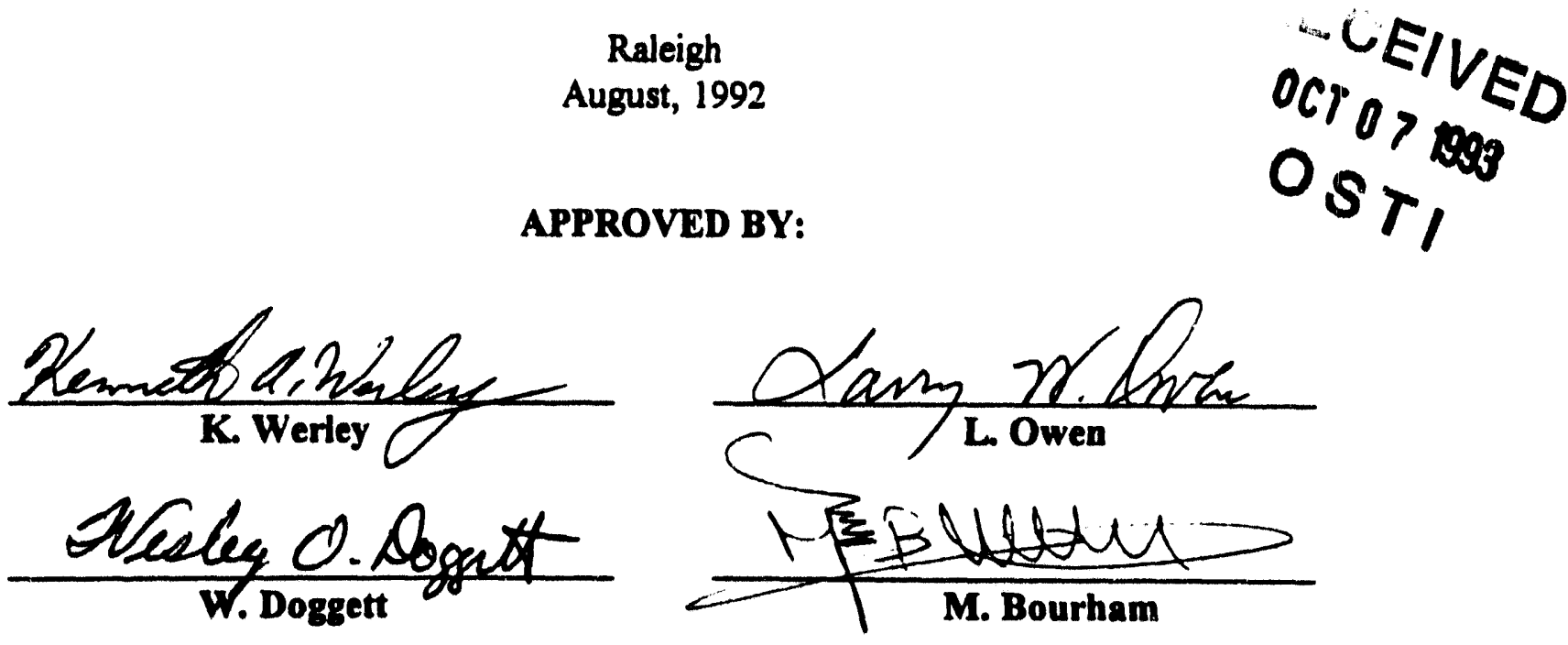

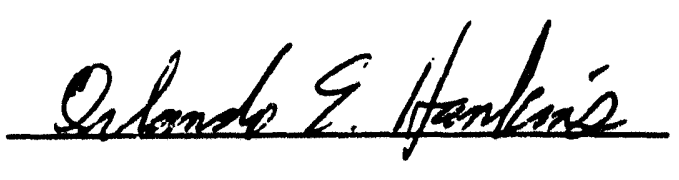

O. Hankins

Co-chair of Advisory Committee

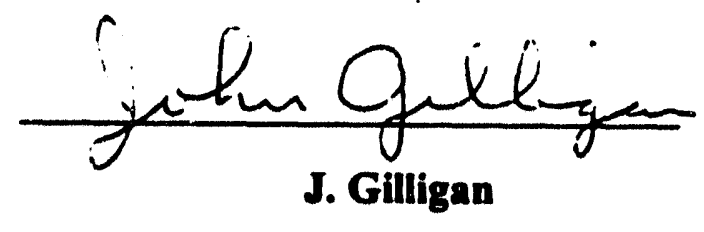

Co-chair of Advisory Committee 


\begin{abstract}
MANNGI, RAJESH. Coupled Two-dimensional Edge Plasma and Neutral Gas Modeling of Tokamak Scrape-off-layers. (Under the direction of John G. Gilligan and Orlando E. Hankins.)

The objective of this study is to devise a detailed description of the tokamak scrape-off-layer (SOL), which includes the best available models of both the plasma and neutral species and the strong coupling between the two in many SOL regimes. A good estimate of both particle flux and heat flux profiles at the limiter/divertor target plates is desired. Peak heat flux is one of the limiting factors in determining the survival probability of plasma-facing-components at high power levels. Plate particle flux affects the neutral flux to the pump, which determines the particle exhaust rate. A technique which couples a two-dimensional (2-D) plasma and a 2-D neutral transport code has been developed (coupled code technique), but this procedure requires large amounts of computer time. Relevant physics has been added to an existing two-neutral-species model which takes the SOL plasma/neutral coupling into account in a simple manner (molecular physics model), and this model is compared with the coupled code technique mentioned above. The molecular physics model is benchmarked against experimental data from a divertor tokamak (DIII-D), and a similar model (single-species model) is benchmarked against data from a pump-limiter tokamak (Tore Supra). The models are then used to examine two key issues: free-streaming-limits (ion energy conduction and momentum flux) and the effects of the non-orthogonal geometry of magnetic flux surfaces and target plates on edge plasma parameter profiles.
\end{abstract}

In general, the calculations by the molecular physics model are in good agreement with the coupled code technique calculations. Calculations by the molecular physics model 
indicate that divertor pumping should not significantly increase the peak heat flux to the target plates. In addition, the use of the kinetic-based free-streaming-limit on ion conduction, i.e. limiting of the ion heat flux based on the ion sound speed, is shown to have a large impact on SOL parameters, confirming previous calculations without the coupled codes. Also, preliminary calculations using a non-orthogonal 2-D grid indicate that the expected heat flux reduction from tilting of the target plates is not as large as computed from extrapolations of orthogonal grid simulations. Further investigation is required to confirm the non-orthogonal grid results. The low plasma density regime existing in the pump-limiter SOL reduces the impact of the recycling sources on plasma parameters, and the calculations from both the single-species model and coupled code technique are in good agre:ment with measurements from an analyzed shot. Omission of the momentum flux free-streaming-limit, i.e. limiting of the ion momentum flux based on the ion sound speed, is shown tc have a large impact on the plasma density and pressure profiles very close to the plate, and the plate peak heat flux. 


\section{DEDICATION}

This thesis is dedicated to all members of my immediate and extended family: to all of my relatives in India, whom I wish could be here now; to my relatives in the United States, who are here to share this honor with me; to my brother and his family for maintaining strong family ties and spurring competition in my younger days; to my parents for teaching me some of the most important values of life, such as perserverance, loyalty, and honesty. But most of all, this thesis is dedicated to my son, Logan Suneil, who is one of the primary sources of my motivation, and my wife Nola, who reminds me to relax and enjoy life once in a while. 


\section{ACKNOWLEDGEMENTS}

There are many people without whom this study may not have been accomplished. First of all, I wish to thank all of the members on my advisory committee for their guidance and advice. In addition, special thanks are given to Ken Weriey, who helped me to understand the basics when I started edge plasma modeling at Los Alamos National Laboratory in 1989. Next, special thanks are given to Larry Owen, Peter Mioduszewski, Chris Klepper, John Hogan, and Taner Uckan of the Edge Plasma and Particle Control Group at Oak Ridge National Laboratory for assisting/guiding me through portions of this analysis. Finally special thanks are given to Marv Rensink for his assistance with the grid-generation scheme and the simulation interpretation. Special thanks are also given to Tom Rognlien who arranged for several visits to Lawrence Livermore National Laboratory. Of course, special thanks are given to the authors of the 2 computer codes I used, Bastiaan Braams and Daniel Heifitz. Thanks are also given to Daren Stotler and David Coster for sharing their valuable experience with the use of the 2 codes.

Finally special thanks are given to the United Stated Dept. of Energy for granting me the magnetic fusion energy technology fellowship for almost 5 years, and thanks are also given to the staff at Oak Ridge Associated Universities for administering the fellowship and approving my travel requests to various scientific meetings. Financial support for travel to scientific meetings was also supplied by the Edge Plasina and Particle Control Group of Oak Ridge National Laboratory. In addition, thanks are given to the North Carolina State University Dept. of Nuclear Engineering for assisting in the replication costs of this thesis, and for giving me a good undergraduate and graduate education. 


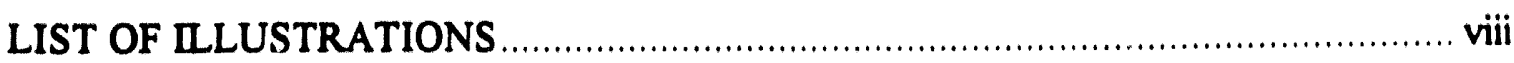

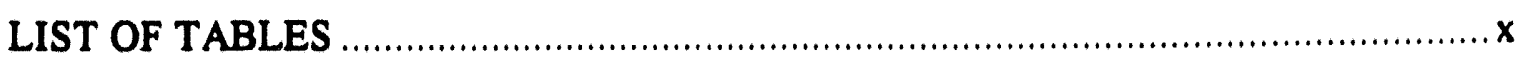

CHAPTER 1. STATEMENT OF PROBLEM

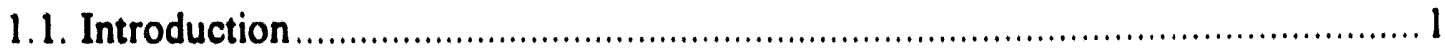

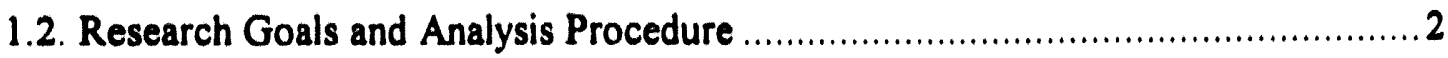

1.3. Interaction Between Neutrals and Plasma in the Tokamak SOL ........................6

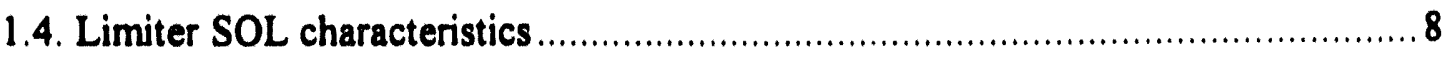

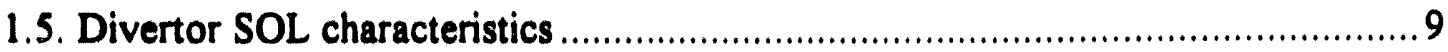

1.6. Simulation of Divertor and Pump-limiter Tokamak Scrape-off-layers .............. 12

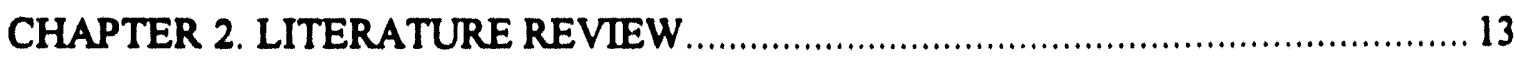

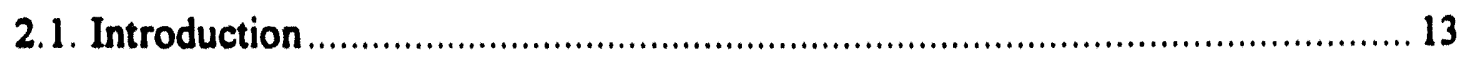

2.2. Two-Dimensional Plasma Models.................................................................. 14

2.2.1. Benchmarks of the b2 Code............................................................... 15

2.2.2. Coupled 2-D Plasma and Neutral Transport Codes ................................ 16

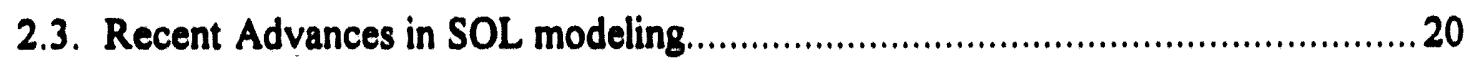

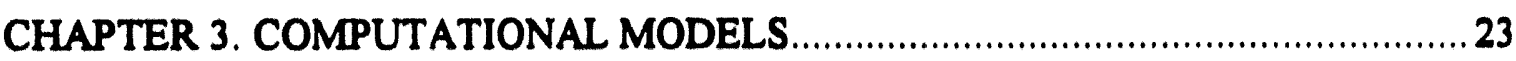

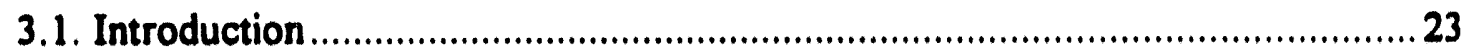

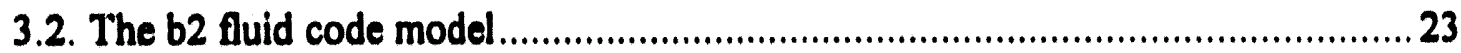

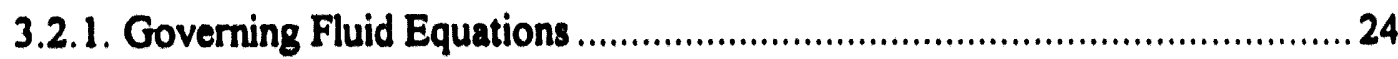

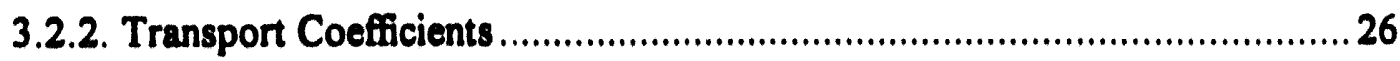

3.2.3. Free-Streaming Limits on Conduction and Momentum ............................. 27

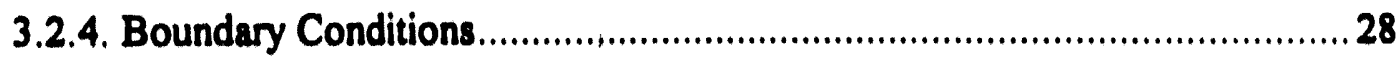

3.2.5. Internal One-Species Recycling Model................................................... 32 
3.3. The DEGAS Monte Carlo Calculation .......................................36

3.4. Coupling Between b2 and DEGAS....................................38

3.4.1. Information Transfer and 2-D Grid....................................38

3.4.2. The Recycling Model Using DEGAS Source Terms....................42

3.4.3. Numerical Coupling and Iteration Procedure ...........................45

3.4.4. System Convergence Diagnostics ....................................46

3.4.5. Physical Regimes of Strong Neutral/Plasma Coupling ..................46

3.5. Two-Species Molecular Physics Recycling Model for the b2 Code............47

3.6. Stairstep Geometry Approximation for Tore Supra Simulation................52

CHAPTER 4. RESULTS AND ANALYSIS ...................................55

4.1. Results for the DIII-D tokamak..............................................55

4.1.1. Coupled b2/DEGAS Simulations .....................................57

4.1.2. Numerical Convergence of Coupled Code Simulations ....................58

4.1.3. Results for the Nine Coupled b2/DEGAS Simulations..................60

4.1.4. Effect of Core Boundary Condition ....................................76

4.1.5. Benchmark to DIII-D Data ...............................................77

4.1.5.1. Low Power Input Shot (5 MW)..................................78

4.1.5.2. High Power Input Shot $(10 \mathrm{MW})$...................................89

4.2. Results of the Tore Supra Simulation..................................... 100

4.2.1. Shot Description and Available Diagnostic Data........................ 100

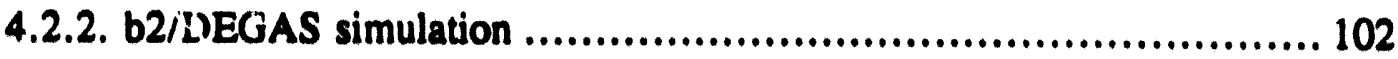

4.2.2.1. Grids and Modeled Region ...................................... 102

4.2.2.2. Input Parameters and Convergence of b2/DEGAS Simulation... 104

4.2.2.3. Calibration of b2 Single-Species Recycling Model ............... 106

4.2.3. Comparison with Experimental Data ................................... 108

4.2.4. Effect of Momentum Flux (Parallel Viscosity) Free-Streaming-Limit 109 
4.2.5. Effect of Pumping on Peak Plate Heat Flux.

5.1. Overview of DIII-D Simulations ...................................................... 114

5.1.1. Coupled b2/DEGAS Simulations ................................................ 114

5.1.2. Molecular Physics Model ............................................................. 115

5.1.3. Effect of Divertor/Wall Pumping ....................................................115

5.1.4. Effect of Ion Conduction Free-Streaming Limit ............................... 116

5.1.5. Use of the Non-orthogonal Grid ................................................... 117

5.1.6. Shortcomings and Sensitivities of Individual b2 and DEGAS Models...... 118

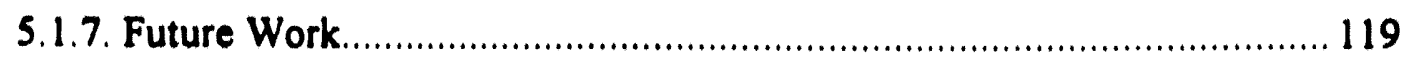

5.2. Overview of Tore Supra Simulation ................................................... 120

5.2.1. Coupled b?:/DEGAS and Calibrated b2 Simulations ............................ 120

5.2.2. Effect of Parallel Viscosity Free-Streaming Limit.............................. 121

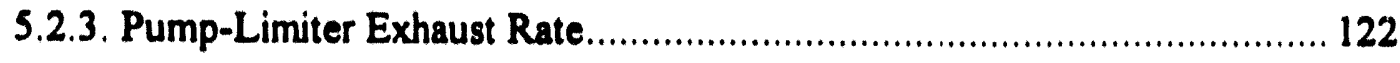

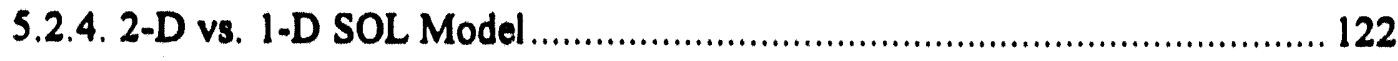

5.2.5. Shortcomings and Sensitivities of Individual b2 and DEGAS Models..... 122

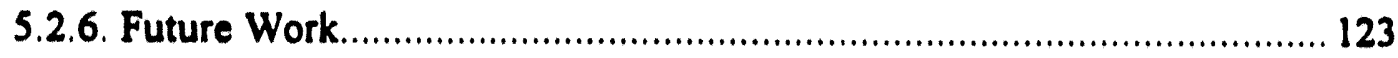

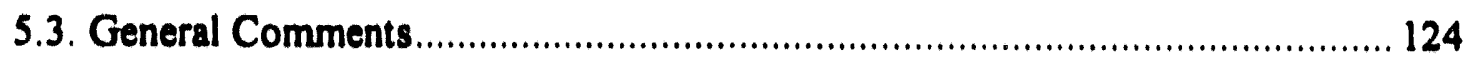

5.3.1. Divertor vs. Pump-Limiter SOL...................................................... 124

5.3.2. One General Code for Plasma-facing-component Analysis ..................... 125

APPENDIX A: Basic Tokamak Physics and Terminology ..................................... 126

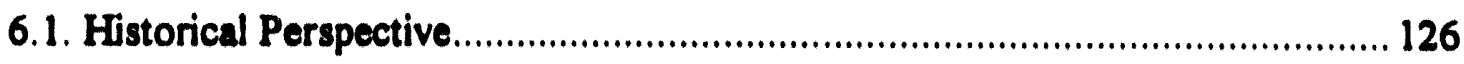

6.2. Core Confinement Regimes of Tokamaks................................................. 126

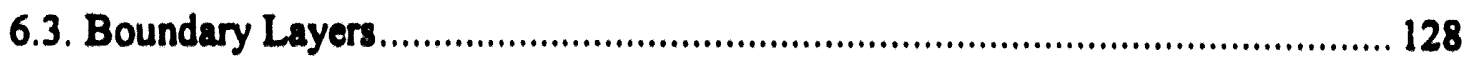

6.3.1. Definition and Basic Physics Description ............................................ 128

6.3.2. Parallel and Perpendicular Transport in the Scrape-off-layer .................. 128

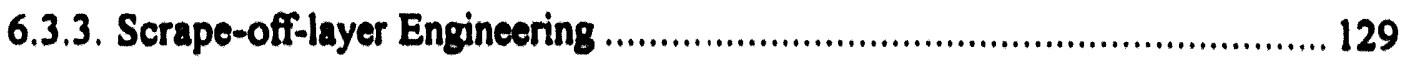


6.4. Plasma-facing-components Configurations

APPENDIX B: Examples of O-D and 1-D SOL Plasma Models 140

7.1. Zero-Dimensional Plasma Models 140

7.2. One-Dimensional Plasma Models 141

7.2.1. One-Dimensional Radial Transport Models.

7.2.2. One-Dimensional Parallel Transport Models 142

APPENDIX C: Non-orthogonal Grid Generation Procedure. 144 APPENDIX D: Detailed Comparisons between the Two-Species Model and b2/DEGAS Codes 146 REFERENCES 156 


\section{LIST OF ILLUSTRATIONS}

Figure

Figure 1: Heat flux correction for tilted target plates.

Figure 2: Schematic of core and edge plasma flows in a diverted tokamak .10

Figure 3: Schematic of poloidal flux surface expansion near the divertnr $x$-point...11

Figure 4: Toroidal view schematic of Tore Supra SOL and outboard pump-limiter.30

Figure 5: Reaction rate coefficients for the following processes .36

Figure 6: Schematic of stairstep approximation of a pump-limiter head .52

Figure 8: Relevant characteristics of shot 66821. .78

Figure 9: 1990 benchmark profiles and comparisons to data .79

Figure 10: Orthogonal grid used for shot 66821 in this research. .80

Figure 11: Non-orthogonal grid used for shot 66821 in this research... .81

Figure 12: Comparisons of (a)plasma density, (b)electron temperature, and (c)plasma heat flux profiles along the outboard divertor plate between experimental data from the low power shot and both the orthogonal and non-orthogonal grid simulations 83

Figure 13: Relevant characteristics of shot 73617 .89

Figure 14: 1991 benchmark measured (top graph) and computed density profiles (bottom graph) along the outboard divertor plate for shot 73617. .90

Figure 15: 1991 benchmark measured and computed temperature profiles along the outboard divertor plate for shot 73617 .. 91 Figure 16: 1991 benchmark measured and computed heat flux profiles along the outboard divertor plate for shot 73617. .92

Figure 17: Comparisons of (a)plasma density, (b)electron temperature, and (c)plasma heat flux profiles along the outboard divertor plate between experimental data from the high power shot and both the orthogonal and non-orthogonal grid simulations 
Figure 18: Location of Langmuir probes in pump-limiter throat ....................98

Figure 19: 2-D telescoping mesh used in the Tore Supra analysis ................. 101

Figure 20: Comparison of toroidal (a)density and (b)temperature profiles for the b2/DEGAS and calibrated b2 runs at a distance $0.4 \mathrm{~cm}$ radially below the limiter head 105

Figure 21: Plasma density profile in the pump-limiter throat for the b2/DEGAS run and the calibrated b2 model 105

Figure 22: Radial profiles of (a)plasma density, (b)temperature, and (c)heat flux along the plate for the coupled b2/DEGAS simulation and the calibrated b2 model.107 Figure 23: Toroidal profiles of (a)plasma density and (b)pressure in the throat for the calibrated b2 model with and without the parallel viscosity free-streaming-limit at a radius of $0.4 \mathrm{~cm}$ 110

Figure 24: Comparison of line-averaged density for Tore Supra for shots 3126 and 3127 112

Figure 25: Radial density profiles with and without active pumping 113 Figure 26: Schematic of core plasma and edge flasma boundary and the last-closedflux-surface (LCFS) of a typical limiter and divertor tokamak 131

Figure 27: Dependence of the electron impact ionization reaction rate coefficient (hydrogen) on the electron temperature (approximated) .......................... 131

Figure 28: Illustration of the various types of plasma-wall contact ................ 133

Figure 29: Schematic of open and closed pump-limiter configurations.............. 134

Figure 30: Schematic of the conceptual INTOR poloidal divertor. ................. 135

Figure 31: Diagram of a single-null divertor (PDX) and a double-null divertor (ASDEX). 135 


\section{LIST OF TABLES}

Table

Table 1: Summary of Transport Coefficients for Various Devices. 19

Table 2: b2/DEGAS Coupled Simulations Parametric Variations for DIII-D 56

Table 3: Numerical Convergence Diagnostics for Simulation 1(DIII-D) 58

Table 4: Comparison of Divertor Plate Plasma Parameters (Simulation \#1) 62

Table 5: Comparison of Divertor Plate Plasma Parameters (Simulation \#2) 64

Table 6: Comparison of Divertor Plate Plasma Parameters (Simulation \#3) 65

Table 7: Comparison of Divertor Plate Plasma Parameters (Simulation \#4) 66

Table 8: Comparison of Divertor Plate Plasma Parameters (Simulation \#5) 67

Table 9: Comparison of Divertor Plate Plasma Parameters (Simulation \#6) 68

Table 10: Comparison of Divertor Plate Plasma Parameters (Simulation \#7) 69

Table 11: Comparison of Divertor Plate Plasma Parameters (Simulation \#8) ................ 71

Table 12: Comparison of Divertor Plate Plasma Parameters (Simulation \#9) ................. 74

Table 13: Comparison of Core Power Efflux for Simulations 1-9 ............................. 75

Table 14: Input Parameters for DIII-D Low Power Shot Benchmarks......................... 86

Table 15: Input Parameters for DIII-D High Power Shot Benchmarks ......................... 95

Table 16: Input Values in the b2 Code for the Tore Supra Simulation ......................... 102

Table 17: Convergence Diagnostics for b2/DEGAS Simulation (Tore Supra).............. 102

Table 18: Comparison of Measured Values and Modeling Results for Tore Supra ........ 106

Table 19: Variation of Recycling Coefficients for Tore Supra Simulation ................... 111

Table 20: Comparison of Recycling Models for Simulation 1 ................................. 143

Table 21: Comparison of Recycling Models for Simulation 2 ................................... 144

Table 22: Comparison of Recycling Models for Simulation 3 .................................... 145

Table 23: Comparison of Recycling Models for Simulation 4 ................................. 146 
Table 24: Comparison of Recycling Models for Simulation 5

Table 25: Comp: rison of Recycling Models for Simulation 6 148

Table 26: Comparison of Recycling Models for Simulation 7 149

Table 27: Comparison of Recycling Models for Simulation 8 150

Table 28: Comparison of Recycling Models for Simulation 9 


\section{CHAPTER 1}

\section{STATEMENT OF PROBLEM}

\subsection{Introduction}

Recent experiments on present-day magnetically-confined nuclear fusion devices (tokamaks) have shown that the major technological hurdles to overcome in the operation of fusion reactors are related to the interactions occurring at the plasma/material boundaries (plasma-facing-components). One major problem is that the core plasma fusion power levels envisioned in steady-state reactor operation will place larger heat loads at the surfaces of plasma/wall contact than can be handled by present first wall candidate materials designs. Accurate predictions of the peak power load, called the peak heat flux, to the surfaces of plasma/material contact are crucial for this reason. Safety factors are necessarily introduced in the peak heat flux calculation for plasma-facing-component design to allow for inaccuracies in the models used in plasma simulations. One of the main goals of this study is to devise a model or a set of models which will improve predictions of peak heat flux to the target plates in all regimes, which should allow for smaller safety factors in design of plasma-facing-components for power-producing reactors. A related problem in present-day devices is the need for control of the plasma density near plasmafacing-comr onents. Peak heat flux (and plate erosion) is lowest for a low-temperature plasma in contact with plasma-facing-components (the edge or scrape-off-layer plasma, as opposed to the core plasma). If the electron temperature falls too low, however, migration of cold impurity and fuel neutrals generated from plasma/wall interactions from the wall through the scrape-off-layer (SOL) plasma and into the core plasma can be enhanced. In this case, core radiation losses are increased, dropping the core plasma temperature and the core fusion power density. Hence a density/temperature regime must be achier ed in 
the edge plasma in which a balance between plate erosion and density control requirements is maintained. A qualitative and elementary description of edge plasma physics and terminology is included in the appendices for the reader unfamiliar with the subject matter. An introduction to limiters and divertors is also included in the appendices.

\subsection{Research Goals and Analysis Procedure}

The research goals can be summarized as follows. First a method to model the tokamak SOL is desired, taking into account in a detailed manner the coupling between the plasma and neutral species. Accurate calculation of target heat and particle flux are the main parameters of interest in this study. Next, it is desired to benchmark these calculations against experimental data from the DIII-D divertor tokamak and the Tore Supra pump-limiter tokamak to obtain "best-fit" transport coefficients. Finally, it is desired to examine the effect of ion conduction and momentum flux free-streaming-limits on plasma parameters, as well as the effect of non-orthogonal magnetic flux surface and target plate geometry. Each goal is discussed in detail below.

Accurate prediction of peak heat flux in all operating regimes is especially difficult because the tokamak SOL contains both plasma and neutral atomic and molecular species; the physics governing one species is distinct from but strongly coupled to the other species. Solution of a model including the detailed physics for both species and the coupling between the species may not be viable with present numerical techniques. The plasma can be modeled as a fluid but the neutrals are most accurately modeled by single particle kinetic simulations (e.g. Monte Carlo). Even without treatment of the neutrals, solution of the highly non-linear plasma fluid equations usually requires iterative methods. Hence, present day two-dimensional (2-D) modeling of the tokamak SOL usually consists of a detailed model for one species and a simple model for the other species. However, simplifications of the model for either species reduces the magnitude of the coupling between the species and can lead to inaccurate predictions of SOL parameters, including peak heat flux to plasma-facing-components. As mentioned above, peak heat flux is 
especially important because it is the critical factor in determining the survival probability of target plates. This research develops a method to use the best available models for both species through the external coupling of two existing codes, 62 (plasma transport) ${ }^{1}$ and DEGAS (neutral transport) ${ }^{2}$. The b\% code has been modified to allow the use of source profiles computed by the DEGAS cocle, and to write out the plasma density and temperature profiles into a DEGAS input file. The DEGAS code is used to compute neutral/plasma source profiles from the plasma profiles supplied by the b2 calculation. Without the coupling, the default recycling model in b2 can not accurately model the molecular physics or charge exchange, and the DEGAS model does not compute the edge plasma parameters changes caused by changes in the recycling source distributions. A similar technique using the same codes has been used to model the Burning Plasma Experiment (BPX) tokamak study ${ }^{3}$ and the Tokamak Fusion Test Reactor (TFTR) device 4 . The latter analysis benchmarked the model simulations to available experimental data for the limiter SOL of TFTR. New results presented here are benchmarking against experimental data from two operating devices with different plasma-facing-components: a pump-limiter SOL (the Tore Supra tokamak) and a divertor SOL (the DIII-D tokamak). The divertor SOL is generally in a higher plasma density and lower temperature regime than the pump-limiter SOL. Emphasis is placed on the plasma modeling results for the divertor simulations in this work.

Accurate prediction of the particle flux profile along the plasma-facingcomponents is also a difficult problem since particle flux depends sensitively on the recycling conditions at the plasma/wall interface. The particle flux profile is crucial in determining pumping requirements for control of impurity and fuel neutrals in the edge plasma. Hence the other main objective of this study is to accurately compute the particle flux profile along plasma-facing-components. Precise calculation of both the particle and heat flux profiles entails a good estimate of both the plasma density and temperature in the edge plasma. 
Other deficiencies exist in present day models for the tokamak SOL plasma. While present SOL plasma simulations no longer require the use of Cartesian grids, most simulations still require orthogonal grids, i.e. with the targets normal to magnetic flux surfaces. The peak heat flux at the target surface is estimated by multiplying the computed heat flux by the cosine of the incident angle flux surfaces make with the true target (fig. 1).

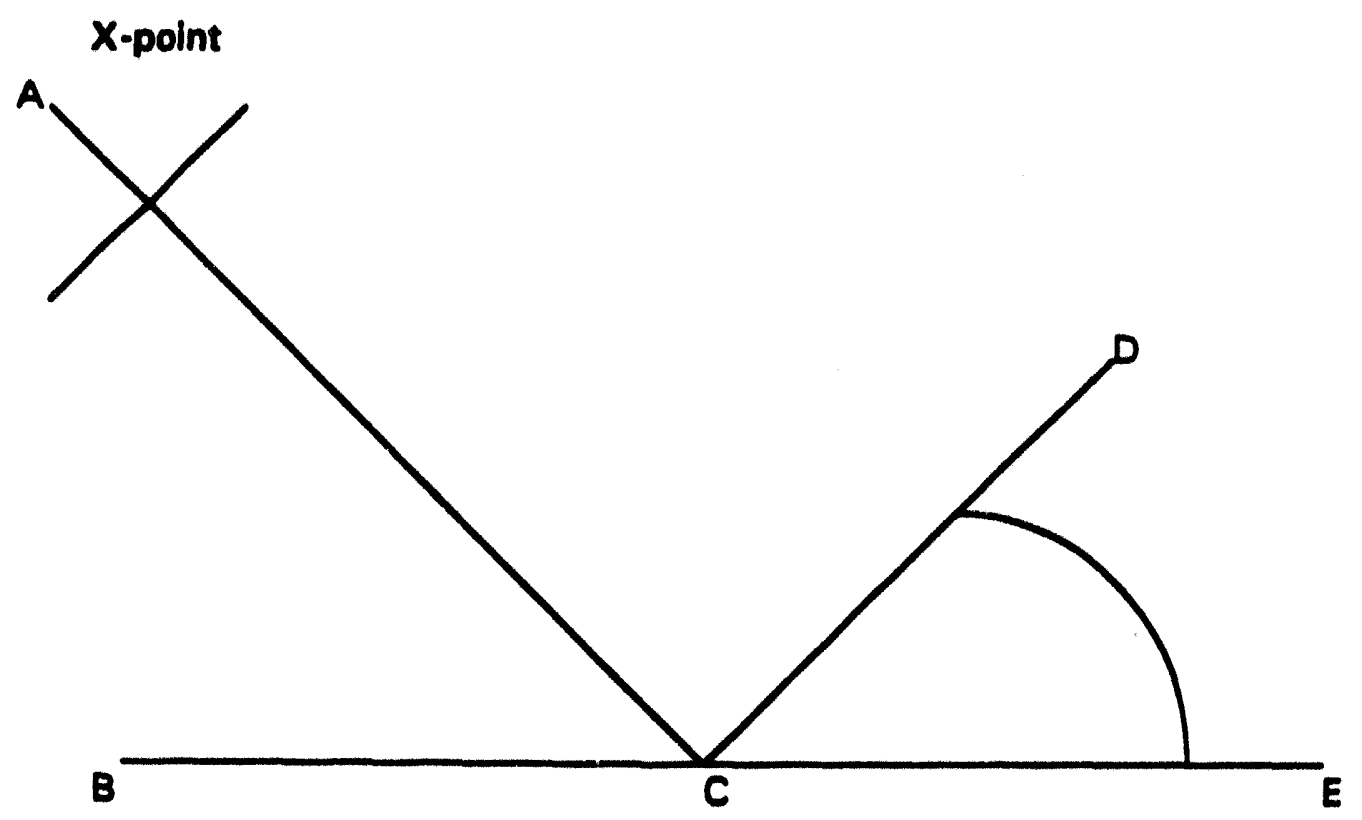

Figure 1: Heat flux correction for tilted target plates. AC represents the separatrix, $C D$ represents a plate orthogonal to the separatrix, $\mathrm{CE}$ represents an outward tilted plate. The heat flux computed with the orthogonal geometry is multiplied by the cosine of angle DCE to obtain the projected heat flux on the tilted target.

This extrapolation has a large uncertainty because the location of the separatrix and the exact angle at which field lines impact the target are not precisely known. This research presents an alternative to the aforementioned extrapolation, the non-orthogonal grid (grid compression) method, and discusses a second alternative, the stairstep method. The gridcompression method distorts the grid in the region of the target plates to approximate true 
geometry; orthogonality is not maintained. A non-orthogonal grid generation algorithm was written and implemented as part of an existing orthogonal grid generation packages which generates grids based on magnetic flux measurements of the DIII-D tokamak. The generation of the non-orthogonal grid entails compression of the part of the orthogonal grid which lies beyond the physical target plate. A similar grid compression tachnique has been used by Coster in 2-D plasma modeling of TFTR. The stairstep method maintains grid orthogonality by approximating the target as a stairstep. Application of the stairstep method is problem specific because the user must specify where in the physical domain that slanted material surfaces occur and block out the calculation inside the material surface. Both methods should provide a more accurate determination of the particle and heat flux to the target than the conventional extrapolation from orthogonal grid calculations.

One final issue considered here is the use of kinetic-based free-streaming-limits on ion conduction and momentum flux in the plasma modeling portion of the analysis. Ion conduction is limited as a fraction of the ion particle flux times the ion acoustic speed. Comparison and discussion of modeling results with and without the use of the ion conduction free-streaming-limit is given in the DIII-D analysis. Application of the momentum flux free-streaming-limit is examined in the Tore Supra simulation.

The chronological steps taken in analyzing different operating regimes of divertor and limiter SOL plasmas were :

1. The b2/DEGAS set was used to model a particular SOL regime

2. After convergence of the b2/DEGAS coupled code simulation, free parameters in the b2 recycling model(s) were "calibrated" to match the b2/DEGAS results. The default recycling model was used for Tore Supra and a now two-species molecular physics model was implemented for DII-D (noe below). 
3. The b2 code was then used to model two DIII-D discharges (5 MW and $10 \mathrm{MW}$ input single-null with the "calibrated" molecular physics model) and one Tore Supra discharge (1.8 MW input with the "calibrated" default recycling model).

Because of the shortcomings of the default b2 recycling model, a new molecular physics model, based on a recent model by Hogan (private communication or APS92), was implemented. The advantage of the new model is that fewer parameters have to be varied over the different scenarios to match coupled b2/DEGAS results.

Important SOL physics is described in chapter 1. The physics discussion will elicit the density/temperature regimes in which coupling between the plasma species and the neutral species (and also the two codes) is very strong, necessitating the use of the coupled codes. Chapter 2 contains a literature survey of work done in the area of 2-D SOL modeling (with and without detailed neutral tranaport calculations) in the last decade, with a brief discussion of the simplification to 1-D models and representative applications. Chapter 3 contains a discussion of the models used in the two primary analysis codes, b2 and DEGAS, as well as changes made to those models in the course of this research and the difficulties in coupling the two codes. Chapter 4 discusses results of the modeling and benchmarking efforts to experiments on both the Tore Supra and DIII-D tokamaks, and chapter 5 discusses the effectiveness of the models, and makes recommendations for future work.

\subsection{Interaction Between Neutrals and Plasma in the Tokamak SOL}

As mentioned previoualy, the governing plasma continuity, momentum, and energy balance equations are intimately coupled to the atomic physics and plasma/wall processes that describe the recycling of plasma from the material boundaries (described below). The discussion is relevant because the amount of reoyeling in the SOL may strongly affect the peak heat flux to the target plates and other SOL plama parameters.

When ions strike a material surface such as limiter or divertor neutralizer plates, the ions can diffuse into the bulk material (the dominant process) or backscatter as 
energetic neutrals. A backscattered neutral has a fraction of the thermal energy of the incident ion plus the kinetic energy gained by the incident ion falling through the electrostatic sheath potential drop. In general, these neutrals may have sufficient kinetic energy to travel through the SOL without being ionized, and they can present a potential energy loss mechanism to hot core ions via CX.

In the case of diffusion into the target lattice, ions are neutralized rapidly after impact and hydrogenic species recombine with a free neutral atom or impurity radicals. Neglecting impurities, the newly formed molecule diffuses toward the target surface and causes desorption of an existing surface molecule at the wall temperature, usually a fow hundred degrees Celsius. The desorbed molecule then directly or through multiple steps dissociates into two particles (an ion and a neutral, or two neutrals) of a fow $O V$ energy, The neutrals can undergo electron impact ionization (EII) or CX with ions near the target. The En process drains $25 \mathrm{eV}$ on the average per ionization from the electron fluid; the loss in a given SOL region depends on the local electron temperature. In addition, the ion born from EII has the Franck-Condon energy, representing a particle and energy source for the local ion tuid. Conversely EIl represents a particle sink for the neutrals. FranckCondon neutrals that charge exchange(CX) with local ions represent an ion energy sink if the local ion temperature is above the Franck-Condon energy, which is usually true for a large tokamak SOL. This ion energy sink takes energy out of the local ion fluid and deposits it at the wall or moves it to where CX neutrals are ionized. However, cold neutrals can aleo initiate a series of CX processes, at the end of which a hot core plasma ion becomes neutralized and travels across magnetic field lines, possibly reaching the vessel wall. The presence of impurities can result in significant amounts of energy being radiated in the SOL.

A fraction of ions striking material boundaries are absorbed. The fraction of these ions which are re-emitted as neutrals into the SOL at the target plates is given by the plate recycling coefficient, $R_{p}$. The ion flux parallel to the magnetic field atriking the target and 
diffusing into the first few atomic mono-layers rapidly saturates gaps in the molecular iattice. Hence, $R$, approaches unity very quickly at the target plates. Because the crossield ion fux is much smaller than the parallel ion flux and the first wall has a much larger surface area than the target plates, the cross-field ion flux to the first wall does not saturate all of the gaps in the first fow mono-layers of the first wall. Hence the wall recycling coefficient, $R_{w}$, cun be 0.5 for $\$$ well-conditioned first wall, i.e. free of hydrogen and impurities and containing a large number of vacant lattice sites which can be occupied by incident ions. Conversely first walls can have $R_{w}$ greater than unity if large numbers of molecules are retained in the first fow mono-layers; these "dirty" first walls can feed particles into the SOL (outgessing). The importance of the recycling coefficient can be shown as follows: each ion striking the plate has a probability of $R$, of not being absorbed and coming back into the SOL as a neutral and eventually being reionized. The first generation of recycled particles, $R$, times the original plate ion flux, again has a probability of $R$, of coming back into the SOL after being re-neutralized at the plate. Assuming $100 \%$ efficiency in SOL ionization, $R$, less than 1.0, and a constant radial influx of plasma from the core into the SOL, and summing over an infinite number of possible ion generations, the maximum plate flux is given by:

$$
\Gamma_{1}^{\infty}=\frac{\Gamma_{1}}{1-R_{1}}
$$

For a plate recycling coefitcient of 0.99 , the suturation ion flux striking the plate is 100 times larger than the ion flux with no recycling. This phenomenon is known as fux amplification and $1 /\left(1-R_{0}\right)$ is the entuntion or maximum lux amplification factor, $a_{x y}$ The efiective amplification fhctor, $a_{y}$, depends on SOL ionization efficiency, as well a $R_{p}$. Fux amplification can greatly increase the SOL neutrals avalable for local ionization and CX, which increnses the magnitude of the sorementioned source terms and strengthens the coupling between the plamm and the neutrals. 


\subsection{Limiter SOL characteristics}

Limiters in present day tokamaks are usually placed at shallow incidence angles to the parallel magnetic field direction to spread out the heat flux. This placenent increases the effective surface area over which energy is deposited on the limiter plates by $1 / \cos (\alpha)$, where $\alpha$ is the angle of incidence of the magnetic field on the targets; $\alpha$ is on the order of a fow degrees or less. Consequently the heat flux is reduced by roughly $\cos (\alpha)$ is compared to normal incidence. To generate a cold, dense SOL, however, the recycling sources must be localized near the targets. The core plasma is usually too close to the limiter to allow plasma temperature to fall and plasma density to rise to operate at a high ionization efficiency. Hence the limiter SOL usually operates in the low density and medium temperature range.

Addition of a pump to a limiter system can significantly alter SOL characteristics. Use of a pump is desirable to remove impurities and neutrals generated near the targets by plauma-wall interactions. Aseo, pumping can be used to control the SOL density, which in turn affects the core density. The pump-limiter removal efficiency, $\eta_{\mathrm{mom}}$ is defined as the fraction of the particles that hit the plate and are removed by the pump. Athough the actual removal efficiency depends on various conductances, pumping speed, and neutral pressure, the design $\eta_{\mathrm{mm}}$ of the Tore Supra outboard pump-limiter module is $50 \%$. It should be noted that the removal efficiency defined here differs from the commonly used exhaust efficiency, which is defined as the total fraction of the core efflux that is removed by the pump-limiter. Removal of neutrals by the pump effectively reduces $R_{p}$, and the effective recycling coefficient is given by:

$$
R_{f}^{\prime}=1-\eta_{m}
$$

For a limiter with $R$, near unity and $\eta_{m}$ of $30 \%$, the effective recycling coefticient is approximately 0.5 . This reduction of $R$, prevents a large flux amplification factor and the large associated recyeling sources. Hence the pump-limiter plama parameters usually do not achieve the desired cold, dense SOL regime. 


\subsection{Divertor SOL chanacteriatics}

Magnetic diveror target plates are at graxing incidence angles to the parallel magnetic field for the same reason as limiter target plates are: spreading out of the heat Aux. One major advantage the divertor has over the limiter is physical separation of the core from the target plates. The SOL plauma flows to the magnetic x-point and then is diverted away from the core plasma toward the target plates (fig. 2). The core efflux transports particles and energy into the SOL, which maintains a high SOL plasma temperature above the X-point. Below the x-point, however, there is not a significant radial flow of particles and energy from the private-flux-region into the SOL. Energy is conducted and convected from the upper part of the SOL (above the x-point) to the lower part (below the x-point), and particles are transported from the upper part to the lower part via a Aluid dow velocity (determined by momentum balance). Because of finite thermal conductivity, the plama temperature drops along fux surfaces between the $x$ -

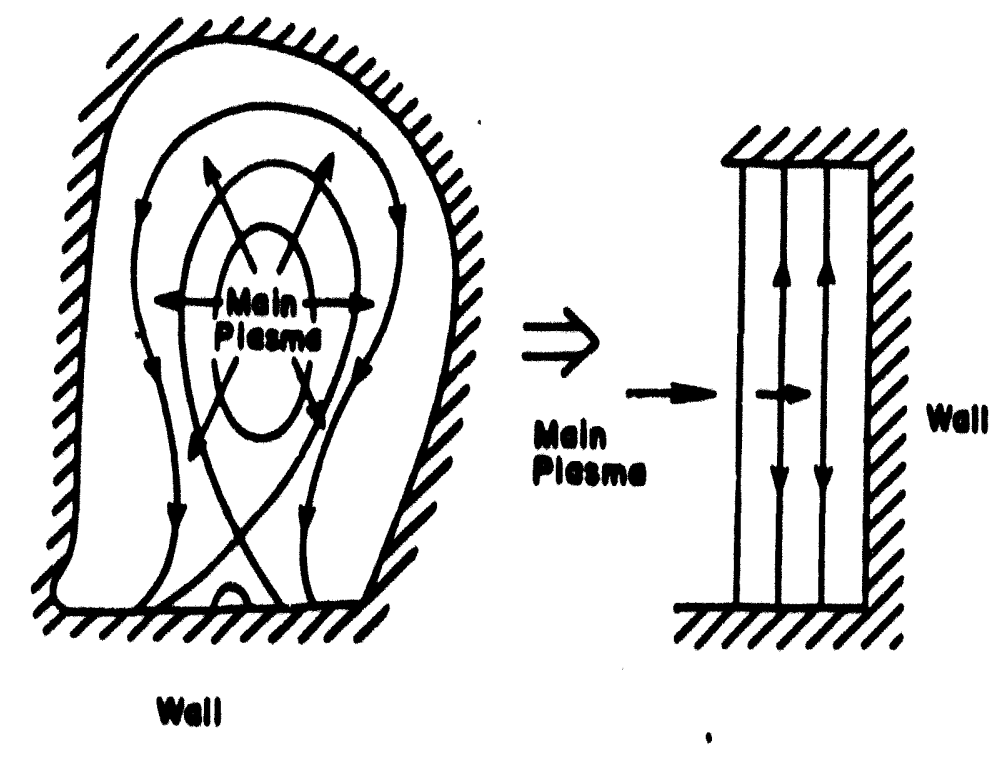

Figure 2: Schematic of core and edge plama towe in a diverted tokamak, taken from Ponts 
point and the plate. The plasma temperature can easily drop by a factor of 5 between the $x$-point and the plate for common operating conditions on the DIU-D tokamak, for example. Henre plasma density can increase by a factor of 5 also. The physical separation between the core and targets thus aids in the buildup of a cold, dense SOL.

As shown in 68. 3, the divertor configuration magnetic flux surfaces expand radially near the X-point, as compared to the midplane. Correspondingly the SOL radial thickness (radial e-folding length) also expands near the x-point, and neutrals born from plasmatarget plate interactions have a high probability of being ionized before reaching the x-point. Recycling sources become localized, which increases $n_{p}$ and decreases $T_{0}$ below the x-point. Divertor operation, therefore, generally achieves a much higher flux amplification factor than limiter operation.

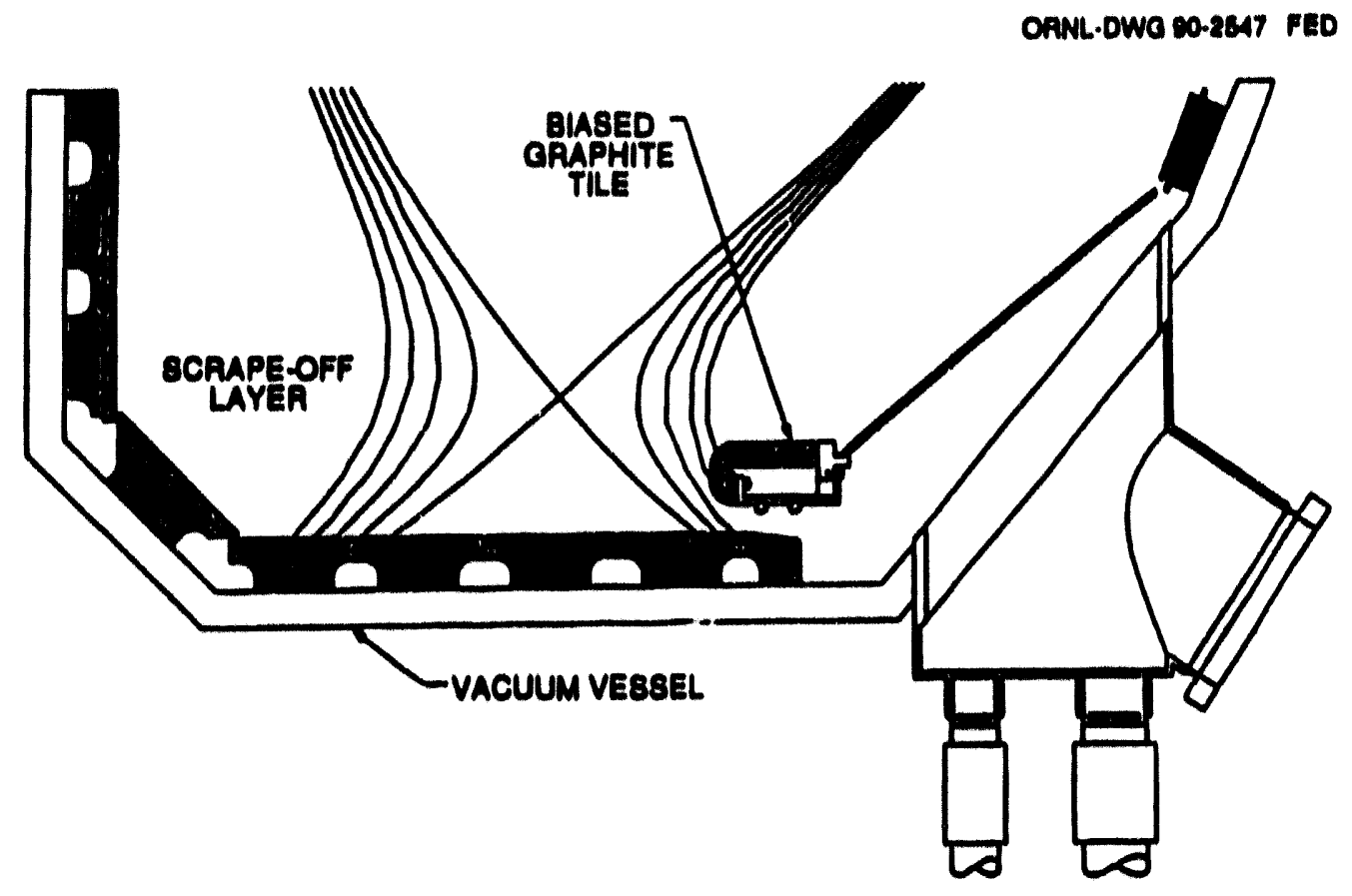

Figure 3: Schematic of poloidal Alux wurfice expansion near the divertor x-point (ORNL drawing 90-2457 FED) 
benchmarking of coupled 2-D codes for the SOL plasma (b2) and neutral species (DEGAS), using the same codes used in this analysis. One recent simulation of DIII-D data used a different plasma code, PLANET, coupled to the same neutral transport code used in this research, DEGAS.

Relevant physics not in the version of the b2 code used in this analysis has been added recently to 2-D SOL plasma models, including E X B drifts, electrostatic potentials, transient analysis, and inclusion of impurities. Investigation of the use of non-orthogonal grids for SOL plasma simulations with more precise solution techniques than present numerical methods is commencing now at UCLA. These topics are not investigated in this research but will be discussed in the context of ongoing and future improvements to the present codes.

\subsection{Two-Dimensional Plasma Models}

Two-dimensional plasma models also offer a wide range of complexity. These models generally use subset of the general equations6, derived specifically for the geometry needed, i.e. toroidal geometry. Benchmarks of the b2 code to experimental data are examined in this section.

Benchmarking of most 2-D codes usually involves selecting values for the radial anomalous transport coefficients which best fit experimental data. All of the 2-D benchmarking of present tokamaks is relevant because it provides a range of radial transport coefficients which must be considered in the coupling of the b2 and DEGAS codes described in the next chapter. Unfortunately, the freedom to vary these coefficients to fit data has the drawback that a good fit with a particular set of values might actually maak relevant physics and make otherwise unsuitable transport coefficients seem reasonable. In the cases below where the plasma codes are used in stand-alone mode, the relevant physics would occur in the coupling of the neutrals to the plasma, which most of the codes do in very elementary ways, if at all. Hence usage of the "best fit" transport coefficients from present experiments in simulations of future devices such as ITER may 
yield inaccurate predictions of ITER target plate parameters. The b2 code has been used to model many planned devices and paper studies: NTTOR $^{\text {, }}, \mathrm{TCFX}^{1}, \mathrm{BPX}^{3}$, and ITER $9,10,11$. Only the modeling of BPX will be discussed here, since one recent analysis involved coupling of the b2 and DEGAS codes.

\subsubsection{Benchmarks of the b2 Code}

The $b 2$ code with its internal recycling model has been used extensively to model present and planned fusion devices. The governing equation set is listed and discussed in the computational models section in Chapter 3. Present devices which the b2 code has been used to model include ASDEX, DIII-D and JET.

In the ASDEX simulation ${ }^{12}$, two sets of transport coefficients yielded the best fit to data from Langmuir probe for ohmic shots. Near the separatrix, steep gradients in the plasma density and temperature were matched by using $\chi_{1}^{\prime}=1.5 \mathrm{~m}^{2} / \mathrm{s}, \chi_{1}^{\prime}=0.2 \mathrm{~m}^{2} / \mathrm{s}$, $D_{\perp}=1.0 \mathrm{~m}^{2} / \mathrm{s}, R_{p}=0.80$, and $v_{\text {comv }}=0$. However the gradual decay of the plasma density and temperature in the outer part of the SOL was best matched by using $x_{1}=5.0 \mathrm{~m}^{2} / \mathrm{s}$ and $D_{1}=3.0 \mathrm{~m}^{2} / \mathrm{s}$. Braams indicated 7 that the larger transport coefficient lie in the upper end of the range observed at ASDEX.

Rensink benchmarked ${ }^{13}$ b2 calculations to Langmuir probe and infrared television camera (IRTV) data from the DIII-D divertor floor. In low confinement mode (L-mode) operation, the best fit to data came from using the following transport coefficients : $\chi_{1}=4.0 \mathrm{~m}^{2} / \mathrm{s}, \chi_{1}=0.2 \mathrm{~m}^{2} / \mathrm{s}, D_{1}=1.0 \mathrm{~m}^{2} / \mathrm{s},=0.80$, and $v_{\text {couv }}=0$. In contrast, fit of high confinement mode (H-mode) operation required the following transport coefficients : $\chi_{1}=1.0 \mathrm{~m}^{2} / \mathrm{s}, \chi_{1}^{\prime}=0.2 \mathrm{~m}^{2} / \mathrm{s}, D_{1}=1.0 \mathrm{~m}^{2} / \mathrm{s}, R_{p}=0.90$, and $v_{\text {com }}=-40 \mathrm{~m} / \mathrm{s}$. The quality of the fit was good, although the Langmuir probe data indicated a steeper initial radial falloff of the plasma density and temperature at the target in $\mathrm{H}$-mode than predicted by the simulation (fig. 10, chapter 4). A more recent simulation 14 of data from DII-D high heat flux experiments using the aforementioned transport coefficients from H-mode operation concluded that the predicted divertor heat flux and temperature were much higher than 
experimentally observed by the IRTV when separatrix plasma density and temperature from Thomson Scattering measurements near the outer midplane were matched by the simulation. The transport coefficients used in the simulations of DIII-D in chapter 4 are taken largely from Rensink's work.

Prinja modeled 15 JET SN discharges and concluded that the best fit to low power input experimental data was given by the following transport coefficients: $\chi_{1}=1.0 \mathrm{~m}^{2} / \mathrm{s}$, $\chi_{1}=0.33 \mathrm{~m}^{2} / \mathrm{s}, D_{1}=0.33 \mathrm{~m}^{2} / \mathrm{s}$, and $v_{\text {conv }}=-20 \mathrm{~m} / \mathrm{s}$. The recycling coefficient appeared to be held fixed in this simulation. Unfortunately the observed in/out power asymmetry observed in JET discharges, in which $25 \%$ more power is dissipated along the inner divertor $\left(P_{i n}\right)$ than the outer divertor $\left(\mathrm{P}_{\text {out }}\right)$, could not be replicated by the simulation.

\subsubsection{Coupled 2-D Plasma and Neutral Transport Codes}

Sugihara solved a set of fluid equations ${ }^{16}$ similar to the ones used in the $b 2$ code without the viscosity terms and computed the plasma-neutral interaction sources with a Monte Carlo code. The work employed a Cartesian mesh for the divertor region and was applied to the INTOR study edge plasma. The work is mentioned here as one of the first methods to use 2-D coupled codes to solve for the SOL plasma and neutral particle parameters, but will not be discussed here because it was not applied to an existing experiment and oversimplified the geometrical effects of the poloidal divertor through the Cartesian mesh approximation.

Igitkhanov solved a set of transport equations very similar to the b2 equations except that the ion viscosity term in the energy equation was neglected. The neutral transport was computed initially by a diffusion approximation and later upgraded 17 to a Monte Carlo technique. Carbon impurities were taken into account in a simple manner, and it was shown that these light impurities were localized to the divertor volume because of friction with the fuel ions.

Ueda solved a set of fluid equations ${ }^{18}$ equivalent to the formulation neglecting all viscosity terms in the b2 code by the particle-in-cell (PIC) method. The source terms 
resulting from neutral-plasma interaction were computed by a 2-D Monte Carlo code ${ }^{19}$ and unity recycling. The atomic reactions modeled by the code were electron impact ionization and charge exchange for hydrogen, and the molecular physics included ionization of molecular hydrogen and dissociation of the molecular hydrogen ion. The Monte Carlo calculation was employed every few tenths of the time needed for convergence of the fluid calculation to a steady-state solution, i.e. 2-5 times. The model was applied to the DIII-D divertor discharges with a line-averaged density of $3 \times 10^{19}$ $\mathrm{cm}^{-3}$ and a power input of $1 \mathrm{MW}$. The grid used in the simulation was non-orthogonal and accurately reflected the magnetic field and divertor target geometry. The particle flux from the core plasma into the SOL was varied to match electron temperature measurements at the outer midplane and divertor targets, and the heat flux input from the core plasma was set by the total experimental power input. Overall the experimental results were encompassed by code predictions for three assumed levels of particle flux input from the core into the SOL, with the highest particle flux used providing the closest fit to measured values. The transport coefficients yielding the best fit were $\chi_{\perp}^{\prime}=\chi_{\perp}^{\prime}=2 D_{\perp}^{B}$, and $D_{\perp}=0.5$ $D_{\perp}^{B}$, where $D_{\perp}^{B}$ is the Bohm diffusion coefficient and is given by :

$$
D_{L}^{B}=6.25 \times \frac{T_{c}}{B_{L o t}}\left(\mathrm{~m}^{2} / \mathrm{s}\right)
$$

As the particle flux into the divertor is increased, the electron temperature profile broadens and the peak shifts off the separatrix; this phenomenon is qualitatively reflected by the Ueda simulation. The only problem with the match to data was that the electron temperature at the plate was best modeled by the mid-range assumed particle flux across the separatrix but the divertor temperature was best modeled with the highest assumed particle flux. Unfortunately no comparisons were made to either density measurements at the midplane and the outer target or heat flux measurements at the target; these measurements may not have been available. 
Petravic used the PLANET code coupled to the DEGAS neutral transport code to model low power discharges on the DIII-D tokamak 20 . The equations used in the PLANET code are similar to the formulation of the b2 code without the viscosity terms. The best fit to divertor plate Langmuir probe data before the DIII-D walls were covered with graphite was achieved by using $\chi_{\perp}=1.0 \mathrm{~m}^{2} / \mathrm{s}$ and $D_{\perp}=0.25 \mathrm{~m}^{2} / \mathrm{s}$, assuming uniform heat and particle fluxes entering the SOL plasma from the main plasma boundary on both the inboard and outboard sides. To model DIII-D data after graphite was introduced into the walls, Petravic found it necessary to assume zero particle and energy fluxes entering the inboard SOL and uniform fluxes entering the outboard SOL. This was necessary to reproduce the in/out power asymmetry of 1:5 observed on some shots of the DIII-D tokamak. The transport coefficients yielding the best fit were $x_{\perp}=4.0 \mathrm{~m}^{2} / \mathrm{s}$ and $D_{\perp}=0.5$ $\mathrm{m}^{2} / \mathrm{s}$, and an anomalous inward pinch velocity was not required to obtain a good fit to data.

Coster externally coupled the b2 and DEGAS codes in the analysis ${ }^{4}$ of TFTR data. The numerical procedure was to run the b2 code until convergence, to run the DEGAS code to obtain the neutral sources, to run the b2 code again until convergence again, and then iterate between the codes until convergence of system diagnostics was achieved. The only system convergence diagnostic used in the model was the integrated plate ion flux to the limiter, calculated by b2. The physical tilt of the limiter surface with respect to flux surfaces was handled approximately in the simulation: the mesh was distorted from orthogonality only very near the limiter. Parallel transport was computed properly because the mesh in the poloidal plane was still tied to magnetic flux surfaces. Moreover the volume of the distorted grid elements was computed properly. The distortion from orthogonality caused the radial transport calculation to be slightly inaccurate only near the limiter, where parallel transport dominates perpendicular transport anyway. Regimes corresponding to very low power input and very high power input were successfully modeled numerically by the coupled code set for TFTR, using a recycling coefficient of 
0.9. The best fit to data available from the experiment, however, was obtained with extremely high values of radial transport coefficients : $\chi_{1}^{\prime}=12 \mathrm{~m}^{2} / \mathrm{s}, \chi_{1}=8 \mathrm{~m}^{2} / \mathrm{s}$, and $D_{\perp}=8$ $\mathrm{m}^{2} / \mathrm{s}$. These transport coefficients are higher than the presently used transport coefficients in most 2-D SOL models by about a factor of 4 . A summary of the best fit transport coefficients obtained from the various benchmarks of the devices discussed above is presented below. Omitted values indicate that information about the omitted quantity was not available.

Table l: Summary of Transport Coefficients for Various Devices

Simulation Description

DIII-D (Petravic 10\% C wals)
DIII-D (Petravic 40\% C walls)
DIII-D (Ueda)
DIII-D L-mode (Rensink)
DIII-D H-mode (Rensink)
ASDEX (separatrix)
ASDEX (outer SOL)
TFTR

$\begin{array}{ccccc}\chi_{1}^{2} & \chi_{\perp} & D_{\perp} & R_{p} & v_{\text {comv }} \\ {\left[10^{4} \mathrm{~cm}^{2} / \mathrm{s}\right]} & {\left[10^{4} \mathrm{~cm}^{2} / \mathrm{s}\right]} & {\left[10^{4} \mathrm{~cm}^{2} / \mathrm{s}\right]} & & {[\mathrm{m} / \mathrm{s}]} \\ 1.0 & \ldots & 0.25 & \ldots & 0 \\ 4.0 & \cdots & 0.50 & \ldots & 0 \\ 2.0^{*} D_{\perp}^{s} & 2.0^{*} D_{\perp}^{s} & 0.5^{*} D_{\perp}^{s} & \ldots & \ldots \\ 4.0 & 0.2 & 1.0 & 0.80 & 0 \\ 1.0 & 0.2 & 1.0 & 0.90 & -40 \\ 1.5 & 0.2 & 1.0 & 0.80 & 0 \\ 5.0 & 0.2 & 1 & 0.80 & 0 \\ 12.0 & 8.0 & 8.0 & 0.50 & 0\end{array}$

Stotier used a similar coupling technique ${ }^{3}$ of the b2 and DEGAS codes for the BPX study. The numerical procedure used to link the codes was similar to the procedure described above in the modeling of TFTR by Coster. In this simulation the divertor target was maintained orthogonal to poloidal flux surfaces. The system convergence criteria used in this simulation were the integrated ion plate flux, the peak plate heat flux, and the peak plate density. For the high recycling regime envisioned in BPX operation, a temporal mix of sources was necessary to avoid divergence in the plasm calculation, caused by the 
strong coupling of plasma and neutral parameters. Stotler used a mix of $30 \%$ previous iteration sources and $50 \%$ present iteration sources. The recycling coefficient value was 0.90 in the first iteration and slowly ramped to the expected operational value near unity in successive iterations. Unfortunately it was discovered after 30 b2/DEGAS iterations that convergence would probably be obtained after about 100 iterations for the BPX simulation, with each b2/DEGAS iteration consuming approximately 60 minutes of CrayII CPU time. Most of this CPU time was taken up by the Monte Carlo calculation in DEGAS. Hence the routine use of the b2/DEGAS coupling to model future devices must await improvements in the speed of the DEGAS code.

\subsection{Recent Advances in SOL modeling}

Many advances have been made recently in SOL modeling. These advances will not be discussed in detail but are mentioned here for completeness and for reference in the future work section.

A 2-D transient plasma transport model with a one-group diffusion approximation for the neutrals has been used 21 by Vold. Vold's code, EPIC, allows for a non-orthogonal geometry of magnetic flux surfaces and the target plates and ambipolar poloidal dritt. The code has been used to model CCT (UCLA), Textor and ALT-I, ASDEX, and ARIES-I (an ITER-like fusion reactor design). Comparison with the b2 code for the same case shows good agreement and the differences between the two codes can be explained by the different treatment of neutrals by the two codes. The research group at UCLA is designing a now codo 22 which uses finite volume elements in $a$ 2-D mesh that are fully trapezoidal in shape instead of the conventional rectangular shaped elements. The use of these general element shapes will be more accurate representation of angled targets; numerical approximations in this code are also more accurate than the ones used in present codes.

Recent efforts to improve upon the convergence problems of numerical techniques which are not completely implicit have led to the attempted solution of the fluid equations by the Newton-Raphson method. Knoll solved the fluid equations ${ }^{23}$ with the Nowton- 
Raphson method using an analytic Jacobian. Rognlien applied the Newton-Raphson solution technique to the governing equations of b2 with additional cross-field drifts included and created the LEDGE code24. Although both codes may be more robust than the b2 code in certain inatances because the solution schemes are almost fully implicit, the radius of convergence may be smaller for most problems and a good guess (preconditioner) is required to insure a high probability of convergence.

Finally impurities have almost been neglected in 2-D SOL modeling. The b2 code al!ows modeling of the tokamak SOL with high impurity fractions of helium, but only trace levels of carbon. Campbell developed a I-D impurity tranaport code25 that can handle up to $90 \%$ radiation fraction in the SOL, a value that is similar to the radiation fractions seen during carbon blooms 26 on TFTR. Successful implementation of selfconsistent carbon and higher-Z impurity radiation in 2-D plasma codes has not yet been reported. Accurate modeling of impurities is especially needed to simulate and spproximate the effectiveness of pumping systems in exhausting impurities and to model SOL shielding of impurity penetration into the core plasma. 


\section{CHAPTER 3}

\section{COMPUTATIONAL MODELS}

\subsection{Introduction}

The two primary codes used in this research are b2, 2-D multi-fluid edge plasma transport codel, and DEQAS, 3-D neutral transport code2. In the analysis presented here, toroidal aymmotry is assumed in the DIIl-D divertor calculation and cylindrical symmetry is assumed in the Tore Supra pump-limiter calculation to simplify the neutral transport calculation to two dimensions. Because both codes existed prior to the applications in this resaarch, each code will be described only briefly. However, the physics relevant to the coupling of the two codes will be discussed in detail, $\mathbf{a}$ will the special physics phenomena of intereat in this research. As stated previously, the primary focus of this research is on the plasma modeling side of the coupling and discussion of relovant plasma phyaics is weighted accordingly.

\subsection{The b2 fluid code model}

The general fluid equations used in the 62 model are derived from standard fluid equations 6 and are described eleewhere 7 , is is the solution technique. The equations presented here are simplificutions of the generd equations, using a single plasm ion species (hydrogen for Tore Supre, deuterium for DII-D) in the modal. The equations contain time derivatives, but it should be pointed out that time-stepping is employed only to allow under-rolaxution of the system of equations until a steady-state reault is obtained. The equations are colved only approximately during each time-step. The b2 code ofiors an internal single-species recycling model which contains problem-dependent parameters; this model will be discussed later. 


\subsection{Goveming Fluid Equations}

The plasma continuity equation for a single lon species and the assumption of quasi-neutrality, $n_{1}=n_{0}=n_{N}$, and $z_{\infty}=1.0$ is obtained from the standard equations used in the b2 model?:

$$
\frac{\partial n_{\mu}}{a}+\frac{1}{\sqrt{8}} \frac{\partial}{\partial x}\left(\frac{\sqrt{8}}{n} n_{\mu w_{2}}\right)+\frac{1}{\sqrt{8}} \frac{\partial}{\partial y}\left(\frac{\sqrt{8}}{n} n_{\mu w_{\nu}}\right)=S_{\mu}^{n}
$$

where

$n_{N}=$ planm density

$u_{x}=$ plama velocity in the x-direction (poloidal or toroidal direction)

$w_{y}$ - plaama velocity normal to the magnetic feld (radial direction)

$\varepsilon_{1} h_{x}, h_{y}=$ metric coefifcients

$s_{\mu}^{n}$ - plama ion panicle source term from plasma/neutral interactions

It is assumed that no current flows in the SOL, $20 y_{j}^{\prime}=u_{i}^{\prime}=u_{j}$. The momentum equation for the ions with the above assumptions is:

$$
\begin{aligned}
& \frac{\partial}{a}\left(m_{1} n_{N} \mu_{1}\right)+\frac{1}{\sqrt{8}} \frac{\partial}{\partial x}\left(\frac{\sqrt{8}}{b_{n}} m_{1} n_{\mu_{1}} n_{2} \mu_{1}-\frac{\sqrt{8}}{n_{n}^{2}} n_{1}^{1} \frac{\partial}{\partial x} \mu_{1}\right)+ \\
& \frac{1}{\sqrt{8}} \frac{\partial}{\partial y}\left(\frac{\sqrt{8}}{h_{y}} m_{1} n_{\mu} \mu_{2} \mu_{1}-\frac{\sqrt{8}}{h^{2}} n_{1}^{\prime} \frac{\partial}{\partial y} n_{1}\right)=\frac{B_{1}}{B_{m}} \frac{\partial}{\partial x}\left(p_{1}+p_{1}\right)+S_{m_{1}}^{\prime}
\end{aligned}
$$

where

$$
\begin{aligned}
& m \text { - ion mass } \\
& \text { y - plauma velocity parallel to the magnetic seld } \\
& H_{x}, H_{y}=x \text {-direction and transweree direction ion viscosity } \\
& B_{x}, B_{m}=x \text {-direction component and total magnetic Geld } \\
& p_{1}, P_{0}=\text { electron and ion thermal pressure }
\end{aligned}
$$

The second term in each derivative on the left-hand-side represents the viscous drag forces, and the first two terms on the right-hand-side represent the pressure gradient. The 
momentum source term is set to zero in all calculations. The equation of state relates pressure to density and temperature:

$$
p_{0}=n_{0} T_{a} ; a=\text { electrons,ions }
$$

The radial velocity is governed by a difmusion equation:

$$
w_{p}=-\frac{D_{1}}{n_{1}} \frac{\partial}{\partial y}\left(\ln \left(n_{N}\right)\right)-v_{m_{m}}
$$

where

$D_{1}=$ anomalous radial particle diffusion coefficient

Vom - anomalous inward radial convective pinch velocity

The electron energy equation takes the form:

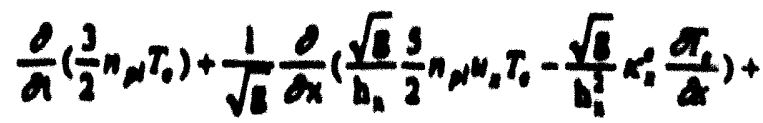

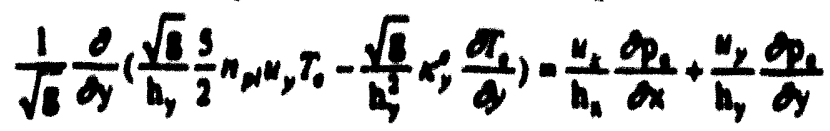

$$
\begin{aligned}
& -k_{\infty}\left(T_{0}-T_{1}\right)+S T
\end{aligned}
$$

where

$$
\begin{aligned}
& k_{1}, k_{2}=\text { dectron } x \text {-direction and radial direction thermal conductivity } \\
& k_{\infty}=\text { clastical equipartition coefreient }
\end{aligned}
$$

The firat term of the derivatives in the $x$-direction and the $y$-direction in the equation above represent electron convection, and the second term represents electron conduction. The arat two terms on the right-hund-aide represent work done by the electric field, the third term is equipartition of enerey between electrons and lons, and the last term represents the enercy source(aink) amociated with electron/neutral particle interactions and radiation loseses.

The ion energy equation has the form: 


$$
\begin{aligned}
& \frac{\partial}{a}\left(\frac{3}{2} n_{N} T_{1}+\frac{1}{2} m_{1} n_{N} w^{2}\right)+\frac{1}{\sqrt{2}} \frac{\partial}{d x}\left(\frac{\sqrt{2}}{h_{1}}\left(\frac{3}{2} n_{N} w_{1} T_{1}+\frac{1}{2} m_{1} n_{N} w_{2} w_{1}^{2}\right)\right.
\end{aligned}
$$

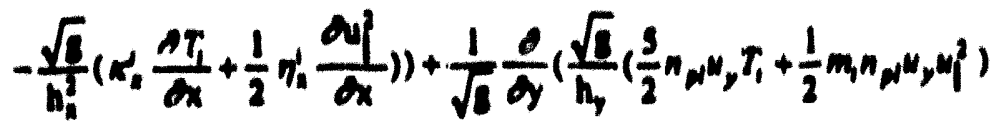

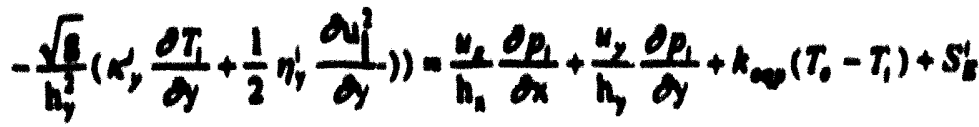

where

$$
R_{1}, R_{y}=\text { ion } x \text {-direction and radial direction thermal conductivity }
$$

The first and third terme of the derivatives in the $x$ and $y$ directions on the left-hand-side correapond to lon convection and conduction, analogous to the electron energy equation terms. The coconsl and fourth terms in the aforementioned derivatives represent kinetic enercy and viscous (metion) loses. The terms on the right-hund-alde of the equation are andogous to the dectron energy equations except that the equipartition term is added to the lons (aseuming electron temperature is higher than ion temperature for notation convention). The source term for ion energy comes from the average kinetic energy that Franck-Condon neutrals have (a fow oV) before they are ionized by electron impact. The cource terms are dependent on which recycling model is used in the b2 code and will be described in sections on the various b2 recyeling modds. The singlo-species recycling model in b2 does not include the enersy sink caused by charge exchange of cold noutrals with hotter plaems lons, but this sink is included when the b2 code is coupled to DEOAS because the source terms are obtuinad trom a DEOAS calculation.

\subsubsection{Transport Coefitidents}

The parallel transport coefficients used in the 62 modd' are clascical with scaling

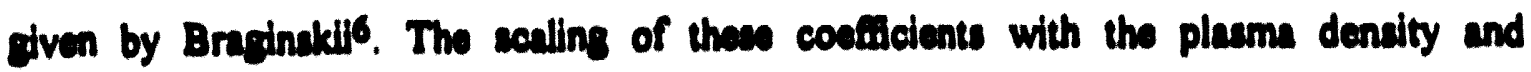
temperature is shown below:

$$
\text { of } \left.1 \mathrm{~cm}^{-1} \mathrm{~B}-1\right)=1.622 \times 10^{20} \mathrm{~T}_{0}^{2 / 2} \mathrm{loV}
$$




$$
\begin{aligned}
& \sigma_{1}\left|\mathrm{~cm}^{-1},^{-1}\right|=6.516 \times 10^{10} T_{1}^{3 / 2}|\mathrm{eV}| \mu^{1 / 2}, \mu=\frac{m_{1}}{m_{1}} \\
& \text { T }\left|8 \mathrm{~cm}^{-2} \mathrm{i}^{-1}\right|=2.67 \times 10^{4} T_{1}^{3 / 2} \mid \mathrm{OV} \mathrm{Lu}^{1 / 2}
\end{aligned}
$$

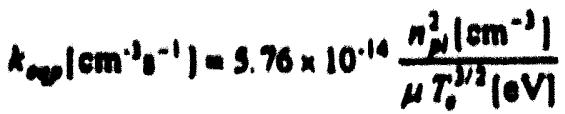

The $x$-direction components of the clasaical parallal electron and lon thermal conductivity are given by:

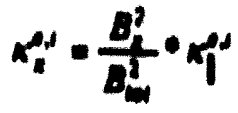

The ame relation is used to obtain $\eta_{a}$.

Athough particle fow along feld lines is more rapid than cross-fidd fow, the parallel connection length is alco much longer. Hence, both parallel iransport and radial transport are relevent for particle flows. The dominant momentum flow mechanism was not inveatigated thoroughly, but it is expected that the parallel momentum flow transport dominates the other terms. It should be noted, however, that the parallel viscosity momentum loss term can numerically dominate the momentum balance in regions where the parallel flow derivative is large, l.e. near neutralizer plates. Consequently, a kinoticbased frow-streaming limit on parallal viscosity is recommended in all future simulations (cee the next section). Becaure of the strong dependence of parallal thermal conductivity on the plasma temperature, parallal conduction dominates the enercy transport in most SOL regimes above $10 \mathrm{oV}$. Moreover the parallal dectron conduction term tends to dominate the total energy bulance because the dectron themal conductivity is greater than the ion thermal conductivity by roughly the equare root of the mases, $\sqrt{m_{1} / m_{0}}$. In very high denaity and low tempernture regimes (below a fow oV), equipartition dominates the enerey balanas, efrectivaly constraining electron and ion temporature to be equal. Ion convection near the plate is often a signifeant term in the overall energy balance. 


\subsubsection{Free-Streaming Limits on Conduction and Momentum}

Because the average plasma fluid velocity must be sub-sonic everywhere in the SOL upatream of the sheath (see chapter I for discussion of physics), the conduction electron and ion heat flux should not exceed the flux that would be carried to the target if all of the SOL particles were traveling at their thermal speed. Numerically, however, the eloctron and ion heat flux can fur exceed the aforementioned value, referred to as a convective free-streaming-limit. Rognlien estimated through kinetic simulations 27,28 that the electron and ion conduction heat flux should be limited to a fraction of the freeatreaming-limit. This fractional multiplier was arrived at by numerically computing heat flux for a test case and comparing that value to the heat flux calculated by the SpitzerHarm formula. Rognlien computed the value of the conduction flux limit factor, $a^{\prime}$, , to be between 0.1 and 0.2 for both lons and electrons. In the b2 code, the free-atreaminglimit on conduction is applied by means of an effective thermal conductivity, given by:

$$
x_{2}=k_{2}^{2}\left(1+\left|\frac{q^{k}}{q h}\right|\right)^{-1}, \text { anelectrons,ions }
$$

where

$$
q_{:}^{0}=-x_{*} \frac{\partial T^{0}}{\partial x} \text { (classical Spitzor-Harm heat flux) }
$$

and

$$
q R_{2}=a h_{m} n_{\alpha} k_{b} T_{a} \sqrt{k_{b} T_{0} / m_{0}}
$$

The dux-limiting of the electron conduction term is more important because this term dominates the energy balunce, and most present day 2.D SOL analysis codes employ such a limit. Werley discovered' that flux-limiting of the ion conduction term yielded significant difierences in the plama urget parameters when compared to the calculations in which a flux limit was not applied. Comparicons of plasma simulations with and without the ion conduction flux limit for the DII-D simulation will be diccussed in Chapter 4.

Using the same physical argument 25 in the previous paragraph, the ion momentum tux should not exceed the amount of momentum flux that could be carried if all ions were 
traveling at the ion sound speed. A momentum flux limit can be applied as an effective viscosity coefficient, similar to the calculation of an effective thermal conductivity:

$$
\eta_{10}=\eta_{1}^{0}\left(1+\left|\frac{\Gamma_{1}}{r_{L}}\right|^{-1}\right.
$$

where

$$
\begin{aligned}
& r_{a}=-\nabla_{1}^{\prime} \frac{\partial T_{1}}{\partial x} \\
& r_{R_{2}}^{\prime}=a_{n_{L}}^{\prime} \frac{B_{L_{m}} n_{m} k_{b}\left(T_{c}+T_{1}\right)}{B_{m}}
\end{aligned}
$$

Unfortunately no kinetic predictions of the value of a'nu were available at the time of this research. A value of 1 was used for the viscosity flux limit factor in the Tore Supra enalysis, as will be discussed in the next chapter.

\subsubsection{Boundary Conditions}

The boundary conditions used to solve the set of governing equations for b2 are geometry-dependent, of course. Alone a stagnation (symmetry) boundary normal to the $x$-direction, zero parallel density gradient, zero momentum flux, and zaro parallel temperature gradient are usually imposed. This condition is applied along edge CD of fig. 4 of the Tore Supr modeled region. No stagnation boundary is assumed for the DIII-D simulation.

Along the sheath interface in front of a materid boundary normal to the $x$ direction, zero parallel density eradient is applied and parallel ion velocity is equal to the ion sound (thermal) speed. Also, energy trunsmission fectors are speciffed such that the electron and ion plate heat fluxes are limited in constant factors times the plate particle กlux:

$$
q_{A}=\gamma_{0} m_{0} v_{a} k_{b} T_{0}, a=e l e c t r o n s, \text { ions }
$$

where $\gamma$ is the energy trensmission factor and $v_{w}$ is the particle thermal velocity. Although $\gamma$ is related to the secondary electron emiasion coefficient 29 , constants are used in the b2 code to simplify the problem: $\gamma_{0}=4.0$ and $\gamma_{1}=2.5$. The kinetic energy component of the 
ion heat flux is computed separately and not included in the ion energy transmission factor. The magnitude of the kinetic energy component increases the numerical value of the total energy transmission factor by about 1, resulting in a total (electron plus ion) energy transmission factor of about 7.5. These conditions are applied at the neutralizer plate for Tore Supra (edge $A B$ of fig. 4) and at both divertor plates for the DIII-D analysis.

Along the inner boundary normal to the y-direction, density and temperature are specified, as well as no shear (zero gradient in the radial direction) in the parallel velocity. This condition is applied along the edge $\mathrm{AD}$ of fig. 4 for Tore Supra (not including the region under the limiter head) and also along the inner core boundary for DIII-D. The boundary under the limiter head in the Tore Supra pump-limiter throat is a material boundary and discussed in the next paragraph. Another applicable and relevant boundary condition along the inner boundary is the specification of the total power crossing the inner boundary. This boundary condition sets the ion and electron temperature gradients along the inner boundary instead of fixing the temperatures themselves. However application of the boundary condition directly can result in variations of plasma temperature along the inner boundary closed-flux-surface. Hence, the preferred method for application of this boundary condition is adjustment of the temperatures along the inner boundary until the total power crossing the separatrix into the SOL matches the desired power input level30. This method entails several runs the code.

Along the wall boundary normal to the y-direction, several conditions may apply. Zero plasma density gradient or the use of an extrapolated endpoint (gradient-scalelength) can be used as the boundary condition for the continuity equation. Zero plasma temperature gradient, a finite temperature gradient-scale-length, or pedestal temperatures can be used as the boundary condition for the energy equations. The last of the three temperature boundary conditions listed above is sometimes necessary to avoid collapse of outer boundary temperatures, which are sometimes irrelevant to calculations of such 


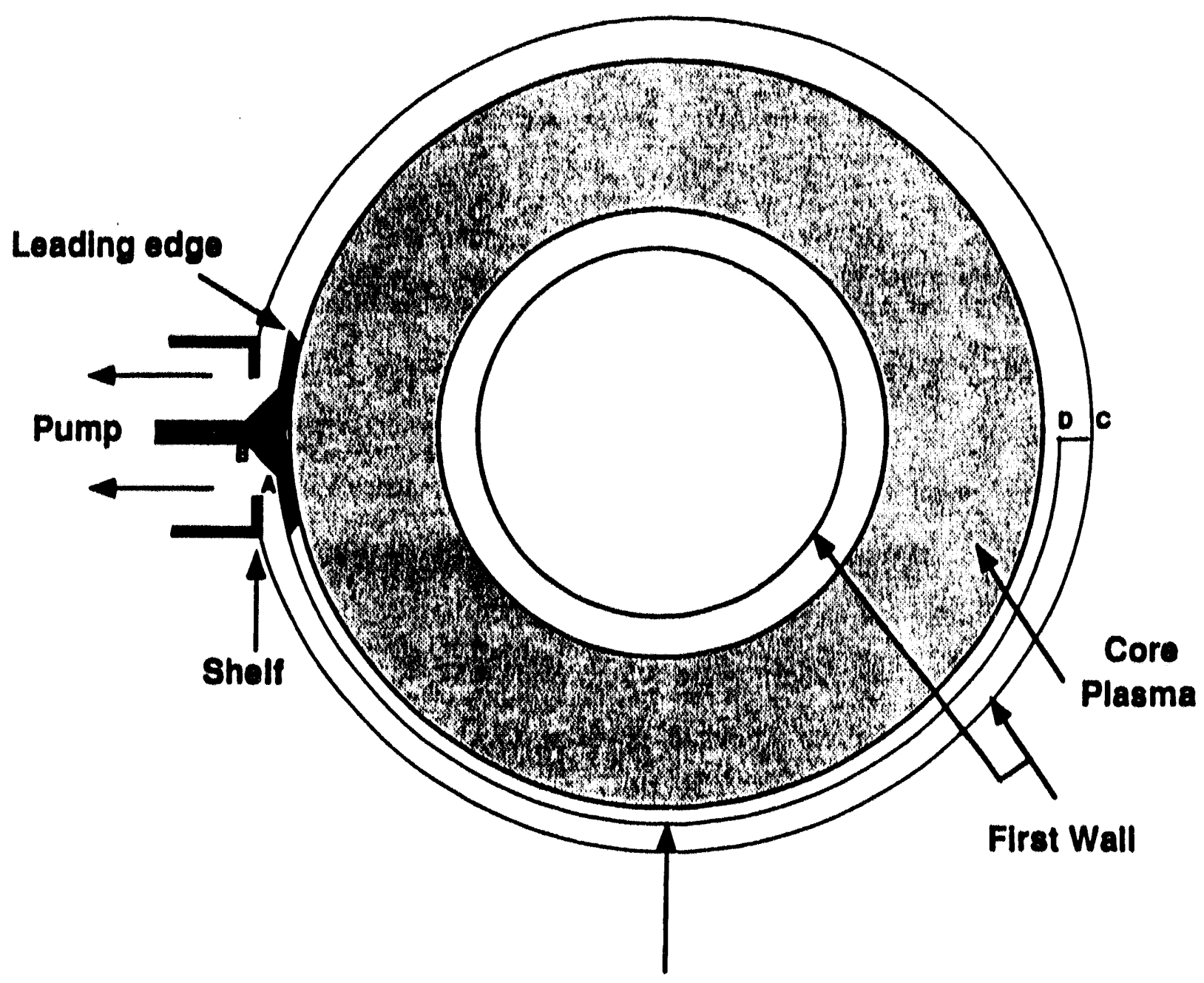

Scrapo-off-Layer Plasma

Figure 4: Toroidal view schematic of Tore Supra SOL end outboard pump-limiter

quantities as target plate peak heat flux. On the other hand, use of the pedestal temperature boundary condition may allow a steady-state solution which does not physically exist. Zero shear in the parallel velocity is applied as the momentum equation boundary condition at the outer wall boundary. For the Tore Supra simulation, gradientscale-lengths for density and energy are specified (edge BC of fig. 4). Under the limiter 
head in the Tore Supra pump-limiter throat (small part of edge $A D$ of fig. 4), zero density and temperature gradients are specified.

Several different boundary conditions were tried along the wall and underside of the limiter head for the Tore Supra analysis. Only one set of boundary conditions was used for the DIII-D analysis, the same ones used in the 1990 and 1991 benchmarks.

\subsubsection{Internal One-Species Recycling Model}

As previously mentioned the $b 2$ code offers a simple single-species recycling model. A new recycling model which uses source terms calculated by the DEGAS code was written and implemented to allow the external coupling of b2 and DEGAS. Fixing the free parameters in the b2 single-species recycling model to match coupled b2/DEGAS results required scaling of the free parameters with input power and assumed radial transport coefficients. Therefore the single-species model was abandoned in favor of a new two-species molecular physics model, the core of which was created by Hogan 31 . The internal single-species model is described below, the recycling model using DEGAS source terms is described in the b2/DEGAS coupling section, and the new two-species molecular physics recycling model is described in a later section.

The b2 code offers a simple single-species (atomic) neutral recycling model. In this model, the first generation neutral flux off of the plate is followed until it is completely ionized. Charge exchange cannot be modeled because multiple generations of neutrals are not tracked. The fraction of the incident ion flux at the neutralizer plate, $\Gamma_{\text {plese }}^{\prime}$, being reemitted as neutrals, $\Gamma_{p \text { mon }}^{0}$ is given by a constant recycling coefficient, $R_{p}$ :

$$
\Gamma_{\text {min }}^{0}=R_{p} * \Gamma_{\text {man }}^{\prime} \text { (part./s) }
$$

All neutrals are assumed to come off the neutralizer plate as atoms. These neutrals have an average energy in the $x$-direction (enex) and radial direction (enerad), both taken to be $5 \mathrm{eV}$ in this model. The default values for enex and enerad imply a neutral temperature of $10 \mathrm{eV}$, which is meant to be a rough average of the molecules desorbed at the walltemperature $(<0.5 \mathrm{eV})$ and the fast, reflected atoms $(>50 \mathrm{eV})$. In general, most of the ions 
striking the neutralizer plate recycle as desorbed molecules. In this manner the physical process of two-species neutral transport (desorbed molecules and energetic reflected atoms) is approximated as single-species transport with an average energy. The singlespecies neutral thermal velocity is given by :

$$
v_{t h}=\sqrt{\frac{2 * k_{b} * E_{0}}{m_{i}}}(\mathrm{~m} / \mathrm{s})
$$

where

$$
\begin{aligned}
& E_{0}=\text { neutral thermal energy in eV (enerad or enex) } \\
& m_{1}=\text { ion mass in } \mathrm{kg}
\end{aligned}
$$

The neutrals are first transported up the same flux tube in which the incident ions came to the plate. The deposition in the first cell adjacent to the plate in the $x$-direction is given by the minimum of $a^{*} \Gamma_{\text {mim }}^{0}$ and the ionization reaction rate:

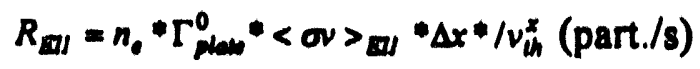

where

$$
\Delta x=\text { length of cell in the } x \text {-direction }
$$

The factor $a$ in the term $a^{*} \Gamma_{\text {ane }}^{0}$ is equal to 0.5 in the cell adjacent to the plate and 1.0 in all other cells; it represents a numerical limit imposed to prevent all of the ionizations from occurring in the cell adjacent to the plate. All other terms have been defined previously. The number of neutrals deposited in the first cell is subtracted from the total number of available neutrals, $\Gamma^{0}$. Next all of the remaining neutrals are transported into the next adjacent cell away from the plate in the x-direction. The number of neutrals deposited in the second cell is again given by the above equation, and the remaining available neutral flux is reduced by the number deposited in the cell. This process is repeated until all of the neutrals have been deposited, or the neutrals reach an imposed limit on the distance they can travel upstream of the plate. The default limit is the parallel flow stagnation point, and neutral flux left over in the last cell is discarded. In a calculation with one plate, the neutrals are allowed to travel all the way to the other boundary, a stagnation boundary. In 
a full two-plate calculation (i.e. the DIII-D simulation), the neutrals are allowed to go halfway between the plates. Neutral flux in the private-flux-region is allowed to be transported to the (imaginary) boundary between the inboard and outboard sides of the private-flux-region. The $x$-direction neutral transport calculation is carried out for each flux tube striking the plate, beginning with the innermost radial flux tube. The distribution of particles in the $x$-direction is equivalent to distribution along a mean-free-path given by:

$$
\lambda_{x}=\frac{v_{t h}}{\left.n_{0}^{*}<0 v\right\rangle_{a l l}}
$$

Next the neutrals are transported in the radial direction, which leads to the eventual 2-D ionization distribution. Starting from the innermost radial cell along the plate, a fraction of the neutrals deposited by the $x$-direction transport is transported radially outward (frout). Usually the neutralizer plate is normal to the $x$-direction; in this case, the recycled neutral flux is expected to have an isotropic distribution. Hence, frout is assigned the value of 0.5 , which means half of the recycled neutral flux travels radially outward and the other half travels radially inward. The radial thermal velocity is given above. The ionization source rate in the innermost radial cell along the plate is given by the minimum of the available neutral flux, $\Gamma_{y}^{1}$, and the source rate calculation:

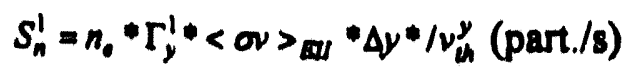

where

$$
\begin{aligned}
& \Delta y=\text { length of cell in the } y \text {-direction } \\
& \Gamma_{y}^{\prime}=\Gamma_{x}^{\prime} \text { *frout } \\
& \Gamma_{y}^{\prime}=\text { ionization deposition in cell } 1 \text { from } x \text {-direction transport }
\end{aligned}
$$

The number of ionizations occurring in the first cell is now subtracted from the available flux, and the remaining available flux is transported to the next cell radially adjacent to the innermost cell. Next the ionization deposition in the second cell from the $x$-direction calculation is added to the available flux, and the relationship between the available neutral flux in cell 2 to the available neutral flux in cell 1 is given by: 


$$
\Gamma_{y}^{2}=\Gamma_{y}^{1}-S_{n}^{1}+\operatorname{srour} * \Gamma_{z}^{2}
$$

The calculation progresses radially outward until the outer wall is reached. Then the remaining neutral flux is swept radially inward, and the number of neutrals remaining from the $x$-direction sweep are added to the total available neutral flux. For example, the neutral flux in the last (nth) radial cell, the first one in the inward sweep calculation, available to be transported to the $(n-1)$ cell is related to the neutral flux at the ond of the first outward radial sweep by:

$$
\Gamma_{y, \mathrm{~N} n}^{n}=\Gamma_{y, \text { out }}^{n}-S_{n}^{n}+(1.0-\text { frout }) * \Gamma_{z}^{n}+\Gamma_{\text {mall }}
$$

where $\Gamma_{\text {mall }}$ is the ion flux recycling off the wall. This sweep continues inward until the innermost radial cell along the plate is reached. The ionization in each cell is based on (3.23). At the end of this irward sweep, all of available neutral flux is usually not ionized. Hence, if there is available neutral flux left over from the previous sweep, this flux is swept radially outward again, with the additional ionization rate given by (3.23). This sweep differs ttom the first outward sweep because all of the neutral flux from the $x$-direction sweep has been added to the available neutral flux during the first two sweeps and no flux is left to be added in during this sweep. This third sweep progresses radially outurard from the innermost radial cell to the outermost cell as long as the neutral flux left over from the first two sweeps is greater than 0. This set of radial sweeps is performed for each set of radial cells, beginning with the ones on the plate and progressing upstream. Next the integrated ionization source is normalized, in case any neutral flux remains after the $x$ direction sweep or the three $y$-direction sweeps:

$$
\int s_{n}=R_{p} \cdot \int r_{\text {m }}^{\prime}
$$

This simple single-generation neutral tracking model allows neutral flux to transport across flux tubes in the radial direction.

Next the electron and ion energy source terms are computed. The amount of energy needed to ionize the neutral atoms is deducted from the local electron fluid where ionization occurs at a fixed rate of $25 \mathrm{\circ V}$ per ionization (eloss), which represents 
ionization and excitation losses. The local ion fluid gains energy at a fixed rate of $5 \mathrm{eV}$ per ionization (Igain) which represents the approximate average kinetic energy of the FranckCondon-like neutrals emitted from the target directly or bom after dissociation of desorbed molecular hydrogen. This model computes average recycling sources fairly well. However DEGAS calculations show that eloss can be much higher than $25 \mathrm{eV}$ in the outer region of the SOL near the wall, and igain can be less than zero when the charge exchange loss mechanism is taken into account. Hence, as stated previously, the five ftee parameters, eloss, igain, frout, enex, and enerad, can be adjusted to match coupled b2/DEGAS predictions of ionization sources. Unfortunately the above parameters may require scaling with input power and transport coefficients to obtain a good match.

\subsection{The DEGAS Monte Carlo Calculation}

The DEGAS code computes neutral transport via the Monte Carlo technique in up to three directions ${ }^{32}$. In this analysis, only two dimensions are used with the assumption of symmetry in the third direction. The neutral/plasma interactions modeled by the code for hydrogen are reproduced here from Heifitz 2 :

$$
\begin{aligned}
& H^{0}+H^{+} \rightarrow H^{+}+H^{0} \\
& e+H^{0} \rightarrow H^{+}+2 e \\
& e+H_{2}^{0} \rightarrow 2 H^{0}+C \\
& e+H_{2}^{0} \rightarrow H^{0}+H^{+}+2 e \\
& e+H_{2}^{0} \rightarrow H_{2}^{+}+2 e \\
& e+H_{2}^{+} \rightarrow 2 H^{0} \\
& e+H_{2}^{+} \rightarrow H^{0}+H^{+}+\bullet
\end{aligned}
$$

The reactions liated above are hydrogen charge exchange, electron impact ionization, disecociation, dissociative ionization, molecular ionization, dissociative recombination, and dissociation. The dependence of the cross-sections on electron temperature is shown in 
(Gg. 5). If hydrogen molecular ionization occurs, it is assumed that the molecular ion is dissociated immediately by dissociative recombination or dissociation. Details on the specific Monte Carlo techniques, the wall redection models. the track-length estimator, and other information about the DEGAS algorithms is given elsewhere 32 and will not be discussed here.

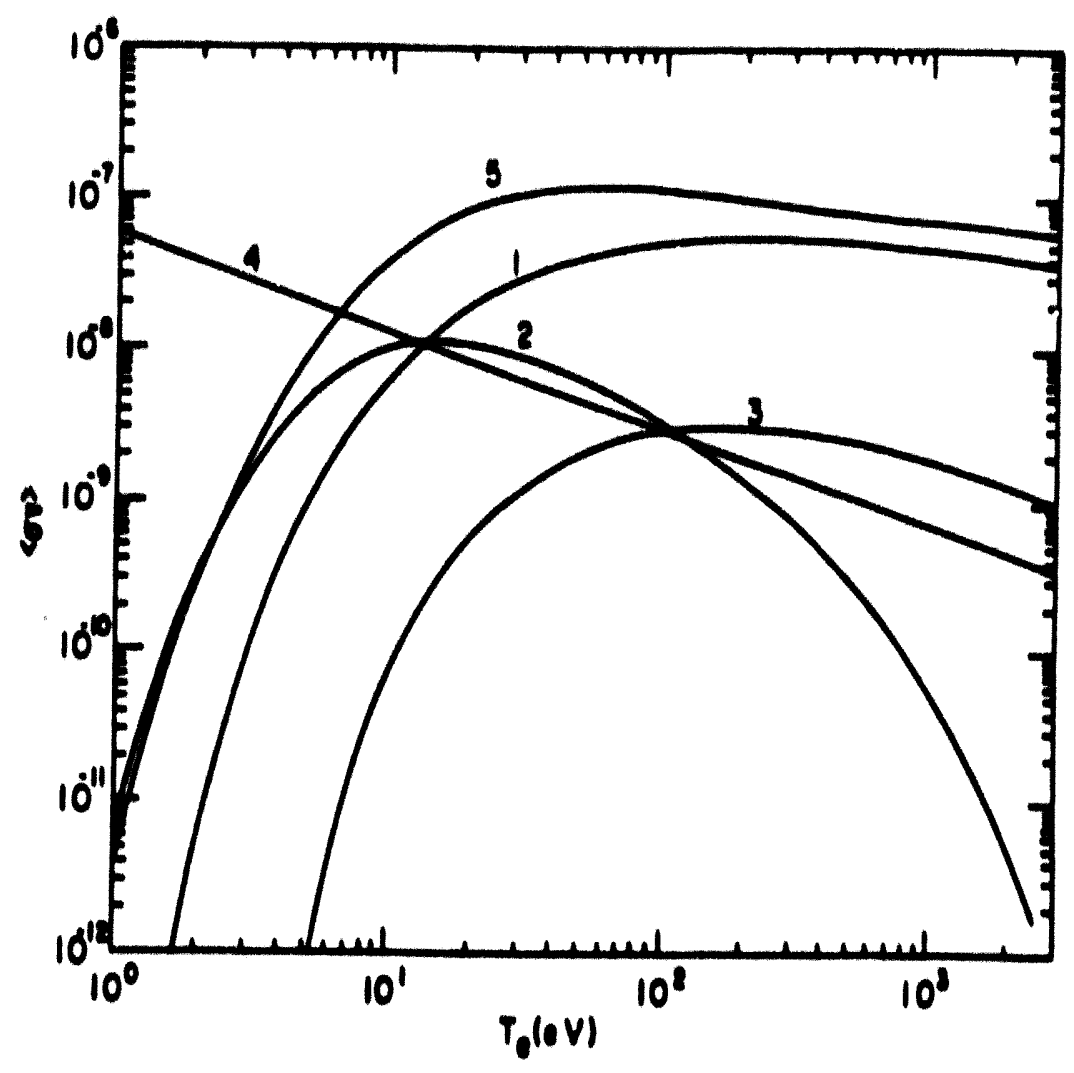

Figure 5: Resction rate coefficients for the following procesces32:

(1) : $\bullet+\mathrm{H}_{2}^{0} \rightarrow \mathrm{H}_{2}^{*}+20$ (eqn. 3.31)

(2) : $\bullet+\mathrm{H}_{2}^{0} \rightarrow 2 \mathrm{H}^{\circ}+\mathrm{O}(\operatorname{eqn} .3 .29)$

(3) : $\bullet+\mathrm{H}_{2}^{0} \rightarrow \mathrm{H}^{0}+\mathrm{H}^{+}+2 e($ (eqn. 3.30)

(4) : $\bullet+\mathrm{H}_{2}^{*} \rightarrow 2 \mathrm{H}^{\circ}$ (eqn. 3.31a)

(5) : $\bullet+\mathrm{H}_{2}^{*} \rightarrow \mathrm{H}^{0}+\mathrm{H}^{+}+\boldsymbol{e}(\operatorname{eqn} .3 .3 \mathrm{Ib})$ 


\subsection{Coupling Between b2 and DEGAS}

The procedure used in this analysis to couple the b2 and DEGAS codes begins with a run of the b2 code, using its internal single-species recycling model. Calculations of the plasma parameters are written to an input file for the DEGAS code, which then computes the recycling sources. These sources are utilized in the next run of the b2 code snd the codes are run altermatively until convergence of system diagnostics is achieved. The procedure is described below.

\subsubsection{Information Transfer and 2-D Grid}

The 62 code provides information on the average plauma density, electron temperature, and ion temperature in the volumetric mesh zones considered in the DEGAS calculation. The b2 calculation of ion plate flux is written into the DEGAS input file, as well as information on plasma momentum in both directions if required. This information is not used in the DEOAS calculation for this analysis. The DEOAS calculation provides electron enersy, lon energy, and particle cource terms associated with the recycling and radiation procesces modeled by the code (linted above). The DEGAS calculation of the Tore Supra pump-limiter also extimates the pumping efficiency, $n_{\text {monn }}$, which is the faction of ions that atrike the plate and are pumped. The pumping efticiency is related to the effective recycling coefficient by:

$$
R_{i}^{*}=1-\eta_{m}
$$

This effective reoycling coeticient is used in the b2 calculations for Tore Supra to simulate pumping. The pumping or removal efficiency mentioned above is a difierent parameter than the commonly quoted exhaun etweiency of the pump-limiter, which refers to the taction of the core eftlux that is removed by the pump. It should be noted that the DEOAS calculation removes particlas as the actual pump location, whereas the b2 calculation removes particles adjecent to the pump location at the target plate.

The easiear way to transfer information ffom one code to the other entails the use of the sume 2-D grid in both codes. One mun be careful in selecting the grid cells near the 
cell center. After the DEOAS calculation, the recycling source terms are initially available only on the coarse DEOAS grid. The particle and electron energy source terms are computed as follows: the radial ecale length $\left(\lambda_{\infty}\right)$ between each pair of radially adjoining cells is computed at each toroidal location. The source terma in a particular b2 cell are eatimated uaing the scale length computed in the previous calculation:

$$
s_{n, \infty}^{2}=s_{n, \infty}^{\infty} \exp \left(-\frac{n_{2}-r_{m a}}{\lambda_{\infty}}\right)
$$

The source term calculation is repeated for all cells at a given radius, and the entire interpolation procedure is repeated for the toroidal direction. The only difference for the extimation of the ion enercy source terms on the 62 mesh is that linear interpolation between cells, not exponential interpolation, is used. Linear interpolation is necescary for the ion energy sources because they are not emooth, i.e. they are greater than zero in regions of high particle source and less than zero in regions of high charge exchange.

The DEGAS calculations for the Tore Supre dimulation differ from the DII-D simulation in that particles are allowed to escape from the computational domain if they exit the pump-limiter throat. This provision is required to reduce the calculation time because neutrals that are transported out of the pump-limiter throat have a long ionization mean-free-path. Hence, tracking of these neutrals requires significantly more CPU time than neutrals ionized in the throat. The escaping fraction of recycled niutrals, is defined as the fraction of the incident plate neutral lux that exits the chroat prior to ionization, and this quantity is computed by the DEGAS code. In the b2 part of the b2/DEGAS nun, therefore, the eccape trection of the neutral Aux is ionized outhide of the throat by the following procedure. The eacaping neutral flux in ach olux tube is diatributed along cells in each alux tube outside of the throat with an asoumed lonization mean-free-path of $10 \mathrm{~cm}$. No neutral transport across flux tubes is allowed outside of the throat. Next, the computed volumetric neutral ionization rate in the coll adjecent to the pump-limiter throat entrance (the "outaide cell") is compared to the ionization cource rate 
in the adjoining cell inside the throat (the "inaide call") within a given flux tube. If the compured source rate in the "outside cell" is greater than the "inside cell" for a particular flux tube, the ionization rates in all cells outside of the throat are scaled by the ratio of the lonization fates of the "inalde cull" and the "outside cell". Hence a umall portion of the escaping neutral flux from the DEGAS calculation is allowed to eacape from the b2 SOL as well. This allowed loss of neutral particles is necessary to maintain a smooth toroidal profile of recyeling source terms in the Tore Supra simulation. The (comewhat arbitrary) technique used to handle the (DEOAS) neutral flux that escapes out of the pump-limiter throat actually has an inaignificant impact on the computed SOL parameters outside of the throat (B2). No such provision to allow particle escape routes is needed in the DIII-D simulations because of the higher SOL plasma density, which reduces the neutral meanAro-path.

One final concern when fine grids are used both in the b2 and DECAS codes is the calculation of cell volumes, which are computed difierently in each code. Different grid volumes can lead to incorrect source term diatributions used in the b2 code. The b2 code computes the volumes numerisally based on the input cell boundaries. The DEGAS code, however, places the input grid onto a reference mesh and computes the grid volumes based on the number of reference cells in each plasma grid cell. The DIII-D simulations were initially done with the default reference mesh, but the results from the calculations indicated that the defult reference mech was too coures to compute plauma grid volumes properly. Hence the DIII-D simulations were repeated with a reference mesh with roughly sixtcen times the number of cells $2 \mathrm{~s}$ in the course reference meat. One b2/DEGAS aimulation was done with a reference mech contuining four times the cells in the coarse mosh and this calculation appeared to yidd accoptable results. The now referenice mosh increased the CPU time requirement for each calculation by 1.5 minutes on a fourprocessor Cray-II, independent of the number of neutral nights tracked. This problem occurs only when the DEGAS grid near the plate is tine and is not an issue with the Tore 
Supra simulation. In any ovent, it should be noted that the volumes calculated in the two codes can diffor by $3.10 \%$, even for the aume grid, due 10 practical limitations of the reference meah used in the DEOAS calculation.

\subsubsection{The Recyeling Model Uaing DEOAS Source Terms}

\subsubsection{Basic Description}

The DEOAS code computes all volumetric particle and energy source terms, which are then read in to the $b 2$ code. These volumetric source terms are converted to total source terms we described in the next section and used in the fluid equations to compute now thermal equillbrium. An option in this recycling modal allows for normalization of the DEOAS source terms with the integrated ion plate flux multiplied by the reoycling coefficient, as diven in (3.26), during each b2 intermal iteration. For the SN DII-D divertor aimulations, the recyeling source terms are normalized separately on the inbourd and outbourd sides. Sealing of cource terms typically allows the 62/DEOAS coupled caloulation to take laree ateps toward an equilibrium, but if is cometimes undeairable close to an equillibrium as the correction can progress too quickly. In weh cases where the radius of convergence of the problem appears to be umall, i.e. when small steps toward an equilibrium solution are numerically necescary, fixing the source terms during each b2 internal iteration achieves convergence.

\subsubsection{Mesh-oriented Considenations}

Several problems with the use of DEOAS source torms within the b2 code can aries if the volumes computed by the two codes for the sume meah are significantly difierent. The following procodure is uned to eliminate any source term profile differences that might occur because of difierent meahes in the two codes:

1. The DEGAS volumetric cource terms for each cell are multiplied by the correuponding DEOAS cell volume for each cell. This step maintains the profiles computed by the DECAS code. 
2. Each source term sbove is multiplied by the ratio of the total DEGAS cell volume divided by the total b2 cell volume. This step ensures that the integrated source terms in both codes have identical magnitudes.

The DIII-D resulte reported in Chapter 4 from the b2/DEGAS simulations were computed with the above technique. The b2 simulations for Tore Supra used the DEGAS volumetric source terms mulitplied by the b2 cell volumes, which resulted in a difference between the integrated source terms used in the b2 code and the integrated source terms computed by the DEGAS code. The difierence is acceptably small $(<2 \%)$ because the DEGAS grid cells are large enough such that the volume computation between the two codes yields very similar results. Future simulations of the Tore Supra tokamak, however, should include an appropriate normalization procedure to eliminate this small error.

\subsubsection{Statistical Error and Numerical Limits}

The DEGAS cource terms have a Monte Carlo statistical error associated with them. The find iteration of the DEGAS code in the b2/DEGAS calculation is done with enough teat flights to insure that the statiotical error associated with the peak source terms (usually at or near the separatrix on the plate) is less than $\mathbf{5 \%}$. However, the statistical error of the electron energy source terms in the outer part of the SOL near the wall and in the private-flux region can be $50 \%$ or higher. These cells fur away from the energy source of the core plaama cannot suatuin large ionization electron enersy sinks without collapsing thermally, and the Monte Carlo statiotical error often allows large uncertainties in the sink terms precisaly in these regions. The charge exchange energy sink for ions is also subject to large statistical uncertainties. Therefore it is occasionally necessary to enforce a limit in the magnitudes of any or all three of these source terms. The limits are enforced as fractional reductions of the cource magnitudes computed by the DEGAS code. The private-alux-region and outer SOL reductions are enforced gradually wuch that the colls furtheat away from the ceparatrix are reduced the moat. The charge exchange reduction is enforced anywhere that the total ion enercy source rate is leas than 0 , with the reduction 
being uniform for all cell source terms that qualify. Another reduction insures that the total electron energy ionization sink (per ionization occurring) is less than a prescribed limit, unually 100 ev/ionization. The last limit is used because the DEGAS code predicts a very high value for the evfionization in low temperature regimes. This would eventually lead to a numerical thermal collapse in the calculation which may not physically occur because the plasma would not suatain the lonizations necessary to generate such a large sink term. After all of these limits are applied (if needed), a global power balance ensures that the integrated energy source terms for eloctrons and ions matches the DEGAS calculations. This entire procedure leads only to a minor distortion of the energy source profiles, with the largeat distortion in cells far away ftom the regions of intense recycling at the coparatrix.

One further limit which is occasionally needed is a fractional reduction of all electron energy source terms on the inbourd side of the single-null divertor simulations. This limit prevents thermal collapse, but results in a reduction of the integrated DEGAS source terms on the inboard side. Such a limit is not needed on the outboard side because more power is fed into the outboard side due to the larger source region. This reduction of the integrated electron energy eource term is justified because of the uncertuinties in the DEGAS atomic physics daten which is discussed in the last chapter. It should be noted that the aforementioned limits are primarily needed in the firat fow coupled b2/DEGAS runs, and that these limits are generally removed as the b2/DEGAS calculations approach convergence. In some regimes, however, the use of these limits may allow a numerical solution where no physical solution actually exiats, and this possibility should be investigated further in the future.

\subsubsection{Advantages and Diandvantages of b2 Recycling with DEGAS}

Dieadvantages and advantages of the DEGAS recycling model as compared to the b2 singlo-species internal model are now discussed. The singlo-species recyeling model prevents thermal collapse of the electron fluid resulting from very high recycling sources 
because these sources decrease in magnitude as the electron temperature goes down due to sharply decreasing $\langle 0 v\rangle_{\mathrm{g} /}$ with decreasing electron ten perature (below $10 \mathrm{eV}$ ). This safeguard is not present in the b2 recycling model using DEGAS source terms because the source terms are held approximately fixed during the b2 calculation, and the lack of a feedback system between the plasma and neutrals is the major disadvantage of this model. Of course, the major advantage of using the DEGAS source terms is the detailed molecular and atomic physics in DEGAS which are not included in b2. The need for numerical limits which are problem specific is, of course, a significant disadvantage.

\subsubsection{Numerical Coupling and Iteration Procedure}

As stated previously, the first iteration of a simulation entails the use of the b2 code with its internal recycling model. The b2 code is an iterative code itself, and the code is run to convergence, which usually takes betweeni 1 and 3 CPU minutes on a CRAY-II. The next loop entails running the DEGAS code with the b2 plasma information, transferring source term data to the $b 2$ code, and running the $b 2$ code until convergence again. In the Tore Supra simulation, 2400 test flights are used per DEGAS simulation. The DEGAS/b2 loop is repeated until convergence of system parameters is achieved. The DEGAS calculations take about 60 CRAY-II CPU minutes for the pump-limiter simulation.

The DIII-D simulation is done slightly differently. To conserve CPU time, the first DEGAS run consists of 2500 test flights, as does the second run. The third and fourth DEGAS runs use 5000 test flights, and the rest of the DEGAS runs use 10,000 test flights until convergence is achieved. The CRAY-II CPU requirement per 10,000 test flights for the DIII-D divertor simulation is about 20 minutes. Source-scaling within a b2 iteration takes larger steps toward systematic convergence, but can cause system oscillation near convergence as the radius of convergence is often small. Once the system approaches convergence, therefore, the scaling of DEGAS sources within each b2 iteration is stopped. Fixing sources within a b2 run insures smaller changes in the computed plasma parameters 
from one b2/DEGAS iteration to the next. Hence this option is used only when the computed SOL parameters are believed to be close to a (computed) equilibrium.

\subsubsection{System Convergence Diagnostics}

For the Tore Supra pump-limiter simulation, $\eta_{\text {rom }}$ affects the flux amplification factor and plasma parameters, and is therefore used as a system convergence diagnostic. Convergence of peak plasma parameters is assumed to occur when the peak values change by less than 5\%, the order of the DEGAS statistical error at the peak values. Convergence of plasma profiles is more arbitrary with no definite limits set. The values of the integrated and peak ion plate fluxes in b2 affect the DEGAS calculation, so both of these quantities are used as diagnostics. The magnitudes of the peak DEGAS source terms, especially particle and electron energy, and the average radial and axial falloff lengths of the source terms are also monitored. The ion energy source term plays a less critical role in energy transport of the simulations as the magnitude is much smaller than the electron source term.

The DIII-D divertor simulation does not compute a recycling coefficient, so this parameter can not be used a convergence diagnostic. The other system diagnostics mentioned above for the Tore Supra simulation apply here also. In addition, because the plate heat and particle flux is directly related to the plate plasma density and temperature, the peak values of these parameters are monitored for convergence. Convergence of these parameters is more difficult for the divertor simulation than the limiter simulation because the variation of plosma parameters along field lines is much greater in divertors.

\subsubsection{Physical Regimes of Strong Neutral/Plasma Coupling}

The strongest coupling between the SOL plasma and neutral atoms and molecules occurs in high recycling regimes because the magnitude of the recycling sources in the continuity and energy equations increases in proportion to the plate ion flux. Hence coupling between the b2 and DEGAS codes should be the strongest in these regimes. Divertor tokamaks can achieve larger flux amplification factors than pump-limiter 
tokamaks (up to 1000 in some ITER simulations ${ }^{10}$ ). The use of the b2/DEGAS coupled code set or a similar coupled plasma/neutral calculation may be absolutely necessary to accurately model high recycling divertor experiments. It can be inferred that the use of the coupled codes may not be necessary for the Tore Supra pump-limiter simulation. However the use of the b2 single-species recycling model with the default parameters is inadequate for the pump-limiter simulation because of multiple wall interactions in the pump-limiter throat which are not accounted for in the single-species recycling model. Details of the comparison between the DEGAS calculations and the b2 recycling model calculations will be presented in the next chapter.

\subsection{Two-Species Molecular Physics Recycling Model for the b2 Code}

The two-species molecular physics model was implemented because of the necessity that the free parameters in the single-species model be scaled over plasma input power and transport coefficients. The model description is given here, even though the implementation of the model came from direct conclusions about the single-species model. Presently, this model has teen employed only in the DIII-D calculations. Changes and additions made to this model by the author of this thesis will be emphasized, and described as new options.

This model tracks two species of neutrals: hydrogenic molecules and primary reflected hydrogen atoms. First, reflection coefficients as a function of the incident ion energy are computed for each cell along the plate. The initial calculation ${ }^{31}$ (Hogan) used Eckstein-Verbeeck data, but a new option allows the use of reflection coefficients actually used in the DEGAS code, which come from Janev 33 .

Molecules are tracked first. The neutral molecular flux coming off of the plate in the $n$th cell in the $x$-direction (poloida) is given by:

$$
\Gamma_{x}^{n}=R_{p} * \Gamma_{i}^{n} *\left(1-R_{m}\right) / 2
$$

where

$$
\Gamma_{1}^{n}=\text { incident ion flux at cell } \mathbf{n}
$$




$$
R_{m}=\text { reflected energetic neutral fraction }
$$

This molecular flux is transported upstream (poloidally away from the plate) as described in the single-species recycling model, except that the thermal velocity is computed using a molecular energy of $0.05 \mathrm{eV}$ instead of $15 \mathrm{eV}$, and $\langle\sigma v\rangle_{\boldsymbol{v}}$ is replaced by $\langle\sigma \nu\rangle_{\text {Diss }}$. This model considers the following dissociation reactions in computing $\langle\sigma v\rangle_{\text {DIss }}$ :

$$
\begin{aligned}
& \mathrm{e}+\mathrm{H}_{2}^{0} \rightarrow \mathrm{H}_{2}^{+}+2 \mathrm{e} \rightarrow 2 \mathrm{e}+\mathrm{H}^{+}+\mathrm{H}^{0} \\
& \mathrm{e}+\mathrm{H}_{2}^{0} \rightarrow \mathrm{H}_{2}^{+}+\mathrm{e} \rightarrow \mathrm{e}+2 \mathrm{H}^{0} \\
& \mathrm{e}+\mathrm{H}_{2}^{0} \rightarrow \mathrm{H}_{2}^{+}+2 \mathrm{e} \\
& \mathrm{e}+\mathrm{H}_{2}^{+} \rightarrow \mathrm{H}_{2}^{+}+\mathrm{e} \rightarrow 2 \mathrm{e}+\mathrm{H}^{+}+\mathrm{H}^{0} \\
& \mathrm{e}+\mathrm{H}_{2}^{+} \rightarrow \mathrm{H}^{+}+\mathrm{H}^{0}
\end{aligned}
$$

The number of dissociations occurring in the first cell is computed similar to the way the poloidal deposition is computed in the single-species model:

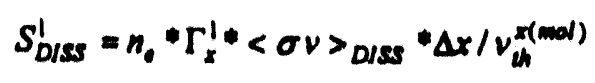

At this point the fraction of dissociation products appearing as ions is computed, and the dissociation ion particle source is stored for each cell. The remainder of the dissociation products in the first cell are Franck-Condon neutrals with a few eV energy, The dissociation source rate is subtracted from the available molecular flux to determine the flux which travels to the next cell. This procedure is continued until all of the available molecular flux has been dissociated in a particular flux tube. Most of the dissociations occur over the first few cells near the plate for the DIII-D simulation.

A new feature recently added is that the Franck-Condons neutrals born from dissociation are transported poloidally after birth to their final poloidal (but not radial) location prior to ionization. This iransport is analogous to the method by which atoms are swept radially in the single-species model and will be described qualitatively here. Starting from the cell on the plate in the same flux tube, a fraction of the Franck-Condons (frup) are transported up the flux tube (analogous to frout). As in the single-species model, deposition in a poloidal cell is based on $\langle\sigma v\rangle$. The poloidal thermal velocity is 
computed based on an input poloidal energy (enex), which ideally is a third of the assumed average Franck-Condon energy. After the neutrals are swept up flux tubes as far as the midplane, the rest of the neutrals are swept down the flux tube, starting from the midplane and going to the plate. If any neutral flux is left after the downstream sweep, neutral flux is swept upstream again until all neutral have been deposited at the poloidal location where they will be ionized. In the original two-species model, the Franck-Condons born from dissociation were not redistributed poloidally.

Next the radial transport of these Franck-Condons is computed. The radial transport is analogous to the single-species radial transport, including the parameter, frout. Neutrals originating at the first wall are modeled in the same manner as the single-species recycling model. The neutral thermal radial velocity is computed based on a radial energy (enerad), which again should be about a third of the Franck-Condon energy. For reasons discussed in the next chapter, a fourth radial (inward) sweep of particles has been added after the third radial (outward) sweep, contingent upon the fact that the there is available remaining neutral flux after the third sweep.

Next the energy source terms are computed for the first species. The electron energy sink is computed as:

$$
S_{\infty}^{n}=S_{i}^{n} * E_{i}^{\text {roms }}
$$

$E_{e}^{\text {bonk }}$ is the average electron energy loss per ionization and was initially computed based on approximations made by the Next European Torus (NET) research team 34 :

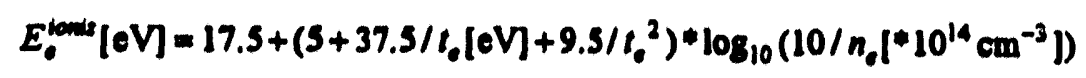

where $T_{e}$ is in $\mathrm{eV}$ and $n_{e}$ is in $10^{20} \mathrm{~m}^{-3}$. This approximation for $E_{c}^{\text {bate }}$ was found to differ with the values in the DEGAS ${ }^{32}$ tables, which are obtained from Janev33. Hence a new approximation has been introduced which better fits the DEGAS data:

$$
E_{c}^{\text {cond }}=17.5+\left(a+b / T_{0}[\mathrm{ev}]+c / T_{a}^{2}\right)^{*} \log _{10}\left(10^{15} / n_{e}\left[\mathrm{~cm}^{-3}\right]\right)^{d}[\mathrm{eV}]
$$


where

$$
\begin{aligned}
& a=-0.9, b=65.9, c=110.4, d=1.72\left|T_{c}<10 e \mathrm{~V}\right| \\
& \left.a=2.79, b=-2.93, c=431, d=2.20 \mid T_{c}>10 \mathrm{eV}\right]
\end{aligned}
$$

In addition, a new electron dissociation power sink has been added to the calculation of electron energy sources. This sink term subtracts 10.5 eV/dissociation for each FranckCondon dissociation that occurs without molecular ionization (eqn. 3.36). For each molecular dissociation that includes molecular ionization (eqn. 3.35, 3.37), the dissociation sink is given by:

$$
\left.S_{\infty}^{\text {DIs }}=15.4+\min \left(1.5 * T_{e}, 10.5\right) \mid \mathrm{eV}\right]
$$

Both of the electron energy dissociation sink terms are approximations based on the dominant processes at a given electron temperature 33 .

Next the ion energy source terms are computed. Originally the calculation was identical to the single-species calculation, i.e. the ion fluid gained 5 eV of energy per ionization, representing the kinetic energy of the Franck-Condon neutrals which were ionized. A better match with DEGAS calculations was obtained using ion energy source terms computed as 4 eV/ion born from dissociation and 7 eV/ion born from electron impact ionization.

A crude charge exchange ion energy source term has also been introduced into the model. The number of charge exchanges that occurs per ionization is computed based on the ratio of reaction rate coefficients $\left(\langle\sigma \nu\rangle_{m} /\langle\sigma \nu\rangle_{w}\right)$. The charge exchange reaction rate coefficient is approximated from the graph in Stangeby35:

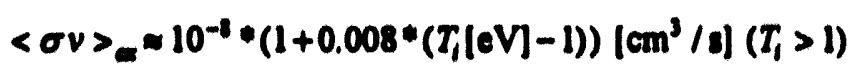

For each ionization occurring in a cell, an ion energy source term from charge exchange is added to the above calculated term:

$$
S_{*}^{\infty}\left[W / \mathrm{cm}^{3}\right]=\frac{\langle\sigma \nu\rangle}{\langle\sigma \nu\rangle_{\omega}} * S_{n}^{m \prime}\left[W / \mathrm{cm}^{3}\right] *\left(\text { foon }-T_{i}[\circ V]\right)
$$

where fcene is the average neutral energy. 
After computing all of the energy source terms, the second neutral species (reflected atoms) is tracked. The tracking of this neutral species is entirely analogous to the single-species model neutral tracking, except that the poloidal energy of the reflected neutral is computed as $3\left(T_{i}+T_{c}\right) R_{w}$, where $R_{n}$ is the reflected energy fraction in a given flux tube, and is obtained from the DEGAS reflection data input table. The fast neutrals are swept upstream poloidally, after which four radial sweeps occur. The radial energy of the neutrals is taken as a third of the Franck-Condon energy to allow fast atomic flux to transport across flux tubes. The electron energy loss per ionization of each of these fast neutrals is given by (eqn. 3.41), and the ion energy gain is simply the energy of the fast neutral prior to ionization. Charge exchanges with fast neutrals are not permitted in the present model.

Convention dictates that the neutral distribution coming off of a plate orthogonal to flux surfaces should be isotropic, which would set both frup and frout at 0.5 . For a tilted plate, frout probably should be scaled in some manner with the cosine of the angle formed by the flux surface normal and the tilted plate. It is assumed that the poloidal energy of the Franck-Condons should be equal to the radial energy, each equal to a third of the average energy (fcene). This average energy is actually in the range of $1-10 \mathrm{eV}$, and the particular value selected $(3 \mathrm{eV})$ fixes the parameters enex, enerad, and fcene. In fact, most of the theoretical predictions of these model "free" parameters were used in the DIIID simulations, and the success of this model in replicating coupled b2/DEGAS results will be discussed in the next chapter.

\subsection{Stairstep Geometry Approximation for Tore Supra Simulation}

The geometric shape of the pump-limiter is difficult to approximate with conventional rectangular grids. In the present study, only the pump-limiter throat is modeled, and it is approximated as a rectangular box. A technique has been devised that approximates each slanted surface of the pump-limiter as a stair-step, i.e. the plasmafacing-surface and the neutralizer plate. For example the atairstep approximation of the 
limiter head is shown in fig. 6. The physical slant of the head is depicted by edge AB, and each "step" represents one cell. The stairstep approximation maintains the orthogonality of the grid so that radial transport is computed properly. This approximation allows the accurate computation of the connection length along the plasma-facing-edge of the limiter head so that the computed neutral ionization mean-free-paths are correct. In addition the parameter frowt in the recycling model can be fixed such that most of the ions striking the head are reflected radially inward. One advaniage of the stairstep method is that the effect of the strong radial gradient of the ion velocity along the limiter head on momentum and energy flow can be examined. These strong radial gradients are caused by the fact that the ions accelerate to the sound speed at different toroidal connection lengths in adjacent fux tubes. Several complications exist with application of the technique, however.

Maintaining the Cartesian orthogonality of the grid requires the use of a large number of grid points in the toroidal direction to resolve the lon flow gradients that occur as the ions accelerate to mach flow at the entrance to the neutralizer plate and limiter head sheaths. This requirement is much more stringent in the stairutep method because only the last few cells need to be concentrated in the toroidal direction near an orthogonal plate. With the stairstep approximation, the concentration of cells must begin a fow cells before the shortest connection length, i.e. the leading edge in the Tore Supra case. Hence the toroidal call width can be only a fow mm above the leading edge, which would more than double the number of mesh points in the toroidal direction from the orthogond grid. Thus the calculation is expected to consume more computer time than present simulations with the orthogonal plates. Moreover the solution technique of the b2 code specified a uniform number of mesh points in both the toroidal and radial directions. Hence regions of the 2-D grid which occur inside the physical pump-limiter (cross-hatched in 6s. 6) must not be allowed to affect the SOL plasm. This "disconnection" of the meah can be accomplished by setting to zero all particle, momentum, and energy flows between the limiter boundary and the limiter body. 
Another issue is the need for a reasonable method of computing the toroidal and radial mean-ffee-paths traveled by neutrals bom at the neutralizer plate and the limiter head prior to ionization. Neo it is unclear how to handle the radial neutral flux impinging upon the "Bat ateps" of the plate (Ag. 6). Hence this technique requires calibration of the came free parameters present in the previously described recycling models internal to b2. and additional parameters a well. The only obvious way to calibrate those parameters is to simulate the entire limiter head and throat geometry with the coupled b2/DEGAS code.

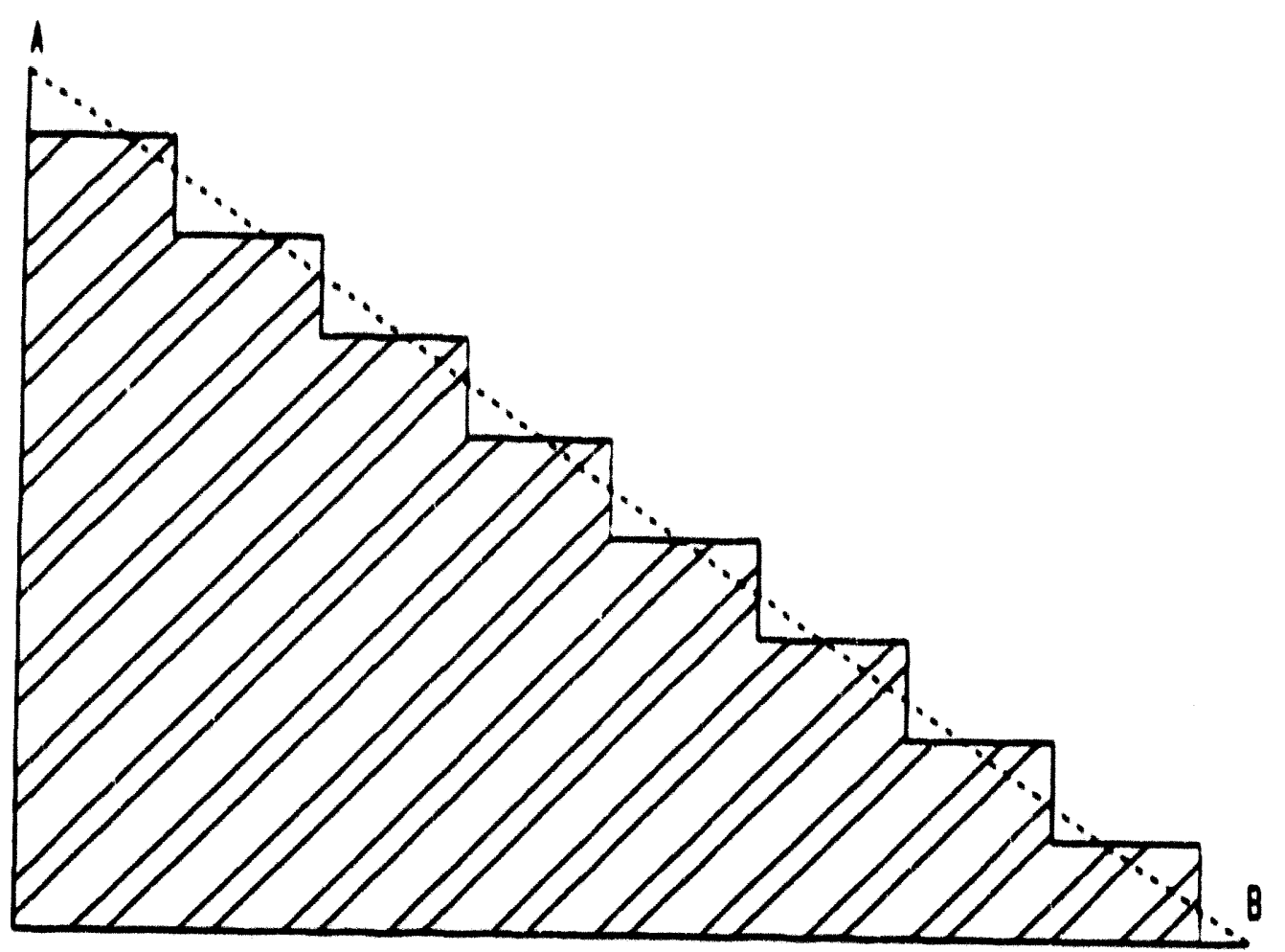

Figure 6: Schematic of aniruep approximation of a pump-limiter head 
In fact an algorithm has been developed to simulate the entire Tore Supra pumpumiter via the atairstep technique. The technique is particularly useful for the Tore Supra tokamak because the slanted edges of both the limiter head and the neutralizer plate can be modeled. The single-species recycling modal has been modified so recycle off plates that are not orthogonal to flux surfaces, with educated guesses for the free parameters. The modal will be teated once an appropriate 2-D grid for the DEGAS code is generated so that coupled b2/DEOAS runs can be done to calibrate the free parameters in the recyeling model. 


\section{CHAPTER 4}

\section{RESULTS AND ANALYSIS}

\subsection{Reaults for the DIII-D tokumak}

As stated in the provious chapter, the b2 code uses anomalous radial transport coeteficients $\left(x_{1}, x_{1}, D_{1}\right)$ which are user-supplied inputs. Other user-supplied inputs into the b2 code which are varied here are the plate recyeling coefficient, $R_{p} ;$ the plauma density, electron temperature, and ion temperature at the inner (core) boundary of the calculation

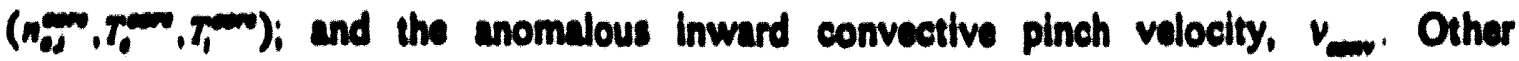
parameter variations which are examined here are the application of a kinetic-baead taceatreaming timit on ion conduction and the use of a non-orthogonal gold in liew of the conventional orthogonal erid. Hence a ceries of b2/DECAS nums was done in which the aforementioned parameteraltechniqueanimits were varied to generate a databace of simulations. The purpose in doing this set of simulations was not to do a comprehensive andyals of each SOL regime created by the variation of input parameters in the b2 code. but to create a databaec of b2/DEOAS caloulation. Each simulation does not necousarily correspond to a particular DII-D ahot. In reality, the present coupled 62/DEOAS model is only as aceurate as the physics in the modals of each code, and this study only attempts to addreas the insocurnetes in the preasent anglo-species recyeling model in b2 and the problems acsociated with doing neutral transpon caloulations whillo maintaining axed plasma conditions in DBCAS. These insecuracies oceur when the codes are run cepanately. Relevent physics which is omitted trom the b2 codo and the censitivity of the DEOAS codo reaults to changes in input atomic and molcoular phyalos cross-ecetions and eurtace reflection coeticients were deacribed briedy in the previous chapter, and the etreet of the limitations of each code on reuults trom this study are diecuened in the next chapter. 
Ater doing the coupled b2/DEOAS runs, the b2 code with the single-species recyeling modal was used to model the ame cases examined by the coupled b2/DECAS model in an efrort 10 fx the adjustable parameters in the single-species model. However it bocame apparent that to match the coupled b2/DEOAS results for different simulations, ecalling of the adjuatable quantities in the aingle-species model with the b2/DEOAS parameter variations would be necessary. It was then discovered that a two-neutralapecies molecular physics recyeling model for use in b2 internally (1.e. without the DEGAS code) was avalablest. The two-species modal was augmented with additional physics and the calculation was refined a deacribed in the previous chapter. Hence the effort to callibrate the tree parameters in the singlo-species model was abandoned and the same tack was initiated with the two-noutral-apecias moleculas physics model. Although the same tree parameter exiat in both of the internal b2 recyeling models, the simulation results from the molecular physics model with conetunt values for frup, frout, feene, enax, and enerad for all of the runs matched the coupled b2DECAS nums suffeciently well so that it was unnecescary to seale these quantitice with variations in 62/DEGAS run parameters.

Then the b2 code with the "callbruted" molocular physice model was uned to analyze two DMJ-D shots, a qulescent S MW input H-mode confinement shot and a 10 MW input $H$-mode confinement shot. The usor-input transport coefficients $\left(X_{1}, \chi_{1}, D_{1}\right)$, the recyeling coeficient $\left(\boldsymbol{R}_{p}\right)$, the convective pinch velocity, and the boundary condition

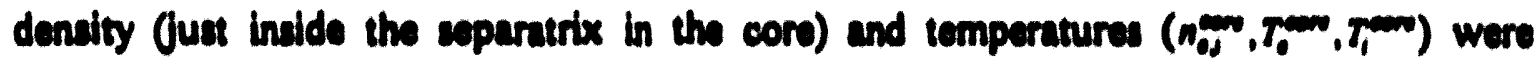
varied to match available divertor door plasma measurements trom DIII.D diagnoatics for the particular shots andyzed. The find reault of the benchmark is the evaluation of the "beat-at" transport and reoyeling coefielents. The benchmark procedure ued here is a trial-and-urror method which entalle doing many runs with input parameter variation to match the meacured divertor plate plaema profilas. The bea match to meacured profles is determined vieually and not by mathematioal moans. The "beat-at" transpont coeficients can be used in aimulations of power-producing devicas which are anticipated to be in the 
same confinement and/or recycling regime, or for simulations of future experiments on present day devices. The b2/DECAS model was not benchmarked againat data from apecibo DIII-D shots because the trial-and-error bench marking procedure used here would require very large amounts of CPU time. The results during each phase of the twospecies model calibration and benchmark procedure for each simulation are discussed here.

\subsubsection{Coupled b2/DEOAS Simulations}

As mentioned in the previous chapter, the firat iteration of the coupled b2/DEGAS technique was done using the b2 single-species recyeling model. The rest of the coupled code iteratione needed for convergence of the simulation were done with the coupled b2/DEGAS model. Nine coupled b2/DEGAS simulations were done, and the parameter variation is tabulated below:

Tnble 2: b2/DEGas Coupled Simulntione Prnmetric Varintione for DIII-D

\begin{tabular}{|c|c|c|c|c|c|c|}
\hline Sim & $x_{1} \cdot x_{1} \cdot D_{\perp}$ & $R_{\text {, }}$ & 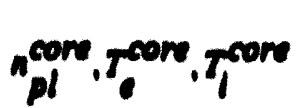 & $v^{2}$ & grid typon & ICFSL \\
\hline$\|$ & {$\left[* 10^{4} \mathrm{~cm}^{2} / \mathrm{s}\right]$} & 11 & {$\left[\mathrm{~cm}^{-3}, \mathrm{eV}, \mathrm{eV}\right]$} & {$[\mathrm{m} / \mathrm{s}]$} & [nom or com] & [yed/no] \\
\hline 1 & $4,0.2,2$ & 0.97 & $2013,100,100$ & -40 & nom & yes \\
\hline 2 & $1,0.05,1$ & 0.97 & $2013,100,100$ & -40 & nom & yes \\
\hline 3 & $4,0.2,2$ & 0.99 & $2 e 13,100,100$ & -40 & nom & yes \\
\hline 4 & $4,0.2,2$ & 0.80 & $2013,100,100$ & -40 & nom & yes \\
\hline $\mathbf{s}$ & $4,0.2,2$ & 0.97 & $3613,300,300$ & -40 & nom & yes \\
\hline 6 & $1,0.05,1$ & 0.97 & $3013,300,300$ & -40 & nom & yes \\
\hline 7 & $4,0.2,2$ & 0.97 & $3013,300,300$ & -10 & nom & yes \\
\hline 8 & $4,0.2,2$ & 0.97 & $3013,300,300$ & -40 & com & 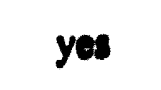 \\
\hline 9 & $4,0.2,2$ & 0.97 & $3013,300,300$ & -40 & nom & no \\
\hline
\end{tabular}

a : orthogonal (nom) or non-orthogonal compreased (com)

b : ion conduction freo-atreaming limit enforced (yea/no) 
The boundary condition plasma density and temperatures used in simulations 1.4 are consistent with measured quantities for $2.5 \mathrm{MW}$ input (NBI) shots. The boundary condition plasma density and temperatures used in simulations 5.9 are consistent with messured quantities for $14 \mathrm{MW}$ input (NBI) shots. The values used for the radial transport coefficients are based on previous benchmarks of the b2 code to experimental data 13,14. Each simulation and the envisioned operating regime are discussed below individually.

\subsubsection{Numerical Convergence of Coupled Code Simulations}

The numerical diagnostics used to determine convergence of the low power base case run (1) are tabulated below as a function of the iteration number, where one run below is defined as one DEGAS calculation followed by a b2 calculation. The convergence pattem of this base case is similar of all of the other coupled b2/DEGAS simulations. Hence this simulation is the only one for which detalled numerical diagnostics during each 62/DECAS iteration are presented. In general the cases with the higheat recycling coefficient and injected power take the longest number of iterations to converge because the neutraliplasm coupling is strongent in those regimes.

Run 0 in the table below corresponds to calculations of the b2 code with its singlespecies recycling model. Convergence was initially reached at run 12, but then it was discovered that different cell volumes had been ueod in the b2 calculations and the DEGAS calculations. Beginning with run 13, the tota DEGAS source term in each cell was used directly in the b2 code, instead of the volumetric source rates (coe the discussion of 2-D meshes and aseociated problems in the previous chapter). Beginning with run 16, the normalization procedure was adjuated to insure that the 62 calculation maintained both the magnitudes of the integrated cource terms and the local proflles of each of the source terms as computed by the DEOAS code, as described in the last chapter. It is estimated that only about half as many b2/DEGAS iterations would have boen nociessary for 
convergence of this run if the procedure to eliminate mesh-oriented problems had been used from the first iteration.

Table 3: Numerical Convergence Diagnostics for Simulation 1(DIII-D)

\begin{tabular}{|c|c|c|c|c|c|c|}
\hline Run & $\left.q_{\text {tas }}^{\text {por }}\right|_{\text {pare }}$ & $\int \Gamma_{\text {lon }}^{\mu t} \int \Gamma_{l}^{p t}$ & & $\left.n_{\text {plas }}^{\text {end }}\right|_{p}$ & $\left.T_{e}^{e x}\right|_{\text {plese }}$ & $\left.T_{1}^{N \infty}\right|_{p}$ \\
\hline \multirow[t]{2}{*}{ \# } & {$\left[W / \mathrm{cm}^{2}\right]$} & 1] & {[]$^{a}$} & {$\left[10^{13} \mathrm{~cm}^{-3}\right]$} & n & $n$ \\
\hline & (in, out) ${ }^{b}$ & (in, out) & & (in,out) & (in,out) & (in,out) \\
\hline 0 & 35,56 & $4.11,5.14$ & $5.6,33,4.5$ & $9.13,7.89$ & $2.9,7.9$ & $5.1,5.0$ \\
\hline 1 & 17,80 & $75,5.28$ & $8.7,48,4.4$ & $21.5,6.24$ & $1.0,1.5$ & $2.1,2.1$ \\
\hline 2 & 36,74 & $3.88,5.52$ & $11,52,7.6$ & $8.65,7.67$ & $3.5,12$ & $5.2,2.9$ \\
\hline 3 & 37,75 & $3.89,5.54$ & $11,52,8.4$ & $8.40,7.67$ & $3.9,12$ & $5.3,3.1$ \\
\hline 4 & 41,82 & $4.67,5.40$ & $12,45,7.0$ & $10.2,7.39$ & $3.9,14$ & $4.5,1.7$ \\
\hline 5 & 63,83 & $4.15,6.26$ & $16,54,10$ & $7.80,9.77$ & $8.3,9.9$ & $3.8,2.8$ \\
\hline 6 & 47,62 & $4.34,6.50$ & $16,75,11$ & $9.23,11.0$ & $5.1,7.6$ & $4.4,3.4$ \\
\hline 7 & 44,66 & $4.51,6.22$ & $14,68,10$ & $10.9,9.77$ & $4.0,9.0$ & $4.2,3.0$ \\
\hline 8 & 35,62 & $4.52,6.12$ & $13,63,6.9$ & $11.6,9.81$ & & $4.2,3.3$ \\
\hline 9 & 38,65 & $4.52,6.05$ & $12,58,7.8$ & $10.4,9.08$ & $3.4,9.4$ & $4.5,3.2$ \\
\hline 10 & 37,73 & $4.54,5.95$ & $11,55,7.6$ & $10.3,8.08$ & $3.4,12$ & $4.5,2.7$ \\
\hline 11 & 36,73 & $4.52,5.87$ & $12,61,7.9$ & $10.5,8.30$ & $3.3,11$ & $4.5,2.7$ \\
\hline 12 & 35,75 & $4.44,5.94$ & $12,57,7.6$ & $10.4,7.77$ & $3.1,12$ & $4.5,2.5$ \\
\hline $13^{\circ}$ & 44,81 & $4.34,5.57$ & $9.4,45,5.8$ & $9.72,6.54$ & $3.6,15$ & $4.6,2.3$ \\
\hline 14 & $.52,80$ & $4.12,5.63$ & $9.6,45,7.5$ & $7.72,6.66$ & $6.5,14$ & $4.6,2.7$ \\
\hline 15 & 55,81 & $4.18,5.69$ & $9.7,45,8.5$ & $7.55,6.72$ & $7.2,14$ & $4.4,2.9$ \\
\hline $16^{d}$ & 47,87 & $4.55,5.22$ & $8.0,38,5.6$ & $9.83,5.50$ & $4.6,18$ & $4.5,2.3$ \\
\hline 17 & 43,86 & $4.45,5.31$ & $9.4,44,8.0$ & $10.3,5.97$ & $3.7,16$ & $4.4,2.7$ \\
\hline 18 & 43,90 & $4.60,5.23$ & $8.9,42,7.6$ & $11.6,5.86$ & $2.7,17$ & $4.2,2.4$ \\
\hline
\end{tabular}

a : units : $\left[\mathrm{cm}^{-3}, W / \mathrm{cm}^{3}, W / \mathrm{cm}^{3}\right]$

b : (in, out) means the inboard value followed by the outboard value

c : DEGAS total source terms in each cell used in lieu of volumetric terms

d : additional normalization of DEGAS source terms to insure that the computed b2 profile is identical to the DEGAS profile 
The final run indicates a $17 \%$ increase in peak heat flux as compared to run 12 . Athough a 17\% difference does not seem large, it is nonetheless larger than the goal of accurately predicting heat flux with an allowed tolerance on the order of the Monte Carlo statistical error $(5 \%-10 \%)$ for the coupled code model. The total Cray-II CPU time used in this single simulation is estimated to be 130 minutes.

The integrated particle flux and peak heat flux on each plate converge, as do the plate plasma density, ion and electron temperatures at the separatrix, and the peak volumetric recycling source magnitudes. The inboard plate plasma density and temperatures on the outer-strike-point (separatrix) exhibit larger variations in the final runs than the other quantities, but these variations are unimportant because the peak inboard heat flux and integrated particle flux are unaffected, and also because the electron temperature is sufficiently low that sputtering of the targets should not be a major problem.

\subsubsection{Results for the Nine Coupled b2/DEGAS Simulations}

Each of the nine coupled code simulations is discussed separately in this section. No expectations of the b2/DEGAS high input power and low input power base case simulations are given. The only part of the discussions dealing with expected results from the non-base case b2/DEGAS simulations is simplistic predictions based on the results from the base case and the variation of parameters from the base case to the particular simulation. The discussions include the reasoning behind selection of the particular parameter combinations used in the simulation and the possible tokamak SOL regimes envisioned through the use of those parameters.

As mentioned earlier, scaling of the adjustable parameters in the molecular physics model was not necessary because usage of the theoretical predictions of these parameters provided a sufficiently good comparison between the b2 code with the molecular physics model and the coupled b2/DEGAS model with the orthogonal grid. To be specific, the following constant values were chosen for the parameters frup, frout, fcene, enex, and 
enerad (see previous chapter for description of parameters): $0.5,0.5,3.0,1.0,1.0$. For the non-orthogonal grid, however, neutral transport is different from the orthogonal grid becesuse neutrals come off the plate with a cosine distribution to the plate normal, not the flux surface normal. The b2/DEGAS calculations indicated that the recycling sources are shifted off the separatrix and are more spread out radially. To compensate for this effect, the following values were used for frup, frout, fcene, enex, and enerad for use with the non-orthogonal grid: $0.25,0.5,3.0,2.0,2.0$. A detailed table of comparisons of separatrix plasma density, temperature, peak heat flux, recycling source terms, and the scale lengths of these quantities between the molecular physics model and the coupled b2/DEGAS run for each simulation is included in the appendices. Included in those tables is a calculation by the molecular physics model in which the electron energy sinks were uniformly increased by $10 \%$ (enhanced radiation) on the inboard side cells to better natch inboard divertor plate peak heat flux from the b2/DEGAS calculation. In general the two-species model appears to underestimate the recycling flux on the inboard side, which of course leads to a calculation of a higher inboard side heat flux than the b2/DEGAS model. This explains why the enhancement of electron energy sinks on the inboard side mentioned above was utilized.

A comparison between the single-species b2 recycling model, the b2 molecular physics recycling model, and the coupled b2/DEGAS model of peak divertor plate heat fluxes and plasma parameters at the separatrix on the divertor plates is also presented in the discussion of each simulation. Particular emphasis is placed on discussions of differences of plasma parameters on the outboard plate since the peak heat flux is usually higher on the outboard plate than the inboard plate. Differences in calculations of separatrix density and temperature are discussed in less detail because the crnvergence of these quantities is not as good as the peak plate heat flux in the b2/DEGAS simulations, typically varying by up to $10 \%-15 \%$ in successive iterations. 


\subsubsection{Simulation 1: low power base case}

The radial transport coefficients for this base case come from the "best-fit" values obtained from a previous b2 code benchmark (without DEGAS) to IRTV heat flux measurements and Langmuir probe plasma density and electron temperature measurements along the outboard divertor plate for a set of $5 \mathrm{MW} \mathrm{NBI}$ power, L-mode and $\mathrm{H}$-mode confinement shots ${ }^{13}$. That benchmark used a single-neutral-species recycling model similar to the one described in the previous chapter. The recycling coefficient comes from a particle balance for similar shots ${ }^{36}$, and the default convective pinch comes also comes from the benchmark in which a strong (anomalous) inward pinch was needed to match the measured steep outboard divertor plasma density profile. The fits of b2 computed plasma parameters to measured values obtained from the early benchmark will be shown in the section of this chapter that deals with benchmarking of the molecular physics recycling model to DIII-D data.

In actuality the plate recycling coefficient approaches unity very quickly during a shot because of the high ion flux in divertor discharges. This plate ion flux quickly fills all of the empty lattice sites in the first few monolayers of the target plate; after saturating the available lattice sites, all ions incident on the plate are sent back into the plasma as backscattered neutrals or cold molecules, which implies an actual plate recycling coefficient of 1.0. In this and other simulations, the plate recycling coefficient is less than unity to allow for wall pumping and anomalous particle loss mechanisms.

Comparisons of divertor plate plasma parameters and integrated particle and energy fluxes between the three different recycling model used is given below. It is significant that the outboard peak heat flux predicted by the single-species model is $40 \%$ lower than the coupled b2/DEGAS model. The reason for this discrepancy is that the recycling particle source and electron energy sink is very large on the separatrix in the cells very close to the plate for the single-species model. Localization of the electron energy sink on the separatrix drops the electron temperature, and the separatrix (peak) 
heat flux is also lowered. In comparison, the two-species model calculation is in better agreement with the b2/DEGAS calculation because a significant fraction of the ions hitting the plate $(\sim 38 \%)$ at the separatrix are reflected as energetic neutrals, which have a long ionization mean-free-path. These energetic neutrals are reionized far away from the plate in regions of higher electron temperature, which reduces the average energy loss per ionization (eqn. 3.41) and the total energy loss due to excitation and ionization. Moreover, both the coupled b2/DEGAS and b2 molecular physics model predict a broader radial ionization profile than the $b 2$ single-species model. This broad profile spreads out the

Table 4: Comparison of Divertor Plate Plasma Parameters (Simulation \#1)

\begin{tabular}{|c|c|c|c|}
\hline parameter & b2/DEGAS & b2 two-species & b2 single-species \\
\hline$q_{\text {cot }}^{\text {et (in, out) }}\left[\mathrm{W} / \mathrm{cm}^{2}\right]$ & 43,90 & 50,82 & 35,56 \\
\hline$n_{e}^{n e p}($ in, out $)\left[\mathrm{cm}^{-3}\right]$ & $1.2 \mathrm{e} 14,5.9 \mathrm{e} 13$ & $8.7 e 13,6.2 e 13$ & $9.6 \mathrm{e} 13,7.9 \mathrm{e} 13$ \\
\hline$T_{a}^{n \prime \prime}$ (in, out) [ev] & $2.7,17$ & $5.7,15$ & $2.9,7.9$ \\
\hline$T_{1}^{\infty p}$ (in, out) [ev] & $4.2,2.4$ & $4.3,2.7$ & $5.1,5.0$ \\
\hline $\int q_{n o t}$ (in, out) [W] & $1.9 \mathrm{e} 5,6.6 \mathrm{e} 5$ & $2.4 \mathrm{e} 5,6.1 \mathrm{e} 5$ & $2.3 \mathrm{es}, 6.0 \mathrm{es}$ \\
\hline $\int \Gamma_{1}$ (in, out) $\left[\mathrm{s}^{-1}\right]$ & $4.6 \mathrm{e} 22,5.2 \mathrm{e} 22$ & $4.0 e 22,5.2 e 22$ & $4.1 e 22,5.1 e 22$ \\
\hline
\end{tabular}

where

$q_{\text {bot }}^{\text {pt }}$ (in, out) - peak heat flux along each plates

$n_{e}^{\text {esp }}$ (in, out) - separatrix density at the inboard and outboard plates

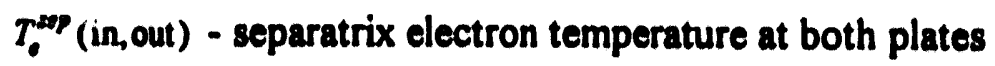

$T_{i}^{\infty N p}$ (in, out) - separatrix ion temperature at both plates

$\int q_{n o t}$ (in, out) - total plasma energy taken into the sheath along each plate

$\int \Gamma_{i}$ (in, out) - integrated particle flux to each plate 
electron energy sink radially and reduces the impact of the energy sink on electron temperature along the separatrix. The afor: 7entioned differences in the radial and poloidal electron energy sink profiles between the recycling models also occur in most of the other simulations, which results in a lower computed peak heat flux by the single-species model in those cases as well.

\subsubsection{Simulation 2: low power and low transport coefficients}

The transport coefficients selected in this case $\left(x_{1}, x_{1}^{\prime}, D_{1}=1.0,0.05,1.0\left[10^{4}\right.\right.$ $\mathrm{cm}^{2 / 8}$ ) imply an improved core particle and energy confinement regime over the low power base case. These coefficients may be applicable to high quality H-mode confinement shots on DIII-D, or maybe even the recently discovered very-high confinement regime ( $\mathrm{VH}$ mode) ${ }^{37}$.

The power $\left(P_{s o L}\right)$ and particles $\left(S_{s o L}\right)$ crossing the separatrix into the SOL from the core is given by:

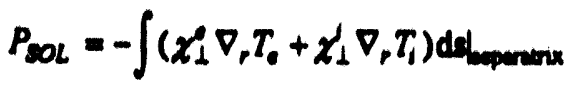

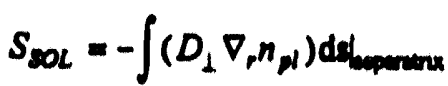

where the radial gradients are taken at the separatrix. Reduction of the radial transport coefficients implies less power and particles and power flowing in the SOL. Hence the plate particle and heat fluxes should be drastically reduced as compared to the low power base case results. The model comparison table below shows that to be the case.

The peak outboard plate heat flux computed by the single-species model is again lower than the coupled b2/DEGAS calculation by $40 \%$ for reasons described in the discussion of the base case. The other relevant difference between the models in this regime is the very low inboard peak heat flux computed by the b2/DEGAS model. This deviation can be attributed to the different approximations of the electron impact ionization reaction rate coefficient used in the two codes. At temperatures below $10 \mathrm{eV}$, the accuracy of the plate reflection coefficients and atomic physics reaction rate coefficients used in the DEGAS calculation and the b2 molecular physics model become 
very suspect due to the lack of experimental data in that low energy range. The electron impact ionization rate coefficient approximations used in all three models are also suspect below $10 \mathrm{eV}$,

\section{Table 5: Comparison of Divertor Plate Plasma Parameters (Simulation \#2)}

\begin{tabular}{|c|c|c|c|}
\hline parameter & b2/DEGAS & b2 two-species & b2 single-species \\
\hline$q_{b t}^{\text {et }}$ (in, out) $\left[\mathrm{W} / \mathrm{cm}^{2}\right]$ & 12,34 & 17,36 & 19,20 \\
\hline$n_{0}^{\infty+1}($ in, out $)\left[\mathrm{cm}^{-3}\right]$ & $5.2 \mathrm{e} 13,3.2 \mathrm{e} 13$ & $4.8 e 13,4.7 e 13$ & $4.5 e 13,5.5 e 13$ \\
\hline$T_{0}^{\alpha s p}($ in out $)[\mathrm{ev}]$ & $1.8,12$ & $3.0,7.4$ & $3.2,3.3$ \\
\hline$T_{1}^{\infty N p}($ in, out) [ev] & $4.5,3.8$ & $5.2,4.8$ & $5.8,6.0$ \\
\hline $\int q_{m i t}$ (in, out) $[\mathrm{W}]$ & $1.1 \mathrm{es,} 2.5 \mathrm{es}$ & $8.904,2.3 \mathrm{es}$ & $9.8 \mathrm{e}, 2.1 \mathrm{es}$ \\
\hline $\int \Gamma_{1}($ in, out $)\left[s^{-1}\right]$ & $1.7 e 22,1.9 e 22$ & $1.5 e 22,2.1 e 22$ & $1.5 e 22,2.4 e 22$ \\
\hline
\end{tabular}

\subsubsection{Simulation 3: low power and high recycling}

In this case the recycling coefficient is increased to approach unity $\left(R_{p}=0.99\right)$ to simulate a true high recycling regime. This case is similar to shots on DIII-D in which anomalous particle losses do not occur. Hindsight indicates that a choice of unity is better to simulate the high recycling regime, but it was feared that use of $R_{p}=1.0$ would lead to a "recycling runaway" and plasma thermal collapse from the strong energy sinks associated with very localized recycling in the b2/DEGAS model. Such an event could occur because of the lack of an immediate feedback system which would immediately reduce the computed recycling source due to the drop of the electron impact ionization reaction rate coefficient because of the drop in electron temperature. This foedback mechanism is usually present only in internal calculations and not in calculations using externally coupled codes (see the previous chapters' description of the disadvantages of the b2/DEGAS model). In any case a favorable comparison between the recycling models in this regime 
would indicate that the b2/DEGAS calculation need not be repeated with unity recycling because of the high probability of a good match with unity recycling.

Comparing this run to the low power base case, a lower peak heat flux and higher plate particle flux is expected. As described earlier, an increase in recycling causes a decrease in electron temperature and plate heat flux, but an increase in plate ion flux. The comparison between models is given below:

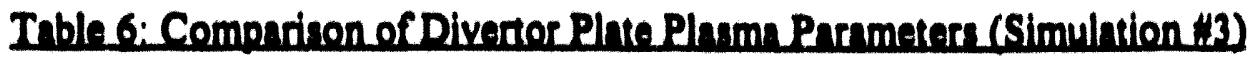

\begin{tabular}{|c|c|c|c|}
\hline parameter & b2/DEGAS & b2 two-species & b2 single-specios \\
\hline$q_{m}^{m}$ (in, out) $\left[W / \mathrm{cm}^{2}\right]$ & 26,82 & 36,72 & 19,49 \\
\hline$n_{0}^{n+1}($ in, out $)\left[\mathrm{cm}^{-3}\right]$ & $1.3 e 14,7.3 e 13$ & $1.0014,7.6013$ & $1.9014,9.5013$ \\
\hline$T_{0}^{\infty N}$ (in, out) [ov] & $1.9,13$ & $3.3,12$ & $1.2,4.6$ \\
\hline$T_{1}^{\infty N}($ lin, out $)$ [ev] & $3.8,2.7$ & $4.6,3.4$ & $2.4,5.6$ \\
\hline $\int q_{m}$ (in, out) [w] & $1.8 \mathrm{es}, 6.6 \mathrm{es}$ & 1.9e5, 6.0es & $1.205,5.4 e 5$ \\
\hline $\int \Gamma_{1}$ (in, out) $\left[x^{-1}\right]$ & $4.8 e 22,5.6 e 22$ & $4.3022,5.6022$ & $5.9022,5.5022$ \\
\hline
\end{tabular}

As in the other cases and for the sume reasons, the single-species model grossly undereatimates the peak heat flux on the outboard side. The two-species model underestimates the heat flux by about $12 \%$, but this difierence may be related to Monte Carlo statiatical error in the DEGAS, portion of the b2/DEGAS coupled code run. This particular run took a large number of iterations to converge and the convergence properties of this run barely meet the convergence criteria.

\subsubsection{Simulation 4: low power base case with low recycling}

In this case the recycling coefficient is set 0.8 to simulate atrong pumping and/or anomalous particle loss. This value of the recycling coefficient was also used in the simulation of $L$-mode shots in the aforementioned benchmark 13 of $b 2$ code calculations to DIII-D data. Compared to the base case, the peak divertor heat flux and separatrix 
electron temperature on each plate are expected to be higher, and the separatrix plauma density at the plate is expected to be lower. Separatrix ion temperature is expected to be a little lower than the base case because the recycling energy source to the ions from the kinetic energy of the Franck-Condons is reduced because of the reduced recycling flux. Comparisons between the various models are shown below:

Table 7: Comparison of Diven or Plnte Plum Prameters (Simulation 4t4)

\begin{tabular}{|c|c|c|c|}
\hline parameter & b2/DEGAS & b2 two-species & b2 single-species \\
\hline$q_{m i n}^{m}($ in, out $)\left[W / \mathrm{cm}^{2}\right]$ & 110,109 & 100,105 & 92,94 \\
\hline$n_{0}^{m+1}($ in, out $)\left[\mathrm{cm}^{-3}\right]$ & $1.9 \mathrm{e} 13,1.7 \mathrm{e} 13$ & $2.1013,1.8013$ & $3.0013,3.1013$ \\
\hline$T_{0}^{\infty}($ In, oul $)$ [ov] & 36,47 & 33,44 & 25,29 \\
\hline$T_{1}^{\infty}($ in, oult) [ov] & $2.9,4.2$ & $2.3,2.0$ & $1.0,1.0$ \\
\hline $\int q_{m}($ in, out $)[W]$ & $4.7 e 5,8.7 e s$ & $4.405,8.405$ & $4.7 e 5,8.305$ \\
\hline $\int \Gamma_{1}$ (in, out) $\left[0^{-1}\right]$ & $2.2022,3.2622$ & $2.3 e 22,3.2 e 22$ & $2.3 e 22,3.3 \in 22$ \\
\hline
\end{tabular}

As expected all three models show good agreement for this low recycling case because the coupling between the plasma and the neutrals is the weakest in this regime and calculations ftom both of the b2 internal recycling models agree with b2/DEGAS simulation results. Aso as expected the predieted ion temperature is very low because of the weak recycling source, as compared to the base case.

\subsubsection{Simulation 5: high power base case}

This simulation used all of the same inputs as the low power base case except that the following values were used as the boundary condition for plasma density and

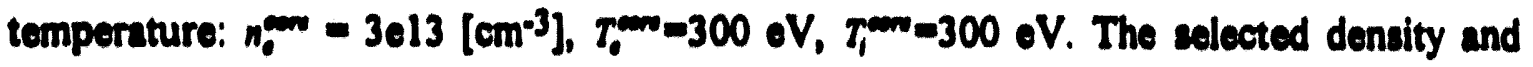
temperature near the separatrix in the core region is not uncommon for shots in which $P_{\mathrm{Nas}}$ is around $14 \mathrm{MW}$. The confinement regime is usually $\mathrm{H}$-mode with a trequent type of 
disnuption known as edge-localized-modes (ELM) for shots in this input power range. The comparison between the various models is given below:

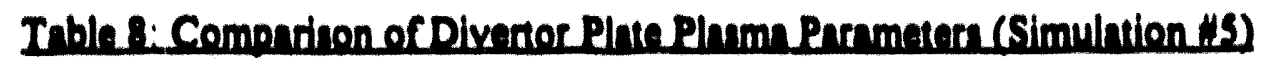

\begin{tabular}{|c|c|c|c|}
\hline parameter & B2/DEGAS & b2 two-species & b2 single-species \\
\hline$q_{t=1}^{\infty}($ in, out $)\left[W / \mathrm{cm}^{2}\right]$ & 1060,960 & 1080,1010 & 1170,900 \\
\hline$n_{0}^{\infty 10}(i n$, out $)\left[\mathrm{cm}^{-3}\right]$ & $5.1013,3.5013$ & $5.2613,3.5013$ & $6.5013,3.1013$ \\
\hline$T_{0}^{\infty}($ in, out $)[0 v]$ & 97,137 & 96,130 & 88,138 \\
\hline$T_{1}^{N e n}($ in, out $)$ [ov] & $<1,<1$ & $<1,<1$ & $<1,<1$ \\
\hline $\int q_{m}($ in, out $)[W]$ & $3.206,5.506$ & $3.206,5.406$ & $3.306,5.006$ \\
\hline $\int \Gamma_{1}($ in, out $)\left[s^{-1}\right]$ & $9.0022,1.0023$ & $9.0022,1.0023$ & $4.8022,6.8022$ \\
\hline
\end{tabular}

As shown above the molecular physics calculations match the b2/DEOAS calculations very well, even for the predictions of separatrix density and temperature at the plates. The fact the two models are almost identical must be considered fortuitous as calculations for the other high power simulations between the two models did not match as well as this one (200 simulations 6-9).

To be noted is the ion temperature at the plate in all three of the simulations. The reason for the very low lon temperature is the use of the ion conduction free-streaming limit. This limit typically reduces the classical parallel ion thermal conductivity, which scales as $\tau_{1}^{2 s}$. Therefore the ion thermal conductivity is limited in a lasge portion of the SOL below the classical valuo in all high input power cases. In order to roughly maintain the same heat flux flowing down a flux tube with and without the free-streaming limit, the ion temperature gradient is necessarily larger when the thermal conductivity is limited. By the time the ions flow down to the plate, the ion temperature has fallen to a support value $\left(10^{-3} \mathrm{eV}\right)$ which prevents thermal collapse on the plate itsolf. The use of classical ion 
thermal conductivity, l.e. no free-streaming ion conduction limit, is examined in simulation 49.

\subsubsection{Simulation 6: high power base case and low transport coefficients}

This case uses low transpont coefficients $\left(x_{1}, x_{1}, D_{1}-1,0,0,05,1,0\left[10^{4} \mathrm{~cm}^{2} / 8\right]\right)$ in conjunetion with high power input. As in the previous low transpon coefficient simulation for the low power case, this combination of parameters implies either a very good $\mathrm{H}$-mode or VH-mode confinement regime. Compared with the high power base case, the heat and particle fluxes to the plate should be much lower than the high power base case run.

Trble 9. Compndeon of Diventor Plnte Plnm Prnmmen (Simulntion.46)

\begin{tabular}{|c|c|c|c|}
\hline parameter & B2/DEOAS & b2 two-species & b2 single-species \\
\hline$q^{\infty}(\mathrm{ln}, \mathrm{out})\left[W / \mathrm{cm}^{2}\right]$ & 460,410 & 440,470 & 420,380 \\
\hline$n_{0}^{m p}(1 \mathrm{ln}$, our $)\left[\mathrm{cm} \mathrm{m}^{-3}\right]$ & $2.3013,2.0013$ & $4.3013,3.1013$ & $1.4613,1.4613$ \\
\hline$T_{e}^{\text {evp }}($ in out $)[\mathrm{ov}]$ & 93,110 & 66,90 & 114,122 \\
\hline$T_{1}^{\infty+\infty}(\ln$, out $)[e v]$ & $<1,<1$ ov & $<1,<1$ ov & $3.2,6.3$ \\
\hline $\int g_{m}$ (in, out) $[W]$ & $9.905,1.806$ & $9.405,1.806$ & $9.805,1.706$ \\
\hline $\int \Gamma_{1}($ in, out $)\left[s^{-1}\right]$ & $2.8022,3.7022$ & $3.2022,4.4022$ & $2.7022,3.7022$ \\
\hline
\end{tabular}

As shown above, the heat flux calculations from both of the internal b2 recycling models acrees fairly well with the b2/DEOAS computed values. However the iwo-species model predicts a higher recyeling regime, which corresponds to higher density and lower temperatures, on both plates. The underlying reason for this diccrepanoy is that the approximation of electron impact ionization and radiation enerey sinks in the two-apecies model was chosen to fit the DECAS databsee of input values for densities between 3.2013 and $1.0014\left[\mathrm{~cm}^{-3}\right]$. The approximation overeatimates the average energy loss per ionization by the electron in the electron impact ionization process below the lower limit quoted above. Hence if the denaity gets below that limit, the eloctron energy sink 
increases, and the electron temperature drops sufficiently low enough to reduce the recycling source magnitude. The resulting plasma density would be a bit higher than the value computed by the b2/DEOAS code, which indeed ceems to be the cave in this simulation. The single species model tends to underestimate the recyeling source magnitude which resulte in the lower densities shown above. In any event, all three modele make aimilar predietions of peak diventor plate heat fux.

\subsubsection{Simulation 7: high power base case and low convective pinch term}

This asee is identical $t 0$ the high power base case oxcept that a very low value of the convective pinch velocity is used: $v_{\text {mon }}=-10 \mathrm{~m} / \mathrm{s}$. An attempt was made 10 use $v_{\text {mov }}$ " $0 \mathrm{~m} / \mathrm{s}$, but this simulation gave convergence problems internal to the b2 code. Hence a low value for the pinch velocity was edected.

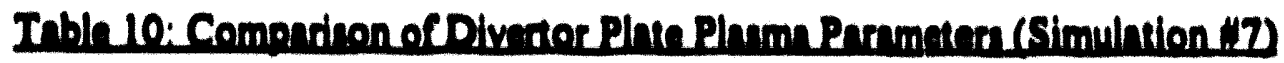

\begin{tabular}{|c|c|c|c|}
\hline paramoter & B2/DEOAS & b2 two-specias & b2 single-species \\
\hline$q^{*}($ in, out $)\left[W / \mathrm{cm}^{2}\right]$ & 1200,1010 & 1210,1140 & 1180,1100 \\
\hline$n_{0}^{m+\infty}($ in out $)\left[\mathrm{cm}^{-3}\right]$ & $7.2013,3.7 \mathrm{el} 3$ & $6.7013,5.0013$ & $1.1014,6.9013$ \\
\hline$T_{0}^{\infty}([n, \infty u t)[(v)$ & 82. 129 & 86,116 & 61,92 \\
\hline${ }_{1}^{\infty}$ (th, our) [ov] & $<1,<1$ & $<1,<1$ & $<1,<1$ \\
\hline $\int q_{W}^{W}(1 n$, out $)[W]$ & $3.3 \oplus 6,6.106$ & $3.506,6.206$ & $3.306,6.066$ \\
\hline $\int r_{1}$ (in out $)\left[v^{-1}\right]$ & $1.9023,1.7023$ & $1.5023,1.8023$ & $1.8023,2.2023$ \\
\hline
\end{tabular}

The efiect of the anomalous inward radial pinch in the b2 code is to make radial divertor protiles of denaty, temperature, and heat aux much more peaked at the coparatrix. The pinch is neaded 13 to obtain a good fit of theas plauma parameters predicted from the b2 code to measured dectron temperature and plaem denaity (ftom Langmuir probes), and divertor heat alux (trom the IRTV) for H-mode confinement shous. The cume profiles during L-mode confinement on DIII.D have been fit 13 with $v_{\text {mov }}=0 \mathrm{~m} / \mathrm{s}$ in a 
aimulation done with an older version of the b2 code. The inward pinch velocity normally offects the outward diffusive velocity 20 as to reduce the plasma radial particle flow velocity to the firat wall. Without the pinch term, the power crossing the separatrix from the core into the SOL increaces because the radial diffusive velocity increases. Hence the increased power into the SOL without the pinch term translates to increased particle and heat flux on the divertor targets.

\subsubsection{Simulation 8: hich power base case and non-orthogonal grid}

The non-orthogonal grid used in this simulation is shown in (fis. 7). The grid was generated based on magnetic flux measurements and subsequential calculations by the EFITD 38 program. The procedure to generate orthogonal grids from EFITD calculations is diecusced eleowheres, and the non-orthogonal grid-generation technique is a modification of that procedure. A description of these procedures is eiven in the appendices.

The modeled outbourd divertor plate runs along the inue vescel foor at a full $45^{\circ}$ tift with respect to poloidal Aux eurfeces. The physical inbourd divertor plate on the DIIID tokamak usually runs along the edge of the inner comer which is almost normal to poloidal fux surfaces. Hence, there is very little difference between the inbourd aide of the non-orthogonal grid and the orthogonal grid. The x-point to plate diatance was selected to be the sume as the correaponding lengths for the orthogonal grid when constructing the non-orthogonal grid.

As mentioned eartier heat flux calculations baead on an orthogonal goid ase simply multiplied by the cosine of the angle the flux wurfhces make with the true plate (fig. I from chapter 1) to obtain the heat fux projection on the true plate. This reduction of heat flux occurs because the aree over which the hat is tranaported in a aux tube on the plate is inereased, thus decreasing the flux. One problem with the simple extrapolation is that the seometry of the sepanarix and the floor plays an important role in determining where reoyeled neutrals are ionized. Incident ions to the divertor plate which are emitted as 
molecules have a cosine distribution with the normal to the target (segment BCE of fig 1 from chapter 1), not the normal to the separatrix (segment CD of fig. 1). Hence the region in which most of the neutrals are re-ionized of dissociated can be shifted radially away from the separatrix. The electron energy sink at the separatrix can be reduced, which prevents the electron temperature at the separatrix from falling as much as it would if the source were peaked on the separatrix. Therefore the total plasma energy carried in the flux tube along the separatrix can actually increase. The plasma energy is distributed over a larger area at the plate as compared to the orthogonal grid so that the energy flux is reduced, but this reduction may be much less than the simple extrapolation of the heat flux would predict.

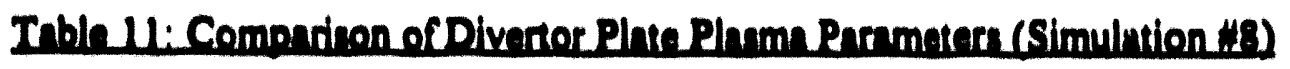

\begin{tabular}{|c|c|c|c|}
\hline parameter & B2/DEOAS & b2 two-species & b2 single-species \\
\hline$q^{n t}($ in, out $)\left[W / \mathrm{cm}^{2}\right]$ & 970,820 & 1060,930 & 960,800 \\
\hline$q_{m}^{\infty}($ in, out $)\left[W / \mathrm{cm}^{2}\right]^{a}$ & 1060,680 & 1080,710 & 1170,640 \\
\hline$n_{c}^{m+1}($ in, out $)\left[\mathrm{cm}^{-3}\right]$ & $3.0013,6.2013$ & $5.2013,4.6013$ & $2.9013,2.6 e 13$ \\
\hline$T_{0}^{+\infty 1}($ lin, out $)$ [ev] & 124,87 & 94,107 & 129,137 \\
\hline$T_{1}^{\infty \prime \prime}($ lin, out $)$ [ov] & $<1,<1$ ov & $<1,<1$ ov & $<1,<1$ \\
\hline $\int q_{m=1}^{N}(L, 0 u t)[W]$ & $3.006,5.606$ & $3.006,5.006$ & $3.006,5.406$ \\
\hline $\int \Gamma_{1}($ in, out $)\left[s^{-1}\right]$ & $6.3022,1.7 \times 23$ & $8.9 e 22,1.1023$ & $6.1022,9.8022$ \\
\hline
\end{tabular}

a : extrapolated from the base case (simulation 5) by multiplying the outboard peak heat Aux by $\cos \left(45^{\circ}\right)$, the approximate incidence angle of poloidal flux tubes with the DIII-D divertor outboard target plate

As shown above the predieted heat flux on the outboard side computed by multiplying the actual heat flux in the high power base case by the cosine of the incident 
angle is much lower the heat flux computed by non-orthogonal grid calculations. Compared to the base case tabulation, the electron temperature indeed increases, ostensibly due to a decrease in the recycling source at the separatrix.

At this point, a discussion of the plasma radial transport inaccuracy caused by the non-orthogonal grid is presented. The limit on the accuracy of the non-orthogonal grid results is the plasma radial transport calculation. Consider a cell that has two opposite edges ( 1 and 2 ) lying along adjacent poloidal flux surfaces. The solution technique of the b2 code assumes that the other two boundaries of the cell (edges 3 and 4) are exactly orthogonal to edges 1 and 2 , and the code computes a plasma radial velocity $\left(v_{\mathrm{ram}}\right)$ in the cell based on that assumption. In the non-orthogonal grids used for the DIII-D simulations, however, edges 3 and 4 are not normal to flux surfaces, and the computed radial velocity has only a component in the actual direction orthogonal to flux surfaces. This component is given by $v_{\mathrm{rad}} \sin (\alpha)$, where $\alpha$ is the average angle between the cell boundaries 3 and 4 and the normal direction to poloidal flux surfaces. The magnitude of the inaccuracy scales with the angle $\alpha$. The component of the computed radial velocity in the parallel direction for a non-orthogonal grid is given by $v_{\mathrm{red}} \cos (\alpha)$, but this effective increase in the parallel transport velocity is small compared to the computed parallel flow velocity. For the DIII-D non-orthogonal grids, the cell boundaries in the poloidal direction are selected to coincide with flux surfaces. The grid-generation procedure allows the inboard plate to be non-orthogonal to poloidal flux surfaces also, provided the flux surfaces intercept the divertor floor instead of the $45^{\circ}$ inner comer plate. It was fortuitous that the inboard flux surfaces intercepted and were approximately normal to the $45^{\circ}$ inner corner plate in all of the non-orthogonal grids used in this study, and the only significant non-orthogonal portion of the grids occurred on the outboard side. It should be noted that the grid-generation procedure computes cell volumes accurately for both orthogonal and non-orthogonal grid cells. The cells just below the x-point on the outboard side are only slightly non-orthogonal (i.e. the anglo $\alpha$ is very small), and the non-orthogonality of the 
cells increases monotonically from the x-point to the plate. Along the plate, the angle $\alpha$ approaches $45^{\circ}$, and the magnitude of the radial flow velocity computation inaccuracy is the largest for these cells. However, the plasma parallel transport dominates the radial transport in the cells near the plate anyway, so the radial transport inaccuracy is expected to have a negligible impact on computed plasma parameters. Unfortunately, the magnitude of this inaccuracy is presently unknown.

One interesting feature of the non-orthogonal grid simulation is the small ratio of inboard to outboard plate particle flux. This can be explained by the fact that the particle and electron energy recycling source peak on the separatrix is higher on the outboard side of the non-orthogonal grid than the orthogonal grid. Consequently the separatrix outboard plate electron temperature is lower in the non-orthogonal grid. Momentum balance along the separatrix initiates a net particle flow from the inboard side to the outboard side to increase the outboard side plasma density to insure approximately constant pressure. This flow decreases the inboard side separatrix density, which decreases plate flux. The in/out plate flux ratio is smaller in the b2/DEGAS calculation than the other models because the electron energy loss per ionization and the total separatrix electron energy sink term is higher, which leads to a lower separatrix electron temperature and a larger flow from the inboard side to the outboard side for pressure balance.

\subsubsection{Simulation 9: high power base case and no ion conduction limit}

The ion conduction free-streaming limit is not enforced here, and all other parameters are identical to the high power base case (simulation 5). The application of this limit is described in earlier chapters and will not be discussed again in here. The removal of the limit should allow ions to carry a large fraction of the total heat down to the plate.

As shown below, the total peak heat flux at the separatrix does increase when the free-streaming limit is removed. The Spitzer-Harm parallel ion heat flux is given by39:

$$
q_{i}^{\prime}=-k_{j}^{\prime} \nabla_{1} T_{i}
$$


where all of the above terms are defined in chapter 3. Because the parallel thermal conductivity of the ions at the separatrix is not limited by the free-streaming-limit and therefore larger, the required temperature gradient to carry the same amount of energy in the ion channel is necessarily smaller. The boundary condition at the inner (core) boundary maintains electron temperature at a high level upstream of the plate, so that the electron temperature at the plate is increased. The plate peak ion heat flux, which scales as $T_{i, s p p}^{1 \text {, }}$, therefore increases also. The total heat flux increases by almost $50 \%$ in the molecular physics model as compared to the b2/DEGAS model, the reason for which is not clear. It is possible that the molecular physics model simulation is not converged, although the required convergence criteria internal to the $b 2$ code were satisfied. However, examination of the solutions at several different time steps indicated changing plasma density and temperature profiles along the plate, although the peak heat flux was relatively unaffected. Confirmation of the convergence of this particular molecular physics (and single-species) model simulation requires further investigation.

Table 12: Comparison of Divertor Plate Plasma Parameters (Simulation \#9)

\begin{tabular}{|c|c|c|c|}
\hline parameter & b2/DEGAS & b2 two-species & b2 single-species \\
\hline$q_{\text {sot }}^{\text {th }}$ (in, out $)\left[W / \mathrm{cm}^{2}\right]$ & 1150,1160 & 1530,940 & 1090,1140 \\
\hline$q_{b o t}^{\alpha k}($ in, out $)\left[W / \mathrm{cm}^{2}\right]^{\mathrm{u}}$ & 1060,960 & 1080,1010 & 1170,900 \\
\hline$n_{e}^{\infty 1 p}($ in, out $)\left[\mathrm{cm}^{-3}\right]$ & $4.8 \mathrm{e} 13,5.5 \mathrm{e} 13$ & $7.0 \mathrm{e} 13,5.6 \mathrm{e} 13$ & $7.2 \mathrm{e} 13,6.1 \mathrm{e} 23$ \\
\hline$T_{a}^{\infty+\infty}$ (in, out) [ev] & 101,105 & 92,87 & 78,61 \\
\hline$T_{i}^{\infty+p}$ (in, out) [ev] & $2.6,4.6$ & $14.0,1.9$ & $<1,7.5$ \\
\hline $\int q_{\text {mot }}^{N}($ in, out $)[\mathrm{W}]$ & $3.2 e 6,5.9 e 6$ & $3.5 e 6,5.6 \mathrm{ec}$ & $3.1 e 6,5.7 e 6$ \\
\hline $\int \Gamma_{i}($ in, out $)\left[s^{-1}\right]$ & $7.7 e 22,1.1 \mathrm{e} 23$ & $8.0 \mathrm{e} 22,1.0 \mathrm{e} 23$ & $9.6 \mathrm{e} 22,1.3 \mathrm{e} 23$ \\
\hline
\end{tabular}




\subsubsection{Effect of Core Boundary Condition}

Unfortunately the boindarv condition used along the core boundary (fixed plasma density and temperatures) allows the power efflux from the core into the SOL to vary when any parameters, including the recycling model, are varied. The magnitude of the core power efflux obviously has a large impact on the SOL plasma conditions because the core represents the energy source for the SOL, and any future simulations should incorporate the core boundary condition which fixes the power efflux (see section on b2 code boundary conditions in chapter 3 ). The core power efflux for all of the simulations and recycling models is tabulated below:

Table 13: Comparison of Core Power Efflux for Simulations 1-9

$\begin{array}{cccc}\text { Simulation \# } & \text { b2/DEGAS [W] } & \text { b2 two-species [W] } & \text { b2 one-species [W] } \\ 1 & 1.95 \mathrm{e} 6 & 1.89 \mathrm{e} 6 & 1,89 \mathrm{e} 5 \\ 2 & 6.82 \mathrm{e} 5 & 6.75 \mathrm{e} 5 & 6.32 \mathrm{e} 5 \\ 3 & 1.86 \mathrm{e} 6 & 1.83 \mathrm{e} 6 & 1.85 \mathrm{e} 6 \\ 4 & 2.25 \mathrm{ee} & 2.19 \mathrm{e} 6 & 2.21 \mathrm{e} 6 \\ 5 & 1.10 \mathrm{e} & 1.10 \mathrm{e} & 1.08 \mathrm{e} 7 \\ 6 & 3.43 \mathrm{e} 6 & 3.43 \mathrm{e} 6 & 3.15 \mathrm{e} 6 \\ 7 & 1.33 \mathrm{e} & 1.38 \mathrm{e} & 1.33 \mathrm{e} 7 \\ 8 & 1.12 \mathrm{e} & 1.13 \mathrm{e} & 1.07 \mathrm{e} \\ 9 & 1.13 \mathrm{e} & 1.14 \mathrm{e} 7 & 1.11 \mathrm{e} 7\end{array}$

Ideally the power efflux for all of the low power simulations (1-4) for all three models should be identical, as should the efflux for all of the high power cases (simulations 5-9). The table above shows that part of the $10 \%$ reduction in outboard plate peak heat flux in the b2/DEGAS simulation 3 (as compared to the base case simulation 1) probably occurs because the rore power efflux decreases by $5 \%$. Similarly the $22 \%$ increase in 
outboard plate peak heat flux in the b2/DEGAS simulation 4 is partially caused by the $15 \%$ increase in the core power efflux. Hence the variation of recycling coefficients should not have as strong of an impact on SOL parameters as indicated in the tables.

The variation of the core efflux is largest when the assumed radial electron and ion thermal diffusivities are varied (simulations 2 and 6 above) because the power flowing into the SOL is dependent directly on these values (eqn. 4.1). An accurate examination of the transport coefficient variation simulations should hold the core power efflux constant. Valid comparisons between the different recycling models can be made for the same simulation, nonetheless, because the core efflux changes by only a few percent in most cases. The largest differences $(\sim 10 \%)$ between the core efflux computed by the three recycling models occurs with the variation of radial thermal diffusivities.

\subsubsection{Benchmark to DIII-D Data}

The previous sections describe the success of the molecular physics two-species recycling model in matching heat flux predictions with the coupled b2/DEGAS calculations. In most of the above simulations, the molecular physics model predictions of the plasma density and temperature conditions existing at the divertor plates also compare well with coupled b2/DEGAS simulations. The b2 code using the molecular physics model internally is now used to simulate two DIII-D shots: one low power input case (5 MW) and one high power case (10 MW). The DEGAS neutral transport calculation is now taken out of the modeling loop. The two shots modeled here are selected specifically because previous benchmarks 13,14 with the $b 2$ code using the single-species recycling model, as well as Langmuir probe and IRTV data, are available for these shots.

\subsubsection{Low Power Input Shot (5 MW)}

\subsection{Brief Shot Description}

Figure 8 shows a few relevant traces during the low power shot, \#66821. The plasma current (IP trace) is held fixed at 1.2 MA and the neutral beam injected power (pinj trace) at $4.5 \mathrm{MW}$ begins at $\mathrm{t}=2200 \mathrm{~ms}$. The onset of $\mathrm{H}$-mode confinement is indicated at 
about $t=2420 \mathrm{~ms}$, at which time the photo diode signal decreases in amplitude and the lineaveraged density (density trace) begins to increa. \&. The data from the divertor floor Langmuir probes corresponding to the outer divertor plate in simulations and the IRTV is chosen at $t=2880 \mathrm{~ms}$, prior to onset of the disruption (giant ELM on phd04 trace) at t=2950ms.

\subsection{Previous Benchmark Results}

The 2-D grid used in the 1990 benchmark was an analytic grid generated by the b2 code, and the magnetic field topology is based on an approximation also in the b2 code. The analytic grid was used because EFITD-based grids were not available at the time. As mentioned previously, the following parameters were varied to fit measured plasma

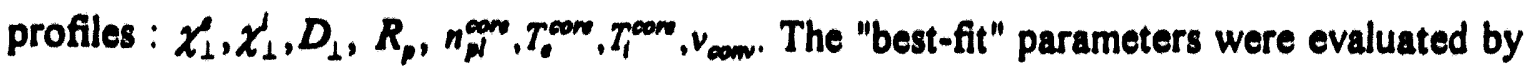
visual inspection of computed and measured profiles and not by statistical methods. The values of the aforementioned parameters that best replicated experimental data are tabulated in the next section. Density, temperature, and heat flux profiles along the outer divertor plate computed by the b2 code using a single-species recycling model similar to the one described in chapter 3 are shown ${ }^{13}$ in fig. 9, along with Langmuir probe and IRTV data. As shown in the above figure, the simulation is in good agreement with the experimentally measured plasma conditions of shot 66821 at $t=2880 \mathrm{~ms}$. The measured electron temperature is significantly than the predicted value far away from the separatrix (which occurs at a displacement of $0 \mathrm{~m}$ on the $\mathrm{x}$-axis) because the boundary condition used in the simulation requires $T_{c}=2 \mathrm{eV}$ at the outer wall (which occurs at the right end of the $x$-axis). The difference in measured and computed plasma density at the outer wall is also a boundary condition effect.

\subsection{New Benchmark Results}

The same shot has been modeled with the b2 code using the new internal twospecies molecular physics recycling model. Two simulations using different 2-D grids have been done. The first simulation used a 2-D grid generated based on magnetic flux 


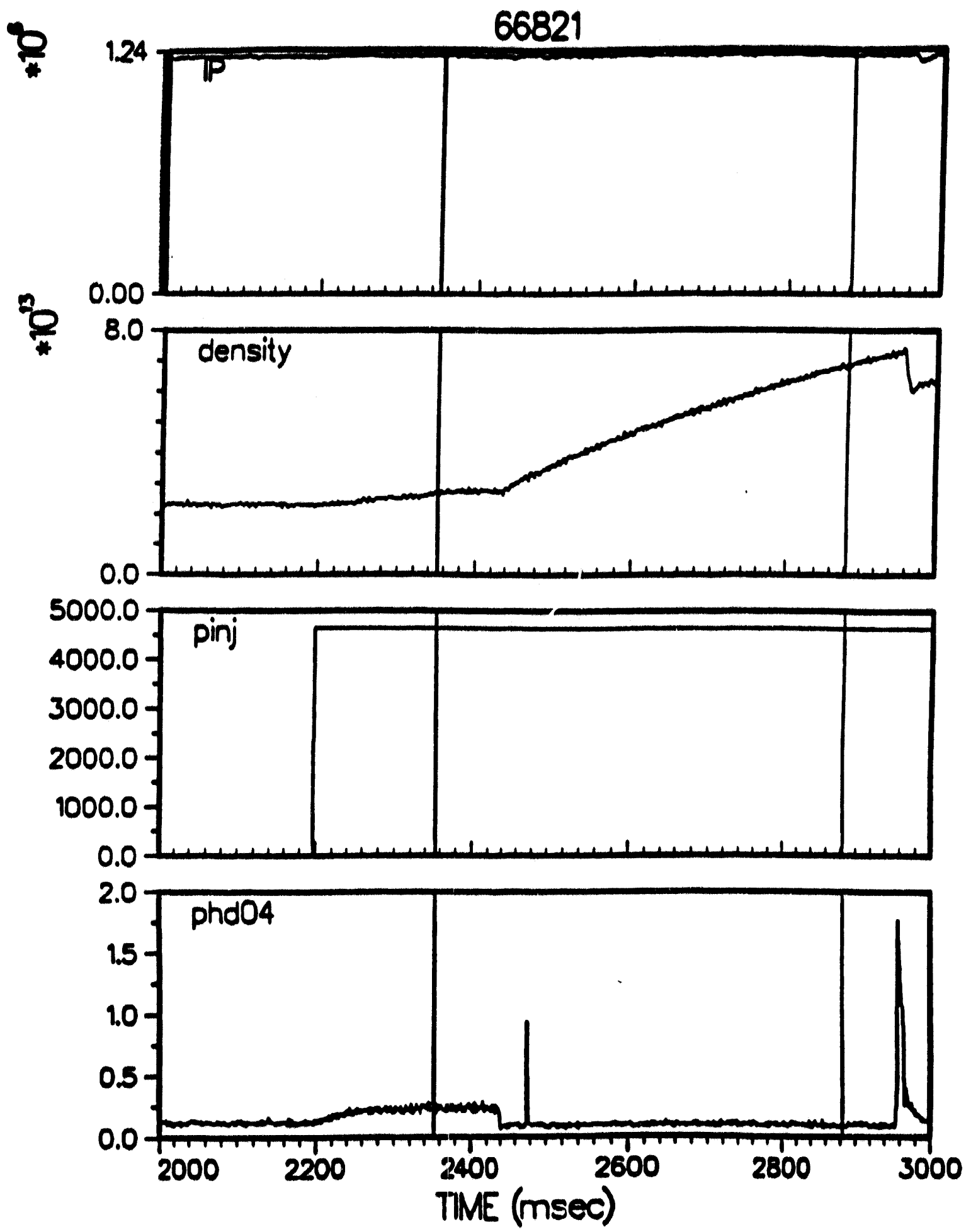

Figure 8: Relevant characteristics 13 of shot 66821 
82 code run 12 If

H-mode Langmuir Prabe Data

shots $66821-32$

IRTV Doto
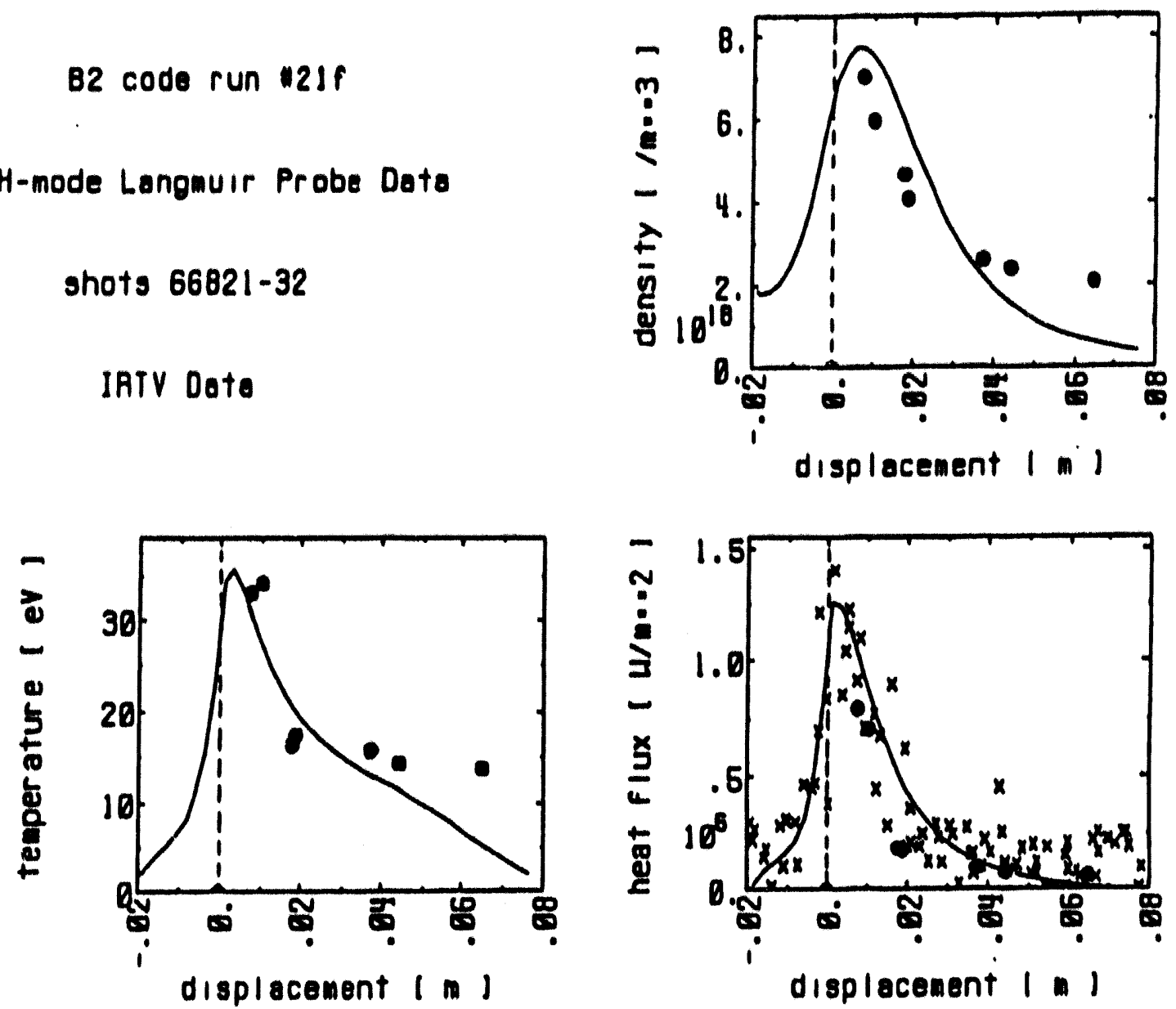

Figure 9: 1990 benchmark'13 profiles and comparisons to data. The solid lines represent code calculations, the dotted points are Langmuir probe measurements, and the x's are IRTV measurements. 


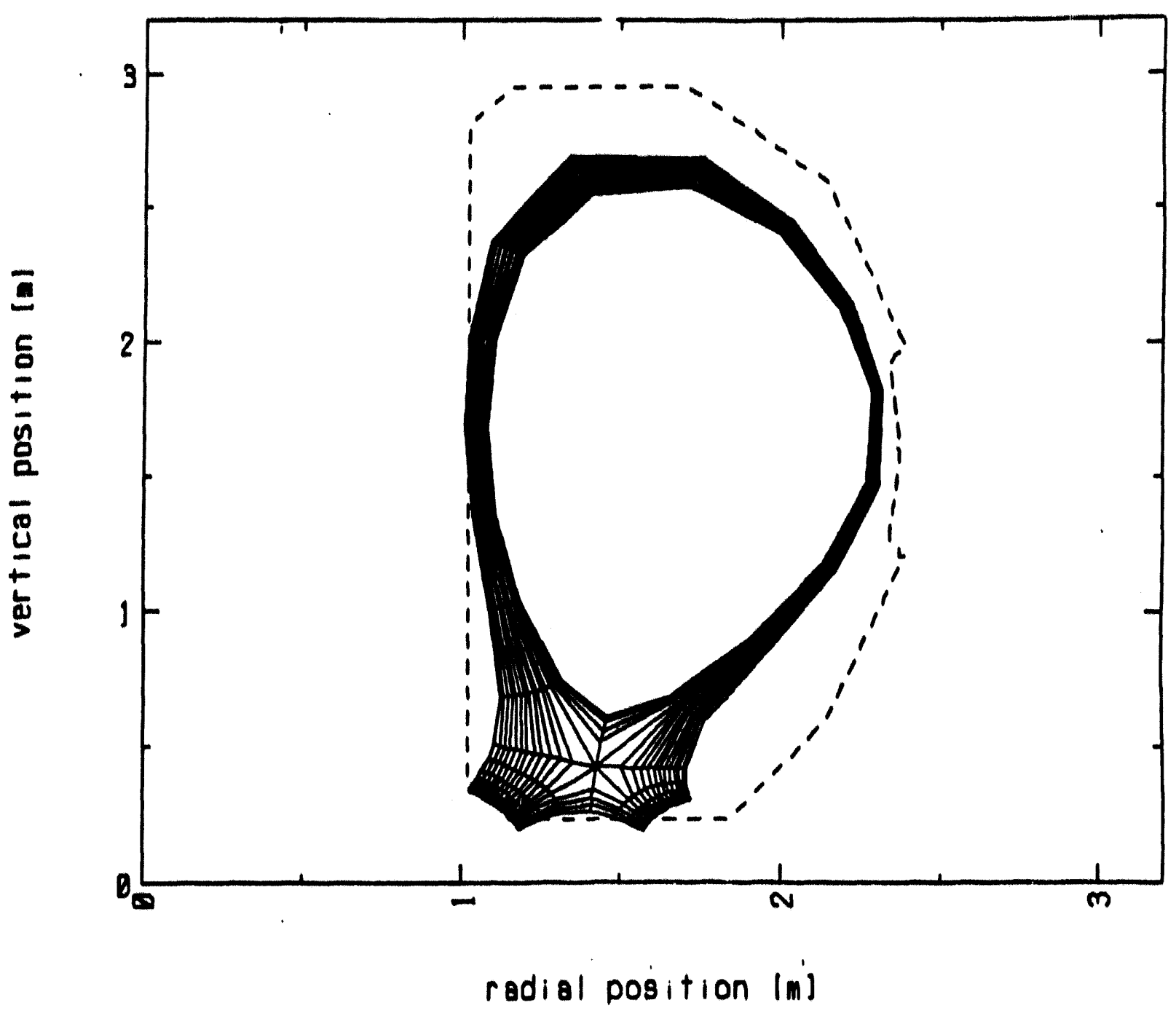

Figure 10: Orthogonal grid used for shot 66821 in this research. The vessel wall is outlined for reference. 


$$
\text { Q }
$$


measurements and fits by the EFITD code for shot 66821 at $t=2880 \mathrm{~ms}$, so that the magnetic field topology corresponded to the shot conditions (fig. 10). The second grid used was a non-orthogonal grid in which the outboard divertor plate ran parallel to the true vessel floor (fig. 11). The same inputs that were varied in the previous benchmark were varied in these simulations. The results of each simulation are discussed below.

The orthogonal grid used in this simulation is EFITD-based and corresponds to the magnetic topology of shot 66821 at $t=2880 \mathrm{~ms}$. The radial distance to the first wall from the outboard strike point is assumed to be about $8 \mathrm{~cm}$, which corresponds to the grid used in the previous benchmark simulations. The evaluation of the "best-fit" input parameters was based on primarily matching the heat flux and electron temperature measurements for reasons described in the section on experimental uncertainties.

The computed density, temperature, and heat flux profiles along the outboard plate are shown below for both the orthogonal and non-orthogonal grids; several experimental data points have been approximated and superimposed for comparison purposes:

-1 Pleomo Dendity ve, Ploto Aedlus

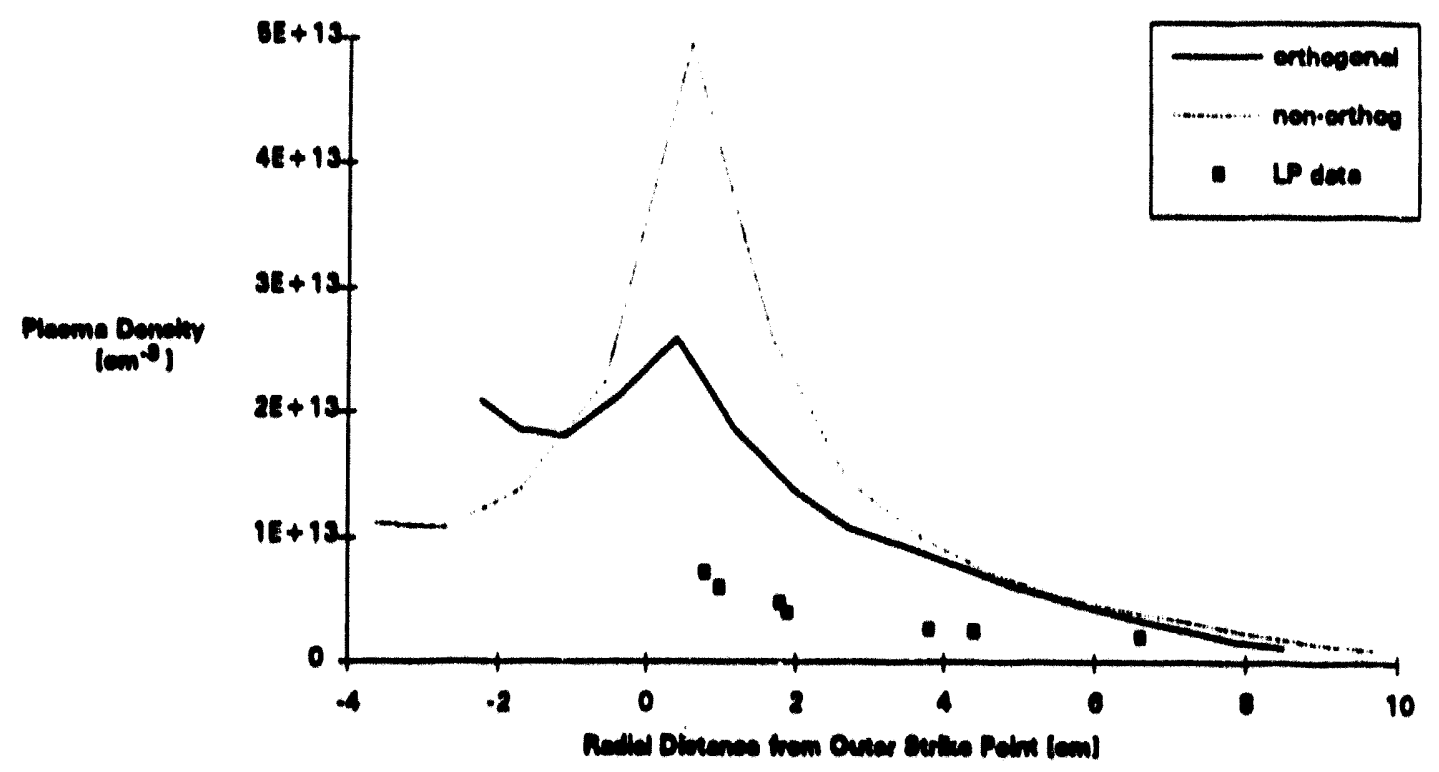




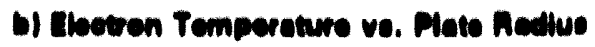

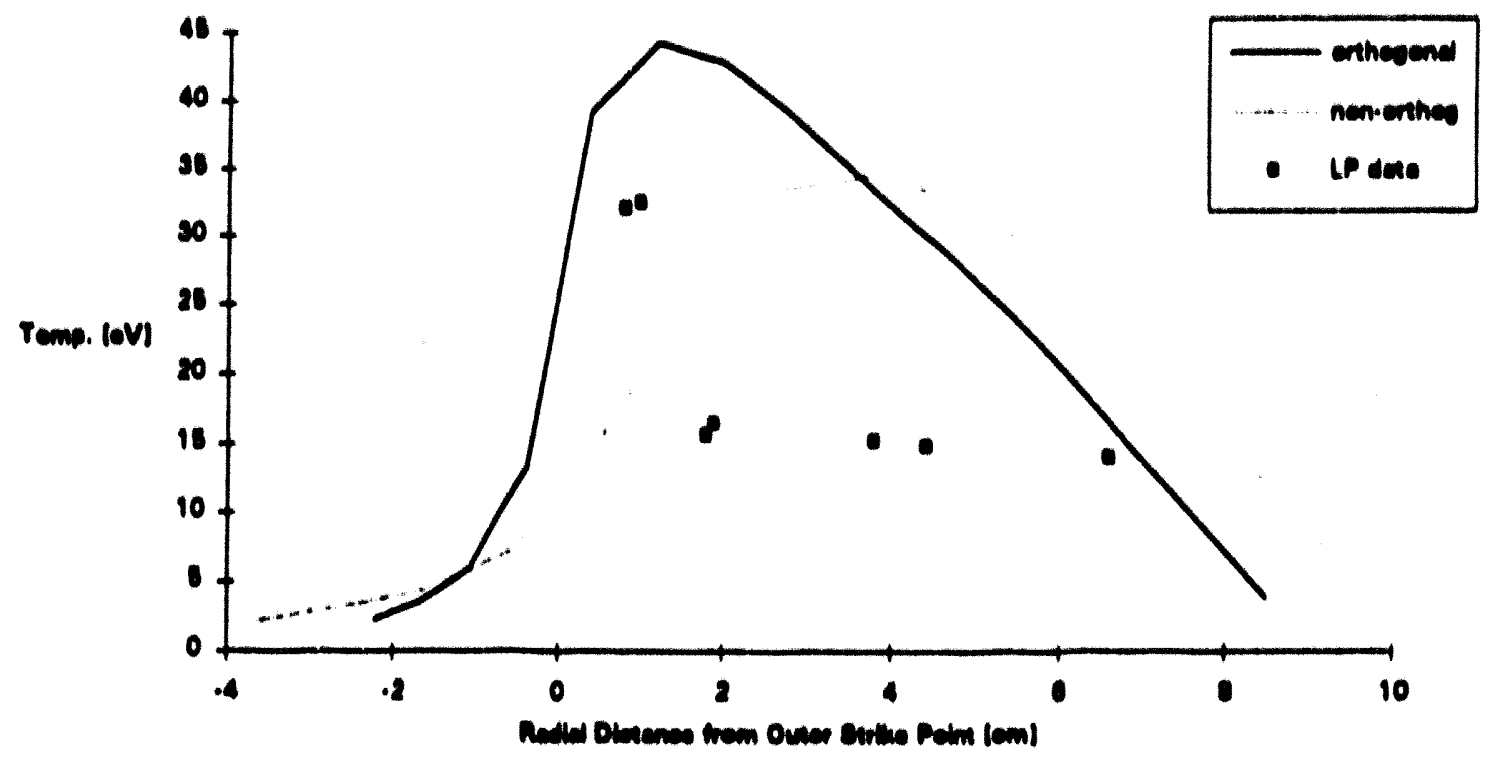

el Plate Hoot Pux vo. Rective

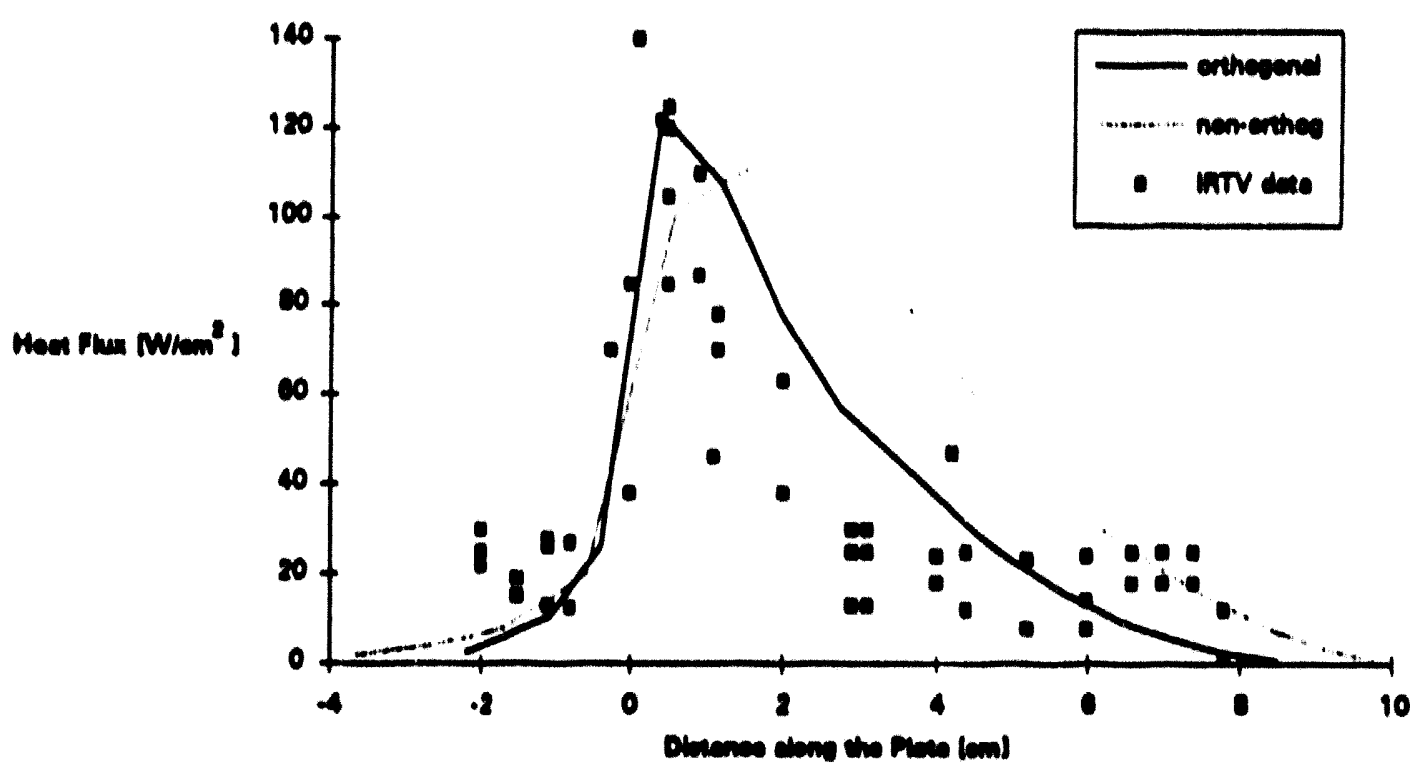

Figure 12: Comparicons of (a)plama denaity, (b)electron temperature, and (c)plasma heat flux profiles along the outboard divertor plate between experimental data from the low power shot and both the orthogonal and non-orthogonal grid simulations 
The most striking difference in the computed profles between the previous benchmark and the present one occurs in the peak density at the plate, which is a factor of three higher than the measured value and the value obtained from the previous benchmark. The magnitude of the density in this simulation is necessary 10 match the IRTV heat flux data. The required peak density was lower in the 1990 benchmark for the following reasons. Firat the field line pitch value $\left(\frac{B_{e}}{B_{m}}\right)$ was $50 \%$ higher at the divertor plate than the value used in the present benchmark. The pitch used in the 1990 benchmark was based on the analytic grid and magnetic field generation intermal to the b2 code; EFITD-based grids were not available at the time. The poloidal heat flux incident on the divertor plate is related to the parallel heat flux and field line pitch by:

$$
9 S^{\prime}=9 P \cdot \frac{B C}{B C}
$$

Therefore the computed heat flux was actually higher than it should have been, and rerunning the simulation with the correct magnetic geometry resulted in the peak heat flux being lower by a factor of 4 from fig. 9 . To match the messured heat flux without affecting the computed electron temperature from the 1990 benchmark (and using the correct magnetic geometry), a higher plasma density than shown in 68. 9 would be needed. Another reason that the peak plate density is lower in the 1990 benchmark is that no free-atreaming limit on ion conduction was used in that simulation, which allowed the ion fluid to carry roughly $40 \%$ of the total plasma energy into the sheath. In the analyais done here, the ion free-streaming limit is enforced, and the ion temperature at the plate is significantly reduced, which leads to a much smaller ion heat dux at the plate. The electron Aluid in this aimulation carries a larger fraction (295\%) of the total plasma thermal energy into the sheath. This increase in the electron heat flux (without affecting the eloctron temperature) requires an increase in the plasma density as compared to the 1990 simulation, which is evident in the present simulation. 
In this simulation, as in the previous benchmark, the plasma denaity and electron temperature fall off more sharply in the outer SOL than indicated by the data because of the boundary conditions imposed at the outer wall boundary. However the measured temperature profile falls off more sharply than the computed profile near the separatrix aleo. A better match to data may be obtained by extending the radial width of the 2-D grid used in the simulation at the plate closer to the true firat wall of the vessal, which would reduce the impact of the boundary conditions in the calculation. It should be mentioned that extending the radial width of the plate all the way to the outer (physical) wall caused numerical convergence problems in the b2 code in this run because of the very low plasma densities and temperatures occurring in that part of the SOL. Even if the numerical convergence problems are solvable, more grid points in the radial direction are needed to insure adequate resolution, which increases the calculation time of the simulation. Another method to reduce the impact of the boundary conditions would be to use alternate boundary conditions which do not impose pedestal temperatures and zero radial particle flux at the firat wall, as described in chapter 3.

The non-orthogonal grid uced in this simulation was also generated from IFITD calculations of the magnetic topology of ahot 66821 at t-2880ms (6g. 11). The heat alux radial profile along the outboard plate computed in this simulation is broader than the orthogonal grid simulation because the electron temperature profile is very broad and peaks off of the outer atrike point (fis. 12). The density prodile, on the other hand, is peaked very near the separatrix outer atrike point to maintain the peak heat flux magnitude near the separatrix, which reaules in a peak density a factor of 7 higher than the measured value.

It is possible that the inaccuracies in computation of the radial plasma transport introduced by the use of the non-orthogonal grid resulted in a diatortion of radial profiles at the plate. One method to teat this theory is to generate and utilize a grid which reatricts the non-orthogonal region to the last row of radial cells along the outer plate, rather than 
spreading out the non-orthogonality up to the x-point. Presently an estimate of the inaccuracy in radial transport resulting from non-orthogonality is not attempted.

Another possible reason for the discrepancy between the orthogonal and nonorthogonal grid calc slations is that the free parameters in the b2 two-species recycling model (particularly frout) were calibrated only for one input power regime with the nonorthogonal grid and they may need to be scaled with input power and/or transport coefficients. Unfortunately, the best values for the above coefficients probably also depend on the degree of non-orthogonality of the grid. The input parameters used in the benchmarks (previous and present) are tabulated below:

Table 14: Input Parameters for DIII-D Low Power Shot Benchmarks

\begin{tabular}{|c|c|c|c|}
\hline Parameter & 1990 Benchmark & Orthogonal Grid & Non-orthogonal Grid \\
\hline$R_{p}$ & 0.90 & 1.0 & 1.0 \\
\hline$x_{1}^{\prime}, x_{1}^{\prime}, D_{\perp}$ & $1.0,0.2,1.0$ & $2.0,0.5,1.0$ & $2.0,0.5,1.0$ \\
\hline \multicolumn{4}{|l|}{$\left[10^{4} \mathrm{~cm}^{2} / \mathrm{s}\right]$} \\
\hline$n_{e, j} b_{e .}\left[\mathrm{cm}^{-3}\right]$ & $1.0 \mathrm{e} 13$ & $1.5 \mathrm{e} 13$ & $1.5 \mathrm{e} 13$ \\
\hline$T_{e,}, T_{i}$ b.e. $[\mathrm{ev}]$ & 70,70 & 130,130 & 130,130 \\
\hline
\end{tabular}

\subsection{Uncertainties in Experimental Data}

It is relevant to discuss the uncertainties in the data at this point and the implications for the aforementioned benchmark. The divertor tile Langmuir probes should be able to predict the local electron temperature to within $25 \%$ since this parameter is obtained from the slope of the current/voltage trace of the probe. The plasma density is more difficult to compute because information on impurity density and effective probe surface area must be accurate. Hence the density approximation is probably valid to within $50 \%$ for most SOL regimes. Heat flux data from the IRTV is regarded as being very accurate with a probable standard error of only about $25 \%$. Hence the benchmark with the 
two-species models above sought to primarily match the measured electron temperature and heat flux.

One major problem with matching all three measured quantities simultaneously is the accurate selection of energy transmission factors used in the calculation of heat flux flowing into the sheath as a function of density and temperature. As described earlier, the heat flux flowing to the plate is related to the plasma density and temperature by:

$$
q_{b l}^{p l}=\gamma_{i} \Gamma_{i}^{p l} k_{b} T_{i}^{p l}+\gamma_{c} \Gamma_{b}^{p l} k_{b} T_{a}^{p l}
$$

where

$$
\begin{aligned}
& \gamma_{i}=\text { ion energy transmission coefficient } \\
& \gamma_{a}=\text { electron energy transmission coefficient }
\end{aligned}
$$

and all other terms have been defined previously. The energy transmission factor has the following components ${ }^{29}$ for a floating plate:

$$
\gamma_{\text {Lot }}=\gamma_{i}+\gamma_{c}=\frac{2}{1-\gamma_{\text {SEB }}}+\left(e \varphi+\gamma_{\text {com }}+\gamma_{k . e .}\right)\left(1-R_{\text {iE }}\right)
$$

where

$$
\begin{aligned}
& \gamma_{S E E}=\text { secondary electron emission coefficient from the plate } \\
& \frac{2}{1-\gamma_{S E B}}=\text { electron heat flux contribution }-2\left(\gamma_{S B E}=0\right) \\
& e \varphi=\text { sheath potential drop }-2-4 * T \text {. } \\
& \gamma_{\text {conv }}=\text { ion convection contribution } \sim 2.5 \\
& r_{k .}=\text { kinetic energy contribution }-1 \\
& R_{L}=\text { reflected ion energy component }
\end{aligned}
$$

The total energy transmission factor $\left(\gamma_{\text {bor }}\right)$ used in most SOL models lies between 6 and 10, and the value used for all simulations in this analysis is about 7.5. However multiplication of the Langmuir probe particle flux (which density and temperature are obtained from) by the transmission factor of 8 leads to a plate peak heat flux a factor of 2 lower than the heat flux measured by the IRTV, as indicated in fig. 9. Hence it is difficult to match all three measurements simultaneously without adjusting the transmission factor 
used in the b2 code. Discussion of the measurements from the high power input shot will show that use of the same transmission factor of 8 leads to a heat flux computed from Langmuir probe data a factor of 2-3 time higher than the heat flux measured by the IRTV. The discussion above indicates that use of a total transmission factor greater than 8 is acceptable if the secondary electron emission coefficient is greater than 0 , but an energy transmission factor much less than 8 is not consistent with the present understanding of the collisionless sheath unless the ion energy reflection coefficient is high $\left(R_{i E}\right)$. However, analysis of the particle reflection coefficients and reflected energies applicable to the peak electron temperatures computed by the molecular physics model indicates that the total ion energy reflection coefficient above $\left(R_{i E}\right)$ is about $11 \%$, which is anticipated to have a small effect on the energy transmission coefficient.

\subsubsection{High Power Input Shot (10 MW)}

\subsection{Brief Shot Description}

The DIII-D shot selected for this case is 73617. Relevant traces for this shot are given in fig. 13. The plasma current $\left(I_{p}\right)$ is maintained at 1.0 MA and the neutral beam input varies between 0 and $10 \mathrm{MW}$. The onset of $\mathrm{H}$-mode confinement occurs at $t=2000 \mathrm{~ms}$, accompanied by the usual increase in line-averaged density and reduction in divertor h-alpha light. However the confinement properties of this H-mode shot are different from the previous shot in that frequent ELMs occur, indicated by the rise in the divertor $h$-alpha light (and radiated power) after the initial drop at $t=2000 \mathrm{~ms}$. Plasma density, temperature, and heat flux data on the outboard divertor plate from Langmuir probes and the IRTV is presented at $t=3000 \mathrm{~ms}$ in fig. $14-16$, prior to the time the $x$-point is swept across the divertor floor ( $t=3150 \mathrm{~ms}$ to $\mathrm{t}=3450 \mathrm{~ms}$, indicated by the $r x p$ trace).

\subsubsection{Previous Benchmark Results}

In an effort to avoid scaling of input parameters, the same input parameters used in the low power shot benchmark ${ }^{13}$ described above were used for the simulation of the data of this shot ${ }^{14}$. The 2-D grid used in this simulation was an EFITD-based grid. 


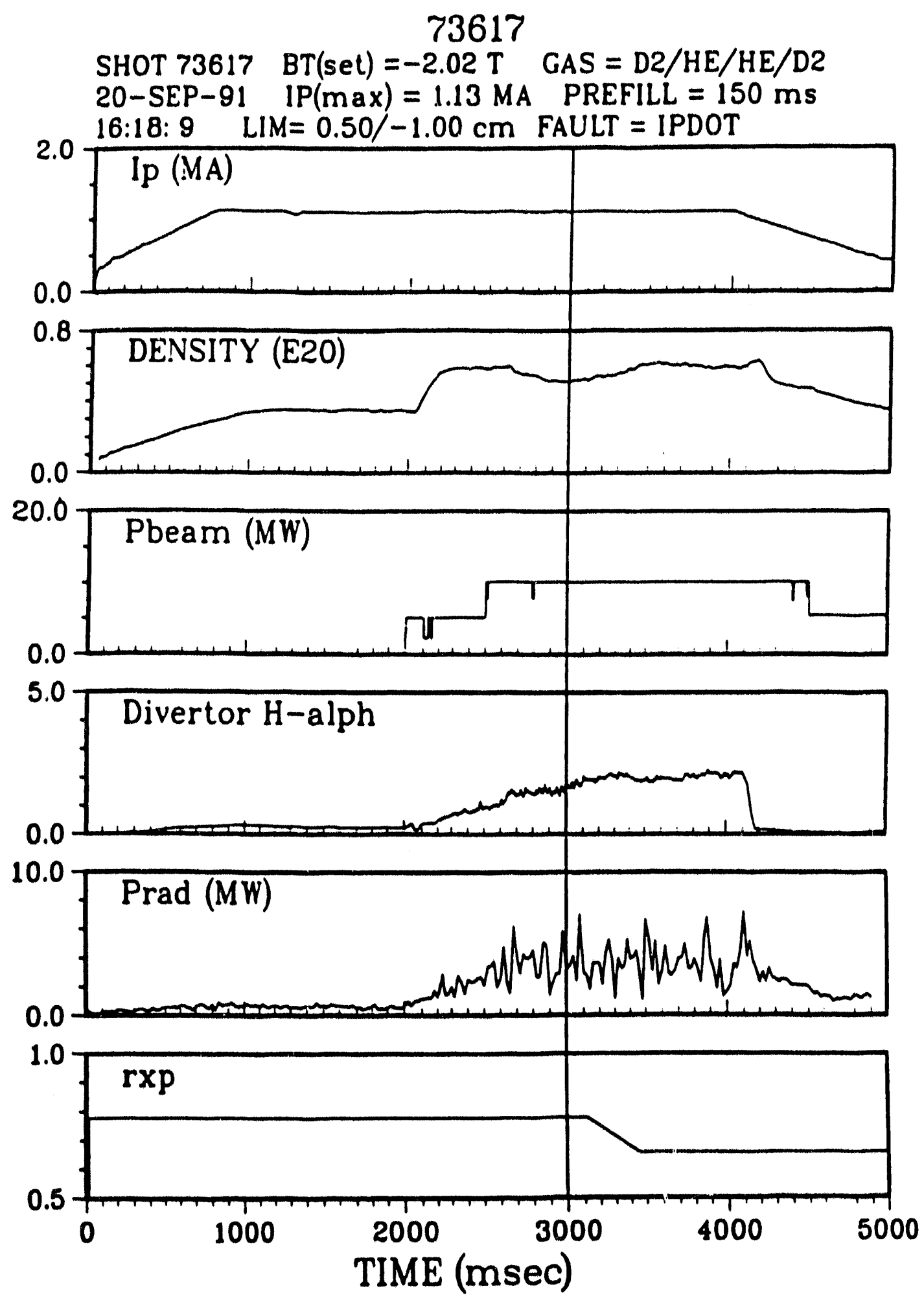

Figure 13: Relevant characteristics 14 of shot 73617 


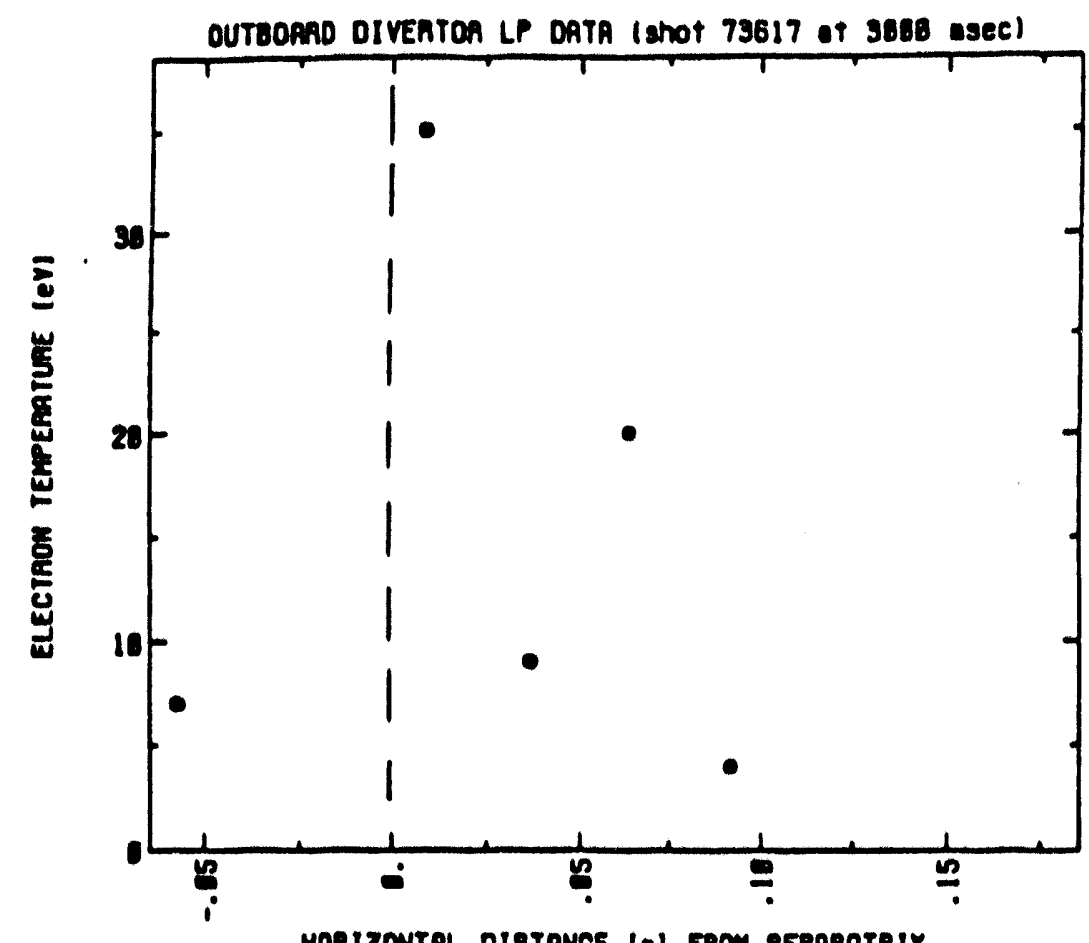

hOAIzONTAL DIstanCE IUI FROM SEPARATAIX

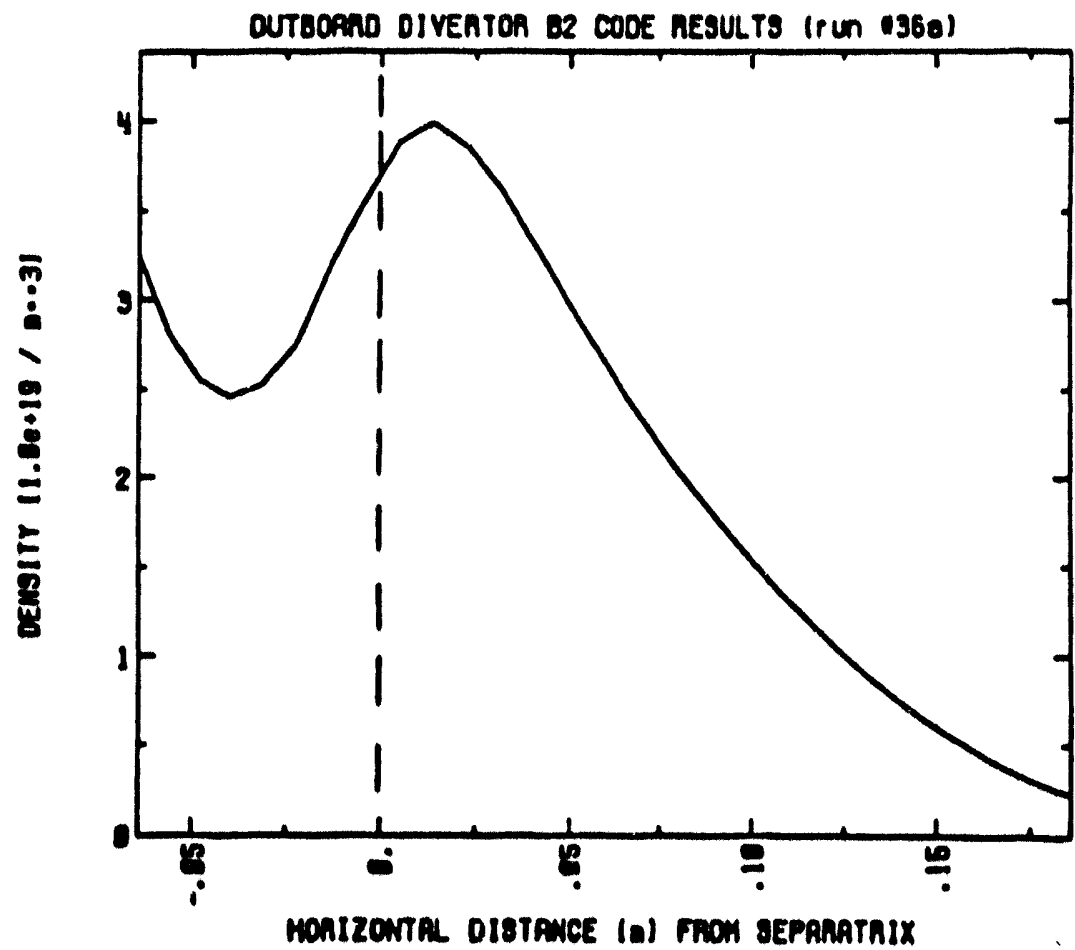

Figure 14: 1991 benchmark ${ }^{14}$ measured (top graph) and computed density profiles (bottom graph) along the outboard divertor plate for shot 73617 

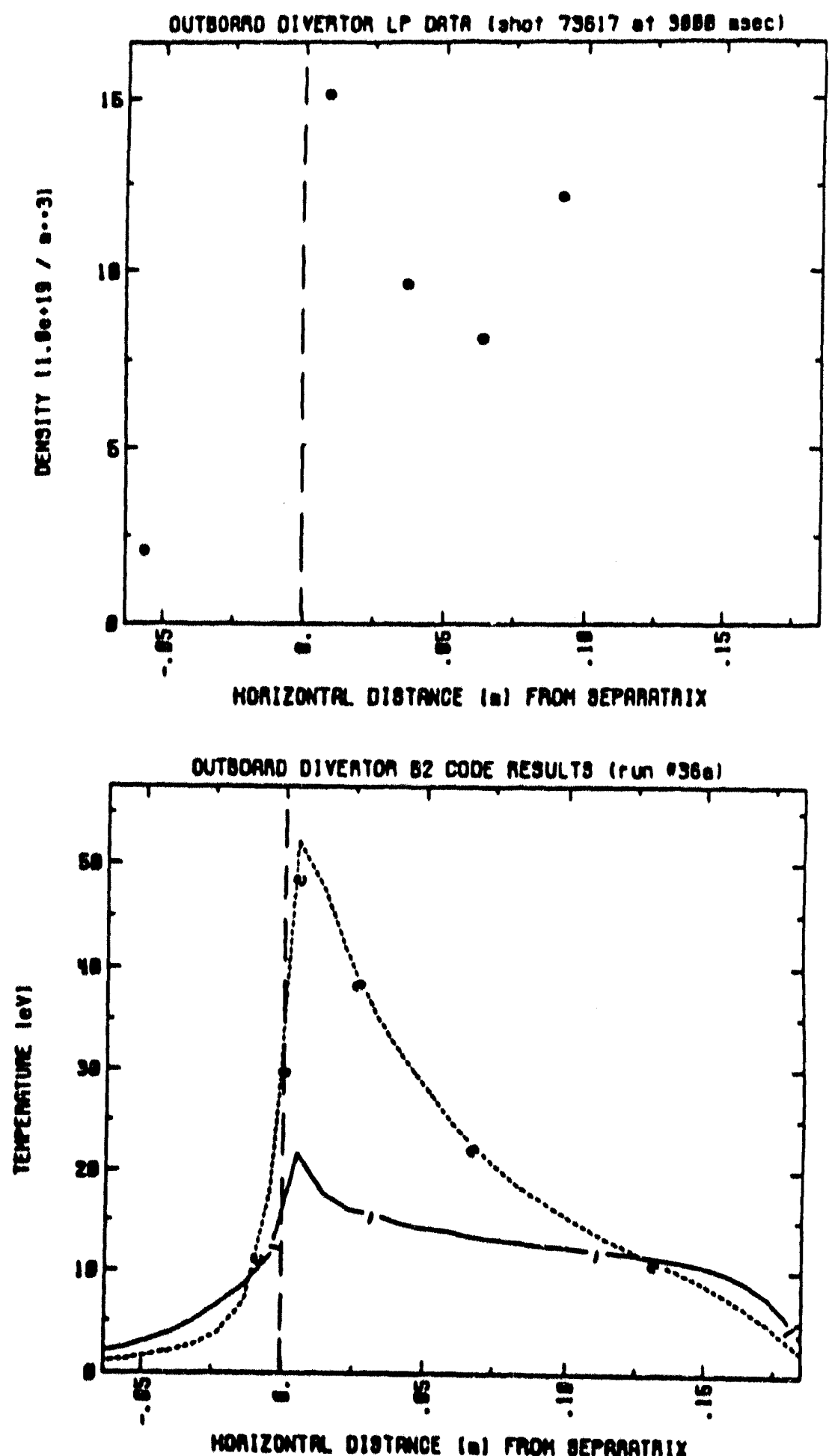

Figure 15: 1991 benchmark 14 measured and computed temperature profiles along the outboard divertor plate for shot 73617. The subscript " $\mathrm{j}$ " is for ions, and "e" for electrons. 

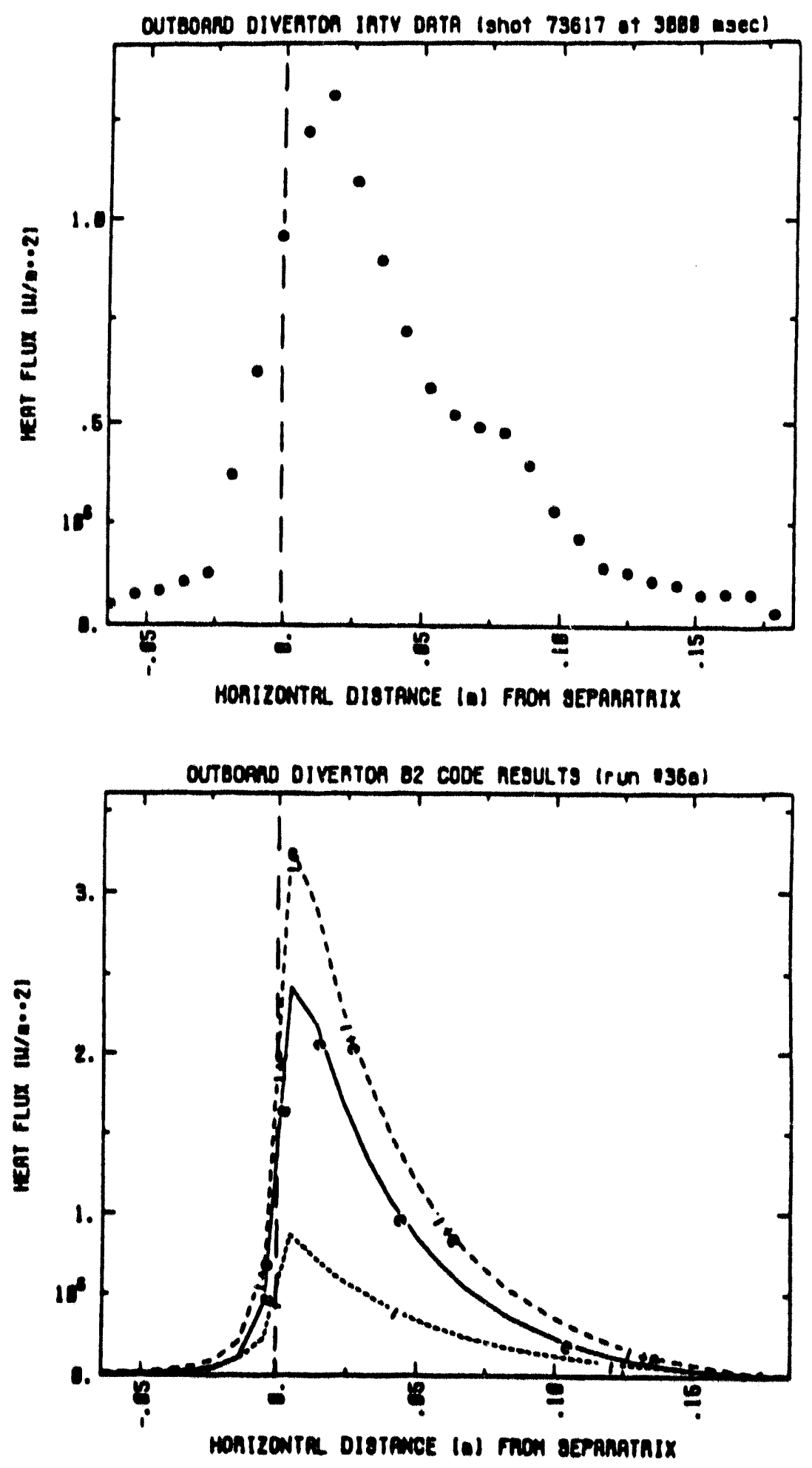

Figure 16: 1991 benchmark14 measured and computed heat flux profiles along the outboard divertor plate for shot 73617. The subscript " $\mathrm{in}$ is for ions, and "e" for electrons. 
Plasma profiles computed for this shot are presented in fig. 14-16, along with the measured quantities. As seen in the aforementioned figures, the peak divertor plate plasma density measured by the Langmuir probes is almost a factor of 4 higher than the computed density, while the measured peak electron temperature is $30 \%$ lower than the computed temperature. It is probable that a higher recycling regime existed in this shot than shot 66821 , and that use of a higher recycling coefficient in the simulation would have lead to a better fit to the data.

\subsection{New Benchmark Results}

As in the low power shot benchmark with the two-species model, simulation of the data from shot 73617 was done with both an orthogonal and non-orthogonal EFITDbased grid. Each simulation is discussed below.

The 2-D grid used here was based on EFITD calculations for shot 73617 at $t=3000 \mathrm{~ms}$. Plasma profiles along the outboard divertor plate are depicted below:

el Pleame Donelty ve. Plate Redlus

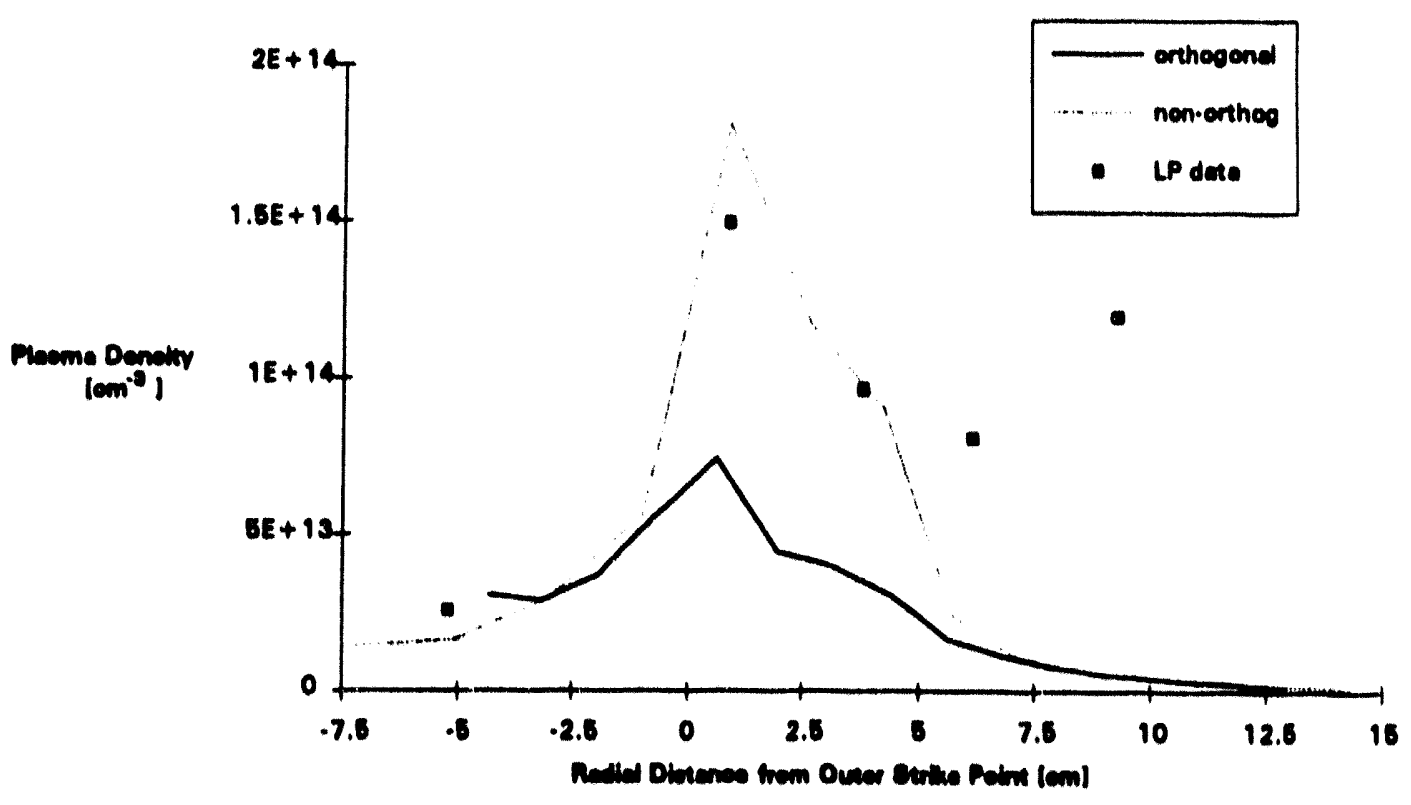


b) Ebotron Temperature ve. Plate Aedius

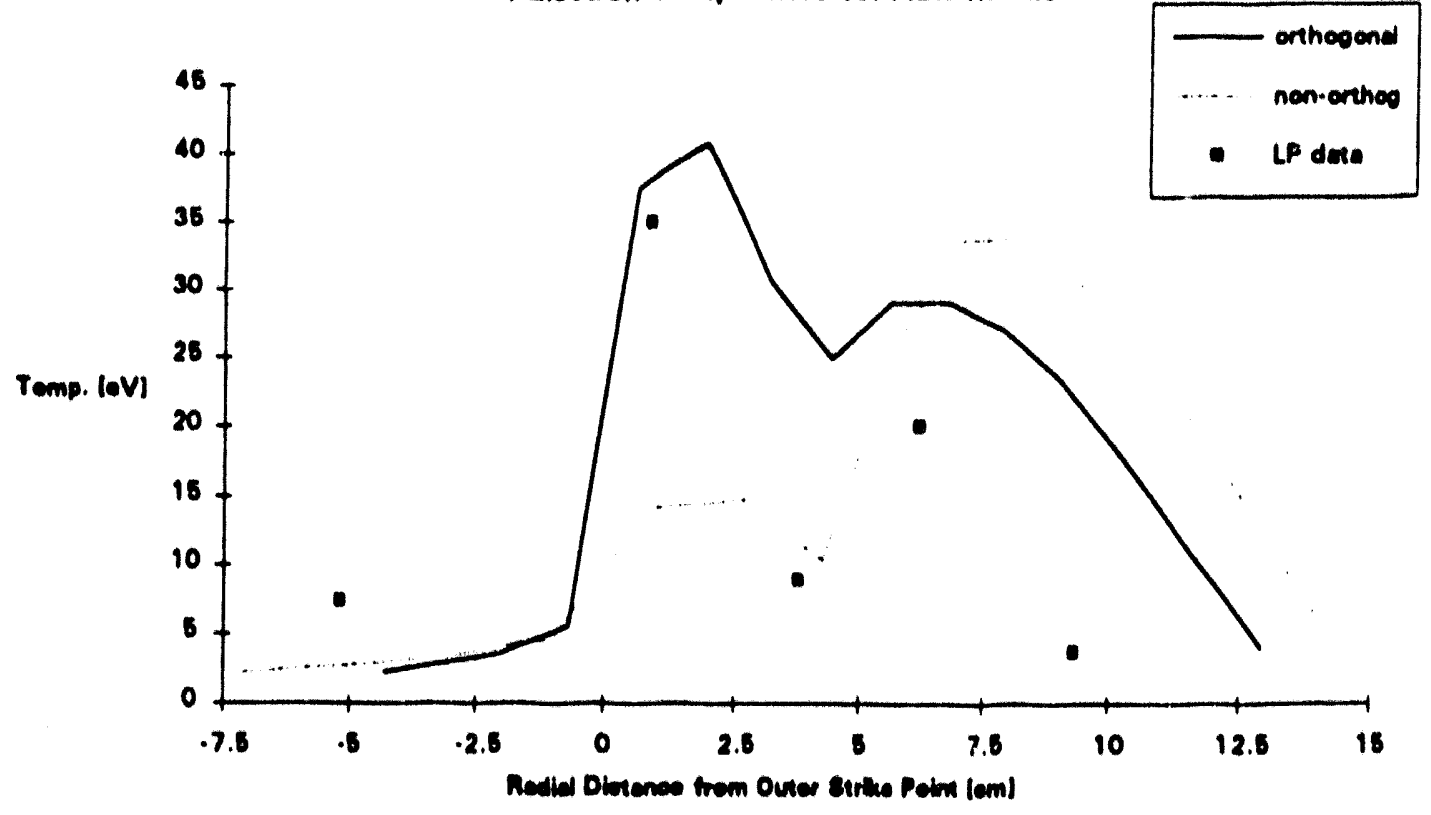

of Piote Howe Flux ve. Redlus

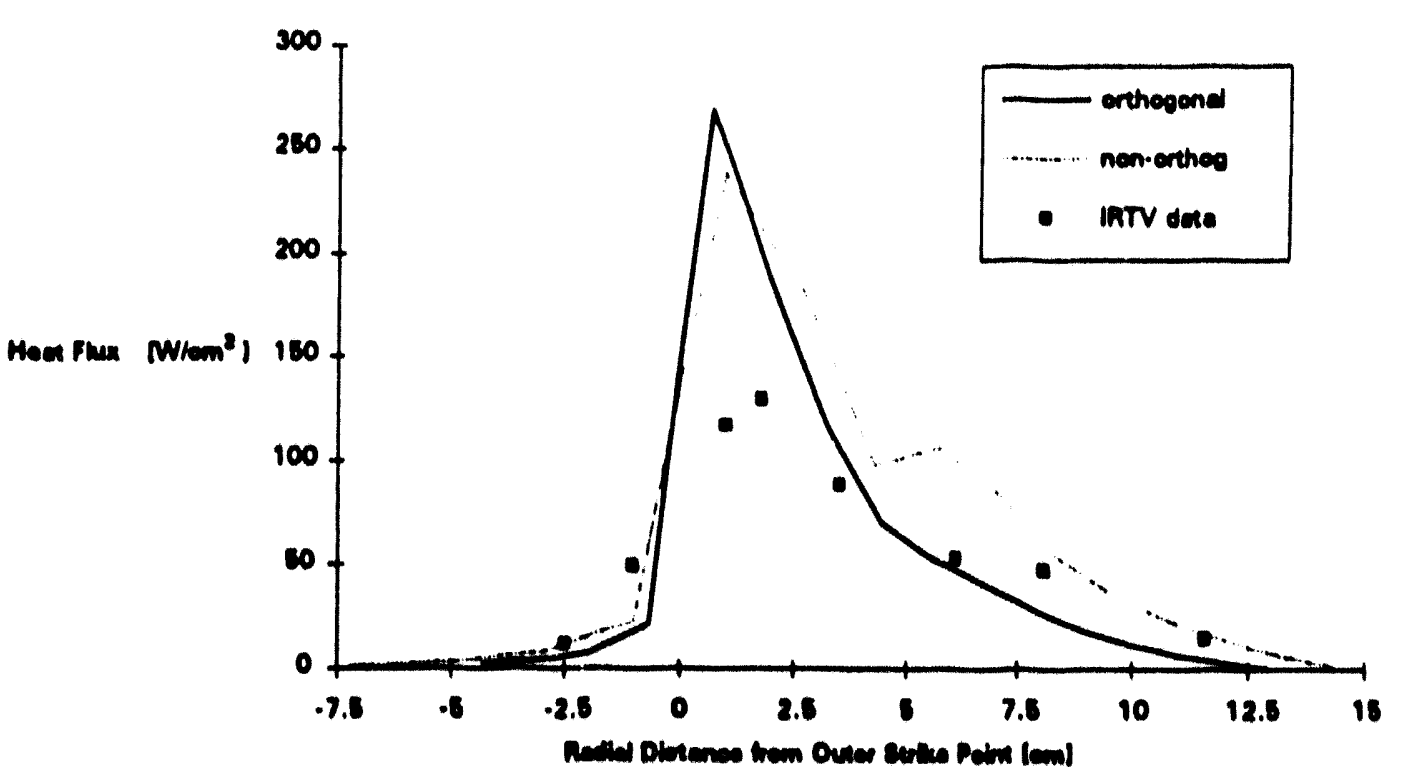

Figure 17: Comparisons of (a)plasma density, (b)electron temperature, and (c)plasma heat flux profiles along the outboard divertor plate between experimental data fom the high power shot and both the orthogonal and non-orthogonal grid simulations 
The computed peak heat flux on the outboard plate is a factor of 2 higher than the reasured value and the peak density is a factor of 2 lower; possible reasons for the discrepancy are discussed in the next section. Of particular interest is the double-hump structure in electron temperature, which is also detected by the Langmuir probe. The probable reason for the double-hump structure is that the recycling particle source and associated electron energy sink peaks off the separatrix, which leads to a depressed value of electron temperature in the proximity of the recycling peak. The double-hump temperature structure is probably a real phenomenon and not a numerical or measurement error.

Table 15: Input Parameters for DIII-D High Power Shot Benchmarks

\begin{tabular}{|c|c|c|c|}
\hline Parameter & 1991 Benchmark & Orthogonal Grid & Non-orthogonal Grid \\
\hline$R_{p}$ & 0.90 & 1.0 & 1.0 \\
\hline$x_{1}, x_{1}, D_{1}$ & $1.0,0.2,1.0$ & $2.0,0.5,1.0$ & $2.0,0.5,1.0$ \\
\hline \multicolumn{4}{|l|}{$\left[10^{4} \mathrm{~cm}^{2} / \mathrm{s}\right]$} \\
\hline$n_{e, ~} b_{e .}\left[\mathrm{cm}^{-3}\right]$ & $2.5 \mathrm{e} 13$ & 3.0013 & $3.0 \mathrm{e} 13$ \\
\hline$T_{\text {e. }} T_{1}$ b.e [ov] & 150,150 & 200,200 & 200,200 \\
\hline
\end{tabular}

The same magnetic flux data used above was used to generate a non-orthogonal grid for this simulation. As in the orthogonal grid simulation, the peak heat flux in the simulation is higher than the measured value and the peak density is lower than the measured value. The double-hump structure in electron temperature is even more pronounced in this simulation than in the previous simulation and the magnitude of the valley appears very similar to the measured profile, although the electron temperature peaks off of the separatrix in the simulation and near the separatrix in the experiment. Inaccuracies in the radial transport calculation introduced by the non-orthogonal grid approximation appear to have a smaller effect on the equilibrium solution, probably 
because the poloidal component of the parallel transport is even more dominant over the radial transport in this high temperature regime (see chapter 3 for classical scaling of transport coefficients). The input parameters used for the high power benchmarks are tabulated above.

\subsubsection{Uncertainties in Experimental Data}

The general uncertainties pertaining to Langmuir probe and IRTV measurements discussed in the low power benchmark section apply here also. In addition, there seemingly exists an inconsistency between the IRTV data and Langmuir probe data for high power shots on the DIII-D tokamak. The poloidal heat flux on the outboard plate can be extrapolated from the Langmuir probe measurements, assuming a value for the sheath energy transmission factor. The recommended value for the total transmission factor is between 6 and 10, as stated previously. However the heat flux extrapolated from the Langmuir probe measurement assuming a transmission factor of 8 is approximately a factor of 2-3 higher than the heat flux measured by the IRTV. Provided that the measurements made by the diagnostics are free of calculation errors, the sheath energy transmission coefficient needed to satisfy both measurements is about 3, which is far lower than predicted or allowed by eqn. (4.6). Hence other explanations must be considered to explain this apparent inconsistency.

One significant difference between the high power shot and the low power shot is the presence of the biasing ring, which was installed after shot 66821 . The additional recycling off the biasing ring could have generated impurities which then contributed to the particle flux measured by the Langmuir probes. In this case the electron temperature estimation from the Langmuir probe data might be unaffected, but the computed plasma density would be higher than the actual fuel ion density.

This unusual behavior of the DIII-D divertor energy transmission factor has been documented 40,41 . One of these theories 41 discusses the viability of a collisionless sheath on the DIII-D tokamak, and concludes that the ion charge exchange mean-free-time 
inside the sheath is less than or on the order of the ion transit time through the sheath to the plate. If a large fraction of ions were actually charge exchanging inside the sheath, the ion energy transmission through the sheath would drop significantly since the energetic ions would be replaced by cold charge-exchanged ions. This effect would be more prominent in recycling regimes where neutral density near the plate is very high, i.e. in high power and high recycling regimes, and would help explain the apparent difference in the energy transmission factors required to reconcile Langmuir probe and IRTV measurements.

\subsection{Results of the Tore Supra Simulation}

Oniy one coupled b2/DEGAS simulation was done for the Tore Supra tokamak. The original intent of the Tore Supra modeling effort was to use the b2/DEGAS coupled code set to moviel a series of hydrogen (not deuterium) discharges from May 1990. Hence the radial transport coefficients and boundary condition density and temperatures were chosen as best estimates to represent the data from shots 3126-3130. Multiple simulations with variation of input parameters was not done in the Tore Supra analysis as it was in the DIII-D analysis because of the large amount of time required per DEGAS run (about 60 Cray-II CPU minutes). The variation of input parameters for this analysis should be completed after several new variance reduction techniques are tested and perfected in the DEGAS code.

The pump-limiter is located on the outer midplane of the device and is a buttontype limiter with finite poloidal and toroidal extent (fig. 4 in chapter 3). The position of the

duct in the pump-limiter throat leading to the pumping chamber is shown in fig. 18. Pumping is switched on and off by opening and closing of the pump flaps.

\subsubsection{Shot Description and Available Diagnostic Data}

Shot 3127 had ohmic input power of $1.8 \mathrm{MW}$ and active pumping, while shot 3126 had the same conditions with non-active pumping. Approximately 1/3 of the ohmic input power is assumed to be radiated, and the rest of the power (1.2 MW) is assumed to 
have been deposited at the limiter. Measurements of electron temperature and plasma density for shots 3126-3127 were provided by Langmuir probes in the throat of the pumplimiter (fig. 18). The probes were used as an asymmetric double probe which yielded an estimate of the radial decay length of the parallel ion flux in the throat. The estimate is based on a two-point measurement and the assumption of an exponential decay profile between those two points. Estimates of these quantities are available on both the electron drift side and the ion drift side of the pump-limiter, and values from both locations are used to compare with computed values from the modeling effort in the following sections.

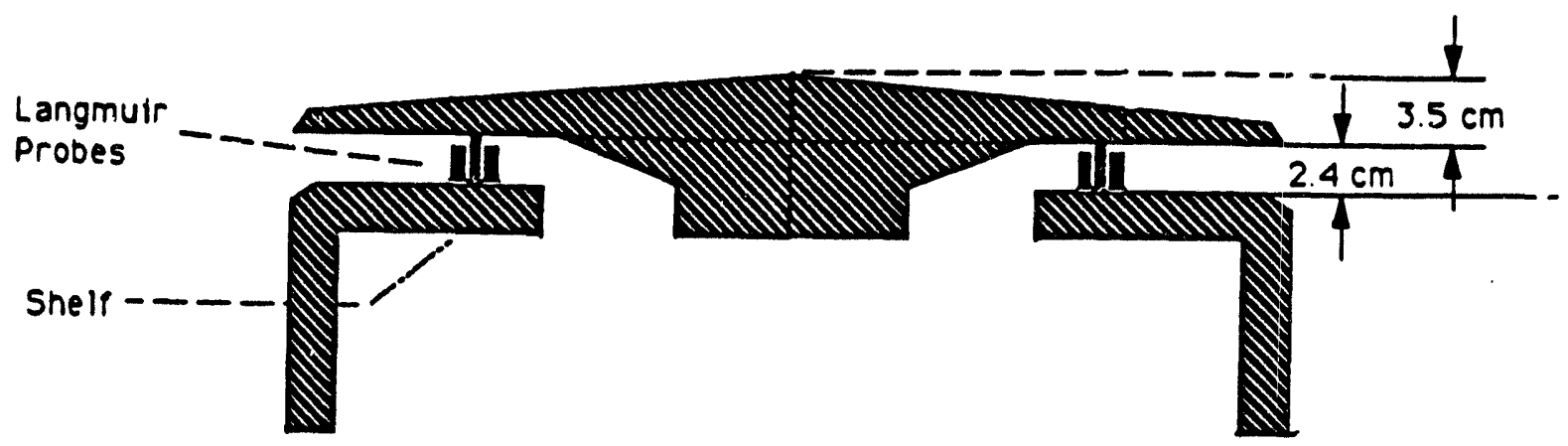

Figure 18: Location of Langmuir probes in pump-limiter throat (courtesy of P. K. Mioduszewski)

A diagnostic which was available on similar shots but not the shot modeled in this analysis is the IRTV camera, from which the heat flux decay length along the limiter plasma-facing surface (from the tangency point to the leading edge) can be obtained. For shots similar to this one, a heat flux decay length $\left(\lambda_{q}^{\text {tad }}\right)$ along the limiter plasma-facing surface of $1.2-1.5 \mathrm{~cm}$ has been measured 42 . Using this measured value of $\lambda_{q}^{\text {hoded }}$, the heat 
flux at the leading edge of the limiter can be estimated by a power balance. Assuming that the heat flux profile along the limiter head is exponential, the power incident on the limiter plasma facing surface is given by :

$$
P_{\text {lim }}=\Delta y \times \int_{0}^{R_{14}} q_{0}^{L \mathrm{CrS}} \exp \left(-r / \lambda_{q}^{\text {mod }}\right) d r
$$

where

$$
\begin{aligned}
& P_{\text {lim }}=\text { total power to the limiter }=1.2 \mathrm{MW} \\
& \Delta y=\text { poloidal width of limiter }=65 \mathrm{~cm} \\
& q_{0}^{\text {LCrS }}=\text { heat flux at the last-closed-flux-surface } \\
& R_{1.0}=\text { radial distance from limiter tangency point to leading edge }=3.5 \mathrm{~cm}
\end{aligned}
$$

The solution to the above equation for the heat flux at the leading edge is:

$$
q_{\text {iat }}^{\text {lea }}=q_{0}^{\text {LCrs }}-\frac{P_{\text {iming }} \times \lambda_{q}^{\text {hod }}}{\Delta y}
$$

This extrapolated heat flux value is the one quoted in the comparison section, and it should be very close in magnitude to the peak heat flux on the neutralizer plate for cases with active pumping.

\subsection{2. b2/DEGAS simulation}

As mentioned previously, the x-direction in the fluid equations of the b2 code for the Tore Supra simulation represents the toroidal direction, which is chosen because variation of plasma parameters is more likely in the toroidal plane than in the poloidal plane far away from the pump-limiter. The y-direction represents the radial direction, as in the DIII-D simulations. Because the limiter is localized poloidally, variation of plasma parameters in the poloidal direction occurs only near the limiter itself. To accurately model a limiter which has neither poloidal nor toroidal symmetry with expected variation of plasma parameters in all three directions requires a 3-D simulation. However the dominance of parallel transport in the SOL for limiter discharges suggests that :-2-D simulation (radial and parallel) should be a good approximation. 


\subsubsection{Grids and Modeled Region}

The region $A B C D E$ in fig. 4 (chapter 3 ) represents the physical domain of the calculation. The semi-circular arc in the toroidal view above is approximated as a rectangle with approximately uniform radial thickness and toroidal length. The tilt of the neutralizer plate in the throat is neglected, i.e. the neutralizer plate is modeled normal to the toroidal direction. The total magnetic field is constant $(2.0 \mathrm{~T})$, and the toroidal component is assumed to he $1.8 \mathrm{~T}$. The toroidal length $\left(l_{c}^{\infty}\right)$ of the modeled region (distance from stagnation boundary to neutralizer plate) is given by 43 :

$$
l_{c}^{\phi}=\pi^{*} R_{m}^{*} q_{\text {edge }}-40 \mathrm{~m}
$$

where

$$
\begin{aligned}
& R_{m}=R_{\text {maro }}+R_{\text {min or }}=2.37 \mathrm{~m}+0.75 \mathrm{~m}=3.12 \mathrm{~m} \\
& q_{\text {edore }}=\text { safety factor at the edge }-4 \text { (similar shots) }
\end{aligned}
$$

The Cartesian grid used in the simulation utilizes a telescoping mesh (fig. 19); 18 of the 32 grid points in the toroidal direction are placed outside the pump-limiter throat and the rest are placed inside the throat. The distribution of the 24 radial grid points is concentrated on the inner boundary side (under the limiter head) because the sharpest radial gradients occur along that boundary (i.e. away from the assumed first wall). The radial width of the SOL corresponds to the approximate width of the pump-limiter throat of $2.5 \mathrm{~cm}$ (fig. 18).

As mentioned in chapter 3, the meshes used in the b2 and DEGAS codes were quite different in the Tore Supra simulation. The DEGAS mesh extends down from the duct in the pur p-limiter throat to the pumping chamber. This extension is necessary to properly compute neutral pressure within the pumping chamber and the particle removal efficiency of the pump. The domain over which the DEGAS code needs plasma parameter profiles and supplies source terms lies entirely in the pump-limiter throat for this simulation. The resolution of the DEGAS plasma grid is necessarily more coarse (6 X 5) than the b2 grid ( $32 \times 24)$ to insure small statistical uncertainties in the computed source terms. The source terms computed by the DEGAS code on the DEGAS grid sie 
extrapolated onto the b2 grid as described in the previous chapter, and the plasma profiles from the b2 calculation are averaged over the b2 cells which correspond to the DEGAS cells.

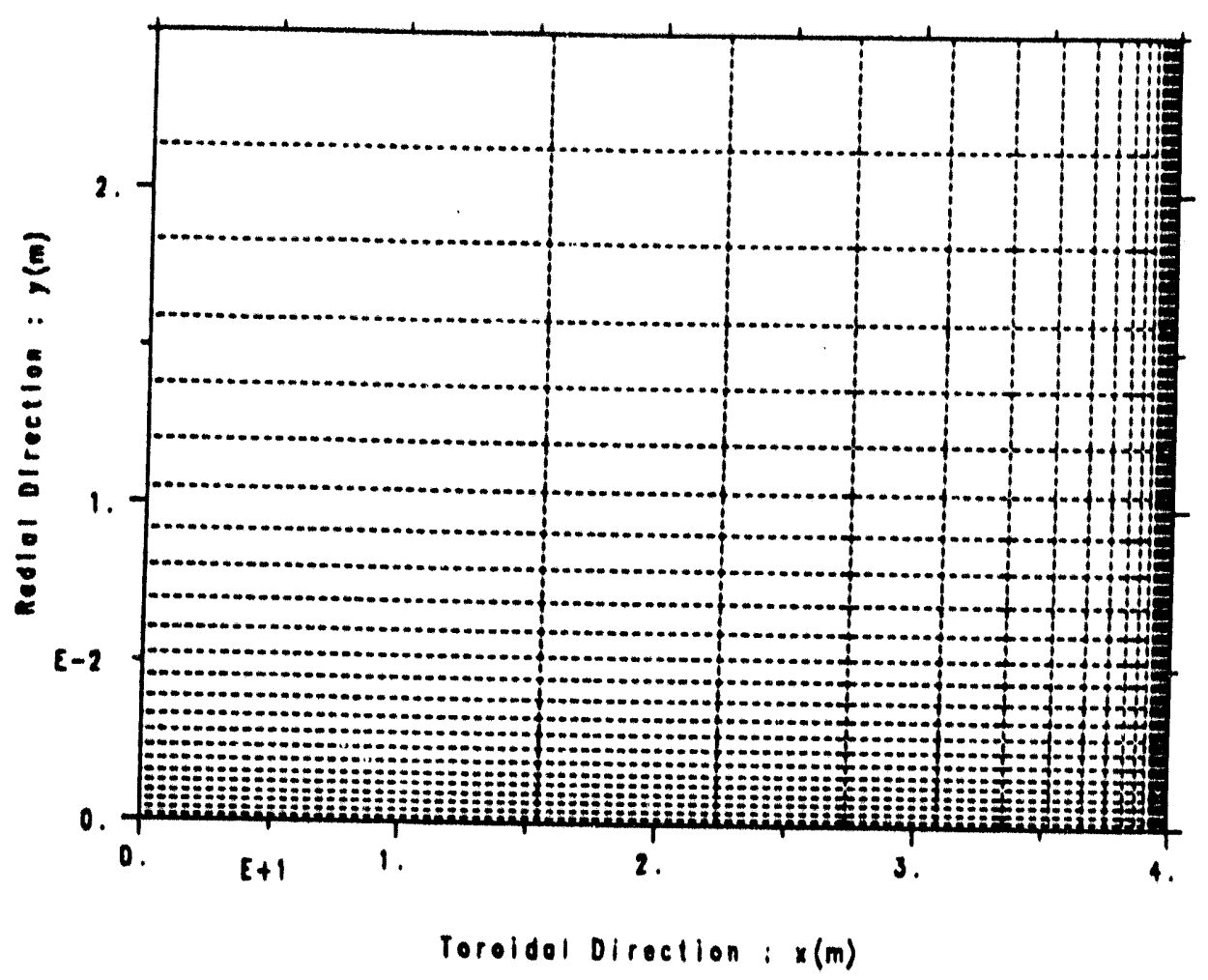

Figure 19: 2-D telescoping mesh used in the Tore Supra analysis

\subsubsection{Input Parameters and Convergence of b2/DEGAS Simulation}

The assumed values of the anomalous radial transport coefficients used in the simulation, as well as the plasma density and temperatures specified along the inner boundary (edge $A D$ of fig. 4 in chapter 3) are tabulated below: 
Table 16: Input Values in the 62 Code for the Tore Supra Simulation

$$
\begin{gathered}
\text { Parameter } \\
\chi_{\perp}, x_{\perp}, D_{\perp}\left[10^{4} \mathrm{~cm}^{2} / \mathrm{s}\right] \\
n_{e, ~} b_{c .}\left[\mathrm{cm}^{-3}\right] \\
T_{e}, T_{i} b_{\text {b.c. }}[\mathrm{ev}]
\end{gathered}
$$

Value

$3.0,1.0,1.0$

$2.0 \mathrm{e} 12$

35

These values were chosen based on several b2 runs using the "uncalibrated" internal single-species recycling model which attempted to match the experimental data available from the shot. The recycling coefficient for the coupled b2/DEGAS runs is actually computed by the DEGAS code, and the estimate used in the first run (run 0 below) was a guess based on previous DEGAS simulations of the pump-limiter with plasma profiles in the throat held fixed.

Table 17: Convergence Diagnostics for b2/DEGAS Simulation (Tore Supra)

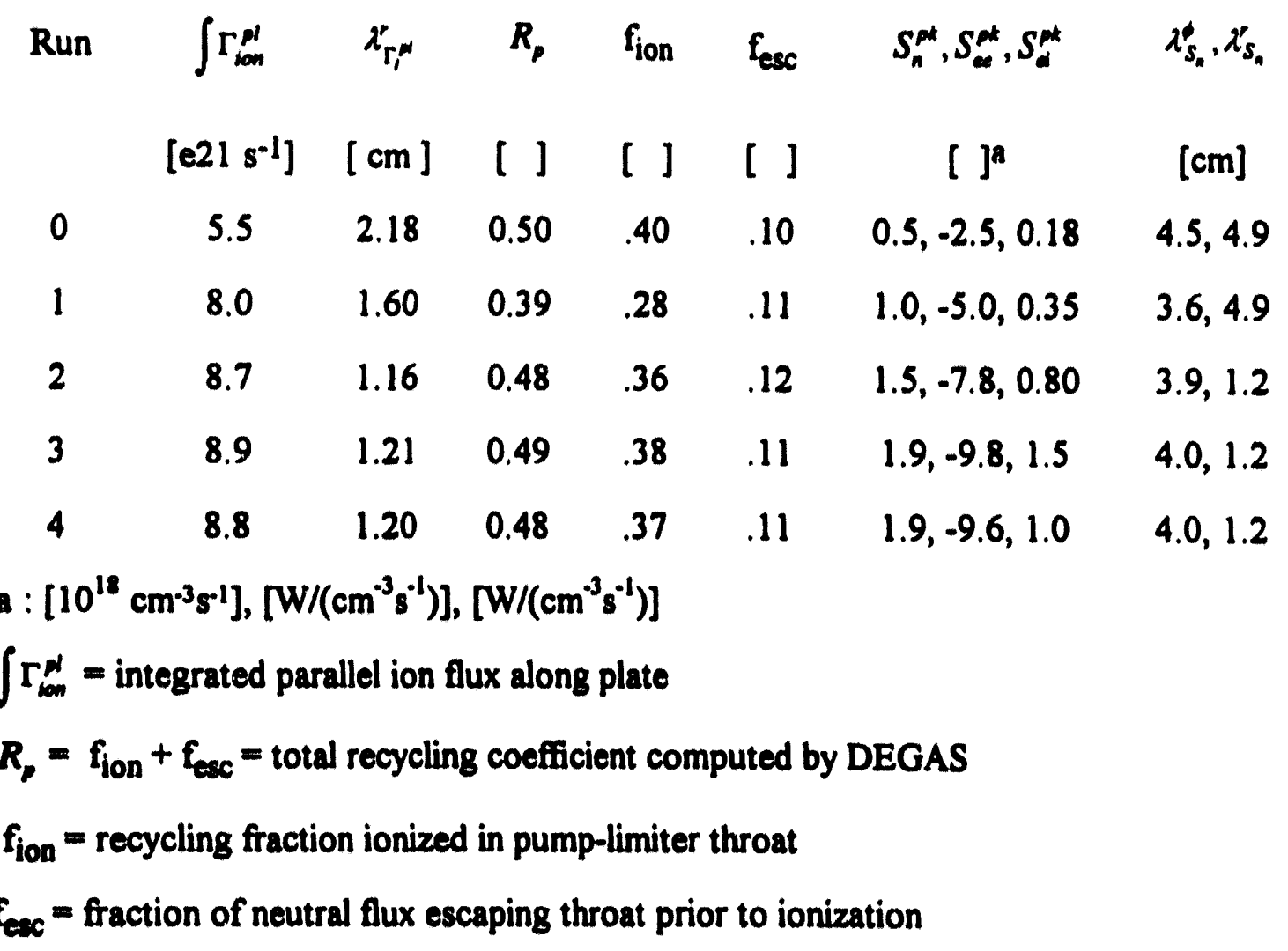


$\lambda_{r_{,}^{\prime \prime}}^{r}=$ radial decay length of parallel ion flux along plate

$S_{n}^{p k}, S_{a}^{p k}, S_{a}^{p k}=$ peak particle, electron energy, and ion energy recycling sources

$\lambda_{s_{a}}, \lambda_{s_{q_{4}}}=$ toroidal and radial decay lengths of recycling particle source term

Four iterations of the b2/DEGAS procedure were required for convergence of the simulation. The reason for the smaller number of b2/DEGAS iterations needed for convergence as compared to the DIII-D simulations is that the Tore Supra SOL is in a lower density regime, which leads to smaller recycling source terms. As indicated in the first chapter, the peak recycling source term varies roughly with the plasma density squared. The table above highlights the large difference between the source terms computed by the b2 code with the "uncalibrated" single-species recycling model (run 0 ) and the coupled code calculation (runs 1-4). The ion energy source peak is the most sensitive of the convergence criteria and also the least important.

\subsubsection{Calibration of b2 Single-Species Recycling Model}

The recycling model used in the calibration procedure is the single-species model internal to b2. As mentioned in chapter 3, the parameters frout, enex(toroidal energy instead of poloidal energy as in the DIII-D simulation), and enerad are adjustable in this model. The theoretical value of frout is 0.5 for an orthogonal plate, which corresponds to half of the neutrals recycled off the plate traveling radially outward and the other half traveling radially inward. The theoretical value of the toroidal and radial energies is more difficult to ascertain since the model attempts to average the energies of desorbed wall molecules and primary reflected neutrals in one species. As mentioned in chapter 3, the default value used in the code is $15 \mathrm{eV}$ for each, corresponding to a neutral temperature of $10 \mathrm{eV}$.

After trial and error, it was determined that values of $0.5 \mathrm{eV}$ and $10 \mathrm{oV}$ for enex and enerad in the b2 single-species model best approximated the source terms computed by the coupled b2/DEGAS model. The parameter frout was fixed at 0.5 . The results from 
calibration of the molecular physics model in the DIIl-D simulation indicate that the addition of a fourth radial sweep, an inward one, would probably reduce the required value of enerad to match the radial distribution of the b2/DEGAS computed source terms. For a detailed discussion of the radial sweep technique used to compute the radial ionization profile of neutrals in the b2 code, see the discussion of recycling models in chapter 3.

Toroidal profiles of plasma density and temperature at a radius of $0.4 \mathrm{~cm}$ outward from the leading edge from both the b2/DEGAS calculation and the calibrated b2 internal model are presented below. The temperature profile is very flat along field lines, and the only part of the density profile that is not flat is in the pump-limiter throat, which extends $16 \mathrm{~cm}$ away from the plate. Because the profiles are plotted up to $12 \mathrm{~m}$ away from the plate, the density drop very near the plate is not noticeable. An enlargement of the toroidal density profiles in the throat is presented in figure 21.

al Plasma Density ve. Toroldel Distenos

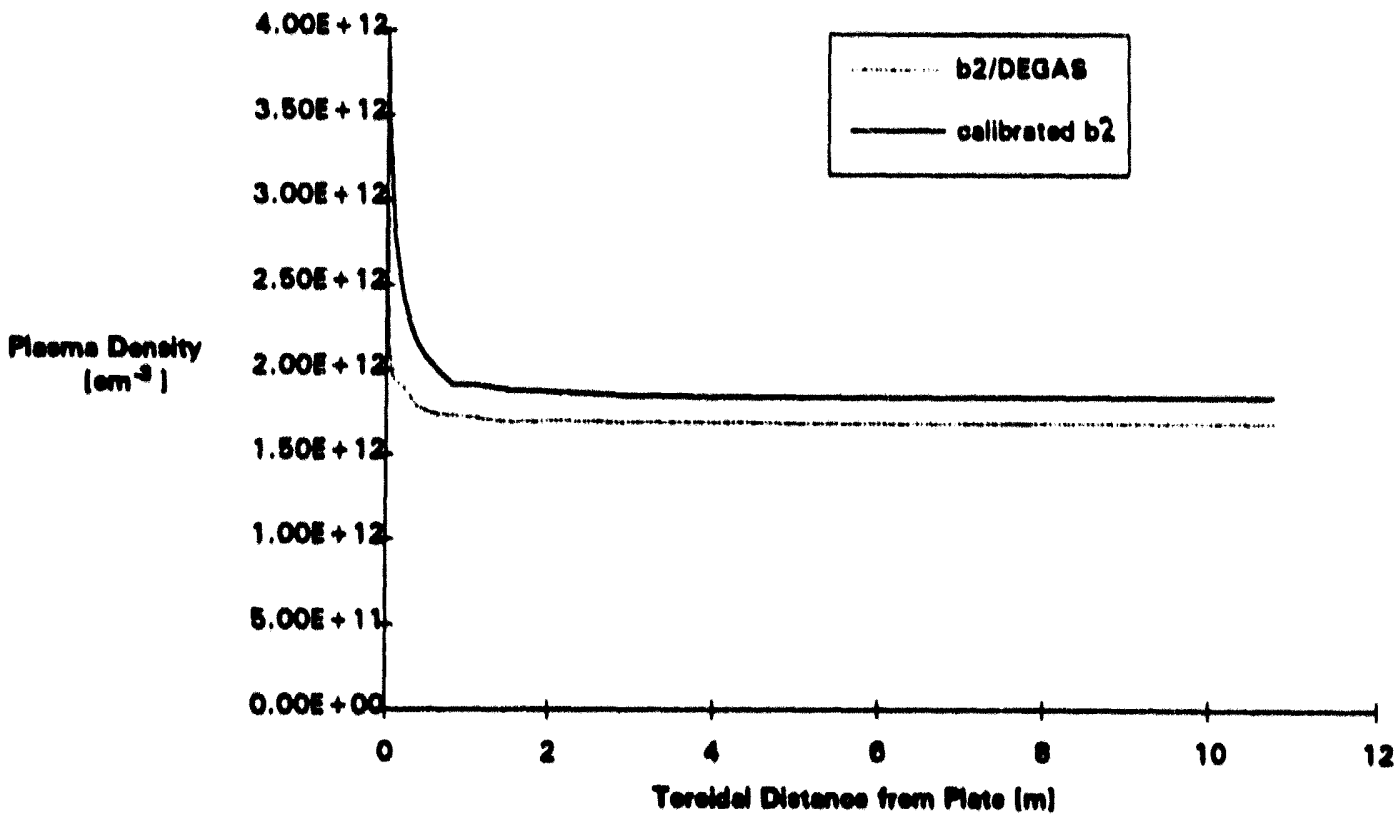


b) Eleotron Temperature ve. Toroldal Oistenes

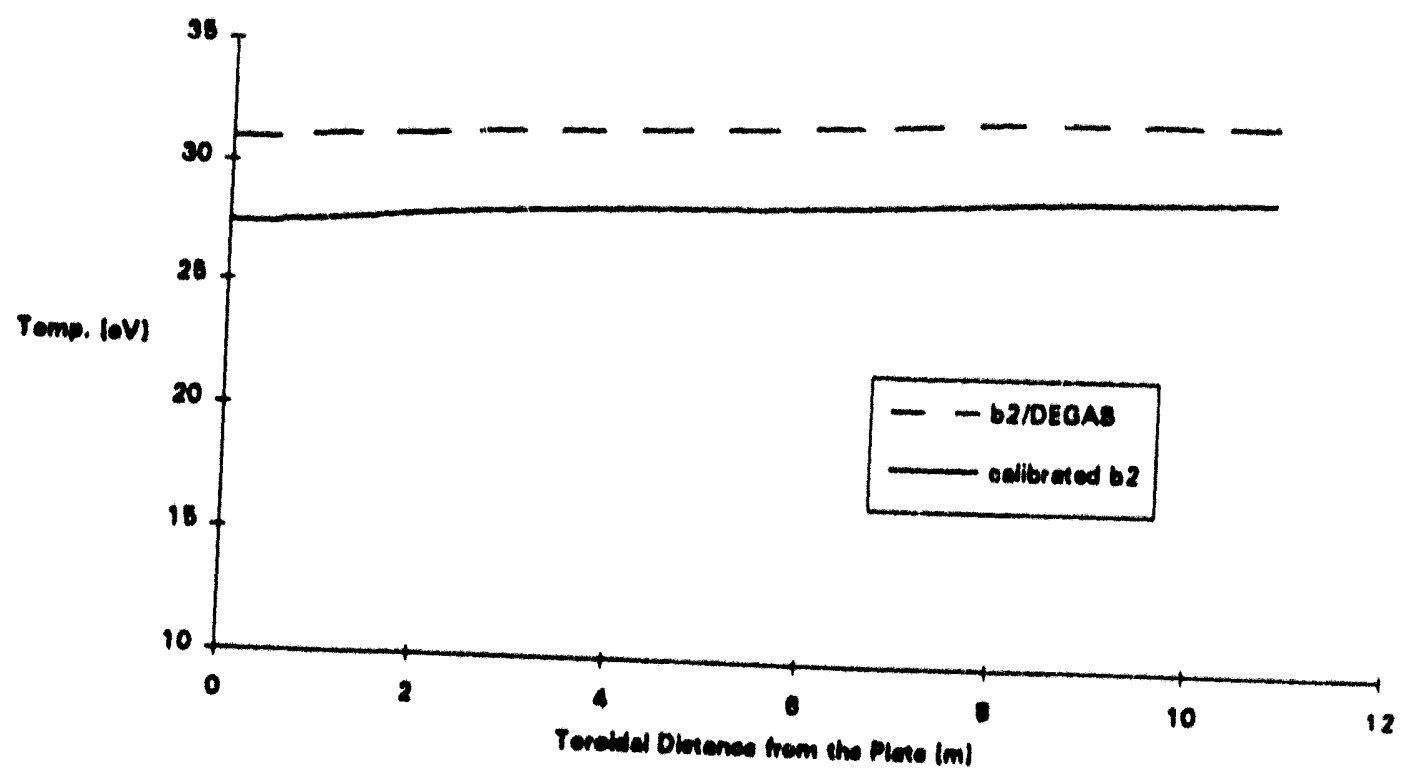

Figure 20: Comparison of toroidal (a)density and (b)temperature profiles for the b2/DEGAS and calibrated b2 runs at a distance $0.4 \mathrm{~cm}$ radially below the limiter head

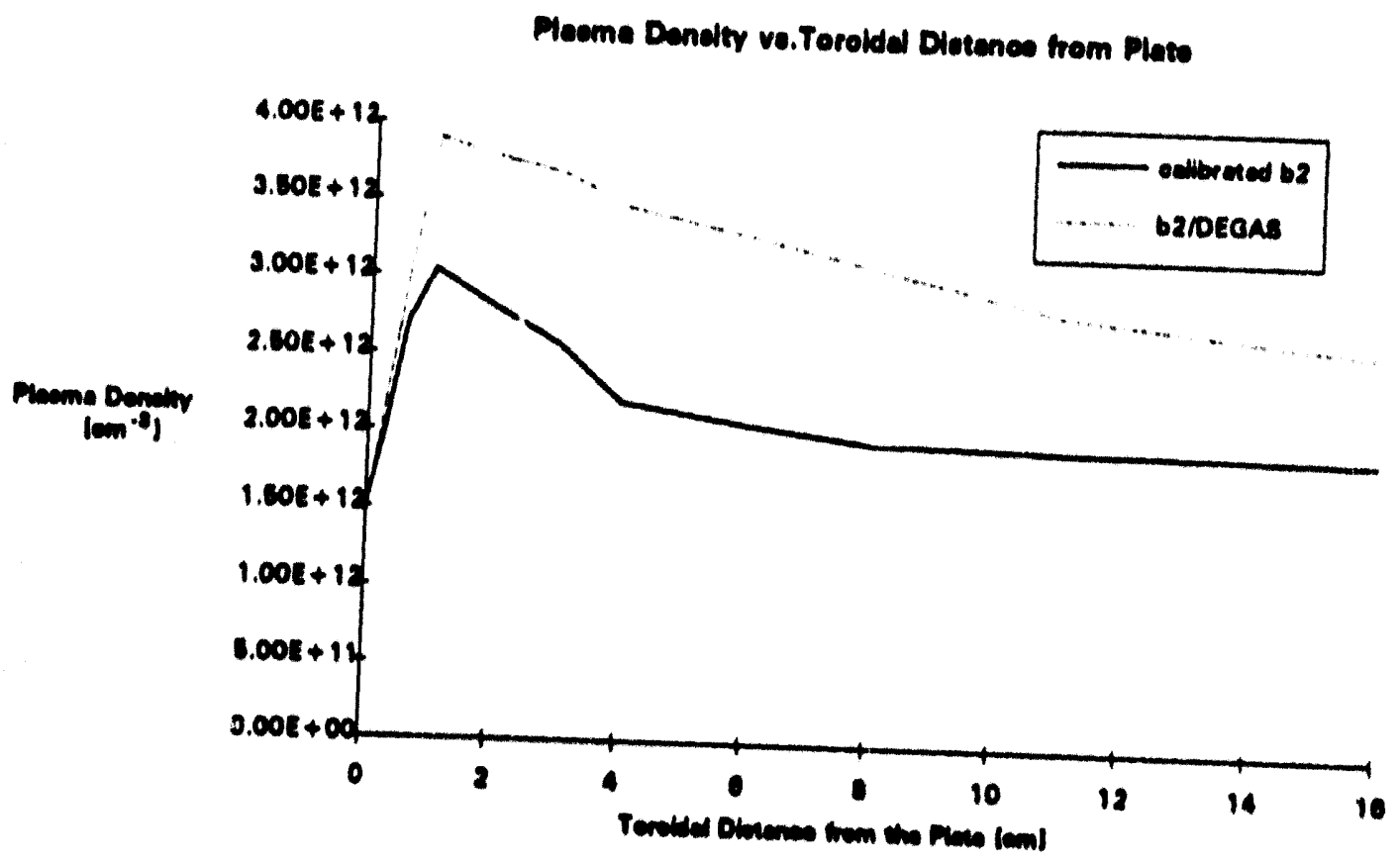

Figure 21: Plasme density profile in the pump-limiter throat for the b2/DEGAS run and the calibrated b2 model 
Radial profiles of plasma density, temperatures, particle flux, and heat flux at the plate are displayed below also. Very little is said about these profiles since no experimental data exists with which to compare the plate profiles. Overall the coupled b2/DEGAS computed profiles are matched fairly well by the calibrated b2 model.

\subsubsection{Comparison with Experimental Data}

Comparisons of the modeling results from the various recycling models with data from shnt 3127 are tabulated below:

Table 18: Comparison of Measured Values and Modeline. Results for Tore Supra

\begin{tabular}{|c|c|c|c|c|}
\hline Parameter & Experiment & b2/DEGAS & b2 (uncalibrated) & b2 (calibrated) \\
\hline$\Gamma_{1}^{L}\left[\mathrm{~cm}^{-2} \mathrm{~g}^{-1}\right]$ & 7.0018 & $7.6 e 18$ & $7.6 \mathrm{e} 18$ & $1.0 e 19$ \\
\hline$\lambda_{r_{1}-\infty}^{r}[\mathrm{~cm}]$ & 2.0 & 1.7 & 2.0 & 1.8 \\
\hline$T_{0}^{L+}[\mathrm{ev}]$ & 36 & 35 & 34 & 35 \\
\hline$n_{6}^{L+\infty}\left[\mathrm{cm}^{-3}\right]$ & $2 e 12$ & $2.1 e 12$ & $2.1 e 12$ & 2.6012 \\
\hline$q_{b i}^{\prime}\left[W / \mathrm{cm}^{-2}\right]$ & $481^{2}$ & 489 & 353 & 421 \\
\hline
\end{tabular}

a) Macme Dendry ve. Radiue

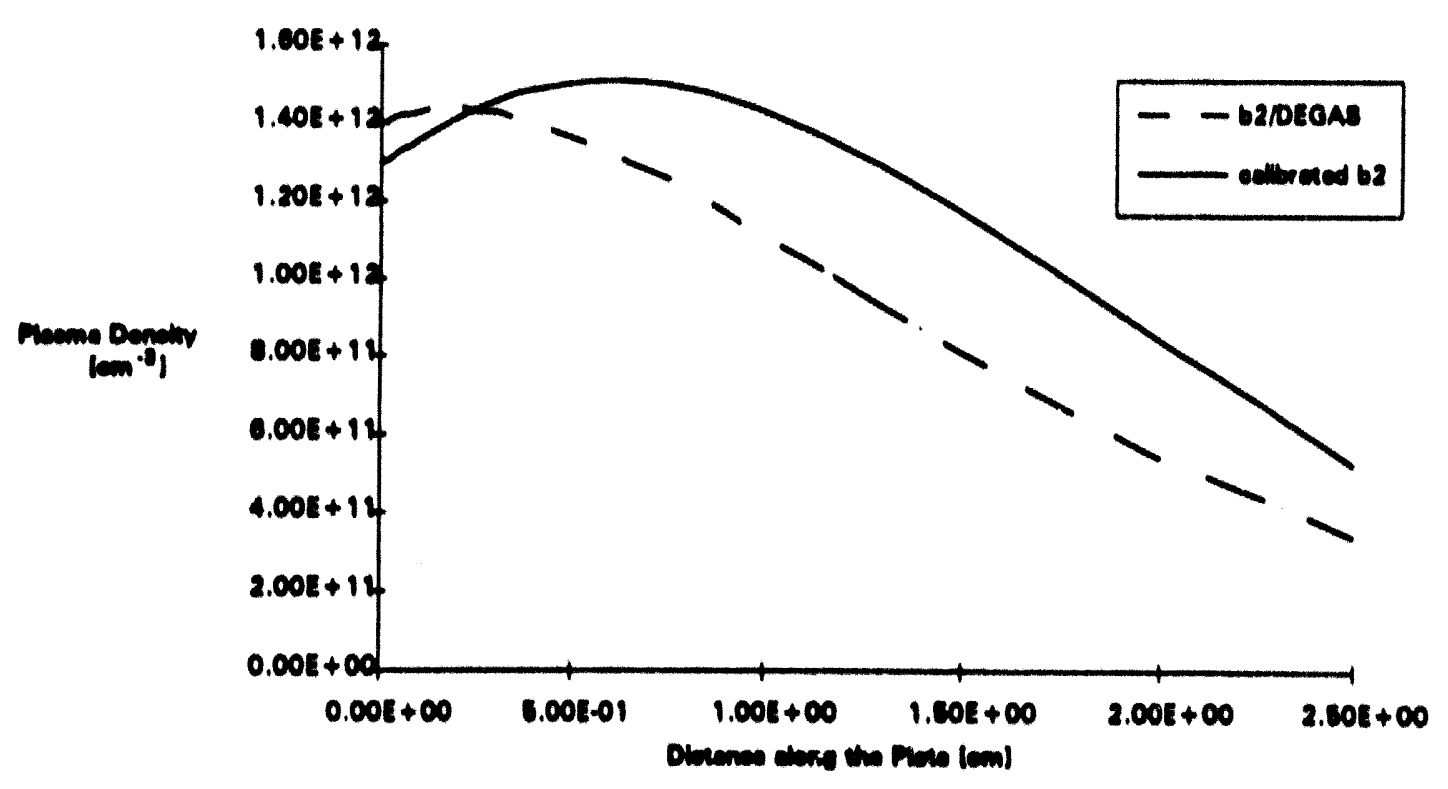


b) Ebetron Temporature ve. Aedlue

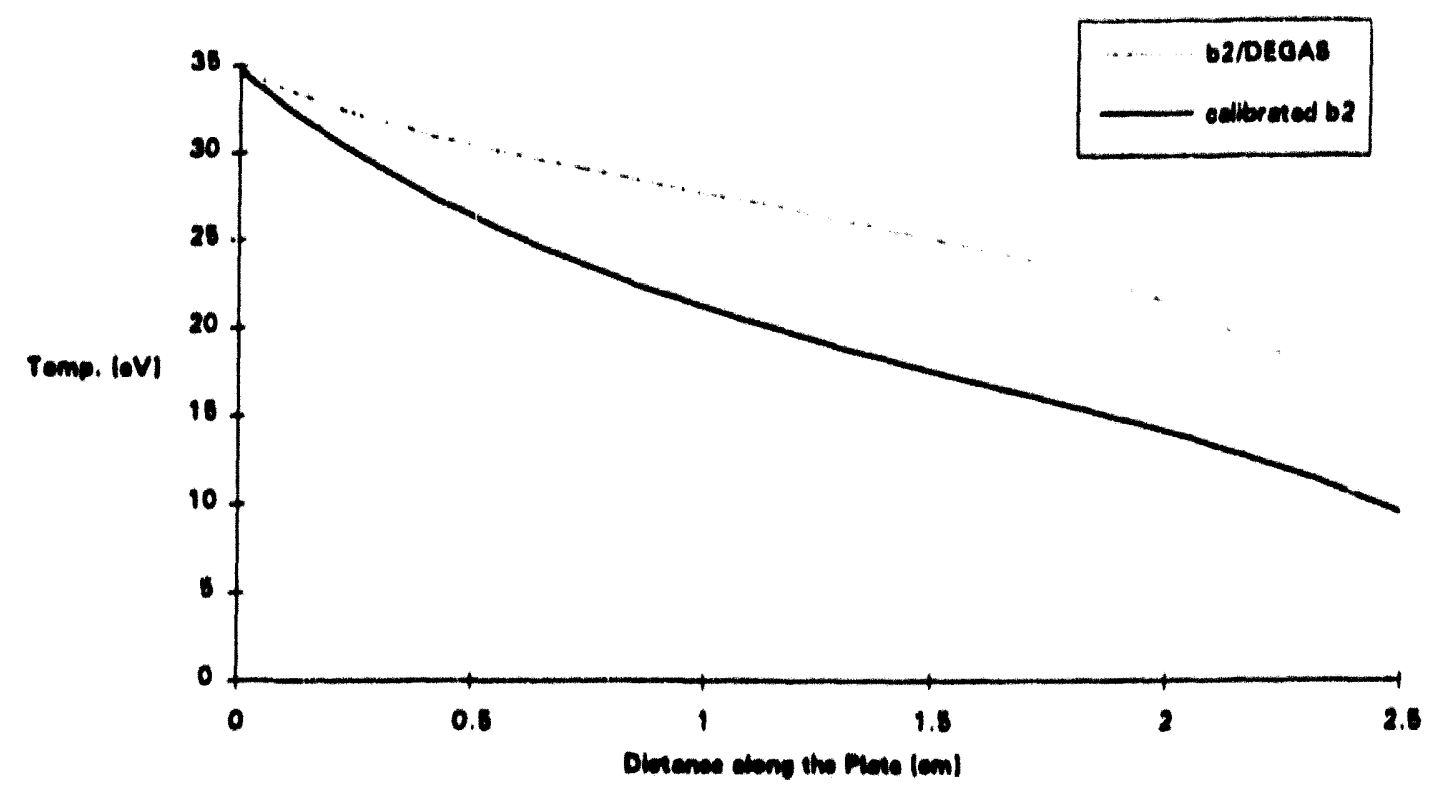

e) Plate Hast Fux vo. Rodlue

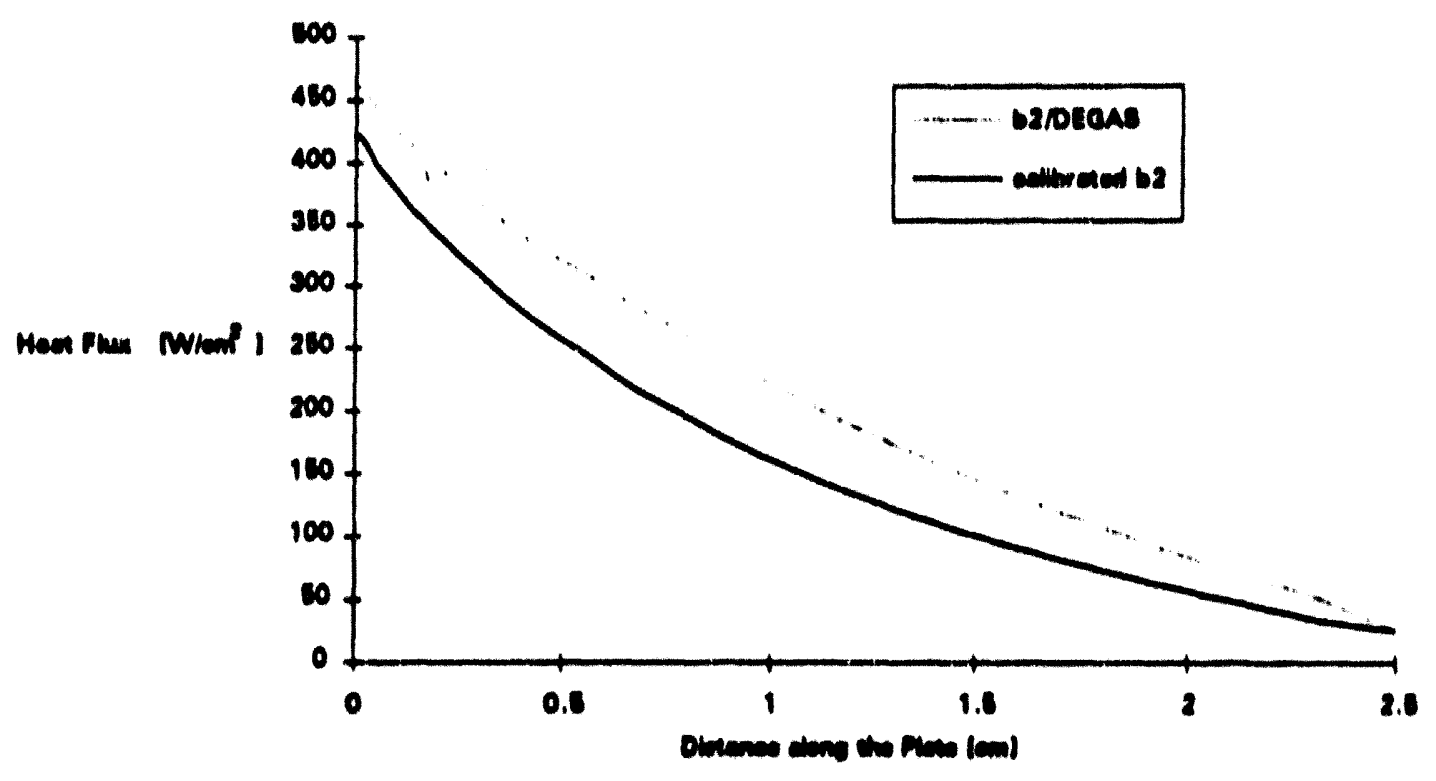

Figure 22: Radial profiles of (a)plasma density, (b)temperature, and (c)heat flux along the plate for the coupled b2/DEGAS simulation and the calibrated b2 model. 
The incalibrated model calculation of the particle flux at the Langmuir probe location is actually better than the calibrated model calculation. The reason for the difference is that the plasma density and ion temperature (not shown) computed by the uncalibrated model is higher than the other two models, leading to a higher computed peak particle flux at the probe. The uncalibrated model peak heat flux at the plate is significantly lower than the extrapolated value because neutrals recycled off the plate are allowed to travel very far in the toroidal direction, due to the high neutral assumed toroidal energy of $15 \mathrm{eV}$. The effect of allowing the neutrals to travel too far is that the recycling source becomes less localized, the computed flux amplification factor goes down, and the peak density is reduced as compared to the other simulations because of the smaller particle source near the plate.

\subsubsection{Effect of Momentum Flux (Parallel Viscosity) Free-Streaming-Limit}

All of the analysis done for the Tore Supra tokamak limited momentum flux transport through an effective viscosity term as described in the previous chapter. Because no kinetic predictions of the numerical factor in eqn. (3.15), a value of $I$ was used. A run with the b2 calibrated recycling model was done without a momentum flux limit (i.e. classical parallel viscosity) to examine the effect on the throat plaama conditions.

As shown in fig. 30, the plate plasma density and heat flux without the momentum flux limit are higher in magnitude than the values computed with the momentum flux limit enforced. At fira this difference may appear inexplicable because the ame boundary condition density is appliad along the inner boundary. However the resson for the difference becomes apparent upon examination of the momentum balunce equation (3.2). Neglecting parallel viscosity, radial variations, and tive time derivative, and integrating along a feld line (i.e. assuming that the $x$-direction is the parallel direction), the following equation is obtained:

$$
P(x)=P_{\text {unmom }}-m n N \|^{2}
$$


where all : r ms have been defined in chapter 3 and:

$$
P=\text { lotal (electron plus ion) pressure }
$$

At the entrance to the sheuth, the ion parallel velocity equals the ion sound speed, the second term on the right-hand-side above equals one half of the upstream pressure. Hence the plasma pressure at the plate equals half of the upstream pressure far away froin the plate:

$$
P_{\text {mand }}=P_{\text {unmom }} / 2
$$

This drop in pressure along the field line can occur via a drop in plasma density, temperature, or a combination of both (eq. 3.3). Because the eloctron and ion temperature does not change significantly along a field line for the Tore Supra simulation with active pumping (fig. 20), the plate plasma denaity must drop to about half of the upatream plasma density to satisfy the pressure constraint. The effect of the inclusion of the parallel viscosity term in the momentum equation is that the plasma pressure need not drop all the way to a half of the upstream pressure value. Intuitively, one would atill expect a drop from the upstroam pressure to the downatream pressure because viscosity is a flictional type force which ahould not dominate the momentum transport. However the toroidal protiles (68. 23) show that the pressure does not drop significantly in the toroidal direction when the free-streaming-limit is neglected. The expected pressure drop is present in the case with the free-streaming-limit enforced, which indicates that the magnitude of the clasical (non-limited) viscosity term allows more momentum alux transport than would occur if all of the ions were traveling at the ion sound speed. The eficet on plasma parameters is that a smaller parallel prescure gradient is necesary to satiafy the momentum balance, the plasma density does not drop in the throat (6g. 23), and the plate heat flux increases by a factor of 2 (not shown) because plasma temperature is relatively unafiected by the limit. 
ol Meoma Dendty ve.Toroldel Dietenes from Plate

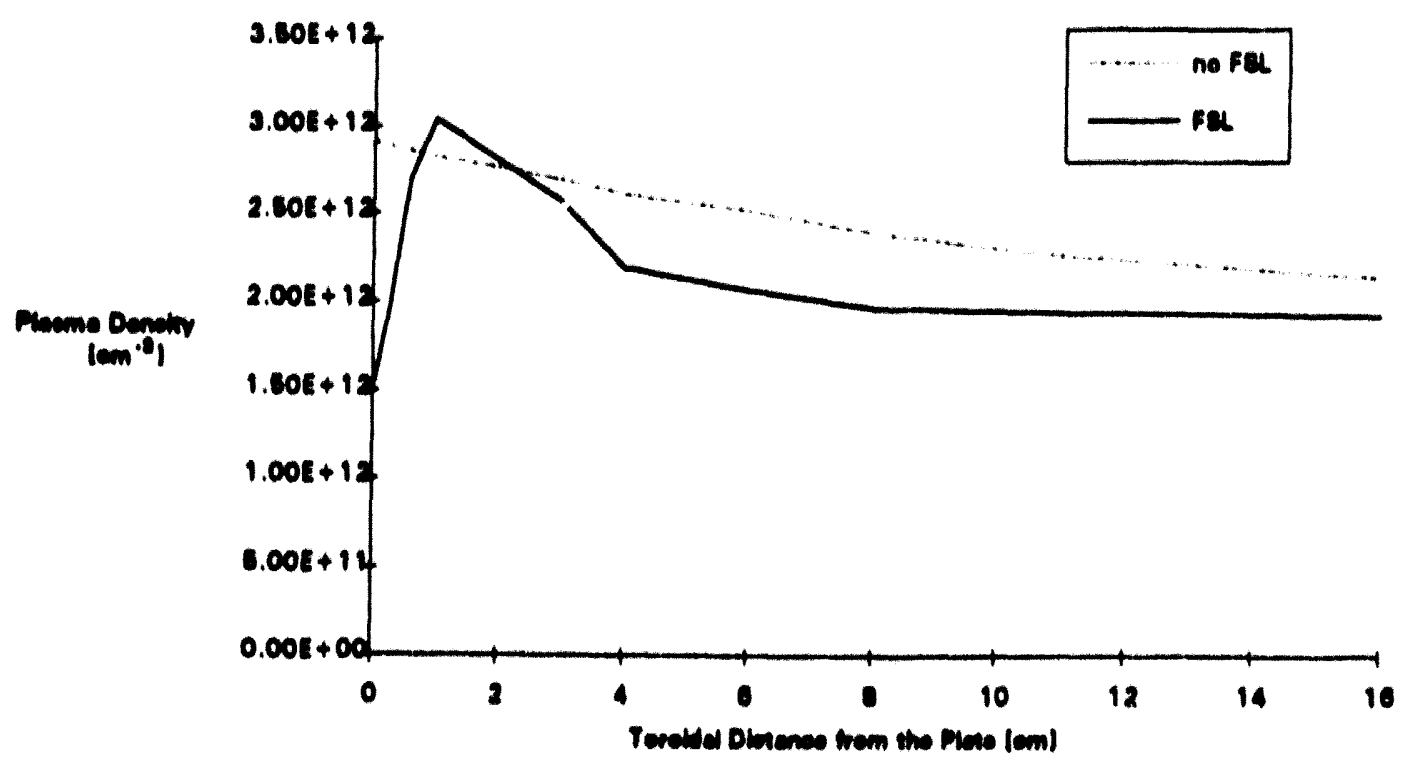

b) Macme Preceure ve.Toroldd Diolenes from Plate

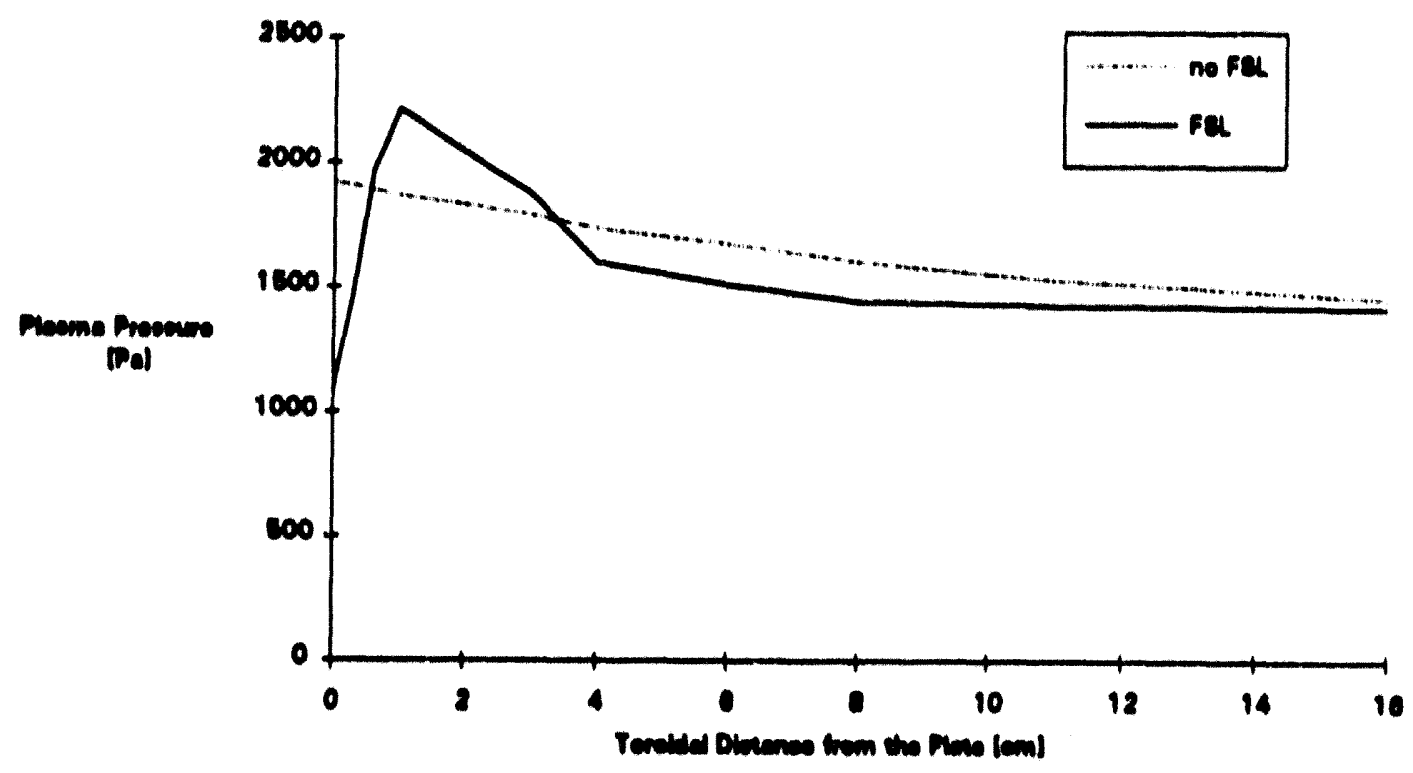

Figure 23: Toroidal prodiles of (a)plasma denaity and (b)pressure in the throat for the calibrated b2 model with and without the panallel viscosity treo-streaming-limit at a radius of $0.4 \mathrm{~cm}$ 
4.2.5. Effect of Pumping on Peak Plate Heat Flux

In an attempt to discover the effect of pumping on peak plate heat flux, the recycling coefficient was varied in the 62 code with the calibrated neutrals model from 0.48 (pumping case) to 0.75 (low pumping speed) to 1.0 (no pumping). All other conditions were maintained to correspond to the benchmark to shot 3127 above. Use of the unity recycling coefficient was intended to simulate shot 3126. A table of relevant quantities is presented below:

Table 12: Variation of Regycline Coefficients for Tore Supre Simulation

Parameter b2/DEGAS

b2

b2

b2

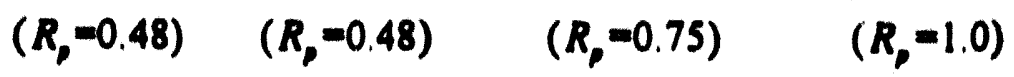

$\Gamma_{1}^{L \infty}\left[\mathrm{cm}^{-2} \mathrm{~s}^{-1}\right]$

$7.6 e 18$

$1.0 \mathrm{e} 19$

$4.4 e 19$

5.9019

$\lambda_{r, \infty}^{r}[\mathrm{~cm}]$

1.7

1.8

$T_{0}^{L}[\mathrm{ov}]$

35

35

30

21

$n_{0}^{L P}\left[\mathrm{~cm}^{-3}\right]$

2.1012

$2.6 e 12$

5.9012

$8.8 e 12$

qis $\left[W / \mathrm{cm}^{-2}\right]$

489

421

490

469

$\int_{\operatorname{lnow}} q_{\operatorname{mon}}[W]$

$6.1 \mathrm{es}$

$4.7 e 5$

$4.6 \mathrm{es}$

4.005

$\int_{i=\infty} r_{1}\left[8^{-1}\right]$

2.0022

2.0022

$2.6 e 22$

3.1022

$\int_{\infty}^{\infty} \Gamma_{1}\left[8^{-1}\right]$

$3.6 e 22$

1.1022

8.1e21

3.1021

- not computed, flow reveral occurred

where

$\Gamma_{1}^{L+}=$ peak particle flux computed at the Langmuir probe location

$\lambda_{r_{1}, p}^{r}=$ radial falloff length of above particle flux

$T_{a}^{L *}=$ computed temperature at location of Langmuir probe 
$n_{a}^{L P}=$ computed density at location of Langmuir probe

$q_{\text {bot }}^{*}=$ computed peak plate heat flux

$\int_{\text {naw }} q_{\text {tod }}=$ total computed plasma energy flowing into the sheath

$\int_{\text {Nate }} \Gamma_{1}=$ total computed plate particle flux

$\int_{\text {cone }} \Gamma_{i}=$ total core particle feed rate into the SOL plasma

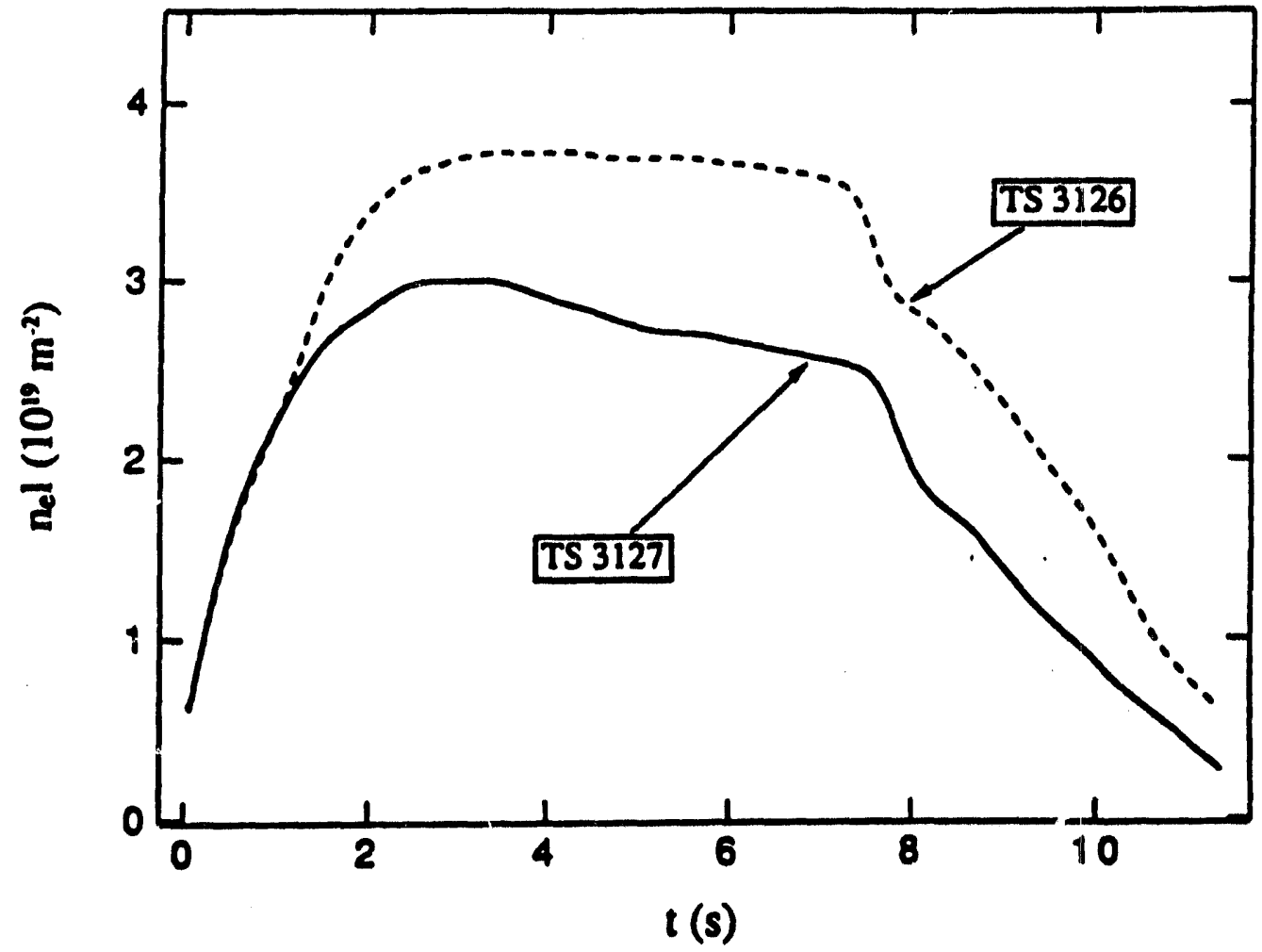

Figure 24: Comparison of line-averaged density for Tore Supra shots 3126 and 3127 
The simulation predicts that the total plasma energy carried into the sheath decreases only slightly when $t$ : ecycling coefficient is increased from 0.48 to 1.0. On the other hand, peak heat flux at the plate is increased slightly, which implies that the increase in density resulting from localized recycling with the higher recycling coefficient dominates the drop in electron temperature under the limiter head. The only significant change that occurs when the recycling coefficient is increased (by changing the pumping speed) is that fewer particles flow from the core into the SOL ( $\int_{\text {con }} r_{1}$ in table 19). The drop in the core outward particle diffusion rate from the unity recycling case to the full pumping case should be large enough to see a significant increase in core line-averaged density. However the lineaveraged density increased by only about $20 \%$ in shot 3126 as compared to shot 3127 (fig. 31). This simulation corroborates the conclusion reached earlier that the particles feeding the SOL must be coming from the wall via outgassing and not from the core plasma. The figure below elicits the magnitude of the plasma density increase at the plate when pumping is switched off:

Pleama Dendity vo. Redlue

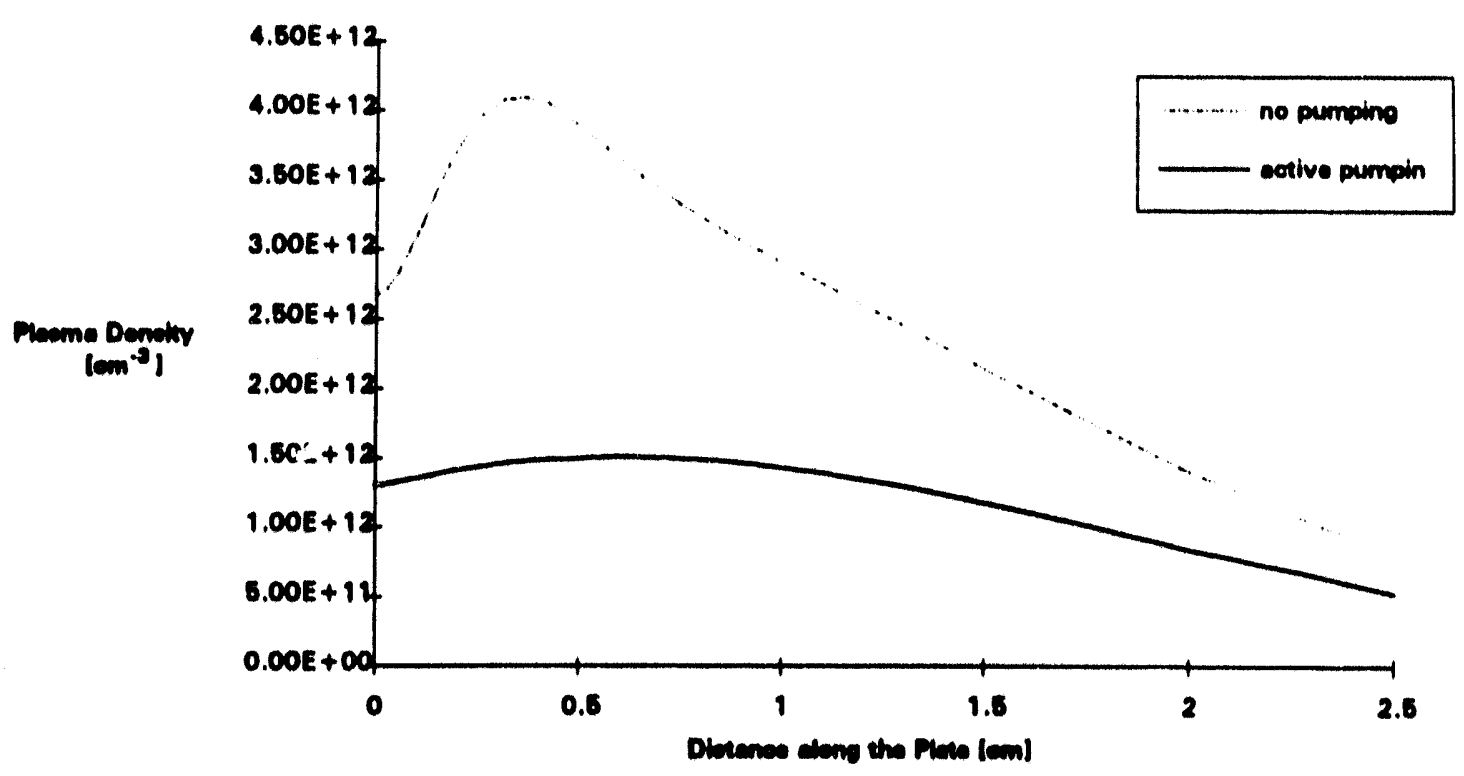

Figure 25: Radial density profiles with and without active pumping 


\section{CHAPTER 5}

\section{CONCLUSIONS}

\subsection{Overview of DIII-D Simulations}

In general, the new molecular physics neutrals model utilized internally in the b2 code computes recycling source terms in good agreement with the coupled b2/DEGAS results. In all but simulations 8 and 9 , the molecular physics model outperforms the singlespecies b2 recycling model in comparisons of the plate peak heat flux and integrated particle flux with coupled b2/DEGAS calculations. Simulation 8 corresponds to the nonorthogonal grid, the results of which need to be confirmed. Simulation 9 neglected the ion conduction free-streaming-limit and does not represent a case of physical interest. Thus the molecular physics model should be used in lieu of the single-species model in all future simulations.

The benchmark of the molecular physics model to experimental data explains features in the measured electron temperature profiles at the plate (double-hump structure) that could not be readily obtained using the b2 single-species neutrals model alone. The presence of the hump in similar shots needs to be investigated. The new model also confirms the importance of the ion conduction free-streaming-limit and allows simplistic simulations of pumping.

\subsubsection{Coupled b2/DEGAS Simulations}

Overall, the coupled b2/DEGAS model has been used to model many different operating scenarios and confinement regimes for the DIII-D tokamak, and has converged in all attempted simulations. The occasional necessity of smoothing the electron energy sink profiles and ion energy charge exchange sink profiles by imposing limits on the 
amount of energy loss in the outer portions of the SOL and the private flux region causes the procedure to require monitoring by the user after each iteration of the b2/DEGAS cycle. Hence the present iterative procedure should not be run in a batch type mode, i.e. several iterations of the b2/DEGAS cycle linked together, and this limitation constitutes one of the two main operational requirements of the coupled code model. The other and more costly requirement of the coupled codes is the large amount of computer time needed for the convergence for a single problem (60 - 200 CPU minutes on a Cray-II). Nevertheless, the coupled codes can and should be used to verify the results of other recycling models. This can most readily be accomplished by using the converged b2 molecular physics neutrals model results as the first guess for coupled b2/DEGAS simulations.

\subsubsection{Molecular Physics Model}

As mentioned previously the molecular physics model almost replicated the source term, plate particle flux, and plate heat flux profiles calculated by the more timeconsuming coupled b2/DEGAS model in all but one simulation. The molecular physics model approximates the b2/DEGAS calculations much better than the original singleneutral-species recycling model in the b2 code.

\subsubsection{Effect of Divertor/Wall Pumping}

The benchmark of the molecular physics model calculations to DIII-D data shows that a plate recycling coefficient of 1 can be used to model both low power and high power input discharges, which reduces the number of variables in the model. The previous benchmarks with the single-species recycling model required a plate recycling coefficient less than unity to match electron temperature and heat flux data. Due to the large particle flux incident on the target, the plate recycling coefficient should approach unity very quickly during a shot. Therefore simulations of steady-state or long-pulse plasma

conditions should use a recycling coefficient of 1.0 in the absence of pumping and less 
than 1.0 when pumping is activated. A more accurate method 10 of simulating wall pumping entails using a plate recycling coefficient of 1.0 and a wall recycling coefficient less than 1.0. It is unclear whether the old b2 recycling model could credibly model divertor pumping because a recycling coefficient less than 1.0 is already needed to model data with no divertor pumping.

Predictions from the molecular physics model indicate that pumping of $3 \%$ of the plate ion flux $\left(R_{p}=0.97\right)$ could increase peak heat flux to the target plates by $10-20 \%$ for the DIII-D tokamak. Athough any increase in peak heat flux is undesirable, it appears that significant density (and impurity) control could be obtained with a relatively small increase in peak heat flux. Therefore, pumping would appear be an effective way of controlling edge and core plasma parameters without unacceptable increases in plate heat flux, provided the particle removal rate is not too large.

\subsubsection{Effect of Ion Conduction Free-Streaming Limit}

As indicated in the analysis chapter, calculations from the 1990 benchmark sequence indicated that more than $40 \%$ of the total heat flowing to the sheath at the divertor plates could be carried by the ion fluid when a free-streaming limit was not utilized in some cases. However when the ion conduction free-streaming-limit is enforced, the ions carry less than $5 \%$ of the total plasma energy into the she ". As expected, the electron temperature increases so that the same amount of plasma ener oy can be carried into the sheath as when the free-streaming limit is not used. Momentum balance requires approximately constant plasma pressure along a field line, which causes the plasma density, and consequently the plate ion flux, to go down. Hence use of the free-streaminglimit drives the SOL plasma into a lower density and higher temperature regime. The main consequence of increasing the electron temperature is that the sheath potential drop increases in magnitude, which increases the kinetic energy of the ions striking the divertor plate and increases the risk of exceeding the physical sputtering threshold limit for the 
divertor material. Other forms of sputtering could also be increased by the increase of the incident ion energy. In view of its importance, the free-streaming-limit should always be used to obtain more accurate estimates of the electron temperature profile at the divertor plates, especially in high power input shots which result in high ion temperatures in the core.

\subsubsection{Use of the Non-orthogonal Grid}

The main feature shown by the non-orthogonal grid calculations by both the b2/DEGAS simulation and the various molecular physics benchmark simulations is that the peak heat flux at the target is not necessarily reduced by the cosine of the incident angle that the poloidal flux tubes make with the floor. In the coupled b2/DEGAS simulation, outboard plate peak heat flux was reduced by less than $5 \%$, much less than the $30 \%$ reduction expected based on geometric considerations. It was indicated earlier in this text that the heat flux might not scale with the cosine of the incident angle because the geometric tilting of the plate with respect to flux surfaces would shift the recycling peak off of the separatrix outer strike point, resulting in a lower electron energy sink and a higher electron temperature at the separatrix, and consequently a higher peak heat flux. However the calculations indicate that the electron temperature peak shifts well off the separatrix at the plate, and that the expected increase in peak heat flux comes from a sharp peaking of plasma density right on the separatrix. The density increase is apparently caused by a strong localization of the recycling particle source at the separatrix, and also by the momentum balance constraint requiring constant pressure along a field line which requires the separatrix density to increase because of the decrease in temperature. In any event, the non-orthogonal geometry of magnetic flux surfaces and the target plates will play a crucial role in determining the heat flux profile along the plate and the particle flux throughput into the pumping chamber. The use of the non-orthogonal grid to model this geometry needs further investigation. In addition an estimate of the non-orthogonal grid 
radial transport inaccuracies needs to be made, and the results need to be examined in greater detail to verify the model credibility.

\subsubsection{Shortcomings and Sensitivities of Individual b2 and DEGAS Models}

The important physics not used in this version of the 62 code for the DIII-D simulations is the effect of grounded (and biased) divertor plates, and electric field fluid drifses. Some of these options are available in the version of the code used to do this analysis, but it was desirable to maintain the same physics used for this and the Tore Supra simulation to provide a general comparison between these two plasma-facing-components. Experimental data from the DIII-D device shows that heat flux on the inboard plate is much lower than predicted by the simulations in the high power input case. This difference may be explained by current flow in the SOL, which flows from the inboard to the outboard plate and carries a large fraction of the inboard side energy to the outboard sides. In addition, radial particle flows caused by electric fields could explain why classical cross-field transport coefficients predict much less transport than observed, necessitating the use of anomalous, large radial transport coefficients.

Selection of the frout parameter in the molecular physics model requires further analysis. It has been suggested 30 that the frowt parameter be scaled with the sine of the angle made by flux surfaces and the physical target plate, even if the modeled target plate is orthogonal to Alux surfaces:

$$
\text { frowt }=0.5 *(1+\sin \alpha)
$$

This scaling should be used as an alternative to constant frowt in future benchmarks for both the DIII-D and the Tore Supra target plates.

Other relevant assumptions in the b2 code discussed in chapter 4 are the values chosen for the energy tranemission factors. The values of these factors are being debated 
on the DIII-D tokamak, and the b2 code calculations are obviously sensitive to these values.

Experimental data are generally not available for relevant atomic physics croussections below $10 \odot \mathrm{V}$, and the values used in the DEGAS code are based on the calculations in Janev33. The reflection coefficient database also suffers from a lack of corroborating experimental data at low energies. The reflection coeficients are important because they directly affect the level of source term localization. Below about 5 eV Incident ion enersy, the backscattered fraction is fixed at $38 \%$, which prevents intense recycling localization. New reflection coefficients for hydrogen and deuterium lons incident on carbon have recently been computed by Ruxic using a fractal model's. These new calculations indicate that the reflection coeficient falls to 0 as the incident ion energy approsches zero so that all cold ions impinging on the divertor plate are desorbed as wall molecules. Use of these new reflection coeficients will undoubtedly have a significant impact on recycling source distributions computed by the DEGAS code (and the b2 molecular physics model).

Finally the effect of the biasing ring and the bafile on the SOL recycling regime has been neglected here. In sctuality, significant recycling sources can occur from plasma interactions with the biasing ring itcalf, especially when the outer strike point is close to the location of the ring. The intensified recyeling sources from plasma contact with the ring will be very important in determining particle tux ariking the plate and oventually difusing into the divertor bafle and pumping chamber. Finally impurity species physics must be added to the models to eatimate the effectiveness of the divertor in confining and removing impurities generated at the target plates and the outer wall.

\subsubsection{Future Work}

The 62 code with the molecular physics model will be benchmarked against date from very high power input (20 MW NBI) shots, as well as the recently discovered VH. 
mode confinement regime. The next stage of the benchmark will match far more diagnostic data than the previous atages: outboard midplane density and electron temperature from the Thomson scattering diagnostic, outboard midplane ion temperature from the charge-exchange-recombination (CER) diagnostic, outboard midplane denaity and electron temperature profiles from a reciprocating Langmuir probe, and neutral pressure messurements under the divertor bafile, in addition to the divertor floor Langmuir probes and RTV measurements.

\subsection{Overview of Tore Supra Simulation}

The simulations of the Tore Supra tokamak were very succesuful in the one regime that was modeled. Unfortunately, very fow masurements were available at the time of the shot, so that the simulation basically matched only two scale lengths. The simulations showed that use of the parallel viscosity free-streaming-limit had a significant impact on the density and heat flux profiles in the pump-limiter throat. However, the simulations also showed that the detail of the 2-D modal calculation may not be necessary to eatimate conditions in the pump-limiter throat when active pumping is used because density and temperature profiles showed little variation in the toroidal direction except very near the plate; a 1-D model may be sufficient for these simulations. The 2-D model is probably necessary for simulations when the pump flaps are closed and pumping is shut off. Recycling within the throat can land to large fux amplification factors without pumping. with a significant impact on plasma parameters in the throat. A great deal of work remains to be done in the 2-D Tore Supra pump-limiter modeling efiort, eapecially comparicon to recent shot mesurements which are more abundant and accurate than previous meseurements.

\subsubsection{Coupled b2/DEGAS and Calibrated b2 Simulations}

The find coupled code b2/DEOAS version has no convergence problems in modeling the SOL regime exiating in shot 3127. More simulations with variation of 
parameters similar to the DIII-D parametric variations need to be done to verify the usefulness of the coupled model, but these simulations have been delayed until a good variance reduction technique is developed in the DEOAS calculation of the pumping chamber to reduce the time requirement, which is 60 Cray-11 CPU minutes per DECAS nun. The b2 portion only requires about I CPU minute per run. Hence the time requirement is the major drawback of using the coupled model, as in the DIII-D simulations.

The callbrated b2 single-species-model appears to be sufficient 10 model the Tore Supra pump-limiter SOL plasme, since the recycling source terma do not dominate the SOL conditions to the extent that they do in the DIII-D divertor plasma. Without active pumping, however, it is posable that the coupling between the plasm and neutrals is atrong enough to require the use of the molecular physics model used in the DIII.D aimulation. A coupled b2/DECAS run using unity recycling is needed to conipare with the results of the calibrated single-species recycling model to draw more detalled conclusions about the effect of pumping on throat plauma parameters.

\subsubsection{Effoct of Parallel Viecosity Freo-Streaming Limit}

Use of the parallel viecosity free-ntreaning-limit was shown to reduce the peak heat flux and plauma dendity at the neutralizer plate by a factor of 2 . The fuct that the computed heat flux matches the measurement-extrapolated heat flux at the leading edge indicates that momentum Aux probably is being phytically limited in the experiment. This particular limit has the strongent effect when the parallel gradient of the parallal velocity is large. For the Tore Supre simulation with setive pumping, this gradient does not vary much in the SOL because the recycling source terms do not affect the flow velocity. When recyeling cource terms are larger, euch es in the Tore Supre experiment with no pumping and all of the DII.D experiments, the fuid now velocity in mont of the SOL is reduced besause many of the reionized noutrals have to dow in the opposite direction (to the other 
drif side of the pump-limiter) to maintain constant pressure along the magnetic field line. Hence the parallel viscosity free-streuning-limit should be used in all pump-limiter aimulations with active pumping, and an eatimate of the appropriate ftactional muliplicative fector, $a^{\prime} m_{0}$, needs 10 be made.

\subsubsection{Pump-Limiter Exhauat Rate}

The variation of recyeling ftom 0.48 to 1.0 indicated that reducing the pumping apeed would increase the peak heat fux at the neutralizer plate slightly but that the total energy carried into the plate would be reduced because of the increased electron impact ionization energy lose due to the increased recycling source localization. Hence an optimization of the pumping epeed could reduce the total enercy carried into the neutralizer plate, albett at the cont of inereasing the peak heas flux 10 the plate and reducing the exhauat rate, which does not have a dratic effect on the measured lineaveraged density anyway.

\subsubsection{2-D ve. I-D SOL Model}

The simulation of shot 3127 showed that density and temperature variations in the toroidal direction were emall except very dose to the plate. Hence the shof could probably have been modeled equally well with a 1.D radial tranapon plauma calculation. The advantages of 1.D models over 2.D models is that they require much less CPU time and 1.D models can include transient behavior calculations. However I-D modals average the 2-D recyeling proalea, wo that regimes of atrong localized recyeling ase not moddad as scourately as 2-D models. Because it is preferable to use the sume modal to analyze pumped and non-pumped shots, the additional time requirement of the 2-D model ( b2 with internal reoyeling) used here does not appear to be a consideration.

\subsubsection{Shortcominges and Senditivities of Individual b2 and DECAS Modds}

The b2 model predietions for the Tore Supre aimulation are very cenative to the aseumed energy tranemiacion actorn at the plate and the boundary conditions used to 
simulate the underaide of the limiter head and the outer wall. The boundary conditions used in this analyais do seem to match the measured date for shot 3127, but it is the only shot analyzed with the model to date, and other boundary conditions may be appropriate for other shots. The ascumed enerey tranamission factors also seem to yield good agreement between computed heat flux at the leading odge and the measurementextrapolated heat flux. Again only one shot has been analyzed.

The other crucial approximation is the geometry of the neutralizer plate, which is aseumed to be perpendicular to the toroidal direction instend of the shallow incidence angle which actually exiass. Tilting the plate in the b2 simulation is expected to increase the radial tranapon of particles and posably reduce paak heat fux 10 the neutralizer plate by shining the density peak radially outward.

\subsubsection{Future Work}

Entimates of the pump-limiter cxhaust rates and the measured line-averaged density drop between non-pumped and pumped shots have led to the conclusion that the particles being exhausted by the pump-limiter are being applied by outgasaing from the outer wall46. The b2 model can be used to dimulate wall outgasaing by simply increasing the wall recycling coeficient above unity and moditying the geometry to be more acourate at the fira wall. The modification entuils increasing the erid radial thickness outside of the throat to the radial location of the phyaleal tira wall.

The moat relevant improvement to the Tore Supra tokamak model pertains to the modeling of the entire SOL and not juas the throst, as mentioned in the description of the stairutep modd. This uperade of the 2-D geometry will allow calculation of the total enercy incident on the pump-limiter as well as extimates of all of the plasma conditions at the LCFS. An alternative to the rairatep approximation of all thed aurbees is the nonorthogonal geld technique ued for the DIII-D dimulations. Both techniques will be teated as methods to mudel tilted targets on the Tore Supra tokemak. 
None of the simulations done for Tore Supra enforced a free-streaming-limit on ion conduction parallel to field lines. In view of the conclusions from the DIII-D analysis, additional Tore Supra simulations using the flux limit should be done to verify the present results. Fortunately, the present resules will probably change only slightly since low temperature conditions existed along the modeled inner boundary and in the pump-limiter throat.

Finally, the b2/DEGAS coupled model needs to be applied for many different regimes, $\boldsymbol{w}$ in the DIII-D simulation, and the results then used to calibrate the singlespecies or molecular physics recyeling model. Then the calibrated model should be used to analyze recent shots which have IRTV measurements of heat flux along the plasma-facingsurface of the limiter and more precise Lengmuir probe measurements.

\subsection{General Commenes}

\subsubsection{Divertor vs. Pump-Limiter SOL}

One of the original goals of this study was to provide a gross comparison of the effectiveneas of pump-limiters and divertors as plasma-facing-components. Early in the study, however, the fact that pump-limiters and divertors are two completely different devices, with similar but not identical tacks, became apparent. In general, pump-limiters muat be able to withatand much larger heat loads than divertors because of their proximity to the hot core plasma. On the other hand, pump-limiters are considerably less expensive than divertors and appear to be auficiently eficetive in particle exhaust. Heat flux probably being the limiting fetor, a single pump-limiter could not survive in the high heat loads envisioned in operation of power-producing reacton. Because pump-limiters are oriented at a very shallow angle to Geld lines, magnetic fold ripple near the limiter could simificantly inerease the peak heat dux to the limitert? Of course, one of the attractive features of pump-limiten is their modularity, enabling the use of soveral modules within a given tokamak. Multiple pump-limiten can be used in share the energy diffusing into the 
SOL from the core, but the other problem accompanying proximity to the core is physical and chemical sputtering of the limiter because of the high electron and ion temperatures close to the limiter.

\subsubsection{One General Code for Plasma-facing-component Analysis}

The objective of analyzing a pump-limiter SOL and a divertor SOL with the same technique/set of codes has been accomplished, but the calculations show that different phyaics dominates pump-limiters and divertors. In order to fulfill the above objective, relevant physics was neglected in the DIII-D divertor simulations. Hindsight indicates that the physics pertaining to fluid drifts and grounded neutralizer plates should have been used for both simulations, which would have entailed a full toroidal simulation of the pumplimiter from the electron drift side to the ion drift side. This simulation may have answered questions about toroidal asymmetries 48 of plasma parameters in pump-limiters. Nonetheless, the success of the coupled b2/DEGAS model, the molecular physics mociel, and the calibrated single-species recycling model represents a significant advance in SOL modeling by taking into account the coupling between plasma and neutrals in a detailod manner. 
(through the process of quantum mechanical tunneling) and collide, staying together sufficiently long to fuse together. High random thermal energy by itself is not sufficient to ensure production of significant amounts of fusion energy. The ions and their energy must also be contained by the confining magnetic field long enough to ensure a high core plasma density and probability of fusion events. One measure of how long ions stay within the core is given by the particle confinement time, $\tau_{p}$. The corresponding quantity for energy is called the energy confinement time, $\tau_{E}$. Maximizing $\tau_{p}$ for fuel ions and $\tau_{E}$ helps to increase the fusion power production rate.

In the early 1980's, an operating regime during which both $\tau_{p}$ and $\tau_{E}$ were enhanced was observed on the ASDEX tokamak ${ }^{49}$. The magnetic configuration in which the enhancement was achieved was a divertor (see the section on divertors in this appendix for more information). This confinement regime was termed $\mathrm{H}$-mode (high confinement), and it occurred with the usage of large amounts of neutral beam (NBI) power. The other confinement regime in which smaller amounts of NBI power were used and which resulted in lower values of $\tau_{p}$ and $\tau_{\varepsilon}$ was termed L-mode (low confinement). H-mode is characterized by reduced divertor (or limiter) heat flux to the plasma-facingcomponents, an increase in $\tau_{p}$ and $\tau_{B}$ over L-mode, steepening of the radial edge plasma density and temperature profiles, and the presence of disruptions called edge-localizedmodes (ELMs). It has since been discovered that specific plasma conditions in the SOL accompany $\mathrm{H}$-mode, such as reduced impurity neutral penetration into the core plasma from the SOL. Recently a new confinement mode (VH-mode) resulting from a boronization process of the first wall has been discovered on the DII-D tokamak ${ }^{37}$. Impurity influx to the core from the SOL plasma is reduced and the core confinement times are larger than $\mathrm{H}$-mode values. It is unclear whether the enhancement of the core confinement parameters causes the changes in the SOL plasma conditions or is caused by those same changes. Nonetheless accurate modeling of the SOL plasma is necessary 
because the core confinement regime appears to be strongly coupled to the SOL plasma conditions.

\subsection{Boundary Layers}

\subsubsection{Definition and Basic Physics Description}

The plasma BL can be described ${ }^{35}$ as two separate regions: the radiating layer $(R L)$ and the SOL. The SOL extends between the outermost magnetic flux surface which does not strike a material boundary, the last-closed-flux surface (LCFS), and the vessel wall (fig. 26). The thickness of the $R L$ depends on the specific types of impurities and concentrations present; for common light impurities like oxygen and carbon, the $R L$ generally extends a few centimeters radially inward from the LCFS ${ }^{35}$. The tokamak SOL plasma has fluid-like properties, even in collisionality regimes where kinetic effects are expected to dominate, whereas the $R \mathbf{L}$ plasma is influenced by atomic physics processes. The physical distinction between the two layers is not always clear, because significant amounts of radiation can also occur in the SOL.

The motion of hydrogenic and impurity neutral atoms and molecules bom from plasma-wall interactions enter the core plasma unirhibited by the magnetic field. Hence cold neutrals move away from the walls inward toward the hot plasma and cause energy losses to the plasma ions via charge exchange (CX) and the plasma electrons tia electron impact ionization (EII) and inelastic processes. A dense SOL plasma would maximize EII efficiency of the neutrals in the boundary layer, preventing the neutrals from entering the core plasma and localizing EII losses to the SOL. Localizing EII sinks in the SOL would cause SOL electron temperature $\left(T_{e}\right)$ to fall, which in turn would reduce wall erosion. The concept of creating a cold, dense plasma SOL to shield the vessel wall from the hot core plasma is a very active area of edge plasma physics.

\subsubsection{Parallel and Perpendicular Transport in the Scrape-off-layer}

In a tokamak, particle and energy transport parallel to the magnetic field dominates perpendicular (radial) transport. Parallel transport is especially dominant on magnetic field 
lines that intercept material boundaries or targets. Since the parallel electron thermal speed is much greater than the ion speed, a negative electrostatic sheath forms in front of the target to equate electron and ion flow to the target. The potential drop across the sheath is on the order of several times the local electron temperature. In order to maintain the sheath, the Bohm criterion legislates that the ion fluid flow velocity must equal the ion sound speed, $C_{\imath}$, at the sheath entrance where $C_{d}$ is given by 50 :

$$
C_{1}=\sqrt{\frac{k_{b}\left(T_{1}+T_{1}\right)}{m_{i}}}
$$

where

$$
\begin{aligned}
& T_{b} T_{i}=\text { electron and ion temperatures } \\
& m_{i}=\text { ion mass } \\
& k_{b}=\text { Boltzmann's constant }
\end{aligned}
$$

$C_{\text {, }}$ is usually greater than $10^{5} \mathrm{~m} / \mathrm{s}$ for most tokamak operating SOL conditions. By contrast radial diffusive drift velocities are usually less than $100 \mathrm{~m} / \mathrm{s}$. Hence both particles and heat can be efficiently channeled to a target via parallel transport instead of the first wall, which receives particles and heat via cross-field transport. The Bohm condition is also important because it is a boundary condition for the momentum balance, pinning the ion fluid velocity at the entrance to the sheath boundary near the plate. The acceleration of the ions to the sound speed increases both the particles and energy transmitted to the target plates.

\subsubsection{Scrape-off-layer Engineering}

As stated previously, the Bohm criteria prescribes that the ion fluid velocity equals the ion sound speed at the sheath entrance. The ion particle flux, $\Gamma_{1}$, is given by $n_{1} v_{1}$, and at the plate $\Gamma_{i}=n_{i} C_{s}$. Hence the plate ion flux is proportional to $n_{1} T_{1}^{0.5}$ or $n_{p 1} T_{p}^{0.5}$ if $n_{1}=n_{e}=n_{p}$ and $T_{i}=T_{i}=T_{m}$. Heat flux at the target is related to particle flux and temperature at the target by:

$$
q_{i o t}^{N \infty} \propto \Gamma_{i} * k_{b} T_{i}+\Gamma_{0} * k_{b} T_{0} \propto n_{N} T_{N}^{3 / 2}
$$


Momentum balance along : field line constrains the parallel pressure $\left(2 n_{p l} T_{p l}\right)$ to be approximately constant aw: irom the plate. At the plate, the plasma pressure drops to about half of the upstream pressure (due to the kinetic energy of the ions). The previous relationships indicate that reduction of heat flux to the target would occur if the plasma temperature were somehow lowered (provided the plasma density rose more slowly than $T_{p 1}^{3 / 2}$ ). The next subsections on PFC will explain how PFC are used to lower $\tau_{p}$ in the SOL. The pressure balance would then cause the plate particle flux to increase. Increasing the plate ion flux will further aid in the transition to the desired cold, dense SOL.

The SOL ionization efficiency must be very high to localize recycling sources within the SOL. The dependence of the collisional radiative electron impact ionization $<\sigma \nu\rangle_{\text {al }}$ parameter on $T_{\text {a }}$ can be approximated ${ }^{l}$ as follows:

$$
\langle\sigma v\rangle_{m}=3 \times 10^{6} * \frac{T_{e}^{2}[\mathrm{eV}]}{3+0.01^{*} T_{e}^{2}}\left[\mathrm{~cm}^{-3} \mathrm{~s}^{-1}\right]
$$

The $\left\langle\sigma \nu>\sigma u\right.$ parameter falls off sharply for $T_{\text {, less than }} 10 \mathrm{eV}$, but saturates quickly for $T_{\text {c }}$ greater than $10 \mathrm{eV}$ (fig. 27). The local SOL ionization reaction rate is given by :

$$
S_{1}=n_{0} * n_{n} *<\sigma \nu>\sigma
$$

where $n_{n}$ is the neutral density. Near the targets, the ionization rate peaks and is related to the local plasma density by:

$$
\left.S_{i} \propto n_{0} R_{p} n_{i}<\sigma v>_{m i} \propto n_{N}{ }^{2}<\sigma v\right\rangle_{m}
$$

Recalling that the momentum balance constrains plasma pressure $\left(2 n_{N} T_{N}\right)$ to be constant along field lines and using core/SOL interface conditions of $T_{\mu}=100 \mathrm{eV}$ and $n_{\mu}=2 * 10^{19} \mathrm{~m}^{*}$ 3, $S_{i}$ peaks for $T_{\mu}$ between $10 \mathrm{eV}$ and $20 \mathrm{eV}$, which corresponds to $n_{\mu}$ between $10^{20} \mathrm{~m}^{-3}$ and $2 * 10^{20} \mathrm{~m}^{-3}$. Hence a cold (but not too cold), dense SOL relative to the hot core plasma indeed maximizes the SOL ionization rate of neutrals born at the target plates from recycling. 

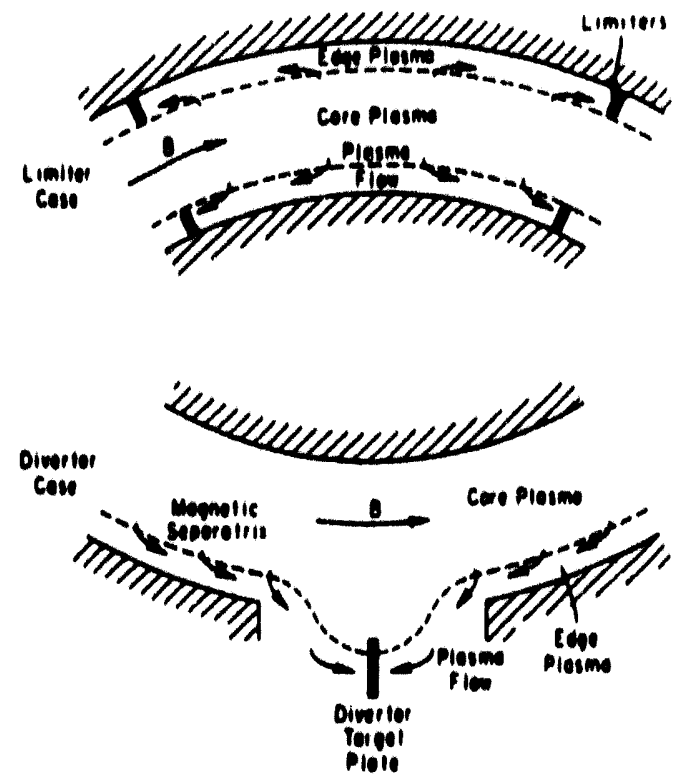

Figure 26: Schematic of core plasma and edge plasma boundary and the last-closed-fluxsurface (LCFS) of a typical limiter and divertor tokamak29. The LCFS is indicated by the dashed lines.

Ebetren unpect lonization Rosetion Rate ve. Ebetron Temperature

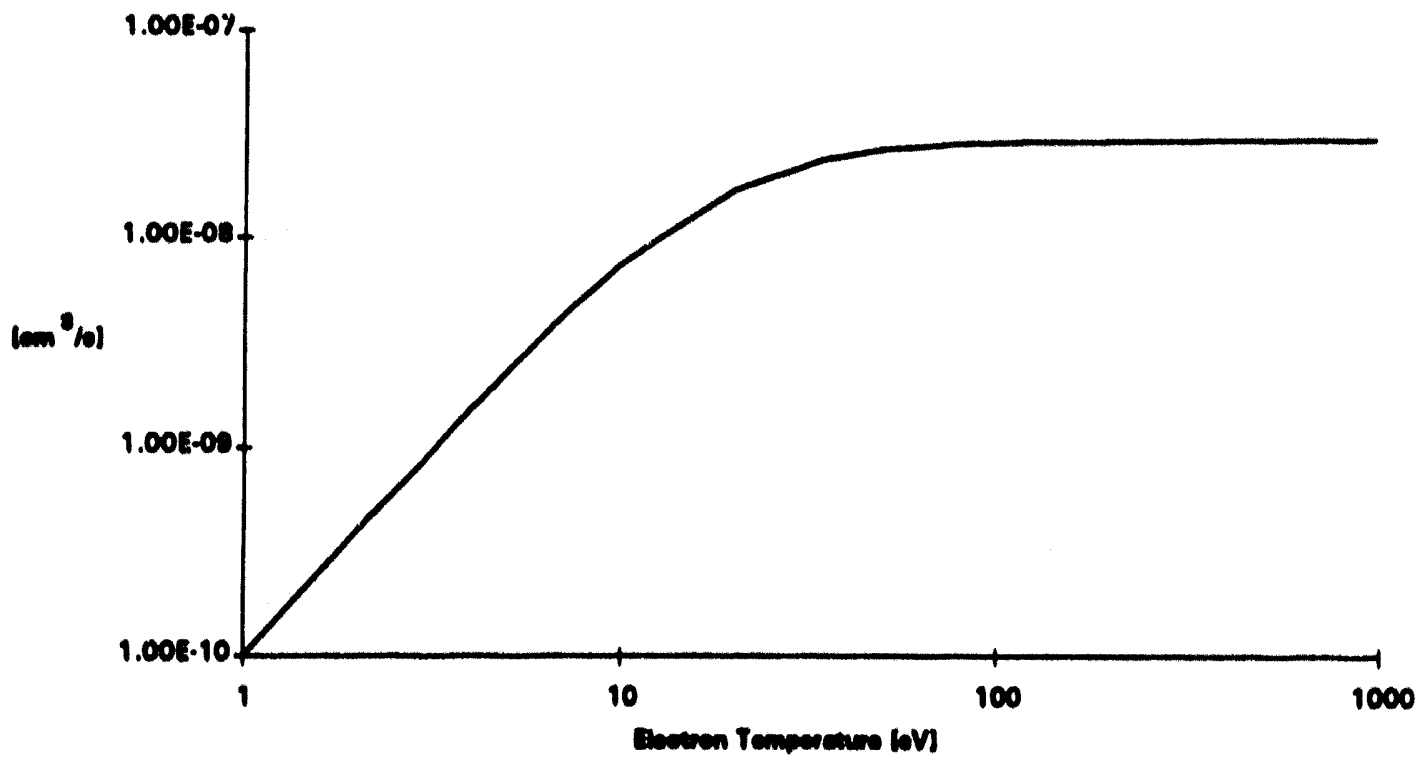

Figure 27: Dependence of the electron impact ionization reaction rate coefficient (hydrogen) on the electron temperature (approximated) 1 


\subsection{Plasma-facing-components Configurations}

One of the first methods to utilize the dominance of parallel transport over crossfield transport to channel heat and particles through a SOL to a target was installation of a limiter, a sacrificial surface jutting radially in from the vessel wall (fig. 28). This limiter is the primary site of plasma-wall interactions and can be replaced periodically. The surface(s) on which the ion flux impacts is called the neutralizer or target plate(s). Use of a limiter can extend first wall lifetimes by many years. Limiters can be poloidally symmetric (poloidal limiters), axi-symmetric (toroidal or rail limiters), or non-symmetric in both the poloidal and toroidal planes (button or mushroom limiters). Pump-limiters are limiters with pumps attached to aid in particle and impurity control (fig. 29). In addition, multiple limiters can be used in the same tokamak to spread the plasma heat and particle load over several modules.

Scaling of present limiter tokamak data indicates that a plasma-facing-component (PFC) system consisting solely of limiters may necessitate replacement of the system too frequently in fusion reactor operation. Because the limiter SOL has low plasma density, shielding of the core plasma and the first wall from each other can be sufficient, but shielding of the limiter from the core plasma may not be sufficient to allow optimal core performance and minimal limiter damage.

One method to create a cold, dense SOL is the magnetic divertor. The magnetic divertor diverts a field line of the minority magnetic field in the SOL away from the core plasma, sets up a magnetic null point (the x-point), and diverts the SOL plasma toward a set of target plates. Diverting the magnetic field requires an extra set of magnets to partially offset the existing field and neutralize it completely at the x-point. The minority field is diverted to minimize the effect on core confinement; in tokamaks, the poloidal magnetic field is generally diverted and the magnetic configuration is referred to as a poloidal-field divertor (fig. 30). Poloidal-field divertors can have any number of magnetic nulls in a given poloidal cross-section. Operation with one null is termed single-null (SN) 
1. "eimple" wall contect

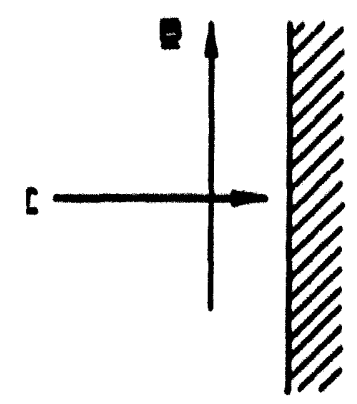

2. wall eentaet eoneentrated at llmiter

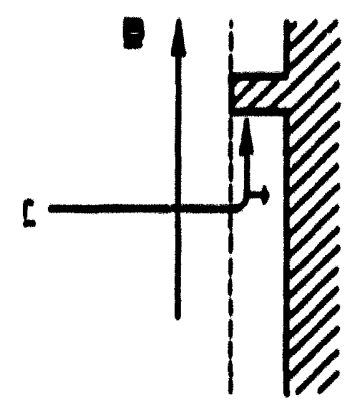

3. wall eontaet eoneentrated in divertor

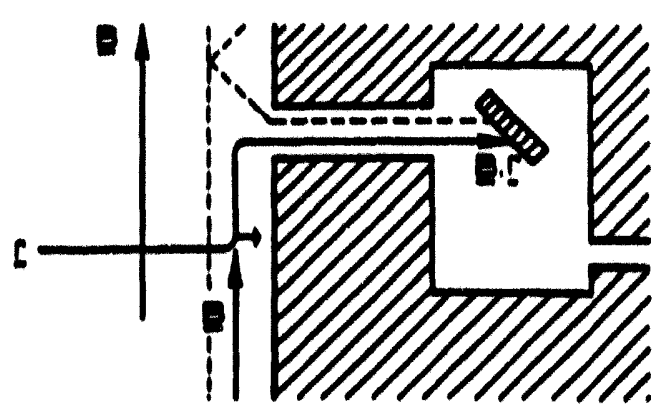

Figure 28: Illuatration of the various types of plasma-wall contact, taken from Post51. 
and operation with two nulls is termed double-null (DN) (fig. 31). DN configurations provide two targets over which to spread heat and particle fluxes but shorter field line connection lengths for radial flux spreading. Nevertheless, the DN configuration may offor the ben operating ecenario for reactors. Rescarch on moat tokamak divertor experiments is done usually in the SN configuration, however, due to the higher cont of operation in the DN configuration. The divertor configuration has the advantage of physically separating the location of plasma-wall interactions and the core plasma, which prevents neutral impurity particles from directly entering the main plasma. A large frection of thermal energy can often be dissipated by plasma-neutral interactions (radiation) prior to contact with the target plates. Closed divertors (i.e. ASDEX, fig. 31) include a separate chamber where plama/wall interactions take place, and open diventors (fig. 30). allow contact in the main chamber. As with limiters, pumps can be used in conjunction with divertors to optimize particle control in the boundary layer. The DII-D device will be the firat large tokamak to inatall a pump in conjunction with the divertor in September, 1992.

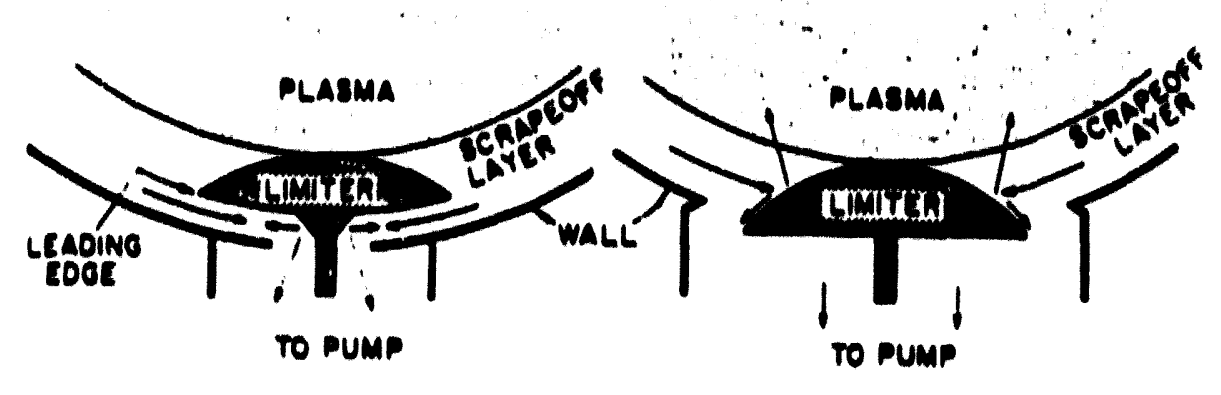

(.) GLOSEO CONFIOURATION

(D) OPEN CONFIOURATION

Figure 29: Schematic of open and cloced pump-limiter conffourations, uken from Mioduczewakjs2. 


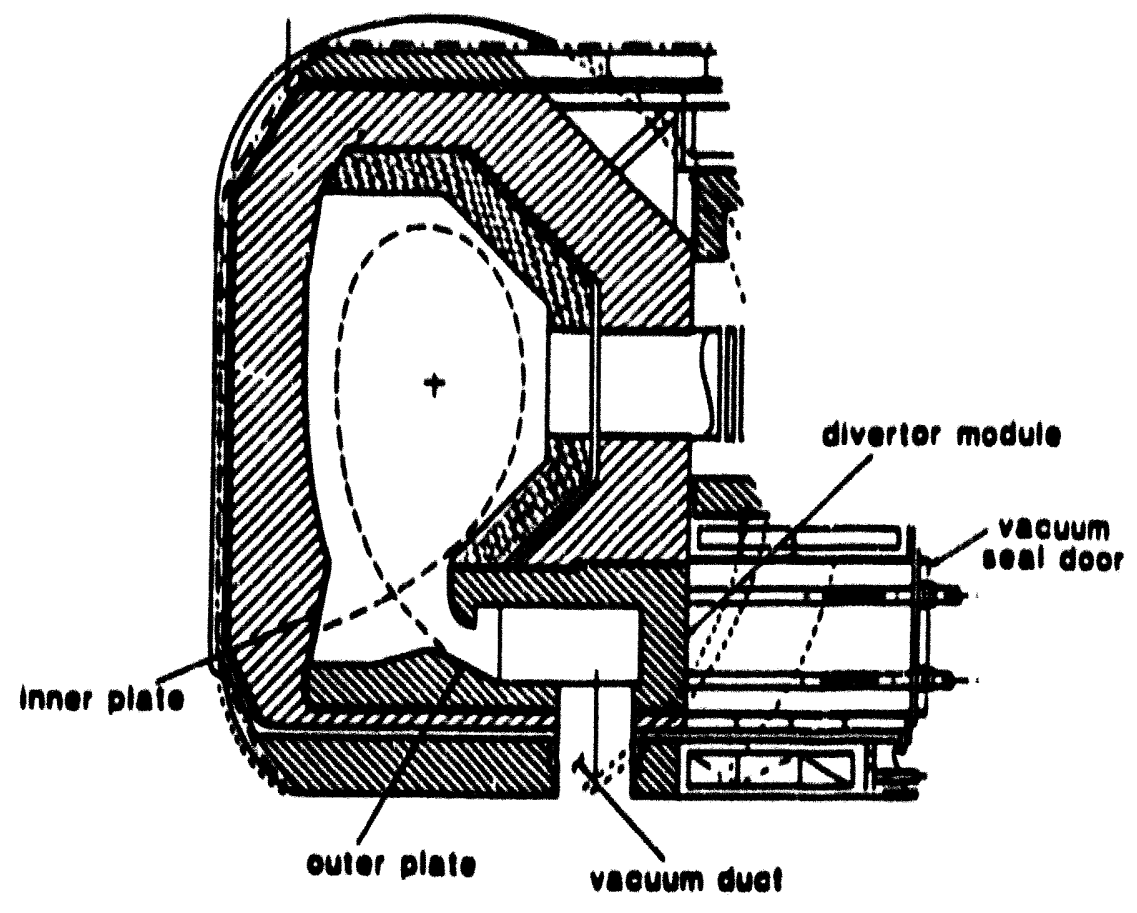

Figure 30: Schematic of the conceptual DNTOR poloidal divertor, uken from Ponts!.

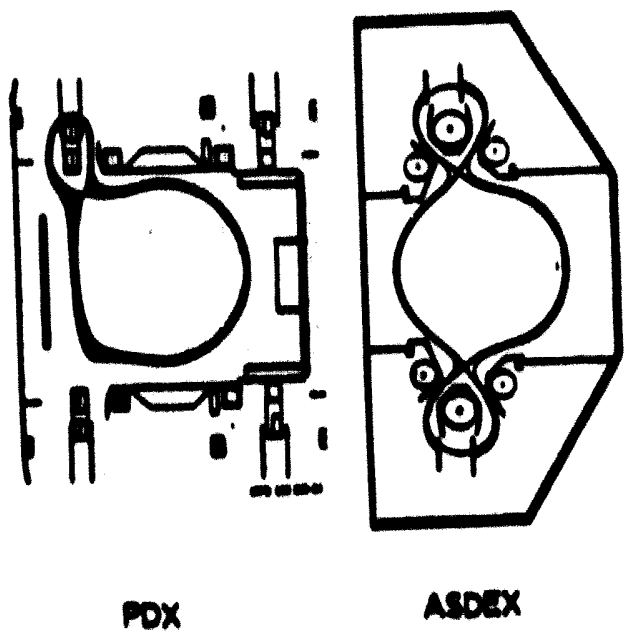

Figure 31: Diagram of a ainglo-null divertor (PDX) and a double-null divertor (ASDEX), tuken trom Wamer a Lackners3. 


\section{APPENDIX B}

\section{Examples of O.D and 1.D SOL Plasma Models}

Post offers an excellent eurveys4 of pre-1986 SOL plasma models and much of the discusalon of historical plasma model development below follows that review. The discusation of O-D and 1-D models is not intended to be a comprehenaive survey; the specific examples discussed are representatives ffom the literature which are relovant to this work.

\subsection{Zero-Dimensional Plauma Models}

The simpleat plauma model is obeained by replacing the parallel and perpendicular transpon terms in the conservation equations by global characteriatic loss times for particles, momentum, and enerey:

$$
\frac{\partial A_{1}}{\partial t}-s_{1}-\frac{d_{1}}{r_{1}}-\frac{A_{1}}{r_{1}}
$$

where

$1=1,2,3$

$A_{1}=n_{N}$ (plasma dendity)

$A_{2}=m_{1} n_{N^{v}}$ (plasma momentum)

$A_{3}=3 / 2 n_{N}\left(T_{0}+T_{1}\right)$ (plasma internal enorgy)

$S_{1}$ - the sum of all cources and sinks for a lluid property

$\eta$ - characteriatic parallel loss time

$i_{1}$ = characteristic perpendicular loss time

These O-D models have been used for aimulations of lons sources used to make neutral beams and for plauma atartup transient analycisst. The obvious deficiency in these models is that information on plasma parameter variation in any direction is not available. 


\subsection{One-Dimenulonal Plasma Models}

One-dimensional plasma models offer a wide range of complexity. The simplest 1. D modals examine radial transpon with a loss lerm for the parallel transpon. Plasma parameters are seaumed to be conotant along field lines, which is generally a good asumption for a limiter SOL or a low recyeling divertor SOL. In the high density, low temperature regime characteriatic of high recyeling divertor operation, I.D model considering parallel transport and neglecting radial transpon is a Mir approximution. Examples of a parallel tranepon modal and a perpendicular transpon model are described below and representative calculations from the literature are also presented.

\subsubsection{One-Dimensional Radial Trunsporn Models}

Considering the ateady-atate particle and enercy balances and neglecting the momentum balance, the transpon cquations take the form :

$$
\begin{aligned}
& \frac{\partial}{\partial x}\left(D_{1} \frac{\partial n}{\partial x}\right)-\frac{n}{\eta}+S_{n}=0 \\
& \frac{\partial}{\partial x}\left(n x_{1} \frac{\partial T_{1}}{\partial x}+\frac{3}{2} T_{1} D_{1} \frac{\partial n}{\partial x}\right)-\frac{2 r_{1} n T_{1}}{\eta}-Q_{1}+S_{t}=0 \\
& \frac{\partial}{\partial x}\left(n x_{1} \frac{\partial T_{1}}{\partial x}+\frac{3}{2} T_{1} D_{+} \frac{\partial n}{\partial x}\right)=\frac{2 n r_{1}}{9}+Q_{L}+S_{L}=0
\end{aligned}
$$

where $x$ is the coordinate normal to magnotic aux eurfeces, i.e. the radial coordinate, and where:

$D_{1}=$ radial diaruion coetweient

$x_{1} \cdot x_{1}$ - radial alootron and ion thermal difusivity

$n$ - plaema denalty

$y_{0}$ - dectron enerogy tranamiedion coefselent

$Q_{\Delta}$ - claseical equipartition enerey transfor term between olectrons and ions

$s_{f}, s_{p}=$ recyeling enercy cource for elcotrons and ions 
All other times have been defined. Ulickson used a model with these foatures and compared results of the model to Langmuir probe data from PLT and PDX. By comparing many nuns, he was able to obtain the dependence of the difinuion coefficient, $D_{1}$, and the elcetron thermal diffusivity, $x_{1}$, on plasma denalty and temperaturest:

$$
\begin{aligned}
& D_{1}=(1 \pm 0.3) m^{(01023)} T^{(0102)} m^{2} / s \\
& X_{1}=(\theta \pm 3) \cdot 10^{12} n^{(-11003)} q(0.023) m^{2} / s
\end{aligned}
$$

The above scaling is relovant because it found no definitive dependence of the anomalous radial transpon coefitionts on the plasma temperature, which corroborates the use of constant diffusivities in the 2-D simulations of the Tore Supra pump-limiter and the DIuD divertor. However the scalling also indicates that the radial dectron thermal diffusivity ahould be sealed with plaema denaty. Unfortunately, it is not known whether the scaling indicated above is applicablo in Wigh reoyeling regimes, and the aforementioned model's appliability may be reatrieted to a low denaity SOL.

\subsubsection{One-Dimenalonal Paraldal Transpon Models}

In high recycling divertors, there can be signiffoant variation of plasma parameters along field lines, which invalidates the sexumptions of the above 1.D model of aseuming plaema parameters to be conatant along fidd lines. One example of a 1-D parallal tranapon model is the one used by Mahdavi for almulation of high recycling divertor experiments on the DII-D tokamakss. In this model crose-field transpont and particle transpon (except near the target) are negleoted. The plaema channol is depicted as a rectangular channel of lenget / and croserection $A_{x}$ with a power cource, $P_{0}, a x=0$ and the wall at $x=1$. The denaity of elcetrons af $x=0$ is aseumed to be proportional to the average plama denaty, $\bar{n}_{0}$. In this coenario, the electron enerey equation retains only the dominant conduction term. The continuity and conduction cquations are liated below: 


$$
\begin{aligned}
& \nabla_{1} \cdot x_{1} \nabla_{1} k T_{c}=0 \\
& \nabla_{1} n_{c} T_{c}=0 \\
& x_{1}\left(T_{c}\right)=x_{0} T_{c}^{2 n}
\end{aligned}
$$

where $x_{0}$ is a constant. Integrating the conduction equation and applying boundary conditions leads to the following solution for electron temperature in extremely high density regimes:

$$
\begin{aligned}
& T_{0}(x)^{n n}=T_{c}(0)^{n}-7 P_{0} x / 2 x_{0} A_{d} \\
& T_{0}(0)=\left(7 P_{0} / / 2 x_{0} A_{1}\right)^{2 n}
\end{aligned}
$$

At the target plates the cealing of divertor parameters with bulk plasma density, $\bar{n}_{0}$, is given by:

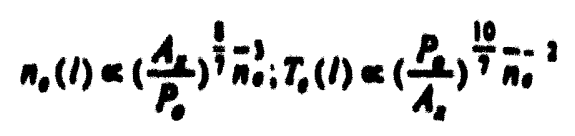

This type of ecaling was indeed observed on the DIII-D tokamak in high recyeling regimesss, although the magnitudes of the predicted pressures do not exectly match the data. This model confirms that electron conduction is indeed the dominant term in the energy balance and muat be computed accurately for a valid simulation. Freo-streaming limits on electron and ion conduction play a crucial role in predictions of heat flux and are diceussed in the main body of the text. 


\section{APPENDIX C \\ Non-orthogonal Grid Generation Procedure}

The orthogonal grid-generation procedure ${ }^{5}$ is based on EFITD ${ }^{38}$ fits to magnetic probe measurements for particular shots and is described only briefly. The basic procedure entails a transformation of a uniform rectangular grid of magnetic flux $(\Psi)$ values to a computational mesh of $\mathbf{R}$ (major radius) and $\mathbf{Z}$ (height) values with grid points lying parallel and perpendicular to surfaces of constant magnetic flux. Firat a biquadratic fit is applied to magnetic equilibria to generate the constant magnetic flux surfaces on the grid, and all saddle points are identified. Next the grid is corrected to portray the effect of target plates, the firat wall, and any other material surfaces in contact with the plauma. The grid physical diatance from the x-point to the plate is generally selected to match the experimentally measured value for a particular shot. Because the physical divertor plates (the vessel floor) are not necessarily orthogonal with respect to magnetic lux surfaces, the grid usually extends below the vessel floor in the private-flux-region area and terminates above the vessel floor in the outer ecrape-off-layer. The non-orthogonal grid-generation algorithm for lower single-null divertor configurations is described below. This algorithm has not yet been generalized to allow construction of meshes based on double-null divertor configurations.

First, the orthogonal grid is generated with the length of the outermost radial Aux tube (in the outer part of the SOL) selected to intersect with the divertor floor. Consequently, all other flux tubes extend below the vessel Aloor. Next, the distribution of meah points slong all of the flux tubes which extend below the floor is recomputed. This redistrihution is only used for mesh points which lie below the x-point; all mesh points 
above the x-point are not affected. For example, consider the row of grid points along the separatrix. The arc length from the $x$-point to the $n^{\text {th }}$ mesh point $\left(d x_{n}\right)$ along the separatrix is computed, as is the arc length from the x-point to the last mesh point (dl) on the separatrix. Next, the arc length from the x-point to the physical vessel floor (df) is computed. The $\mathrm{R}$ and $\mathrm{Z}$ location of each mesh point below the $\mathrm{x}$-point along the separatrix is then recomputed based on the ratio: $d x_{n}^{*} d f / d l$. The actual grid point redistribution algorithm insures that the recomputed $R$ and $Z$ values for mesh points lie along the separatrix. The procedure is repeated for all of the grid cells lying along the other flux surfaces. For those flux surfaces, the arc length $\mathrm{dx}_{\mathrm{a}}$ is computed as the distance from the mesh point which has the same poloidal index as the $x$-point to the $n^{\text {th }}$ cell. This procedure insures that the 2-D grid is tied to magnetic flux surfaces in the poloidal direction, which insures that the plasma parallel transport is computed properly. However, the radial distribution of mesh cells is not necessarily orthogonal to poloidal flux surfaces.

The non-orthogonal grid-generation algorithm described above insures that large changes in cell volume do not occur in adjoining cells. Non-orthogonal cells may exist in the grid up to the $x$-point. However the radial distribution of cells is almost normal to poloidal flux surfaces at the x-point and becomes only appreciably non-orthogonal very near the plate. The non-orthogonality in the grid causes a error in the calculation of radial transport (see chapter 4, simulation 8), the magnitude of which is not addressed in this study. 


\section{APPENDIX D}

Detailed Comparisons between the Two-Species Model and b2/DEGAS Codes

Detailed comparisons between the b2/DEGAS model and the two-species model for the nine parameter variations presented in the DIII-D analysis section are tabulated here. The enhanced radiation molecular physics model entails a $10 \%$ increase of all inboard electron energy sources to better estimate inboard peak heat flux. All of the terms listed in the following tables are defined below, and the units are given in the square brackets:

$S_{n}^{\mu k}$ (in, out) [ $\left.\mathrm{cm}^{-3}\right]$ - peak particle source terms on inboard and outboard sides $\lambda_{\mathrm{s}}^{\text {rad }}$ (in, out) [cm] - radial falloff length of peak particle source terms $\lambda_{s_{n}}^{\text {pld }}$ in, out) [cm] - poloidal falloff length of peak particle source terms $S_{\infty}^{\mu k}$ (in, out) $\left[W / \mathrm{cm}^{3}\right]$ - magnitude of peak electron energy recycling source terms $\lambda_{s_{\alpha}}$ (in, out) [cm] - radial falloff length of peak electron energy source terms $\lambda_{s_{\infty}}^{m^{2 l}}$ (in, out) [cm] - poloidal falloff length of peak electron energy source terms $\int S_{n}$ (in, out) $\left[8^{-1}\right]$ - integrated particle source term for each plate $\int S_{\infty}$ (in, out) $[W]$ - integrated electron energy source terms $\int S_{\alpha}$ (in, out) $[\mathrm{W}]$ - integrated ion energy source terms $n_{a}^{\text {evp }}$ (in, out) $\left[\mathrm{cm}^{-3}\right]$ - separatrix density at the inboard and outboard plates

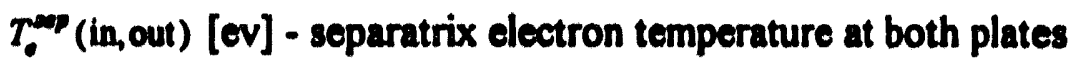
$T_{i}^{\infty}$ (in, out) [ev] - separatrix ion temperature at both plates $q_{\text {in }}^{\text {et }}$ (in, out) $\left[W / \mathrm{cm}^{2}\right]$ - peak heat flux along each plates 
$\lambda_{e}^{\text {ned }}$ (in, out) $[\mathrm{cm}]$ - radial decay length of peak heat flux

$\int q_{100}$ (in, out) $[\mathrm{W}]$ - total energy taken into the sheath by the plasma along each plate $\int \Gamma_{l}$ (in, out) $\left[s^{-1}\right]$ - integrated particle flux to each plate

Table 20: Comparison of Recycling Models for Simulation 1

\begin{tabular}{|c|c|c|c|}
\hline Quantity & b2/DEGAS results & b2 molec. physics & b2 enhanced \\
\hline$S_{n}^{\mu k}$ (in, out) $\left[\mathrm{cm}^{-3}\right]$ & $5.2 \mathrm{e} 18,8.9 \mathrm{e} 18$ & $4.5 e 18,8.8 e 18$ & $3.7 \mathrm{e} 18,8.8 \mathrm{e} 18$ \\
\hline$\lambda_{s_{\mathrm{n}}}^{\text {nd }}$ (in, out) $[\mathrm{cm}]$ & $2.0,3.4$ & $2.7,3.3$ & $2.0,3.4$ \\
\hline$\lambda_{s_{n}}^{p_{1}^{\prime}}$ (in, out) $[\mathrm{cm}]$ & $1.4,0.49$ & $1.3,0.63$ & $1.4,0.49$ \\
\hline$S_{\alpha}^{\mu k}$ (in, out) $\left[W / \mathrm{cm}^{3}\right]$ & $-33,-42$ & $-27,-42$ & $-26,-42$ \\
\hline$\lambda_{s_{\infty}}^{r \prime \prime}$ (in, out) [cm] & $2.2,4.2$ & $2.9,3.8$ & $2.6,4.5$ \\
\hline$\lambda_{s_{m}}^{\text {pol }}$ (in, out) $[\mathrm{cm}]$ & $1.0,0.44$ & $1.0,0.55$ & $1.0,0.44$ \\
\hline $\int S_{n}$ (in, out) $\left[\mathrm{s}^{-1}\right]$ & $4.6 \mathrm{e} 22,4.9 \mathrm{e} 22$ & $3.8 \mathrm{e} 22,5.0 \mathrm{e} 22$ & $4.2 e 22,5.0 e 22$ \\
\hline $\int S_{\infty}$ (in, out) $[\mathrm{W}]$ & $-2.6 e 5,-2.5 e 5$ & $-2.1 \mathrm{es},-2.4 \mathrm{e} 5$ & $-2.4 e 5,-2.5 e 5$ \\
\hline $\int S_{d}$ (in, out) $[\mathrm{W}]$ & $1.0 \mathrm{e} 4,3.1 \mathrm{e} 4$ & $4.4 \mathrm{e}, 3.4 \mathrm{e} 4$ & $9.1 e 3,3.1 e 4$ \\
\hline$n_{0}^{\infty \prime \prime}$ (in, out) $\left[\mathrm{cm}^{-3}\right]$ & $1.2 \mathrm{e} 14,5.9 \mathrm{e} 13$ & $8.7 e 13,6.2 e 13$ & $9.0 \mathrm{e} 13,6.2 \mathrm{e} 13$ \\
\hline$T_{0}^{\infty}$ (in, out) [ev] & $2.7,17$ & $5.7,15$ & $4.8,15$ \\
\hline$T_{i}^{\text {nop }}$ (in, out) [ev] & $4.2,2.4$ & $4.3,2.7$ & $4.6,2.7$ \\
\hline$q_{\text {tion }}^{\mathrm{k}}(\mathrm{in}$, out $)\left[\mathrm{W} / \mathrm{cm}^{2}\right]$ & 43,90 & 50,82 & 44,82 \\
\hline$\lambda_{\mathrm{L}}^{\mathrm{mat}}$ (in, out) [cm] & $4.1,4.7$ & $6.2,7.0$ & $4.4,6.0$ \\
\hline $\int q_{\text {not }}$ (in, out) $[\mathrm{W}]$ & $1.9 \mathrm{es}, 6.6 \mathrm{es}$ & $2.4 e 5,6.1 e 5$ & $2.2 e 5,6.1 e 5$ \\
\hline $\int \Gamma_{1}$ (in, out) $\left[\mathrm{s}^{-1}\right]$ & $4.6 \mathrm{e} 22,5.2 \mathrm{e} 22$ & $4.0 \mathrm{e} 22,5.2 e 22$ & $4.0 \mathrm{e} 22,5.2 \mathrm{e} 22$ \\
\hline
\end{tabular}


Table 21: Comparison of Recycline Models for Simulation 2

Quantity

$S_{n}^{\mu k}$ (in, out) $\left[\mathrm{cm}^{-3}\right]$

$\lambda_{s_{\mathrm{s}}}^{\text {(in }}$ (in out) $[\mathrm{cm}]$

$\lambda_{s_{1}}^{p d}$ (in, out) [cm]

$S_{\omega}^{\mu k}$ (in, out) $\left[W / \mathrm{cm}^{3}\right]$

$\lambda_{s_{m}}$ (in, out) [cm]

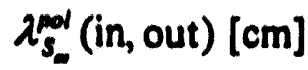

$\int S_{n}$ (in, out) [s-1]

$\int S_{\infty}$ (in, out) [W]

$\int S_{d}$ (in, out) [W]

$n_{e}^{\text {esp }}$ (in, out) $\left[\mathrm{cm}^{-3}\right]$

$T_{0}^{\text {ent }}$ (in, out) [ev]

$T_{i}^{\text {enp }}$ (in, out) [ev]

$q_{\text {tol }}^{\text {et (in, out) }\left[W / \mathrm{cm}^{2}\right]}$

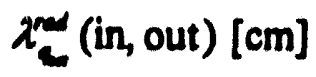

$\int q_{10 t}$ (in, out) [W]

$\int \Gamma_{1}($ in, out $)\left[s^{-1}\right]$
b2/DEGAS results

$7.4 \mathrm{e} 17,2.3 \mathrm{e} 18$

$2.2,2.4$

$* *, 0.73$

7.0, 14

$2.2,2.6$

$* *, 0.61$

$1.8 \mathrm{e} 22,1.7 \mathrm{e22}$

$-1.105,-8.9 e 4$

$-6.9 e 3,2.7 e 3$

$5.2 e 13,3.2 e 13$

$1.8,12.5$

$4.5,3.8$

12,34

$2.9,4.6$

$1.105,2.5 e 5$

$1.7 e 22,1.9 e 22$ b2 molec. physics

$7.2 \mathrm{e} 17,1.9 \mathrm{e} 18$

$3.7,2.5$

$* *, 1.5$

$3.5,14$

$3.7,2.7$

$* *, 1.0$

$1.5 e 22,1.9 e 22$

-1.0 es, - -1.0es

$-8.4 e 3,4.4 e 3$

$4.8 \mathrm{e} 13,4.7 \mathrm{e} 13$

$3.0,7.4$

$5.2,4.8$

17,36

$5.9,6.2$

$8.904,2.305$

$1.5 e 22,2.1 e 22$ b2 enhanced rad.

$6.0 e 17,2.4 e 18$

$3.7,2.7$

$* *, 2.6$

$3.2,16$

$3.5,2.9$

**, 1.0

$1.5 e 22,2.0022$

$-9.004,-1.105$

$-6.7 e 3,3.2 \bullet 3$

$5.0013,4.4 e 13$

$2.6,8.6$

5.04 .5

16,32

$5.7,6.4$

$7.9 e 4,2.5 e 5$

$1.5 e 22,2.0 e 22$

** Source peak several cells poloidally off of the plate 
Table 22: Comparison of Recycling Models for Simulation 3

\section{Quantity}

$S_{n}^{p k}$ (in, out) $\left[\mathrm{cm}^{-3}\right]$

$\lambda_{s_{n}}$ (in, out) [cm]

$\lambda_{s_{e}}$ (in, out) [cm]

$S_{\infty}^{m k}$ (in, out) $\left[W / \mathrm{cm}^{3}\right]$

$\lambda \bar{s}_{m}^{m}$ (in, out) [cm]

$\lambda s_{s_{m}}$ (in, out) [cm]

$\int S_{n}$ (in, out) [8-1]

$\int S_{\infty}$ (in, out) [W]

$\int S_{d}$ (in, out) [W]

$n_{0}^{\text {ent (in, out) }}\left[\mathrm{cm}^{-3}\right]$

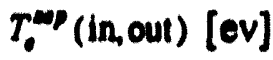

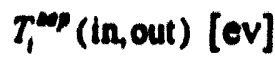

$q_{\text {int }}^{\text {(in, out) }\left[W / \mathrm{cm}^{2}\right]}$

$\lambda^{m a d}$ (in, out) [cm]

$\int g_{m \text { (in }}$ (int) [W]

$\int \Gamma_{1}$ (in, out) $\left[s^{-1}\right]$
b2/DEGAS results

$7.5 e 18,9.4 e 18$

$2.5,3.1$

$1.1,0.76$

24,38

$2.5,3.9$

$0.91,0.51$

$5.7 e 22,4.7 e 22$

$-2.6 e 5,-1.8 e 5$

$1.304,2.904$

$1.3 e 14,7.3 e 13$

$1.9,13$

$3.8,2.7$

26,82

$8.6,5.6$

$1.8 \mathrm{es}, 6.6 \mathrm{es}$

$4.8 e 22,5.6 e 22$ b2 molec. physics

$4.6,9.1 \mathrm{e} 18$

$2.8,3.2$

1.0, 0.69

19,45

$3.5,3.7$

$0.80,0.59$

$4.3 e 22,5.6 e 22$

$-2.3 e 5,-2.7 e 5$

$-2.9 e 3,3.4 e 4$

$1.0 \mathrm{e} 14,7.6 \mathrm{e} 13$

$3.3,11.6$

4.6, 3.4

36,72

$6.7,7.4$

$1.9 \mathrm{es}, 6.0 \mathrm{es}$

$4.3 e 22,5.6 e 22$ b2 enhanced rad.

$3.9,9.0 e 18$

$4.3,3.2$

$0.7,0.67$

19,45

4.5, 3.7

$0.70,0.57$

4.7e22, $5.5 e 22$

$-2.9 e 5,-2.7 e 5$

$-2.104,3.604$

$1.3 e 14,7.5 e 13$

$1.7,11.8$

$3.7,3.3$

25,72

$8.1,7.2$

1.5es, 5.6es

$4.6 e 22,5.6 e 22$ 
Table 23: Comparison of Recycling Models for Simulation 4

Quantity

$S_{n}^{\mu k}$ (in, out) $\left[\mathrm{cm}^{-3}\right]$

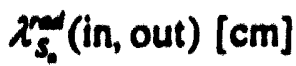

$\lambda_{s_{0}}$ (in, out) $[\mathrm{cm}]$

$S_{\omega}^{\mu k}($ in, out $)\left[W / \mathrm{cm}^{3}\right]$

$\lambda_{s_{m}}$ (in, out) [cm]

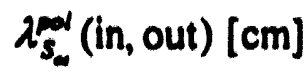

$\int S_{n}$ (in, out) [ $\left.\mathbf{s}^{-1}\right]$

$\int S_{\infty}$ (in, out) [W]

$\int S_{d}$ (in, out) [W]

$n_{0}^{n+1}$ (in, out) [ $\left.\mathrm{cm}^{-3}\right]$

$T_{e}^{\text {enp }}$ (in, out) [ov]

$T_{1}^{\text {enp }}$ (in, out) [ov]

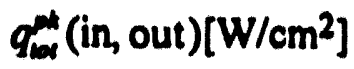

$\lambda^{m}$ (in, out) [cm]

$\int g_{\text {out }}$ (in, out) [W]

$\int \Gamma_{1}$ (in, out) [s-1]
b2/DEGAS results

$2.6 e 18,3.6 e 18$

$4.4,5.6$

$0.73,0.43$

12,17

$5.5,6.7$

$0.73,0.44$

$1.8 \mathrm{e} 22,2.5 e 22$

$-8.904,-1.205$

$9.6 e 3,8.1 e 3$

$1.9 \mathrm{e} 13,1.7 \mathrm{el}$

$3.6,4.7$

$2.9,4.2$

110,109

$4.7,6.6$

4.7es, 8.7es

$2.2 e 22,3.2 e 22$ b2 molec. physics b2 enhanced rad.

$2.8 \mathrm{e} 18,3.7 e 18$

$3.3,3.9$

$1.0,0.57$

12, 17

$3.9,4.3$

$0.99,0.56$

$1.8 \mathrm{e} 22,2.5 \mathrm{e} 22$

$-9.3 e 4,-1.2 \mathrm{es}$

$9.9 e 3,1.804$

$2.1 \mathrm{e} 13,1.8 \mathrm{e} 13$

$3.3,4.4$

$2.3,2.0$

100,105

$5.2,7.2$

4.4eS, 8.4e5

$2.3 e 22,3.2 e 22$
$2.6 e 18,3.7 e 18$

$3.3,3.9$

$1.0,0.56$

13,17

$3.9,4.4$

$1.00,0.56$

$1.7 e 22,2.5 e 22$

$-9.9 e 4,-1.2 e 5$

$8.0 e 3,1.604$

$1.9 \mathrm{e} 13,1.7 \mathrm{e} 13$

$3.5,4.5$

$2.4,2.2$

99, 105

$5.2,7.2$

$4.4 \mathrm{es}, 8.4 \mathrm{es}$

2.2e22. 3.1e22 
Table 24: Comparison of Recycline Models for Simulations

Quantity

$S_{n}^{\text {pt }}$ (in, out) $\left[\mathrm{cm}^{-3}\right]$

$\lambda_{s_{\mathrm{p}}}^{\text {nd }}$ (in, out) [cm]

$\lambda_{s_{\mathrm{s}}}$ (in, out) [cm]

$S_{\omega}^{\mu k}$ (in, out) $\left[W / \mathrm{cm}^{3}\right]$

$\lambda_{S_{m}}^{n \pi m}$ (in, out) [cm]

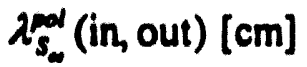

$\int S_{n}$ (in, out) $\left[s^{-1}\right]$

$\int S_{\infty}$ (in, out) $[W]$

$\int S_{\alpha}$ (in, out) [W]

nop (in, out) [cm-3]

$T_{0}^{\text {enp }}$ (in, out) [ev]

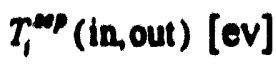

$q_{\text {of }}^{\text {kt (in, out) }}\left[\mathrm{W} / \mathrm{cm}^{2}\right]$

$\lambda^{m}$ (in, out) [cm]

$\int g_{m x}($ in, out) $[\mathrm{W}]$

$\int \Gamma_{1}$ (in, out) [s-1]
b2/DEGAS results

$1.9 \mathrm{e} 19,1.8 \mathrm{e} 19$

5.0, 10.1

$0.56,0.36$

77,81

$6.5,11.2$

$0.53,0.33$

$9.3 e 22,9.3 e 22$

$-3.9 e 5,-4.0 e 5$

$1.6 \mathrm{es}, 1.4 \mathrm{es}$

$5.1 e 13,3.5 e 13$

97, 137

$<1,<1$

1060,960

$3.0,4.3$

$3.206,5.5 e 6$

$9.0622,1.0623$ b2 molec. physics

$$
2.0019,2.0019
$$

$3.9,9.0$

$0.54,0.34$

80,84

$5.0,11.7$

$$
0.53,0.33
$$

$8.7 e 22,1.0 .23$

$-3.7 e 5,-4.3 \mathrm{es}$

$1.4 \mathrm{es}, 1.5 \mathrm{es}$

$5.2 e 13,3.5 e 13$

96,130

$<1,<1$

1080,1010

$2.9,3.8$

$3.2 e 6,5.406$

$9.0022,1.0 \times 23$ b2 enhanced rad.

2.1e19, 2.1e19

$3.7,9.0$

$0.54,0.34$

90,84

$4.8,11.6$

$0.53,0.33$

$8.8 e 22,1.0 e 23$

$-4.105,-4.3 \mathrm{es}$

$1.3 \mathrm{es}, 1.5 \mathrm{es}$

$5.2013,3.4 \mathrm{e} 13$

95, 130

$<1,<1$

1080,1000

$2.8,3.8$

$3.106,5.406$

$9.0022,1.0 \times 23$ 
Trble 25. Comprison of Regycline Modele for Simulntion 6

Quantity

$S_{n}^{\mu}$ (in, out) $\left[\mathrm{cm}^{-3}\right]$

$\lambda_{1}^{m}$ (in, out) [cm]

$\lambda s_{s}^{-1}($ in, out $)[\mathrm{cm}]$

$S_{\infty}^{m}$ (in, out) [W/cm $]$

$\lambda_{s}$ (in, out) [cm]

$\lambda_{s_{\alpha}}($ in, out) $[\mathrm{cm}]$

$\int S_{n}$ (in, out) $\left[s^{-1}\right]$

$\int S_{\omega}$ (in, out) [W]

$\int S_{\alpha}$ (in, out) [W]

ne"s (in, out) [cm-3]

$T_{1}^{\text {esp }}$ (in, out) [ov]

$T_{1}^{e \prime \prime}$ (in, out) [ov]

$q_{\text {min }}^{\text {(in, out) }}\left[\mathrm{W} / \mathrm{cm}^{2}\right]$

$\lambda_{\mathrm{c}}^{\text {nde }}$ (in, out) [cm]

$\int q_{m}$ (in, out) [W]

$\int \Gamma_{1}$ (in, out) $\left[s^{-1}\right]$
b2/DEGAS results

$6.5 e 18,8.5 e 18$

$3.0,3.6$

$0.66,0.60$

32,37

$3.0,4.2$

$0.66,0.60$

$2.8 e 22,3.5 e 22$

$-1.365,-1.6 e 5$

$3.304,1.404$

$2.3 e 13,2.0613$

93, 110

$<1,<1$ ov

460,410

$2.5,3.0$

9.9e5, 1.806

$2.8022,3.7022$ b2 molec. physics

$1.3 e 19,1.3619$

$2.0,2.5$

$2.2,2.7$

51,55

2.2, 2.7

$0.64,0.44$

$3.1022,4.2 e 22$

$-1.405,-1.965$

$2.804,4.204$

$4.3013,3.1013$

66,90

$<1,<1$ ov

440,470

2.6, 3.1

$9.465,1.806$

$3.2022,4.4022$ b2 enhanced rad.

$1.3019,1.3 e 19$

$2.0,2.5$

$2.2,2.7$

57,55

$2.2,2.7$

$0.65,0.46$

$3.1 \in 22,4.2622$

$-1.505,-1.905$

$2.804,4.204$

$4.4013,3.1013$

58,90

$<1,<1$ ov

440,470

$2.7,3.1$

$9.2 e 5,1.806$

$3.2 e 22,4.4022$ 
Tnble 26. Comprision of Recycline Modele for Simulntion 7

Quantity

$S_{n}^{\text {nt }}(\mathrm{in}$, out $)\left[\mathrm{cm}^{-3}\right]$

$X_{\mathrm{f}}^{4}$ (in, out) [cm]

$\lambda_{\mathrm{s}}^{\mathrm{m}}(\mathrm{in}$, out $)[\mathrm{cm}]$

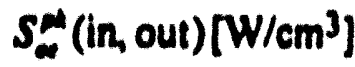

$\lambda_{s_{-}}$(in, out) [cm]

$\lambda s_{s}^{*}$ (in, out) [cm]

$\int S_{n}$ (in, out) $\left[s^{-1}\right]$

$\int S_{\infty}$ (in, out) [W]

$\int S_{\alpha}($ in, out $)[W]$

$n_{0}^{(0)}\left(\right.$ In, out) $\left[\mathrm{cm}^{-3}\right]$

$T_{0}^{\text {and }}$ (in, out) [ov]

$T_{1}^{\infty 10}($ in, out) [ev]

$q_{\text {(in }}^{\text {(in, out })\left[W / \mathrm{cm}^{2}\right]}$

$x_{\mathrm{c}}$ (in, out) $[\mathrm{cm}]$

$\int q_{m}$ (in, out) [W]

$\int \Gamma_{1}($ in, out $)\left[s^{-1}\right]$
b2/DEGAS results

$4.2619,3.4619$

$2.0,2.1$

$0.48,0.37$

$.250, \cdot 140$

$1.9,3.8$

$0.45,0.34$

$1.8023,1.6023$

$-7.205,-6.765$

$2.3 \mathrm{eS}, 1.8 \mathrm{es}$

$7.2813,3.7 \bullet 13$

82, 129

$<1,<1$

1200,1010

2.5, 3.2

$3.306,6.106$

$1.9023,1.7023$ b2 molec. physic:

$3.2 \times 19,4.2619$

$1.3,1.0$

$0.53,0.30$

$.120,-170$

$1.9,1.6$

$0.53,0.30$

$1.4023,1.8023$

$-6.005,-7.305$

$1.605,2.3 e 5$

$6.7 e 13,5.0613$

86, 116

$<1,<1$

1210,1140

2.6, 3.2

$3.506,0.206$

$1.5023,1.8023$ b2 enhanced rad.

$3.9619,4.7619$

$0.9,0.9$

$0.54,0.30$

$.170,-190$

$1.2,1.4$

$0.53,0.29$

$1.6 e 23,1.9623$

$-7.105,-7.7 \mathrm{es}$

$1.504,2.404$

$7.4013,5.6013$

80,109

$4.6,2.7$

1190,1160

2.5. 3.1

$3.306,6.206$

$1.6023,1.9623$ 
Trble 27. Comonrison of Regyaline Modele for Simulution

\begin{tabular}{|c|c|c|}
\hline Quantity & b2/DEOAS resulte & b2 molec physics \\
\hline$S_{n}^{*}($ In, out $)\left[\mathrm{cm}^{-3}\right]$ & $1.0025,2.5 e 25$ & $1.9025,1.9025$ \\
\hline$\lambda_{4}^{2}(\mathrm{in}$, out $)[\mathrm{cm}]$ & $7.5,5.9$ & $3.8,4.4$ \\
\hline$\lambda_{\mathrm{A}_{1}}^{\prime}(\mathrm{in}, \mathrm{out})[\mathrm{cm}]$ & $0.42,0.44$ & $0.60,0.44$ \\
\hline$s_{\infty}^{m}(i n, o u t)\left[W / \mathrm{cm}^{3}\right]$ & $-4.387,-1.008$ & $-7.307,-7.507$ \\
\hline$x_{\mathrm{f}_{m}}(\mathrm{in}, \mathrm{Out})[\mathrm{cm}]$ & $8.5,9.7$ & $4.9,3.2$ \\
\hline$\lambda s_{s_{0}}^{\prime \prime}($ in, out $)[\mathrm{cm}]$ & $0.41,0.44$ & $0.61,0.45$ \\
\hline $\int S_{n}($ in, out $)\left[s^{-1}\right]$ & $5.6 e 22,1.7023$ & $8.6 \mathrm{e22}, 1.4023$ \\
\hline $\int S_{\omega}($ in, out $)[W]$ & $-2.585,-7.105$ & $-3.605,-5.8 \mathrm{es}$ \\
\hline $\int s_{\alpha}($ in, out $)[W]$ & $6.404,2.305$ & $1.405,2.105$ \\
\hline$n_{0}^{n+1}($ in, our $)\left[\mathrm{cm}^{-3}\right]$ & $3.0013,6.2013$ & $5.2013,4.6013$ \\
\hline$T_{0}^{\infty}(\ln$, out $)[\mathrm{ov}]$ & 124,87 & 94,107 \\
\hline$T_{1}^{\infty}($ in, out $)[o v]$ & $<1<1<1$ ov & $<1<1$ ov \\
\hline$q^{*}(\mathrm{in}$, out $)\left[W / \mathrm{cm}^{2}\right]$ & 970,820 & 1060,930 \\
\hline$x_{\mathrm{c}}^{\prime \prime}(\mathrm{in}, \mathrm{out})[\mathrm{cm}]$ & $3.4,3.1$ & $2.8,4.5$ \\
\hline $\int q_{m}(i n$, out $)[W]$ & $3.006,5.606$ & $3.006,5.006$ \\
\hline $\int \Gamma_{1}($ in our $)\left[n^{-1}\right]$ & $6.3022,1.7023$ & $8.9022,1.4023$ \\
\hline
\end{tabular}


Tnble 28: Compndion of Regyaline Modele for Simulutions

Quantity

$S_{n}^{m}($ in, out $)\left[\mathrm{cm}^{-3}\right]$

$x_{4}^{2}($ in, out) $[\mathrm{cm}]$

$\lambda q_{1}^{\prime}($ in, out $)[\mathrm{cm}]$

$S_{\omega}^{m}($ in, out $)\left[W / \mathrm{cm}^{3}\right]$

$x_{s}^{*}($ in, out) $[\mathrm{cm}]$

$\lambda s_{*}^{\prime \prime}($ in, out) $[\mathrm{cm}]$

$\int S_{n}($ in, out $)\left[v^{-1}\right]$

$\int S_{\omega}($ in, out $)[W]$

$\int S_{d}($ in, out) (W)

$n$ "e' (In, out) [em-3]

$T_{0}^{m}($ In, ow $)[0 v]$

$T_{1}^{\text {an) (In, oul) [ov] }}$

Selt (in, out) [W/ $\left./ \mathrm{cm}^{2}\right]$

$x$ (in, out) [cm]

$\int q_{m}$ (in, out) $[W]$

$\int r_{1}($ in, out $)\left[r^{-1}\right]$
b2/DEOAS results

$1.8019,3.2019$

$3.1,7.1$

$0.96,0.35$

74,140

$6.7,8.2$

$0.53,0.32$

$7.5 \times 22,1.1023$

$-3.305,-4.7 e 5$

$1.265,1.805$

$4.8013,5.5013$

101, 105

$2.6,4.6$

1190,1160

$2.8,3.7$

$3.206,5.906$

7.7022, 1.1023 b2 molec physics

$2.9019,2.8019$

$3.9,4.9$

$0.52,0.28$

107,110

$4.2,9.7$

$0.52,0.28$

$7.6 e 22,1.1023$

$-3.305,-4.705$

$1.205,1.705$

$7.0613,5.6013$

92,87

14.0, 1,9

1530, 940

$2.8,3.4$

$3.506,5.606$

$8.0022,1.0023$ b2 enhanced rad.

$3.1019,2.8619$

$3.5,4.9$

$0.53,0.28$

130, 100

4.3, 5.6

$$
0.53,0.28
$$

$7.6022,1.1023$

$-3.605,-4.705$

$1.105,1.705$

$7.5013,5.5013$

86,87

$10,2.0$

1900,940

$3.8,3.4$

$3.506,5.606$

$8.1022,1.0023$ 


\section{REFERENCES}

[1] B. J. Braums. "Computational Studies in Tokamak Equilibrium and Transport". Ph.D. theais, Univeraity of Utrecht, The Netherlands, 1986.

[2] D. B. Heifiza, et. al. "A Monte-Carlo Model of Neutra-Plama Transport in Diverted Plaumas", J. Comp. Phy. 16 (1982), 309.

[3] D. P. Stotler, et. al. "Compact Ignition Tokamak Edge and Divertor Modeling, Bull. Am. Phy. Soc. 38, no. 9 (1990) 2086.

[4] D. Conter, at. al. "Edge Plasma Modeling of TFTR with the b2 2-D Fluld Code", Bull. Am. Phy. Soc. 35, no. 9 (1990) 1921.

[5] M. A. Rensink. "Summary on Grid Generation", presented at the ITERBPX Edge Modeling Workahop, hald at Lawrence Livermore National Laboratory, Jan. 14-16, 1992.

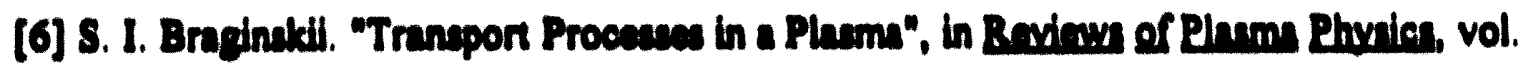
1. (M. A. Leontovich, Bd.), Conmultants Bureau, Now York, 1965;pp. 205-311.

[7] B. J. Brams. "A Multi-Fluid Code for Simulation of the Edge Plaems in Tokamaks", NET Report EUR-FU/XII-80/87/68, 1987. 
[8] B. J. Braams and C. E. Singer. "Low Temperature Plasma near a Tokamak Reactor Limiter", Fus. Tech. 9 (1986) 320.

[9] K. A. Werloy, "2-D MHD Calculations of Plasma-Particle Flow with Pumping", presented at the Workshop on Power and Particle Control for ITER, held at Princeton Plauma Physics Laboratory, Jan. 9.10, 1990.

[10] K. A. Werley. "SOL Parameter Variation for ITER: D, Viscosity, Recyeling, and Impurity Radiation in the b2 Code", presented at the Workshop on Edge Plasma Phyaics for BPX and ITER, held at Princeton Plasma Physics Laboratory, Jan. 15. $17,1991$.

[II] K. A. Werley. "ITER CDA and HARD Modeling", presented at the ITERBPX Edge Modeling Workehop, held at Lawrence Livermore National Laboratory, Jan. 14-16, 1992.

[12] J. Neuhauser, et. a. "Tokamak Edge Modeling and Comparison with Experiment in ASDEX", Plas. Phy. Cont. Fus. 31, no.10 (1989) 1551.

[13] M. A. Renaink, a. a. "Fluid Model Simulation of the Scrape-off Layer in DIII-D Single-null Divenor Plaemas, Bull. Am. Phy. Soc. 3S, no. 9 (1990) 2024.

[14] M. A. Renaink, A. d. "Modaling of DIII-D Divertor Plamas at ITER-like Power Lovals", Bull. Am. Phy. Soc. 36, no. 9 (1991) 2472. 
[15] A. Prinja and D. A. Knoll. "2-D Edge Plasma Simulations of an H-mode in JET", presented at the Workshop on Power and Particle Control for ITER held at Princeton Plasma Physics Laboratory, Jan. 9-10, 1990.

[16] M. Sugihara, et. al. "Numerical Analyses of Plasma and Neutral Particle Behavior and Design Criteria for Poloidal Divertor in Fusion Experimental Reactor", J. Nuc. Mar. $128 \& 129(1984) 114$.

[17] Y. L. Igitkhanov, ot. al. "Advanced Two-Dimensional Simulation of Edge Plasma in a Poloidal Divertor", Plas. Phy. Cont. Nuc. Fus. Res. (Proc. 10th Int. Conf. London, IAEA, 1984), vol.2 (1985) 113.

[18] N. Ueda, et. al. "Development of a Two-Dimensional Fluid Code and Its Application to the Doublet III Divertor Experiment", Nuc. Fus. 28, no. 7 (1988) 1183.

[19] N. Ueda, K. Itoh, and S. I. Itoh. "Two-Dimensional Analysis of Limiter/Divertor Transition in Scrape-off Layer Plasmu", Nuc. Fus. 29, no. 2 (1989) 173.

[20] M. Petravic. "Transport Coefticients in the DIn-D SOL", presented at the Workehop on Power and Particle Control for ITER, held at Princeton Plasma Physics Laboratory, Jan. 9-10, 1990.

[21] E. L. Vold, et. al. "Tokamak Plasma Edge Comparicons: Comparicons to Experiment and Between Two 2-D Codes", presented at the Workshop on Power and Particle Control for ITER, hald at Princeton Plauma Phyaics Laboratory, Jan. 9-10, 1990. 
[22] M. Day, et. al. "Plasma/Neutral Fluid Simulations on a Non-orthogonal Finite Volume Mesh", Bull. Am. Phy. Soc. 36, no. 9 (1991) 2434.

[23] D. A. Knoll. "An Advanced Newton Solution Algorithm for the Tokamak Edge Plasma Fluid Equations", Bull. Am. Phy. Soc. 36, no. 9 (1991) 2489.

[24] T. D. Rognlien. "Status of the LEDGE code", presented at the ITER/BPX Edge Modeling Workshop, held at Lawrence Livermore National Laboratory, Jan. 14-16, 1992.

[25] R. B. Campbell, et. al. "A Calculation of Impurity Transport in the DIII-D Scrapeoff Layer", Bull. Am. Phy. Soc. 36, no. 9 (1991) 2471.

[26] D. P. Stotler. Private communication, 1991.

[27] S. A. Khan and T. D. Rognlien. "Thermal Heat Flux in a Plasma for Arbitrary Collisionality", Phys. Fluids 24, vol. 8 (1981) 1442.

[28] T. D. Rognlien. "Finite Mean-Free-Path Corrections to Parallel Heat Flow in Divertors", presented at the US ITER Workshop on Impurity Transport, held at Princeton Plasma Physics Laboratory, May 23-26, 1989.

[29] P. C. Stangeby. "The Plasma Sheath", in Physics of Plasma-Wall Interactions in Controlled Eusion (D. E. Post and R. Behrisch, Ed.). Plenum Press, New York (1986) 58.

[30] K. A. Werley, private communication. 
[31] J. T. Hogan. "Edge Modeling in the ORNL Edge Plasma and Particle Control Program", presented at the ITER/BPX Edge Modeling Workshop, held at Lawrence Livermore National Laboratory, Jan. 14-16, 1992.

[32] D. B. Heifitz. "Neutral Particle Transport", in Physics of Plasma-Wall Interactions in Controlled Eusion (D. E. Post and R. Behrisch, Ed.). Plenum Press, New York (1986) pp.695-771.

[33] R. K. Janev, et. al. J. Nuc. Mat. 121 (1984) 10.

[34] J. T. Hogan, private communication, 1992.

[35] P. C. Stangeby and D. M. McCracken. "Plasma Boundary Phenomena in Tokamaks", Nuc. Fus. 30, no. 7 (1990) 1225-1379.

[36] M. Rensink, private communication, 1992.

[37] C. M. Greenfield, et. al. "Transport Analysis of VH-mode Discharges in DIII-D", Bull. Am. Phy. Soc. 36, no. 9 (1991) 2475.

[38] L. Lao, et. al. Nuc. Fus. 25 (1985) 1611.

[39] L. Spitzer and R. Harm. Phys. Rev. 89 (1953) 977.

[40] G. F. Matthews, et. al. "The Sheath Power Transmission Factor in DIII-D", to be submitted to Nuc. Fus. 
[41] A. H. Futch, et.al. "Spatial Dependence of the Sheath Power Transmission Factor in DIII-D", DIII-D Physics Memo no. 9103, Feb. 1991.

[42] D. Guilhem. "Ergodic Divertor Studies on Heat and Particle Flux in Tore Supra", Bull. Am. Phy. Soc. 35, no. 9 (1990) 1998.

[43] T. Uckan, et. al. "Modular Pump Limiter Systems for Large Tokamaks", Fus. Tech. 13 (1988) 165.

[44] T. D. Rognlien, J. L. Milovich, and M. E. Rensink. "Divertor Plate Biasing, CrossField Drifts, and an Implicit Method with the b2 Code", presented at the Workshop on Edge Plasma Physics for BPX and ITER, held at Princeton Plasma Physics Laboratory, Jan. 15-17, 1991.

[45] L. Owen, private communication, 1991.

[46] C. C. Klepper, et. al. "Particle Exhaust Studies in Tore Supra with a Pump Limiter", Proc. 9th Int. Conf. on Plas. Surf. Int. in Cont. Fus. Dev., Bournemouth (1990).

[47] C. C. Klepper, et. al. "Consideration of the Magnetic Field Ripple in the Design of Plasma Edge Components for Tore Supra", Fus. Tech. 14 (1988) 288.

[48] D. M. Goebel, et.al. "Langmuir Probe Measurements in the TEXTOR Tokamak During ALT-I Pump Limiter Experiments", Plas. Phy. Cont. Fus. 29, no.4 (1984) 479. 
[49] The ASDEX team. "The H-mode of ASDEX", Nuc. Fus. 29 (1989) 1959.

[50] D. Bohm. The Characterisitics of Electrical Discharges in Magnetic Fields (A. Guthrie and R. K. Wakerling, Eds.), McGraw Hill, New York, chap. 3 (1949).

[51] D. E. Post, R. Behrisch, and B. Stansfield. "Introduction to the Physics of Plasma Wall Interactions in Controlled Fusion", in Physics of Plasma-Wall Interactions in Controlled Eusion (D. E. Post and R. Behrisch, Ed.). Plenum Press, New York (1986) pp.1-39.

[52] P. K. Mioduszewskj. "Advanced Limiters", in Physics of Plasma-Wall Interactions in Controlled Eusion (D. E. Post and R. Behrisch, Ed.). Plenum Press, New York (1986) pp.891-929.

[53] F. Wagner and K. Lackner. "Divertor Tokamak Experiments", in Bhysics of PlasmaWall Interactions in Controlled Eusion (D. E. Post and R. Behrisch, Ed.). Plenum Press, New York (1986) pp.931-1003.

[54] D. E. Post and K. Lackner. "Plasma Models for Impurity Control Experiments", in Physics of Plasma-Wall Interactions in Controlled Eusion (D. E. Post and R. Behrisch, Ed.). Plenum Press, New York (1986) pp.627-693.

[55] M. A. Mahdavi, et. al. J. Nuc. Mat. 121 (1984) 184. 


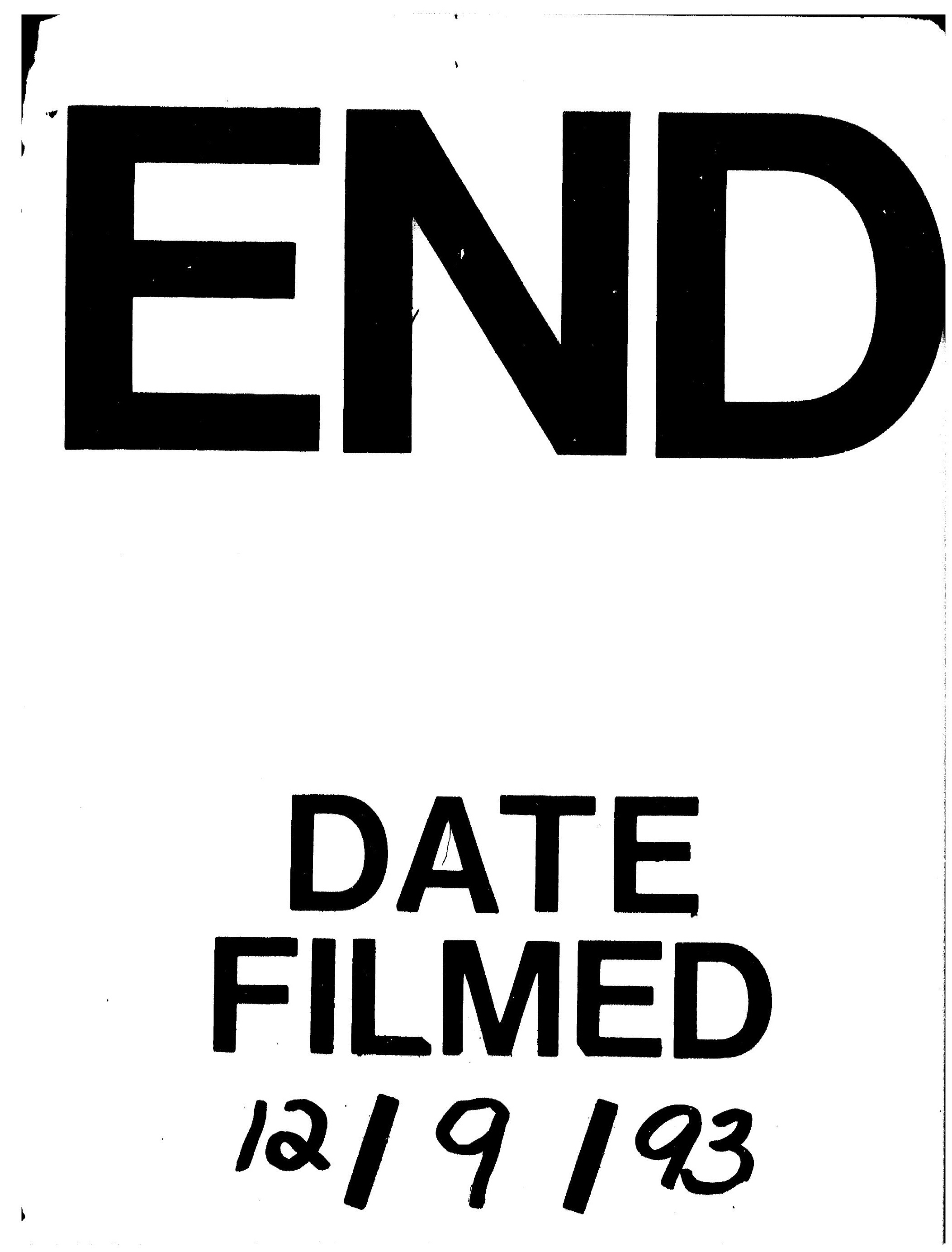


